\title{
PROPRIEDADES DE \\ CONJUNTOS-IGUALDADE DE CODIGOS
}

NAMI KOBAYASHI

DISSERTAÇÃO APRESENTADA

A0

INSTITUTO DE MATEMÃTICA E ESTATISTICA

DA

UNIVERSIDADE DE SÃO PAULO

PARA OBTENÇÃO DO GRAU DE MESTRE

EM

MATEMÁTICA APLICADA

AREA DE CONCENTRAÇĀO: CIENCIA DE COMPUTAÇÃO

ORIENTADOR: PROF. DR. IMRE SIMON

- SÃO PAUlo, ABRil de 1983 - 
Aos meus pais 


\section{AGRADECIMENTOS}

o meu muito obrigada

ao Professor Doutor Imre Simon,

pela excelente orientação, pelas crîticas e sugestões construtivas, pela colaboração intensiva e valiosa em to das as fases da realização deste trabalho e tambēm pela amizade, conjiança e estimulo demonstrados durante todo esse tempo;

aos meus alunos, colegas e amigos, pelo carinho, apoio e incentivo constantes, que foram im prescindiveis durante a elaboração deste trabalho;

ao Conselho Nacional de Desenvolvimento Científico e Tecnolögico (CNPq), pelo auxilio financeiro;

às bibliotecārias do IME-USP, em especial à Edmari G. Teixeira, pela atenção, dedicação e colaboração na pesquisa bibliogräfica;

à Luzia do Carmo Namiki e Regina Helena da Silva, pelo paciente e esmerado trabalho de datilografia.

Nami Kobayashi 
$\underline{S U M R R I O}$

PREFACIO . . . . . . . . . . . . . . . i-v

CAP. 1 - MORFISMOS E CONJUNTOS-IGUALDADE DE MORFISMOS DE MONOIDES LIVRES . . . . . . . . . . . . . . . 1

1. Morfismos de monüides livres . . . . . . 2

2. O conjunto-igualdade (minimal) de morfismos de monöides livres.......... . 7

CAP. 2 - MÄQUINAS DE TURING ............ 17

1. Definição . . . . . . . . . . . . 18

2. Descrição do funcionamento . . . . . . . 19

3. Mäquina de Turing reversíve1 . . . . . 29

4. Simulação de uma máquina de Turing determinística por uma reversivel e normalizada . . 34

5. Acoplamento de uma mäquina de Turing reversivel e normalizada com a sua reversa. . . 57

CAP. 3 - CASOS RESTRITOS DO PROBLEMA DE CORRESPONDENCIA $\mathrm{DE}$ POST . . . . . . . . . . . . . 61

1. Introdução . . . . . . . . . . 62

2. Construção de morfismos associados a uma mâ quina de Turing semi-normalizada . . . . 65

3. Codificação de uma computação . . . . . . 76

4. A indecidibilidade do Problema de Correspon dência de Post para morfismos que são côdigos de atraso um . . . . . . . . . . 82

5. A indecidibilidade do Problema de Correspon dência de Post para códigos de atraso un per tencentes a PS $\cap S P$. . . . . . . . . . . . . 98 
6. A condição de outras restrições do Problema de Correspondência de Post.. . . . . . 107

CAP. 4 - REPRESENTAÇÃO DE LINGUAGENS RECURS IVAMENTE ENU MERĀVEIS. . . . . . . . . . . . . 109

1. Uma representação de linguagens recursivamen te enumeräveis atravês de côdigos de atraso um em sentidos opostos......... . 110

2. Uma representação de linguagens recursivanen te enumeräveis através de cödigos de atraso um em sentidos opostos e pertencentes a PS . 151

3. Outras representações de linguagens recursi vamente enumeräveis . . . . . . . 155

CAP. 5 - A REGULARIDADE DE CONJUNTOS-IGUALDADE DE MORFIS MOS . . . . . . . . . . . . . 163

1. Restrições sobre os morfismos para que seus conjuntos-igualdade sejam regulares. . . . 164

2. A não efetividade de alguns conjuntos-igua1. dade regulares . . . . . . . . . 172

3. Conjuntos-igualdade não regulares . . . . 173

4. Restrições sobre os morfismos que representam as linguagens recursivamente enumeräveis atravës de conjuntos-igualdade.. . . . . 175

REFERENCIAS BIBLIOGRÄFICAS . . . . . . . . . . 177

INDICE DE SIMBOLOS . . . . . . . . . . . 180

INDICE DE DEFINIÇÕES . . . . . . . . . . . . . 181 


\section{PREFÁCIO}

o conjunto-igualdade de dois morfismos $f$ e g de monöi des livres, $f, g: \Gamma^{*} \longrightarrow \Delta^{*}$, consiste de todas as palavras em $\Gamma^{*}$ que têm a mesma imagem por $f$ e por $g$. Ou seja,

$$
E q(f, g)=\left\{w \in \Gamma^{*} / w f=w g\right\} .
$$

Tais conjuntos têm tido importância relevante na solução de värios problemas da Teoria de Linguagens Formais.

Observamos inicialmente que um conjunto-igualdade de morfismos (a menos da palavra vazia) tambëm pode ser interpre tado como representando o conjunto de soluções de uma instância do Problema de Correspondência de Post, da seguinte forma.

Seja $\Gamma$ um alfabeto com $n$ simbolos, $\Gamma=\left\{\gamma_{1}, \gamma_{2}, \ldots, \gamma_{n}\right\}$ e sejam duas $n$-uplas de palavras não vazias $\left(x_{1}, x_{2}, \ldots, x_{n}\right)$ e $\left(y_{1}, y_{2}, \ldots, y_{n}\right)$ sobre algum alfabeto $\Delta$. Consideremos os morfís mos $f, g: r^{*} \longrightarrow \Delta^{*}$, definidos por:

$$
\gamma_{i} f=x_{i} \quad \text { e } \quad \gamma_{i} g=y_{i} \text {, para cada } i \quad, \quad 1 \leq i \leq n
$$

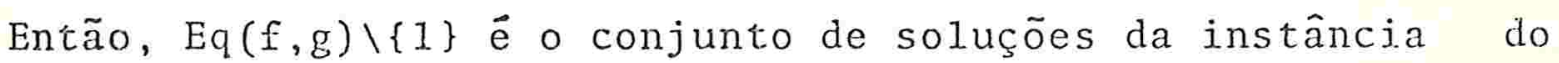
Problema de Correspondência de Post com as n-uplas $\left(x_{1}, x_{2}, \ldots, x_{n}\right)$ e $\left(y_{1}, y_{2}, \ldots, y_{n}\right)$. 
Como se sabe, o Problema de Correspondência de Post foi mostrado ser indecidível por E.L.Post (1946), ou seja, "Não existe um algoritmo para decidir se o conjunto-igualdade de dois morfismos dados contém ou não alguma palavra não va$z i a^{\prime \prime}$.

Mais tarde, N. Chomsky e M.P.Schlltzenberger (1963) mos traram que este problema permanece indecidive1, mesmo restrin gindo um dos morfismos para ser injetor.

Posteriormente, Y.Lecerf (1963a e b) provou a indeci dibilidade deste problema quando ambos os morfismos são injetores. Para isso, ele reduziu o Problema da Parada para o Pro blema de Correspondência de Post com morfismos injetores e,pa ra fazê-1o associou dois morfismos a uma mäquina de Turing e introduziu o conceito de mäquina de Turing reversiver.

Com o objetivo de escrever uma demonstração mais for mal dos resultados obtidos por Lecerf, adotamos um formalismo para descrever as mäquinas de Turing, inspirado no Iivro de S.Eizenberg (1974). Acreditamos que a utilização deste formalismo foi imprescindivel para a obtenção dos resultados mais importantes deste trabalho.

Como primeira consequência desta formalização, forta lecemos o resultado de Lecerf mostrando que:

"O ProbZema de Correspondência de Post permanece indecidivel, mesmo quando restrito a códigos de atraso um". 
Chamamos de código a um morfismo injetor.

Mais recentemente, a investigação intensa de Sistemas de Lindenmayer possibilitou o uso de conjuntos-igualdade na solução de vārios problemas de interesse nesta teoria. Por exemplo, na decidibizidade do Problema da Equivalência da Seqllência para os Sistemas DOL. Estes resultados motivaram um estudo mais detalhado sobre a classe dos conjuntos-igualdade de morfismos, como se pode ver em A.SaZomaa (1978), J.Enge Ifriet e G.Rozenberg (1979 e 1980) e G.Rozenberg e A.Salomax (1980, capítulo III).

Por outro 1ado, B.Courcelze (1978), J.Karhumaki e I.Simon (1979) e G.Rozenberg e A.SaZomaa (1980) tinham encontrado restrições sobre os morfismos para que seus conjuntos-igualdade fossem regulares. Embora as demonstrações efetuadas, via de regra, não fossem construtivas, não se sabia se era pos sivel construir algoritmicamente os conjuntos-igualdade em questão.

Então, como consequência da indecidibilidade do Problema de Correspondência de Post para cödigos de atraso um, mostramos que, em geral, os conjuntos-igualdade regulares men cionados acima não são efetivamente reguzares. Mais precisamen te, temos que:

\footnotetext{
"Se $f$ e g são cödigos de atraso um, então o conjunto-igualdade $E_{q}(f, g)$, apesar de ser regular, não ë efetivamente regular".
} 
Ao mesmo tempo, os conjuntos-igualdade de morfismos de monöides livres vinham sendo utilizados tambëm em represen tacões de linguagens recursivamente enumeráveis.

Por exemplo, A.Salomaa (1978) exibiu uma representação de linguagens recursivamente enumeráveis como imagens por uma função dgsm de conjuntos-igualdade de morfismos de monói des livres e, no ano seguinte, K. Culik (1979b) fortaleceu es se resultado restringindo a função dgsm para um morfismo e substituindo o conjunto-igualdade pelo seu conjunto-igualdade minima1, obtendo:

"Para cada linguagem recursivamente enumerävel $L \subseteq \Sigma^{*}$, existem efetivamente um morjismo $h_{\Sigma}: \Gamma^{*} \longrightarrow \Sigma^{*}$, dois morfismos $h_{I}$, $\mathrm{h}_{2}: \mathrm{r}^{*} \longrightarrow \Delta^{*}$ tais que $\mathrm{L}=\left(\mathrm{eq}\left(\mathrm{h}_{1}, \mathrm{~h}_{2}\right)\right) \mathrm{h}_{\Sigma} "$.

0 conjunto eq $\left(h_{1}, h_{2}\right)$ denota o conjunto-igualdade minimal de $h_{1}$ e $h_{2}$, isto $\vec{e}$,

$$
\begin{array}{r}
\text { eq }\left(h_{1}, h_{2}\right)=\left\{w \in \Gamma^{+} / w_{1}=w h_{2} \text { e se } w=u v \text { para u e } v \text { em } \Gamma^{+}\right. \\
\text {então } \left.u h_{1} \neq u h_{2}\right\} .
\end{array}
$$

Assim, utilizando as técnicas desenvolvidas anteriormente, fortalecemos o resultado de Culik demonstrando que:

$$
\begin{aligned}
& \text { "Para cada linguagem recursivamente enume- } \\
& \text { rävel } L \text {, existem efetivamente um morfismo } \\
& h_{0} e \text { códigos } h_{1} e h_{2} \text { som } h_{1} \text { de atraso um } \\
& \text { da esquerda para a direita e } h_{2} \text { da direi- } \\
& \text { ta para a esquerda tais que } L=\left(e q\left(h_{1}, h_{2}\right)\right) h_{0} \text {. }
\end{aligned}
$$


Desse modo, este trabalho é composto de cinco capitu los que descrevemos resumidamente a seguir.

No Capitulo 1 apresentamos os conceitos básicos que serão utijizados no decorrer de todo o texto.

o Capituzo 2 é dedicado inteiramente ao estudo das mäquinas de Turing e chegamos a construir para cada máquina de Turing determinística uma outra mäquina de Turing reversỉvele normalizada que è equivalente.

No Capituzo 3 demonstramos a indecidibilidade do Pro blema de Correspondência de Post quando restrito a códigos de atraso um. Exibimos tambëm alguns casos em que o Problema de Correspondência de Post foi provado ser indecidivel, outros em que è decidível e alguns que ainda permanecem em aberto.

o objetivo do Capituzo 4 é apresentar a representação de linguagens recursivamente enumeräveis como imagens homomór ficas de conjuntos-igualdade minimais de cödigos, sendo que um deles é de atraso um da esquerda para a direita e o outro de atraso um da direita para a esquerda. Além disso, exibimos algumas outras representações de linguagens recursivamente enu meráveis obtidas por A.Salomaa (1979), K.Culik e H.A.Maurer (1979) e J.Engelfriet e G.Rozenberg (1980).

Finalmente, no Capitulo 5 apontamos algumas consequên cias dos resultados obtidos para conjuntos-igualdade de códigos, especialmente para aqueles que são regulares.

E, por ültimo, apresentamos as Referências Bibliogrä ficas, o Indice de Simbolos e o Indice de Definifões. 



\section{CAPITULO 1}

\section{MORFISMOS E CONJUNTOS-IGUALDADE DE MORFISMOS}

\section{DE MONOTIDES LIVRES}

Neste trabalho, vamos supor que o leitor seja familiarizado com os conceitos de relacões, funcões (parciais), mo nöides (livres), morfismos de monöides livres e, em geral,com a Teoria de Linguagens Formais, principalmente no que se refe re a linguagens recursivamente enumeráveis e problemas de decidibilidade, como exposto, por exemp1o, por Imre Simon (1981), Salomaa (1973) e por Hopcroft e U11man (1969).

Recordamos aqui a notação relativa a funções e composição de funções que utilizaremos neste texto.

Seja $f: A \longrightarrow B$ uma funcão. Então, para cada a em A, a imagem de a por $f \vec{e}$ denotada por af e se af $\overrightarrow{\mathrm{e}}\{\mathrm{b}\}$, para $\underline{1}$ 
gum b em B, escrevemos apenas af=b. Ou seja, no decorrer des te texto, não faremos distinção para denotar um conjunto unitảrio e seu único elemento.

Se $g: B \longrightarrow C$ é uma outra função, então a composicão. de $f$ com $g$, denotada por $\mathrm{fg}, \mathrm{fg}: \mathrm{A} \longrightarrow \mathrm{C}$, é definida, para cada a em A, por

$$
a(f g)=(a f) g \text {. }
$$

Assim, denotamos a imagem de a por fg simplesmente por afg.

O nosso objetivo neste capitulo ë apresentar as defi nições e as propriedades de algumas restrições importantes de morfismos e introduzix os conjuntos-igualdade (minimais) de morfismos de monóides livres.

Em seguida, verificamos que a classe dos conjuntos-igualdade minimais de morfismos injetores está propriamente contida na classe dos conjuntos-igualdade minimais de morfismos quaisquer.

0 estudo dos conjuntos-igualdade de morfismos de monóides livres que são regulares serä feito no Capítulo 5.

1. MORFISMOS DE MONÓIDES LIVRES

Inicialmente, vamos enunciar a propriedade fundamental dos monöides livres: 
"Seja M um monóide e seja $f: \Sigma \longrightarrow$ uma função.

Existe uma única extensão de $f$ a um morfismo $f^{*}: \Sigma^{*} \longrightarrow M^{\prime \prime}$.

A demonstração pode ser encontrada em Lucchesi, Simon, Simon, Simon e Kowaltowski (1979 - capítulo D.I) . Logo, devido a esta propriedade, sempre que for necessảrio construir um morfismo $f: \Sigma^{*} \longrightarrow M$, vamos somente especificar os va lores de of, para cada o em $\Sigma$.

A seguir apresentamos algumas definições e notaçōes de natureza mais especifica.

Seja $h: \Gamma^{*} \longrightarrow \Delta^{*}$ um morfismo injetor. Nesse caso, chamamos h de cödigo.

Dizemos então que o código h è de atraso $k$ da esquer̂ da para a direita (da direita para a esquerá) se existir um natural $k$ tal que

$$
\begin{aligned}
& \text { para cada } u \text { em } \Gamma h, v \text { em }(\Gamma h)^{k} \text { e w em } \Delta^{*}, \\
& \text { se uvw } \in(\Gamma h)^{*} \quad\left(w v u \in(\Gamma h)^{*}\right) \\
& \text { então vw } \in(\Gamma h)^{*}\left(\text { wv } \in(\Gamma h)^{*}\right) .
\end{aligned}
$$

$\underline{\text { EXEMPLO } 1}-$ Seja o morfismo $h:\left\{\sigma_{1}, \sigma_{2}, \sigma_{3}\right\} * \rightarrow\{a, b\} *$, definido por:

$$
\sigma_{1} h=a \quad, \sigma_{2} h=b a \quad \text { e } \quad \sigma_{3} h=b b .
$$

Então, h è de atraso zero da esquerda para a direita, mas não 
$\ddot{e}$ de atraso $k$ da direita para a esquerda, para nenhum $k \geq 0$.

Se um cödigo h è de atraso $k$ em ambos os sentidos, dizemos simplesmente que h ê de atraso $k$.

EXEMPLO 2 - Seja o morfismo $h:\left\{\sigma_{1}, \sigma_{2}, \sigma_{3}\right\} * \rightarrow\{a, b\} *$, definido por:

$$
\sigma_{1} h=a b \quad, \quad \sigma_{2} h=a b b \quad \text { e } \quad \sigma_{3} h=b a a b .
$$

Nesse caso, h é de atraso um em ambos os sentidos.

Dizemos que um código hè de atraso limitado (da direita para a esquerda ou da esquerda para a direita) se ele for de atraso $k$ (da direita para a esquerda ou vice-versa, res pectivamente), para algum $k \geq 0$.

Um morfismo $h: \Gamma^{*} \longrightarrow \Delta^{*}$ é dito prefixo (sufixo) se para cada $x$ e $y$ em $r$,

$$
\begin{aligned}
& \text { se } x h=(y h) z \quad(x h=z(y h)) \text {, para algum } z \text { em } \Delta^{*}, \\
& \text { então } x=y \quad \text { e } z=1 .
\end{aligned}
$$

EXEMPLO 3 - 0 morfismo h do exemplo 1 è um prefixo.

$$
\text { Seja } f:\left\{\sigma_{1}, \sigma_{2}, \sigma_{3}, \sigma_{4}, \sigma_{5}\right\}^{*} \longrightarrow\{a, b\} * \text { um morfismo dado }
$$

por:

$$
\sigma_{1} f=a a, \sigma_{2} f=a b, \sigma_{3} f=b a a, \sigma_{4} f=b a b \text { e } \sigma_{5} f=b b .
$$

Então, f è um morfismo prefixo. 
EXEMPLO $4-0$ morfismo $h:\left\{\sigma_{1}, \sigma_{2}, \sigma_{3}, \sigma_{4}\right\}^{*} \rightarrow\{a, b, c\} *$ definido por:

$$
\sigma_{1} h=b, \sigma_{2} h=b a, \sigma_{3} h=c a \text { e } \sigma_{4} h=c
$$

é um sufixo.

Se um morfismo h è simultaneamente prefixo e sufixo, dizemos que h $\ddot{\mathrm{e}}$ um biprefixo.

EXEMPLO 5 - Os morfismos $f:\left\{\sigma_{1}, \sigma_{2}\right\} * \rightarrow\{a, b\} * e$ $g:\left\{\sigma_{1}, \sigma_{2}, \sigma_{3}\right\} * \longrightarrow\{a, b\} *$ definidos, respectivamente, por:

$$
\begin{aligned}
& \sigma_{1} f=a \quad \text { e } \sigma_{2} f=b, \\
& \sigma_{1} g=a^{2}, \sigma_{2} g=a b a \text { e } \sigma_{3} g=b
\end{aligned}
$$

são morfismos biprefixos.

Podemos observar que:

- todo morfismo prefixo, sufixo ou biprefixo é injetor;

- todo morfismo prefixo (sufixo) ê de atraso zero da esquerda para a direita (da direita para a esquerda);

- todo morfismo biprefixo è de atraso zero (em ambos os senti dos )

Vamos denotar por $P(S)$ a classe dos morfismos prefixos (sufixos). Então, PS(SP) designa a classe dos morfismos que são composigões de um prefixo (sufixo) com um sufixo (pre 
fixo). Assim, $P \cap S$ representa a classe dos morfismos bipre ficos e PS $\cap$ SP a classe dos morfismos que são tanto composicões de um prefixo com um sufixo como de um sufixo com um pre fixo.

Com relação aos morfismos prefixo, sufixo, biprefixo e de atraso limitado, uma referência importante é Perrin(1980).

Se h é um morfismo de $\Gamma^{*}$ em $\Delta^{*}$ e $\Sigma$ é um subconjunto de $\Gamma$, então dizemos que $h$ preserva $\Sigma$ sse

$$
\begin{aligned}
& \text { para cada o em } \Sigma, \quad \sigma h=\sigma \\
& \text { e para cada } \sigma \text { em } \Gamma \backslash \Sigma, \sigma h=1 \text {. }
\end{aligned}
$$

Vamos denotâ-10 por $\mathrm{h}_{\Sigma}$.

0 morfismo identidade sobre $r *$ será denotado por $1_{\Gamma}$ * Chamamos um morfismo $\mathrm{h}: \mathrm{r}^{*} \longrightarrow \Delta^{*}$ de elementar se não existem um alfabeto $\Sigma$, com $|\Sigma|<|\Gamma|$ e dois morfismos $f: I^{*} \longrightarrow \Sigma^{*}$ e $g: \Sigma^{*} \longrightarrow \Delta^{*}$ tais que $h=f g$.

EXEMPLO $6-0$ morfismo $h:\{a, b, c, \alpha, \beta, \gamma\}^{*} \longrightarrow\{a, b, c, d, e, f\}^{*}$, definido por:

$$
a h=d e, b h=d f e, c h=d f f e, \alpha h=a, \beta h=b c \text { e } \gamma h=b a
$$

è elementar.

Observemos que todo morfismo elementar é injetor.

Um resultado importante que relaciona morfismos elementares com códigos de atraso limitado é o seguinte. 
PROPOSICATO 1 - Todo morfismo elementar ë um cỏdigo de atraso limitado.

A demonstração pode ser encontrada em Courcelle(1978) ou em Rozenberg e Salomaa (1980).

2. O CONJUNTO-IGUALDADE (MINIMAL) DE MORFISMOS DE MONOIDES LIVRES

Sejam os monóides livres $r *$ e $\Delta^{*}$ gerados, respectiva mente, pelos alfabetos finitos $r$ e $\Delta$. Sejam os morfismos $f, g: \Gamma^{*} \longrightarrow \Delta^{*}$.

0 conjunto-igualdade de $f e g$ consiste de todas as palavras $w^{\text {em }} \Gamma^{*}$ que têm a mesma imagem por $f$ e por $g$, ou seja,

$$
\mathrm{Eq}(f, g)=\left\{w \in \Gamma^{*} / w f=w g\right\} .
$$

(A inclusão de $\mathrm{Eq}(f, g)$ em $\Gamma^{*}$ constitui o que se deno mina equalizador em Teoria das Categorias. Mas, preferimos adotar o termo conjunto-igualdade jä que este tem sido o pa-drão em todos os textos referentes a esse assunto.)

EXEMPLO 7 - Sejam os morfismos $f, g:\{a, b, c\} * \longrightarrow\{a, b, c, d\}^{*}$, definidos pela tabela abaixo: 


\begin{tabular}{|c|ccc|}
\hline$\gamma$ & $a$ & $b$ & $c$ \\
\hline$\gamma f$ & $a$ & $b c$ & $b d$ \\
\hline$\gamma g$ & $a b$ & $c b$ & $d$ \\
\hline
\end{tabular}

Então, Eq $(f, g)=\left(a b^{*} c\right) *$.

EXEMPLO 8 - Sejam os morfismos $f_{1}, g_{1}, f_{2}$ e $g_{2}:\{a, b, c\}^{*} \rightarrow\{a\} *$, dados pelas tabelas abaixo:

\begin{tabular}{|l|ll|}
\hline$r$ & $a$ & $b$ \\
\hline$r f_{1}$ & a & aa \\
\hline$r g_{1}$ & aa & a \\
\hline
\end{tabular}

\begin{tabular}{|l|ll|}
\hline$\gamma$ & $a$ & $b$ \\
\hline$\gamma f_{2}$ & $a$ & 1 \\
\hline$\gamma g_{2}$ & 1 & $a$ \\
\hline
\end{tabular}

Então, ë fäcil ver que

$E_{q}\left(f_{1}, g_{1}\right)=\left\{x \in\{a, b\} * /|x|_{a}=|x|_{b}\right\}=E q\left(f_{2}, g_{2}\right)$.

EXEMPLO 9 - Sejam os morfismos $f, g:\{a, b, c\}^{*} \longrightarrow\{a, c\} *$ definidos pela babela abaixo:

\begin{tabular}{|c|ccc|}
\hline$\gamma$ & $a$ & $b$ & $c$ \\
\hline$\gamma f$ & $a$ & $a$ & $c^{2}$ \\
\hline$\gamma g$ & $a^{2}$ & $c$ & $c$ \\
\hline
\end{tabular}

Logo, pode-se verificar que

Eq $(f, g)=\left(\left\{a^{n} b^{n} c^{n} / n \geq 0\right\} \quad U\left\{c^{n} b^{n} a^{n} / n \geq 0\right\}\right) *$ 
Os conjuntos-igualdade de morfismos têm sido de gran de importância na solução de alguns problemas da Teoria de Lin guagens Formais, Por exemplo, a decidibilidade do Problema da Equivalência da Sequência para os sistemas DOL foi demonstrada originariamente por Culik e Fris (1977) e então, utilizando os conjuntos-igualdade, Ehrenfeucht e Rozenberg (1978) apresentaram uma demonstração mais simples desse resultado.

Mais recentemente, algumas representações de lingua gens recursivamente enumeräveis, utilizando conjuntos-igualda de de morfismos de monóides livres, têm sido apresentadas.

Para maiores considerações sobre a classe dos conjun tos-igualdade, denotada por Eq(HOM), onde HOM é a classe dos morfismos, o leitor poderá consultar Salomaa (1978), Enge1friet e Rozenberg (1979 e 1980) e Rozenberg e Salomaa (1980, capítulo III).

$\mathrm{Na}$ sequência, vamos definir o conjunto-igualdade minimal de dois morfismos de monóides livres.

Sejam os morfismos $f, g: \Gamma^{*} \longrightarrow \Delta^{*}$.

0 conjunto-igualdade minimal de $f e g$ consiste de to das as palavras $w$ em $\Gamma^{*}$ que têm a mesma imagem por $f$ e por $g$, mas que não têm nerhum segmento inicial pröprio com imagens $\underline{i}$ guais por $f$ e por $g$, ou seja,

$$
\begin{gathered}
\text { eq }(f, g)=\left\{w \in \Gamma^{+} / \text {wf }=w g \text { e se } w=u v \text {, para } u \text { e } v\right. \\
\text { em } \left.\Gamma^{+} \text {, então uf } \neq u g\right\} .
\end{gathered}
$$


EXEMPLO 10 - Considerando-se os morfismos $f$ e g do exemplo 7 , temos que eq $(f, g)=a b^{*} c$.

Se $f$ e g são os morfismos do exemplo 9 , então

$$
\text { eq }(f, g)=\left\{a^{n_{b}}{ }^{n} c^{\grave{n}} / n>0\right\} U\left\{c^{n_{b}} b_{a}^{n} / n>0\right\} .
$$

Denotando-se por IHOM a classe dos morfismos injetores, seja Eq(IHOM) a classe dos conjuntos-igualdade de morfis mos injetores. Engelfriet e Rozenberg (1979) mostraram o seguinte resultado.

TEOREMA $1-\mathrm{Eq}$ (IHOM) $\underset{\neq}{\subsetneq} \mathrm{Eq}(\mathrm{HOM})$.

A fim de demonsträ-1o, necessitamos de outros resultados conhecidos que apresentamos a seguir através de dois le mas.

LEMA 1 - Sejam as palavras $x$ e $y$ tais que $x y=y x$. Então, existe uma palavra $z$ tal que $x$ e $y$ pertencem a $z^{*}$.

DEMONSTRAÇÃO - Por indução sobre $|x|+|y|$.

Se $|x|+|y|=0$ então $x=y=1$ e o resultado é trivial.

Suponhamos que o resultado seja válido para palavras $\mathrm{x}$ e $y, \operatorname{com}|x|+|y| \leq k$, para algum $k \geq 1$.

Sejam as palavras $x$ e $y$ tais que $|x|+|y|=k+1$ e $x y=y x$. Então, ou $x$ é um segmento inicial de $y$ ou $y$ é um seg mento inicial de $x$. Sem perda de generalidades, vamos supor 
que $x \vec{e}$ um segmento inicial de $y$.

Se $x=1$, o resultado $\overrightarrow{\mathrm{e}}$ trivial.

Suponhamos então que $x$ è uma palavra não vazia. Logo, existe uma palavra u tal que $x u=y$ e $|u|<|y|$. Mas, de $x y=y x$ segue que $x x u=x u x e$, consequentemente, $x u=u x$. Como $|x|+|u|<|x|+|y|$, segue pela hipótese da indução que existe uma palavra não vazia $z$ tal que $x$ e $u$ pertencem a $z^{*}$. $P_{0}$ rêm, $y=x u$. Portanto, $y$ tambëm pertence a $z^{*}$.

LEMA 2 - Sejam as palavras $x, y$ e $v$.

(i) Se $x \neq 1$ e existem infinitos inteiros não-negativos n tais que $x^{n} \vec{e}$ um segmento inicial ảe $y^{n}$ então $x^{\mathbb{N}}=y^{\mathbb{N}}$.

(ii) $\quad$ Se $x^{\mathbb{N}}=y^{\mathbb{N}}$ então existe uma palavra $z$ tal que $x$ e $y$ pertencem a $z^{*}$.

(iii) Se $x$ pertence a $y^{+} \cap v^{+}$então existe uma palavra $z$ tal que $x, y \in v$ pertencem a $z$.

\section{DEMONSTRAÇÃO -}

(i) E óbvia.

(ii) $\quad$ Se $x^{\mathbb{N}}=y^{\mathbb{N}}$ então ou $x$ é um segmento inicial de $y$ ou vice-versa. Sem perder a generalidade, podemos supor que $x$ è um segmento inicial de $y$. Logo, existe uma pa lavra $v$ tal que $x v=y$. Então, $x^{\mathbb{N}}=y^{\mathbb{N}}=(x v)^{\mathbb{N}}$. Logo, $x^{\mathbb{N}}=(v x)^{\mathbb{N}}$. Portanto, $(x v)^{\mathbb{N}}=(v x)^{\mathbb{N}}$ e assim, $\quad x v=v x$. Pelo Lema 1 segue que existe uma palavra $z$ tal que $x$ 
e $v$ pertencem a $z^{*}$. Consequentemente, $x$ e y pertencem a $z^{*}$.

(iii) Se $x$ pertence a $y^{+} n v^{+}$então $x$ pertence $a y^{+}$e a $v^{+}$. Logo, $y^{\mathbb{N}}=v^{\mathbb{N}}$. Então, resulta de (ii) que existe uma palavra $z$ tal que $y$ e $v$ pertencem a $z^{*}$. Assim, $x, y e$ $v$ pertencem a $z^{*}$.

啳

DEMONSTRACAO DO TEOREMA 1 -

Queremos mostrar que $\mathrm{Eq}(\mathrm{IHOM}) \underset{\neq}{\subset} \mathrm{Eq}(\mathrm{HOM})$.

Sejam $\alpha$ e $B:\{a, b, c, d\} * \rightarrow\{a, c\}^{*}$ os morfismos definidos pela tabela abaixo:

\begin{tabular}{|c|cccc|}
\hline$\sigma$ & $a$ & $b$ & $c$ & $d$ \\
\hline$\sigma \alpha$ & $a$ & $a$ & $c^{2}$ & 1 \\
\hline$\sigma \beta$ & $a^{2}$ & $c$ & $c$ & 1 \\
\hline
\end{tabular}

Entäo, $\operatorname{Eq}(\alpha, \beta)=\left(\left\{\left(d^{*} a d^{*}\right)^{n}\left(d^{*} b d^{*}\right)^{n}\left(d^{*} c d^{*}\right)^{n} / n>0\right\} \quad U\right.$

$$
\left.\left\{\left(d^{*} c d^{*}\right)^{n}\left(d^{*} b d^{*}\right)^{n}\left(d^{*} a d^{*}\right)^{n} / n>0\right\}\right) * U d^{*} .
$$

Mas, a e $\beta$ não são morfismos injetores.

Suponhamos, por absurdo, que existam morfismos $f$ e $g$ injetores tais que $\mathrm{Eq}(f, g)=\mathrm{Eq}_{\mathrm{q}}(\alpha, \beta)$.

Como $d \in E q(\alpha, \beta)$, segue que

$$
\mathrm{df}=\mathrm{dg} \text {. }
$$

Podemos observar que $d f \neq 1$, pois, caso conträrio, $f$ e 
g não seriam injetores.

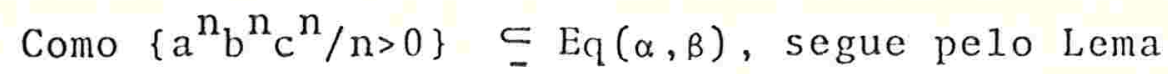
que $(a f)^{\mathbb{N}}=(a g)^{\mathbb{N}}$. Então, pe1o Lema $2(i i)$ existe uma palavra não vazia $z_{1}$ tal que

$$
\text { af e ag } \in z_{1}^{+} \text {. }
$$

Analogamente, porque $\left\{c^{n} b^{n} a^{n} / n>0\right\} \subseteq E q(\alpha, \beta)$, existe uma palavra não vazia $z_{2}$ tal que

$$
\text { cf e } \operatorname{cg} \in \mathrm{z}_{2}^{+} \text {. }
$$

Como af $\neq \mathrm{ag}$, pois a $\notin \mathrm{Eq}(\mathrm{f}, \mathrm{g})$, podemos supor, sem perder a generalidade, que

af $\hat{e}$ um segmento inicial de ag.

Como $\left\{a^{n} d^{m} b^{n} c^{n} / n, m>0\right\} \subseteq E q(\alpha, \beta)$, resulta de (2) e Lema $2(i)$ que $(d f)^{\mathbb{N}}=z_{1}^{\mathbb{N}}$. Então, pelo Lema $2(i i)$ existe uma palavra não vazia $z_{3}$ tal que df e $z_{1} \in z_{3}^{+}$. Logo, de (1) e (2) concluimos que

$$
\text { af, ag, df e dg } \in z_{3}^{+} \text {. }
$$

Analogamente, porque $\left\{c^{n} d^{m} b^{n} a^{n} / n, m>0\right\} \subseteq E q(\alpha, \beta)$, segue que $(\mathrm{d} f)^{\mathbb{N}}=\mathrm{z}_{2}^{\mathbb{N}}$ e pelo Lema $2(i i)$ existe uma palavra não vazia $z_{4}$ tal que $\mathrm{df}$ e $z_{2} \in \mathrm{z}_{4}^{+}$. Logo, de (1) e (3) concluímos que

$$
c f, c g, \text { df } e \text { dg } \in z_{4}^{+} \text {. }
$$


Então, de (5) e (6) e Lema 2 (iii) resulta que existe uma palavra não vazia $z_{5}$ tal que

$\mathrm{af}, \mathrm{ag}, \mathrm{cf}, \mathrm{cg}, \mathrm{df}$ e dg $\in \mathrm{z}_{5}^{+}$.

Temos dois casos a considerax:

19) $|c f|>|c g|$.

De (4) e (7) temos que

$$
\begin{aligned}
\text { ag } & =(a f) z_{5}^{k}, \text { para algum } k \geq 1 \\
\text { e } \quad c f & =z_{5}^{\ell}(c g), \text { para algum } \ell \geq 1 .
\end{aligned}
$$

Sejam af $=z_{5}^{r}$ e $c g=z_{5}^{s}$, para $r$ e $s \geq 1$. Então, $\left(a^{l} c^{k}\right) g=z_{5}^{(r+k) l+k s}=z_{5}^{r l+(\ell+s) k}=\left(a^{\ell} c^{k}\right) f$.

o que implica que $a^{l} c^{k} \in E q(f, g)=E q(\alpha, \beta)$; um absurdo.

2) $|c f|<|c g|$.

Como $\left\{a^{\left.n_{b}{ }^{n} c^{n} / n>0\right\}} \subseteq E q(\alpha, \beta)\right.$, de (4) e Lema 2(i) obtemos que $(b f)^{\mathbb{N}}=(a g)^{\mathbb{N}}$.

Então, pelo Lema $2(i i)$ existe uma palavra não vazia $z_{6}$ tal que bf e ag $\in z_{6}^{+}$e, utilizando (7) $\odot$ o Lema 2(iii) se gue que existe uma palavra não vazia $z_{7}$ tal que ag, $z_{5}$ e $z_{6} \in$ $z_{7}^{\dot{r}}$.

Logo, resulta de (7) que 
af, ag, cf, cg, df, dg e bf $\in z_{7}^{+}$.

Assim, para cada $n>0$,

$$
\left(a^{n} b^{n} c^{n}\right) f, a^{n} g \quad \text { e } \quad c^{n} g \quad \in \quad z_{7}^{+} .
$$

Como $\left(a^{n} b^{n} c^{n}\right) f=\left(a^{n} b^{n} c^{n}\right) g$, para cada $n>0$, segue que $b^{n} g \in z_{7}^{+}$.

Consequentemente, $(\mathrm{bg})^{\mathbb{N}}=z_{7}^{\mathbb{N}}$ e pelo Lema $2($ ii) existe uma palavra não vazia $z$ tal que bg e $z_{7} \in z^{+}$.

Portanto, de (8) resulta que

$$
\text { af, bf, cf, df, ag, bg, cg e dg } \in z^{+} \text {. }
$$

Podemos notar ainda que $|b f|>|b g|$, jả que $|a f|<$ $|a g|,|c f|<|c g|$ e $(a b c) f=(a b c) g$.

Então, de (4) e (9) temos que

$$
\begin{aligned}
\text { ag } & =(\mathrm{af}) \mathrm{z}^{\mathrm{k},}, \operatorname{para} \text { algum } k \geq 1 \\
\mathrm{e} \quad \mathrm{bf} & =\mathrm{z}^{\ell}(\mathrm{bg}), \text { para algum } \ell \geq 1 .
\end{aligned}
$$

Sejam af $=z^{r}$ e $b g=z^{s}$, para $r$ e $s \geq 1$. Logo, $\left(a^{\ell} b^{k}\right) g=z^{(x+k) l+s k}=z^{r \ell+(l+s) k}=\left(a^{\ell} b^{k}\right) f$

0 que implica que $a^{2} b^{k} \in E q(f, g)=E q(\alpha, \beta) ;$ um absurdo.

Desse modo, temos provado que $\mathrm{Eq}_{\mathrm{q}}(\mathrm{IHOM}) \underset{\neq}{\subset} \mathrm{Eq}_{\mathrm{q}}(\mathrm{HOM})$. 
Esse resultado continua välido para a classe dos con juntos-igualdade minimais, is to é,

COROLARIO $1-$ eq (IHOM) $\underset{\neq}{\subsetneq}$ eq (HOM).

DEMONSTRACÃO - Segue imediatamente do Teorema 1 e do fato que para cada par de morfismos $f$ e $g$, eq(f,g) é o ünico gerador minimal de $E_{q}(f, g)$, ou seja,

$$
\begin{aligned}
& (\mathrm{eq}(f, g)) *=E_{q}(f, g) \\
\text { e } \quad & \text { eq }(f, g)=\left(E_{q}(f, g) \backslash\{I\}\right) \backslash\left(E_{q}(f, g) \backslash\{1\}\right)^{2} .
\end{aligned}
$$




\section{CAPITULO 2}

\section{MARQUINAS DE TURING}

Neste capítulo, apresentamos, inicialmente, uma definição de máquina de Turing e descrevemos o seu funcionamento, utilizando um formalismo inspirado no livro de Eilenberg (1974). O formalismo que adotamos foi fundamental para a demonstração dos resultados principais deste trabalho.

Em seguida, introduzimos máquinas de Turing (semi) normalizadas e reversíveis. E, então, para cada máquina de Turing deterministica associamos uma mäquina de Turing reversível e normalizada equivalente.

Finalmente, construímos o acoplamento de uma mäquina de Turing reversível e normalizada com a sua reversa. 
1. DEFINIC AO

Uma máquina de Turing A consiste de:

- duas fitas que serão referidas como fita à esquerda e fita à direita;

- um conjunto finito $Q_{A}$, cujos elementos são chamados de esta dos;

- um alfabeto finito $\mathrm{T}_{A}$, chamado de alfabeto de trabalho;

- um subconjunto $\Sigma_{A}$ de $T_{A}$, chamado de arfabeto de entrada;

- subconjuntos $I_{A}$ e $F_{A}$ de $Q_{A}$, cujos elementos são denominados, respectivamente, de estados iniciais e finais;

- um subconjunto $A_{A}$ de $Q_{A} \times E_{A} \times D_{A} \times Q_{A}$, onde $E_{A}\left(D_{A}\right)$ e oconjunto de instruçoes que agem sobre o conteúdo da fita à esquerda (direita) de A.

$$
E_{A}=\left\{E_{\sigma} / \sigma \in T_{A}\right\} \cup\left\{E_{\sigma}^{-1} / \sigma \in T_{A}\right\} \cup\{V, N\}
$$

e

$$
D_{A}=\left\{D_{\sigma} / \sigma \in T_{A}\right\} \cup\left\{D_{\sigma}^{-1} / \sigma \in T_{A}\right\} U\{V, N\} .
$$

Os elementos de $A_{A}$ são chamados de arestas Se $\alpha=\left(q, i_{1}, i_{2}, p\right)$ è una aresta, então $p$ e q são ditos a origem e o término de $\alpha$, respectivamente.

Para cada $q$ em $Q_{A}$, denotamos por $A_{A}(q)$ o subconjunto de $A_{A}$ contendo todas as arestas com origem em $q$, ou seja,

$$
A_{A}(q)=\left\{\alpha \in A_{A} / \alpha \text { tem origem em } q\right\} .
$$


As máquinas de Turing tambēm podem ser representadas graficamente, como segue.

\section{EXEMPLO 1}

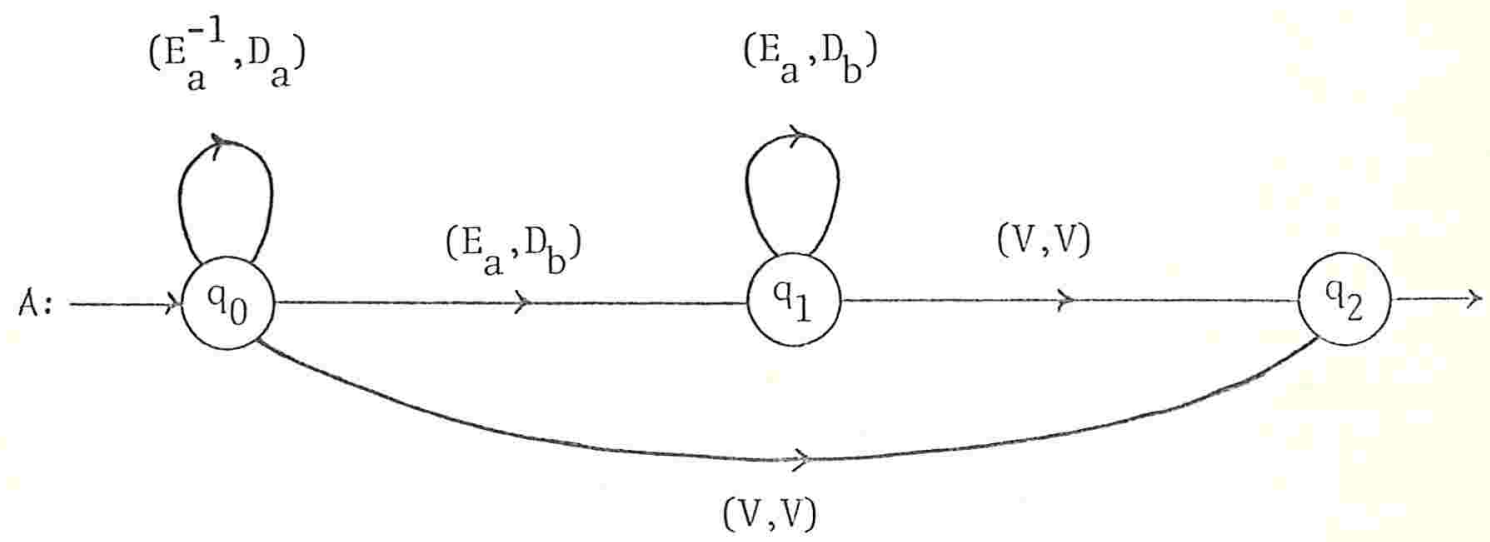

onde $Q_{A}=\left\{q_{0}, q_{1}, q_{2}\right\}$,

$$
\begin{aligned}
& \mathrm{T}_{A}=\Sigma_{A}=\{\mathrm{a}, \mathrm{b}\}, \\
& \mathrm{I}_{A}=\left\{\mathrm{q}_{0}\right\}, \\
& \mathrm{F}_{A}=\left\{\mathrm{q}_{2}\right\} \\
& \mathrm{A}_{A}=\left\{\left(\mathrm{q}_{0}, \mathrm{E}_{\mathrm{a}}^{-1}, \mathrm{D}_{\mathrm{a}}, \mathrm{q}_{0}\right),\left(\mathrm{q}_{0}, \mathrm{E}_{\mathrm{a}}, \mathrm{D}_{\mathrm{b}}, \mathrm{q}_{1}\right),\left(\mathrm{q}_{0}, \mathrm{v}, \mathrm{v}, \mathrm{q}_{2}\right),\right. \\
&\left.\left(\mathrm{q}_{1}, \mathrm{E}_{\mathrm{a}}, \mathrm{D}_{\mathrm{b}}, \mathrm{q}_{1}\right),\left(\mathrm{q}_{1}, \mathrm{v}, \mathrm{v}, \mathrm{q}_{2}\right)\right\} .
\end{aligned}
$$

2. DESCRIÇÃO DO FUNCIONAMENTO

A seguir, descrevemos informalmente o funcionamento de uma mảquina de Turing (em termos de suas computações ) 
e, ao mesmo tempo, introduzimos formalmente alguns conceitos.

Uma máquina de Turing A possui um controle finito que se encontra, a cada instante, num dos estados q em $Q_{A}$ e cada uma de suas fitas contēm uma palavra $s_{j} e m T_{A}^{*}, j=1$ e 2 . Assim, denotamos a configuração das fitas de $A$ pelo par $\left(\mathrm{s}_{1}, \mathrm{~s}_{2}\right)$, onde $\mathrm{s}_{1}\left(\mathrm{~s}_{2}\right)$ è o conteúdo da fita à esquerda (direita) e descrevemos a configuração instantânea de $A$ por $\varepsilon s_{1} \mathrm{qs}_{2} \varepsilon$, onde $\varepsilon$ é um símbolo não pertencente a $\mathrm{T}_{A}$.

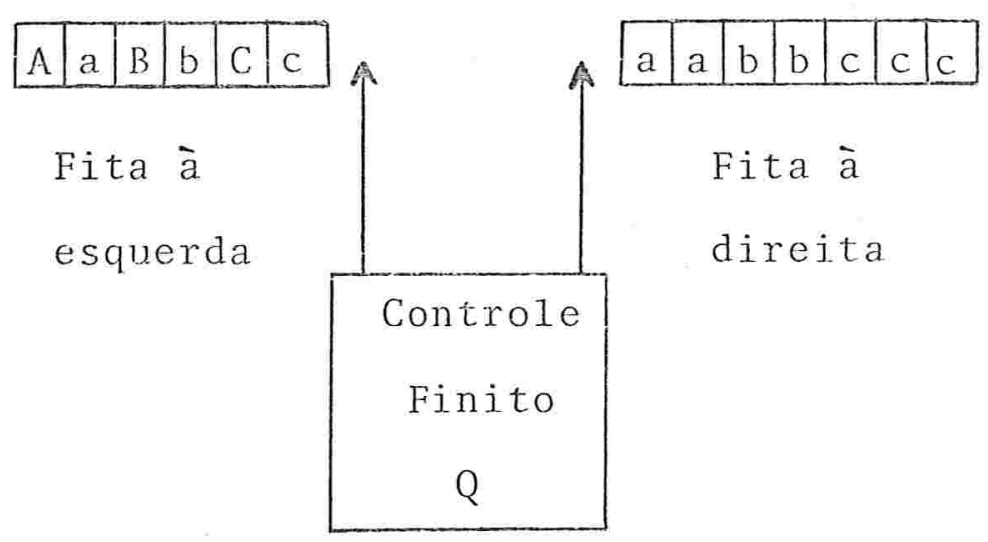

A execução de uma instrução em $\mathrm{D}_{A}\left(\mathrm{E}_{A}\right)$ pode retirar ou acrescentar uma letra na extremidade esquerda (direita) da fita à direita (esquerda) ou não alterar o seu conteúdo.

Cada instrução em $E_{A} U D_{A}$ define uma função parcial de $\mathrm{T}_{A}^{*}$ em $\mathrm{T}_{A}^{*}$ como segue: para cada $\mathrm{s}$ em $\mathrm{T}_{A}^{*}$ e $\sigma$ em $\mathrm{T}_{A}$, $-\mathrm{sN}=\mathrm{s}$

isto è, a instrução $\mathrm{N}$ deixa o conteüdo da fita (à esquerda ou à direita) inalterado; $N$ é a função identidade ${ }^{1} \mathrm{~T}_{A}^{*}$ sobre $\mathrm{T}_{A}^{*}$; 
$-s V= \begin{cases}1, & \text { se } \quad s=1 \\ \emptyset & \text { caso contrário }\end{cases}$

isto é, a instrução. V também não altera o conteúdo da fita (à esquerda ou à direita), mas só pode ser executada se a fita estiver vazia;

$$
\begin{aligned}
& -\mathrm{sD}_{\sigma}= \begin{cases}t, & \text { se } s=\sigma t, \text { para algum } t \text { em } \mathrm{T}^{*} \\
\emptyset, & \text { caso conträrio }\end{cases} \\
& \text { - } \mathrm{SE}_{\sigma}= \begin{cases}t, & \text { se } s=t \sigma, \text { para algum } t \text { em } \mathrm{T}_{A}^{*} \\
\emptyset, & \text { caso conträrio }\end{cases}
\end{aligned}
$$

isto $\vec{e}$, a instrução $D_{\sigma}\left(E_{\sigma}\right) l \hat{e}$ o simbolo o se este for a primeira letra da extremidade esquerda (direita) do conteúdo da fita à direita (esquerda); caso contrário, ela não pode ser executada;

$$
\begin{aligned}
& -\mathrm{sD}_{\sigma}^{-1}=\sigma s \\
& -\mathrm{sE}_{\sigma}^{-1}=s \sigma
\end{aligned}
$$

ou seja, a instrução $\mathrm{D}_{\sigma}^{-1}\left(\mathrm{E}_{\sigma}^{-1}\right)$ grava o símbolo o na extremidade esquerda (direita) da fita à direita (esquerda).

Os domínios e as imagens das funções parciais descri tas acima são dados por: 


$$
\begin{array}{ll}
\operatorname{dom} N=\mathrm{T}_{A}^{*} & \text { im } N=\mathrm{T}_{A}^{*} \\
\text { dom } \mathrm{V}=\{1\} & \text { im } \mathrm{V}=\{1\}
\end{array}
$$

para cada $\sigma$ em $\mathrm{T}_{A}$, dom $\mathrm{D}_{\sigma}=\sigma \mathrm{T}_{A}^{*} \quad$ im $\mathrm{D}_{\sigma}=\mathrm{T}_{A}^{*}$

$$
\begin{array}{ll}
\operatorname{dom} \mathrm{E}_{\sigma}=\mathrm{T}_{A}^{*} \sigma & \text { im } \mathrm{E}_{\sigma}=\mathrm{T}_{A}^{*} \\
\operatorname{dom} \mathrm{D}_{\sigma}^{-1}=\mathrm{T}_{A}^{*} & \text { im } \mathrm{D}_{\sigma}^{-1}=\sigma \mathrm{T}_{A}^{*} \\
\operatorname{dom} \mathrm{E}_{\sigma}^{-1}=\mathrm{T}_{A}^{*} & \text { im } \mathrm{E}_{\sigma}^{-1}=\mathrm{T}_{A}^{*} \sigma .
\end{array}
$$

Observemos que $\mathrm{E}_{\sigma}$ e $\mathrm{E}_{\sigma}^{-1}$ ( $\mathrm{D}_{\sigma}$ e $\mathrm{D}_{\sigma}^{-1}$ ) são relações inversas.

Tambèm são vâlidas as seguintes identidades:

$$
\begin{aligned}
& \mathrm{VV}^{-1}=\mathrm{V}^{-1} \mathrm{~V}=\mathrm{V}=\mathrm{V}^{-1} \text { e } \mathrm{NN}^{-1}=\mathrm{N}^{-1} \mathrm{~N}=\mathrm{N}=\mathrm{N}^{-1} . \\
& \mathrm{E}, \text { para cada } \sigma \text { em }^{\mathrm{T}} \mathrm{A}, \\
& \mathrm{D}_{\sigma}^{-1} \mathrm{D}_{\sigma}=1_{\mathrm{T}_{\mathrm{A}}^{*}} \quad \text { e } \quad \mathrm{E}_{\sigma}^{-1} \mathrm{E}_{\sigma}=1_{\mathrm{T}_{\mathrm{A}}^{*}} .
\end{aligned}
$$

Além disso, para cada $\mathrm{s}$ em $\mathrm{T}_{A}^{*}$ e $\sigma$ em $\mathrm{T}_{\mathrm{A}}$, se $\mathrm{s}$ come ça com $\sigma$ (termina com $\sigma$ ), é fảcil ver que

$$
\mathrm{sD}_{\sigma} \mathrm{D}_{\sigma}^{-1}=\mathrm{s} \quad\left(\mathrm{sE}_{\sigma} \mathrm{E}_{\sigma}^{-1}=\mathrm{s}\right)
$$

A cada aresta $\alpha=\left(q, i_{1}, i_{2}, p\right)$ de $A$ associamos o seu rótulo $|\alpha|$, que é a função parcial

$$
|\alpha|=i_{1} \times i_{2}: \mathrm{T}_{A}^{*} \times \mathrm{T}_{A}^{*} \longrightarrow \mathrm{T}_{A}^{*} \times \mathrm{T}_{A}^{*}
$$


e que pode ser interpretada como um par de instruções, sendo que a primeira (segunda) componente age sobre a fita à esquer da (direita) de $A$; isto é, para cada par $\left(s_{1}, s_{2}\right)$ em $\mathrm{T}_{A}^{*} \times \mathrm{T}_{A}^{*}$,

$$
\left(s_{1}, s_{2}\right)|\alpha|=\left(s_{1} i_{1}, s_{2} i_{2}\right)
$$

se a aresta $\alpha$ for executảvel; caso conträrio, $\left(s_{1}, s_{2}\right)|\alpha|=\emptyset$.

Também podemos associar a $\alpha$ uma função parcial

$$
|| \alpha||: \mathrm{CI}_{\mathrm{A}} \longrightarrow \mathrm{CI}_{\mathrm{A}}
$$

onde $\mathrm{CI}_{A}=\varepsilon \mathrm{T}_{A}^{*} \mathrm{Q}_{A} \mathrm{~T}_{A}^{*} \varepsilon \quad \overrightarrow{\mathrm{e}}$ o conjunto das configurações instantâneas de $A$, de modo que para cada configuração instantânea $u$ de $A, u$ em $C I_{A}, u|| \alpha||$ representa a configurąão instantânea de A após a execução da aresta a. Ou seja, se

$$
u=\varepsilon s_{1} q s_{2} \varepsilon \text { en } C I_{A} \text {, então }
$$

$u|| \alpha||=\left(\varepsilon s_{1} q s_{2} \varepsilon\right)||\left(q, i_{1}, i_{2}, p\right)||=\varepsilon s_{1} i_{1} p s_{2} i_{2} \varepsilon$,

se a aresta $\alpha$ for executáve1; caso contrário, $u|| \alpha||=\emptyset$.

EXEMPI_O 2 - Consideremos a mäquina de Turing A do exemplo 1. Sejam $u_{0}=\varepsilon a q_{0} a b b \varepsilon$ em CI $A$ e as arestas

$\alpha_{1}=\left(q_{0}, E_{a}^{-1}, D_{a}, q_{0}\right)$ e $\alpha_{2}=\left(q_{0}, E_{a}, D_{b}, q_{1}\right)$. Então,

$$
\begin{aligned}
& u_{0}|| \alpha_{1}||=\varepsilon a E_{a}^{-1} q_{0}(a b b) D_{a} \varepsilon=\varepsilon a a_{0} b b \varepsilon=u_{1}, \\
& u_{1}|| \alpha_{1}||=\varepsilon(a a) E_{a}^{-1} q_{0}(b b) D_{a} \varepsilon=\emptyset \\
& u_{1}|| \alpha_{2}||=\varepsilon(a a) E_{a} q_{1}(b b) D_{b} \varepsilon=\varepsilon a q_{1} b \varepsilon .
\end{aligned}
$$


Uma computação de uma máquina de Turing A é realizada por passos. Cada passo consiste na escolha e execução ( se possivel) de uma aresta em $A_{A}$.

Em cada instante, $\odot$ controle finito de $A$ encontra-se num estado $q$ em $Q_{A}$ e com a configuração das fitas $\left(s_{1}, s_{2}\right)$, $\mathrm{s}_{1}$ e $\mathrm{s}_{2} \mathrm{em}_{A}^{*}$. A correspondente configuração instantânea de $A$ é $\varepsilon s_{1} q s_{2} \varepsilon$.

Um passo da computação começa selecionando uma aresta $\alpha$ com origem em $q$, por exemplo $\alpha=\left(q, i_{1}, i_{2}, p\right)$, que poderá ser executada somente se cada instrução $i_{j}$ puder ser executada sobre cada palavra $s_{j}(j=1$ e 2); caso seja, o controle finito transfere para o estado $p$ e a nova configuração das fitas será $\left(t_{1}, t_{2}\right)$, onde cada palavra $t_{j} \bar{e}$ obtida de $s_{j}$ pela execução da instrução $i_{j}(j=1$ e 2$)$.

A correspondente configuração instantânea de A após executar a aresta $\alpha \bar{e} \varepsilon t_{1} p t_{2} \varepsilon$. Caso a aresta $\alpha$ não seja executảvel, esta computação é suspensa e se não existir uma aresta em $A_{A}$, com origem em $q$, que seja executáve1, a máquina pára.

Um passeio não degenerado c numa mäquina de Turing è uma sequência finita de arestas

$$
c=\alpha_{1} \alpha_{2} \cdots \alpha_{k} \quad(k \geq 1)
$$

onde para cada $j \quad(1 \leq j<k)$, o término de $\alpha_{j}$ é igual a origem de $\alpha_{j+1}$. A origem de c é a origem de $\alpha_{1}$ e o término de c é o término de $\alpha_{\mathrm{k}}$. O natural $\mathrm{k}$ é chamado de comprimento do 
passeio c.

Ao passeio c também podemos associar um rótulo $|\mathrm{c}|$, que é a composição dos rótulos das arestas $\alpha_{1}, \alpha_{2}, \ldots, \alpha_{k}$, isto é,

$$
|c|=\left|\alpha_{1}\right|\left|\alpha_{2}\right| \cdots\left|\alpha_{k}\right|
$$

Vamos denotar um passejo c com origem em q e tërmino em p por

$$
c: q \longrightarrow p
$$

A função configuração instantânea de $A$ após a execução de uma aresta $\alpha$ em $\mathrm{A}_{\mathrm{A}}$ também pode ser estendida para o pas seio $c$ em $A$, como segue.

$$
|| c||=|| \alpha_{1}|||| \alpha_{2}|| \ldots|| \alpha_{k}||
$$

Assim para cada $u$ em $\mathrm{CI}_{A}, u|| c||$ representa a configuração instantânea de A após a execução do passeio c.

Definimos também, para cada estado $q$ em $Q_{A}, o$ passeio degenerado ou trivial $1_{q}$, que não contẻm arestas e tem origem e término em q. O comprimento desse passeio é zero e seu rótulo $\left|1_{\mathrm{q}}\right|$ è a função $1_{\mathrm{T}_{\AA}^{*}} \times 1_{\mathrm{T}_{A}^{*}}$ ou $\mathrm{N} \times \mathrm{N}$. Nesse caso, $\| 1_{q}||={ }^{1} I_{A}$, isto é, a função identidade sobre $\mathrm{CI}_{A}$.

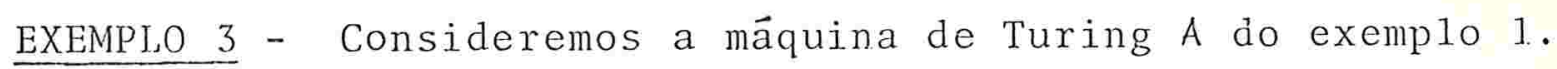


Sejam $u=\varepsilon q_{0}$ abe em $\mathrm{CI}_{A}$ e o passeio $c=\alpha_{1} \alpha_{2}{ }^{\alpha} 3$, com $\alpha_{1}=\left(q_{0}, E_{a}^{-1}, D_{a}, q_{0}\right), \alpha_{2}=\left(q_{0}, E_{a}, D_{b}, q_{1}\right)$ e $\alpha_{3}=\left(q_{1}, v, v, q_{2}\right)$. Então, temos que

$$
\begin{aligned}
(1, \mathrm{ab})|\mathrm{c}| & =(1, \mathrm{ab})\left|\alpha_{1}\right|\left|\alpha_{2}\right|\left|\alpha_{3}\right|=(\mathrm{a}, \mathrm{b})\left|\alpha_{2}\right|\left|\alpha_{3}\right| \\
& =(1,1)\left|\alpha_{3}\right|=(1,1) \\
\left(\varepsilon \mathrm{q}_{0} \mathrm{ab} \varepsilon\right)|| c|| & =\left(\varepsilon q_{0} \mathrm{ab} \varepsilon\right)|| \alpha_{1}|||| \alpha_{2}|||| \alpha_{3}||=\left(\varepsilon \mathrm{aq}_{0} \mathrm{~b} \varepsilon\right)|| \alpha_{2}|||| \alpha_{3}|| \\
& =\left(\varepsilon \mathrm{q}_{1} \varepsilon\right)|| \alpha_{3}||=\varepsilon q_{2} \varepsilon
\end{aligned}
$$

Dados dois passeios $c=\alpha_{1} \alpha_{2} \cdots \alpha_{k}(k \geq 0) \quad e$ $\mathrm{d}=\beta_{1} \beta_{2} \ldots \beta_{\ell}(\ell \geq 0)$ em $A$, tal que a origem de $\mathrm{d}$ é igual ao término de c, definimos o produto cd dos passeios c e d por concatenação, isto é,

$$
c d=\alpha_{1} \alpha_{2} \cdots \alpha_{k} \beta_{1} \beta_{2} \cdots \beta_{\ell} .
$$

E claro que o produto de passeios ê uma operação associativa e que,para todo passeio c com origem em q e término em $\mathrm{p}$,

$$
1_{q} c=c=c 1_{p} \text {. }
$$

Seja s uma palavra em $\Sigma_{\AA}^{*}$. Dizemos que a máquina de Turing A reconhece s se existir um passeio c em A, com origem $q_{-}$em $I_{A}$ e têrmino $q_{+} e m F_{A}$, tal que

$$
(1, s)|c| \in \mathrm{T}_{A}^{*} \times 1
$$


ou, de modo equivalente,

$$
\left(\varepsilon q_{-} s \varepsilon\right)|| c||=\varepsilon t q_{+} \varepsilon \text {, para algum } t \text { em } \mathrm{T}_{A}^{*} \text {. }
$$

Ou seja, dizemos que a palavra $s$ è reconhecida por A se existir um passeio c em A, com origem num estado inicial q_, tal que iniciando a mäquina de Turing A no estado q_e com a configuração das fitas $(1, s)$, cada aresta de c é executável e a execução do passeio c transfere A para algum estado final $q_{+}$ e com a configuração das fitas $(t, 1)$, para algum $t$ em $\mathrm{T}_{A}^{*}$.

O conjunto das palavras reconhecidas por A determi na a linguagem reconhecida por $A$, que denotamos por $|A|$. Assim,

$$
\begin{gathered}
|A|=\left\{s \in \sum_{A}^{*} / \text { existe } c: q \longrightarrow p, \text { com } q \in I_{A} \text { e } p \in F_{A}\right. \text { tal que } \\
\left.(1, s)|c| \in T_{A}^{*} \times 1\right\}
\end{gathered}
$$

ou, de modo equivalente,

$$
|A|=\left\{s \in \Sigma_{A}^{*} / \text { existe } c: q \longrightarrow p, \operatorname{com} q \in I_{A} \text { e } p \in F_{A}\right. \text { tal que }
$$$$
\left.(\varepsilon q s \varepsilon)|| c||=\varepsilon t p \varepsilon, \text { para algum } t \text { em } \mathrm{T}_{A}^{*}\right\} .
$$

No caso do exemplo 1 , temos que $|A|=\left\{a^{n} b^{n} / n \geq 0\right\}$.

Dizemos que duas máquinas de Turing A e B são equi valentes se $|A|=|B|$.

Passamos a definir agora alguns tipos especiais de 
mäquinas de Turing.

Dizemos que uma mäquina de Turing A $\vec{e}$ deterministica se ela tiver no máximo um estado inicial e, para cada es tado $q$ em $Q_{A}$, os domínios dos rótulos das arestas com origem em q forem dois a dois disjuntos.

Uma máquina de Turing A è semi-normalizada se o rótulo de cada aresta em $A_{A}$ tiver pelo menos uma das componentes igual a $N$. Se, além de $A$ ser semj-normalizada, tiver $\left|I_{A}\right|=\left|F_{A}\right|=1$ e o estado inicial (final) não for término (origem) de nenhuma aresta em $A_{A}$, dizemos que A ê normalizada.

Agora, verificamos, para cada estado q de uma máquina de Turing determinística, como são os rótulos das arestas com origem em $q$.

PROPOSIÇÃO 1 - Seja A uma máquina de Turing deterministica. Então, para cada estado $q$ em $Q_{A}$ ou existe no mäximo uma aresta com origem em q ou todas as primeiras (segundas) componentes dos rótulos das arestas com origem em q pertencem a $\left\{\mathrm{E}_{\sigma} / \sigma \in \mathrm{T}_{\mathrm{A}}\right\} \quad U\{\mathrm{~V}\} \quad\left(\left\{\mathrm{D}_{\sigma} / \sigma \in \mathrm{T}_{A}\right\} U\{\mathrm{~V}\}\right)$.

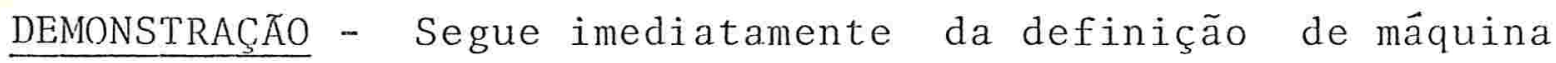
de Turing deterministica e do fato que se X e Y são dois con juntos não vazios então $X \cap \mathrm{T}_{A}^{*}$ e $\mathrm{T}_{A}^{*} \cap \mathrm{Y}$ não são disjuntos. 
Como uma consequência direta dessa proposição temos:

COROLÁRIO 1 - Seja A uma mäquina de Turing determinística e semi-norma1izada. Então, para cada $\mathrm{q}$ em $\mathrm{Q}_{A}$, existe uma compo mente em que os rótulos de todas as arestas com origem em q apresentam $\mathrm{N}$ nessa componente.

\section{MĀQUINA DE TURING REVERSTVEL}

As máquinas de Turing reversíveis, que foram intro duzidas por Lecerf (1963a), constituem uma das ferramentas bạ sicas na demonstração dos dois resultados principais deste trabalho.

Seja A uma mäquina de Turing determinística.

Seja $A^{\prime}$ a mäquina de Turing com

$$
\begin{aligned}
T_{A^{\prime}} & =T_{A}, \\
\Sigma_{A^{\prime}} & =\Sigma_{A^{\prime}}, \\
Q_{A^{\prime}} & =\left\{q^{\prime} / q \in Q_{A^{\prime}}\right\}, \\
I_{A^{\prime}} & =\left\{q^{\prime} / q \in F_{A}\right\}, \\
F_{A^{\prime}} & =\left\{q_{0}^{\prime}\right\}, \text { onde }\left\{q_{0}\right\}=I_{A} \\
\text { e } A_{A^{\prime}} & =\left\{\left(p^{\prime}, i_{1}^{-1}, j_{2}^{-1}, q^{\prime}\right) /\left(q, i_{1}, i_{2}, p\right) \in A_{A^{\prime}}\right\} .
\end{aligned}
$$

Chamamos a máquina de Turing $A^{\prime}$ de reversa da má- 
quina de Turing $A$ e dizemos que $A$ é reversivel se A e A' forem ambas determinísticas.

$$
\text { Denominamos a aresta } \alpha^{\prime}=\left(p^{\prime}, i_{1}^{-1}, i_{2}^{-1}, q^{\prime}\right) \text { em } A_{A^{\prime}}
$$

de reversa da aresta $\alpha=\left(q, i_{1}, i_{2}, p\right)$ em $A_{A}$ e se c é um passeio em $A, c=\alpha_{1} \alpha_{2} \cdots \alpha_{k}, \operatorname{com} k \geq 1$ e $\alpha_{i} \in A_{A}(1 \leq i \leq k)$, dizemos que o passeio $c^{\prime}=\alpha_{k_{k}-1}^{\prime} \alpha_{1}^{\prime} \alpha_{i}^{\prime}$ em $A^{\prime}$ é o reverso do passeio c. Se $c=1_{q}$, para algum $q$ em $Q_{A}$, então $c^{\prime}=1_{q^{\prime}}$.

EXEMPLO 4 - Seja a máquina de Turing determinística

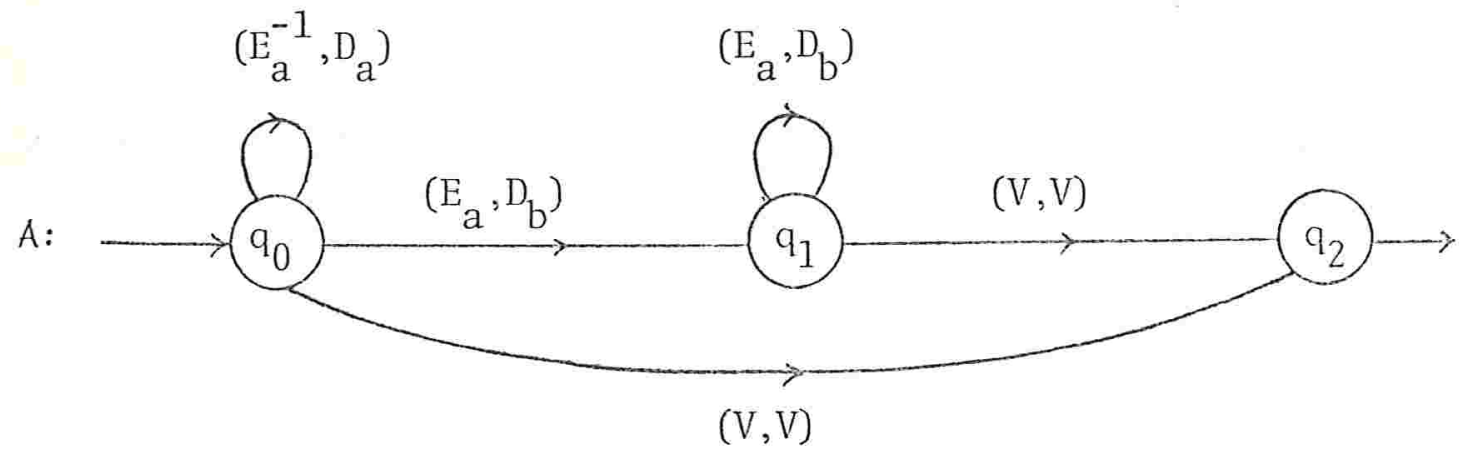

Então, a reversa de A é a máquina de Turing

$$
\left(\mathrm{E}_{\mathrm{a}}, \mathrm{D}_{\mathrm{a}}^{-1}\right) \quad\left(\mathrm{E}_{\mathrm{a}}^{-1}, \mathrm{D}_{\mathrm{b}}^{-1}\right)
$$

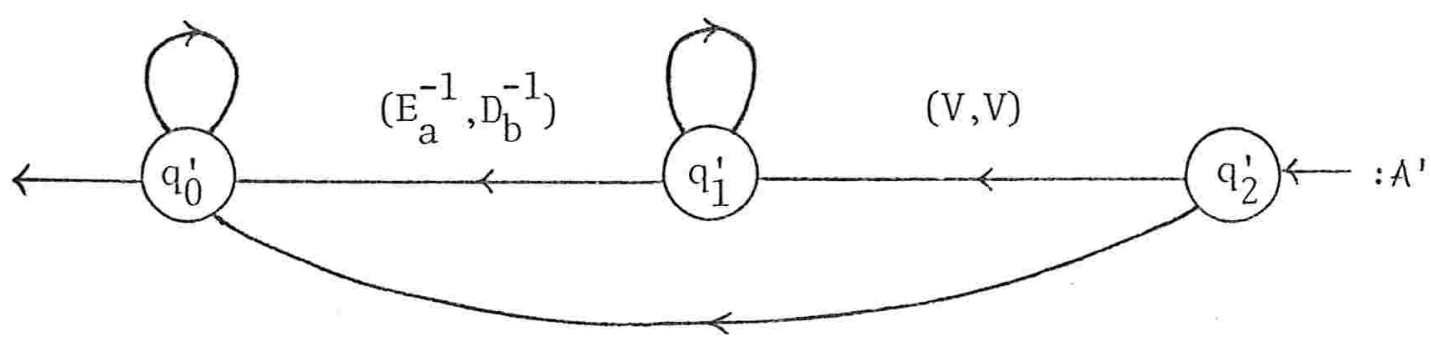

$(\mathrm{V}, \mathrm{V})$ 
Neste caso, como $A^{\prime}$ não é determinística, resulta que A não è reversíve1.

No Exemp1o 6, no final da seção 4 deste capítulo, apresentamos uma máquina de Turing reversível e normalizada, que è equivalente a $A$.

Examinamos então a relação existente entre os passejos numa mäquina de Turing reversível A e os passeios na sua máquina reversa $A^{\prime}$.

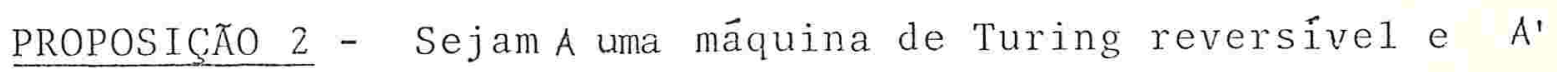
a sua reversa. Para cada passeio $c: q \longrightarrow p$ em $A$ e palavras s e $t$ em $\mathrm{T}_{A}^{*}$, se $(\varepsilon s q t \varepsilon)|| c||=\varepsilon u p v \varepsilon$, para u e $v$ em $\mathrm{T}_{A}^{*}$ então, sendo $c^{\prime}: p^{\prime} \rightarrow q^{\prime}$ o reverso de $c$ em $A^{\prime}$, resulta que $\left(\varepsilon \operatorname{cup}^{\prime} v \varepsilon\right)|| c^{\prime}||=\varepsilon s q^{\prime} t \varepsilon$.

DEMONSTRACÃO - Seja A uma mäquina de Turing reversível e seja $A^{\prime}$ a reversa de $A$.

Sejam c:q $\longrightarrow$ p um passeio em A e palavras $s$ e $t$ em $\mathrm{T}_{A}^{*}$ tais que

$$
(\varepsilon \mathrm{sqt} \varepsilon)|| \mathrm{c}||=\varepsilon u p v \varepsilon \text {, para u e v em } \mathrm{T}_{A}^{*} \text {. }
$$

Demonstramos, por indução no comprimento do passeio c, que se $c^{\prime}: p^{\prime} \longrightarrow q^{\prime}$ ê o reverso de $c$ em $A^{\prime}$, então

$$
\left(\varepsilon u p^{\prime} v \varepsilon\right)|| c^{\prime}||=\varepsilon s q^{\prime} t \varepsilon
$$


Se c é um passeio degenerado, então $c=1_{q}$ e $p=q$. Nesse caso, segue de (1) que $u=s$ e $v=t$. Assim, o passeio $c^{\prime}=1_{p^{\prime}}$, onde $p^{\prime}=q^{\prime}, \dot{e}$ o reverso de $c$ em $A^{\prime} e$

$$
\left(\varepsilon u p^{\prime} v \varepsilon\right)|| c^{\prime}||=-\varepsilon u p^{\prime} v \varepsilon=\varepsilon q^{\prime} t \varepsilon \text {. }
$$

Se c $\in A_{A}$, então $c=(q, i, j, p)$, para algum $i$ em $\mathrm{E}_{\mathrm{A}}$ e $\mathrm{j}$ em $\mathrm{D}_{\mathrm{A}}$. Logo, segue de (1) que

$$
u=s i \quad \text { e } \quad v=t j \text {. }
$$

Portanto, a aresta $c^{\prime}=\left(p^{\prime}, i^{-1}, j^{-1}, q^{\prime}\right)$ em $A_{A}{ }^{\prime} \bar{e} a$ reversa de c e

$$
\left(\varepsilon u p^{\prime} v \varepsilon\right)|| c^{\prime}||=\varepsilon u i^{-1} q^{\prime} v j_{\varepsilon}^{-1}=\varepsilon s i i^{-1} q^{\prime} t j j^{-1} \varepsilon=\varepsilon s q^{\prime} t \varepsilon \text {. }
$$

Suponhamos então que a proposição seja vãlida para passeios em $A$ de comprimento menor do que $k$, para $k \geq 1$ e palavras quaisquer w e $z$ em $\mathrm{T}_{A}^{*}$; vamos prová-la para um passeio c de comprimento $k$ e palavras $s$ e $t$ em $\mathrm{T}_{A}^{*}$.

Seja o passeio $\mathrm{c}: \mathrm{q} \longrightarrow \mathrm{p}, \mathrm{c}=\alpha_{1} \alpha_{2} \ldots \alpha_{\mathrm{k}}$, com $\alpha_{\mathrm{i}}$ em $A_{A}(1 \leq i \leq k)$ e sejam s e $t$ em $T_{A}^{*}$ tal que a condição (1) seja vâlida. Sendo $r$ em $Q_{A}$ a origem de $\alpha_{k}$, temos que o passeio $\mathrm{d}=\alpha_{1} \ldots \alpha_{\mathrm{k}-1}$ tem origem em q e término em $\mathrm{r}$. Logo, existem palavras $x$ e y em $\mathrm{T}_{A}^{*}$ tais que.

$$
(\varepsilon \mathrm{sqt} \varepsilon)|| \mathrm{d}||=\operatorname{exry\varepsilon } \quad \dot{\mathrm{e}}(\operatorname{exry\varepsilon })|| \alpha_{\mathrm{k}}||=\varepsilon \text { upve. }
$$

E pela hipótese da indução, aplicada ao passeio d, segue que o passeio $d^{\prime}: r^{\prime} \longrightarrow q^{\prime}$ è o reverso de d em $A^{\prime}$ e 


$$
\left(\varepsilon \times r^{\prime} y \varepsilon\right)|| d^{\prime}||=\varepsilon s q^{\prime} t \varepsilon
$$

Alëm disso, a aresta $\alpha_{k}^{\prime}: p^{\prime} \longrightarrow r^{\prime}$, reversa de $\alpha_{k}$, pertence a $A_{A^{\prime}} \quad e$

$$
\left(\varepsilon \operatorname{up}^{\prime} v \varepsilon\right)|| \alpha_{k}^{\prime}||=\varepsilon x r^{\prime} y \varepsilon
$$

Mas, $c^{\prime}=\alpha_{k}^{\prime} d^{\prime} \quad$ è um passeio em $A^{\prime}$ com origem em p' e término em $q^{\prime}$, onde $d^{\prime}=\alpha_{k-1}^{\prime} \cdots \alpha_{1}^{\prime}$. Então, $c^{\prime}=\alpha_{k}^{\prime} \alpha_{k-1}^{\prime} \cdots \alpha_{1}^{\prime} \vec{e} \quad$ o reverso de c em $A^{\prime}$ e

$$
\text { ( } \left.\operatorname{up}{ }^{\prime} v \varepsilon\right)|| c^{\prime}||=\left(\varepsilon u p^{\prime} v \varepsilon\right)|| \alpha_{k}^{\prime}|||| d^{\prime}||=\left(\varepsilon x r^{\prime} y \varepsilon\right)|| d^{\prime}||=\varepsilon s q^{\prime} t \varepsilon \text {. }
$$

Desse modo, temos demonstrado a Proposição 2.

EXEMPLO 5 - Sejam as mäquinas de Turing $A$ e $A^{\prime}$ como no Exemplo 4 e consideremos a configuração instantânea $\varepsilon_{\mathrm{a}_{0}}$ abe de $A$ e $O$ passeio $c=\alpha_{1} \alpha_{2} \alpha_{3}$ em $A$, com $\alpha_{1}=\left(q_{0}, E_{a}^{-1}, D_{a}, q_{0}\right), \alpha_{2}=\left(q_{0}, E_{a}, D_{b}, q_{1}\right) \quad$ e $\alpha_{3}=\left(q_{1}, v, v, q_{2}\right)$ tais que

$$
\left(\varepsilon q_{0} a b \varepsilon\right)|| c||=\varepsilon q_{2} \varepsilon \text {. }
$$

Seja c'em $A^{\prime}$, o passeio reverso de c. Então, $c^{\prime}=\alpha_{3}^{\prime} \alpha_{2}^{\prime} \alpha_{1}^{\prime}, \quad$ com $\alpha_{3}^{\prime}=\left(q_{2}^{\prime}, v, v, q_{1}^{\prime}\right), \alpha_{2}^{\prime}=\left(q_{1}^{\prime}, E_{a}^{-1}, D_{b}^{-1}, q_{0}^{\prime}\right) \quad$ e $\alpha_{1}^{\prime}=\left(q_{0}^{\prime}, E_{a}, D_{a}^{-1}, q_{0}^{\prime}\right)$. Assim,

$$
\begin{aligned}
\left(\varepsilon \mathrm{q}_{2}^{\prime} \varepsilon\right)|| c^{\prime}|| & =\left(\varepsilon \mathrm{q}_{2}^{\prime} \varepsilon\right)|| \alpha_{3}^{\prime}|||| \alpha_{2}^{\prime}|||| \alpha_{1}^{\prime}|| \\
& =\left(\varepsilon \mathrm{q}_{1}^{\prime} \varepsilon\right)|| \alpha_{2}^{\prime}|||| \alpha_{1}^{\prime} \| \\
& =\left(\varepsilon \mathrm{aq}_{0}^{\prime} \mathrm{b} \varepsilon\right)|| \alpha_{1}^{\prime}|| \\
& =\varepsilon \mathrm{q}_{0}^{\prime} \mathrm{ab} \varepsilon
\end{aligned}
$$


4. SIMULACAO DE UMA MĀQUINA DE TURING DETERMINISTICA POR UMA REVERSIVEL E NORMALIZADA

Enunciamos agora o resultado principal deste capítulo que assegura que cada 1 inguagem recursivamente enumeráve1 é o comportamento de alguma máquina de Turing reversível e normalizada.

TEOREMA 1 - Cada mäquina de Turing determinística é equivalente a alguma mäquina de Turing reversível e normalizada.

Com a finalidade de demonstrá-1o, apresentamos dois lemas.

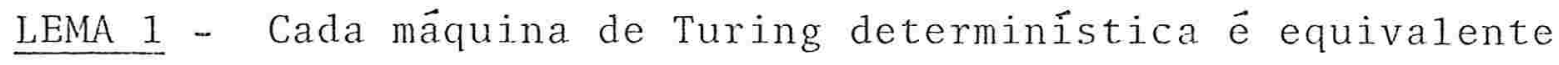
a alguma máquina de Turing determinística e semi-normalizada.

DEMONSTRAÇÃO - Seja A uma mäquina de Turing determinística. Construímos uma máquina de Turing B com

$$
\begin{aligned}
& \mathrm{T}_{B}=\mathrm{T}_{A}, \\
& \Sigma_{B}=\Sigma_{A}, \\
& \mathrm{Q}_{B}=\mathrm{Q}_{A} \cup \hat{\mathrm{Q}}_{1} \cup \mathrm{Q}_{2} \times\left(\mathrm{T}_{B} \cup\{\varepsilon\}\right),
\end{aligned}
$$

onde $\quad \hat{Q}_{1}=\left\{\hat{q} / q \in Q_{A}\right.$ e $\left.\left|A_{A}(q)\right|=1\right\}$

$\mathrm{e}$

$$
Q_{2}=\left\{q \in Q_{A} /\left|A_{A}(q)\right|>1\right\} ;
$$




$$
\begin{aligned}
& \mathrm{I}_{B}=\mathrm{I}_{A}, \\
& \mathrm{~F}_{B}=\mathrm{F}_{A}
\end{aligned}
$$

e $\quad A_{B}$ é constituído por:

- para cada $q$ em $Q_{A}$, com $\left|A_{A}(q)\right|=1$, seja $\alpha=(q, i, j, p)$ em $A_{A}(q)$. Então, as arestas

$$
\beta_{1}=(q, i, N, \hat{q}) \text { e } \beta_{2}=(\hat{q}, N, j, p)
$$

pertencem a $A_{B}$ e é claro que

$$
|\alpha|=\left|\beta_{1}\right|\left|\beta_{2}\right| \text {. }
$$

Graficamente, se em A ocorrer

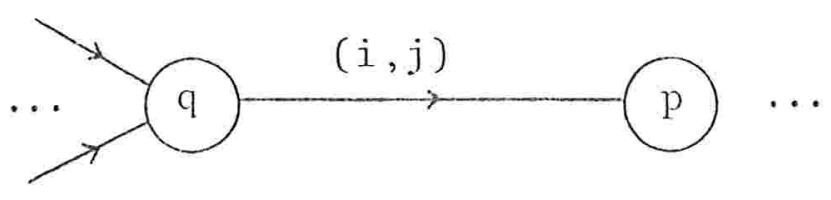

então B terá

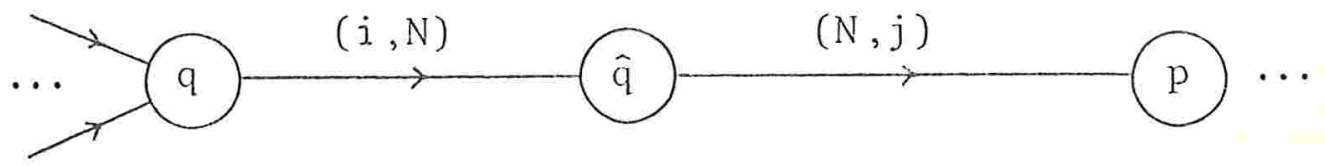

- para cada $q$ em $Q_{A}$, com $\left|A_{A}(q)\right|>1$, resulta pela Proposição 1 que ou todas as arestas em $A_{A}(q)$ têm as primeiras componen 
tes de seus rótulos em $\left\{\mathrm{E}_{\sigma} / \sigma \in \mathrm{T}_{\mathrm{A}}\right\} \mathrm{U}\{\mathrm{V}\}$ ou todas elas têm as segundas componentes de seus rótulos em $\left\{D_{\sigma} / \sigma \in \mathrm{T}_{A}\right\} \cup\{V\}$.

Consideremos, então, os dois casos separadamente.

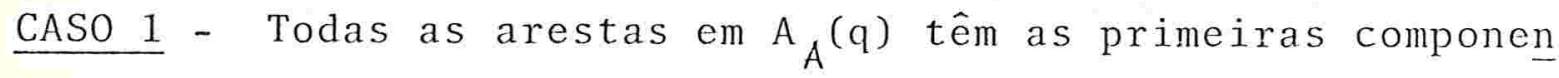
tes de seus rótulos em $\left\{\mathrm{E}_{\sigma} / \sigma \in \mathrm{T}_{A}\right\} U\{\mathrm{~V}\}$.

Então, para cada aresta $\alpha=(q, i, j, p)$ em $A_{A}(q)$, sendo $\gamma=\varepsilon$ se $i=V$ ou $\gamma=\sigma$ em $T_{A}$ se $i=E_{\sigma}$, as arestas

$$
\beta_{1}=(q, i, N,(q, \gamma)) \text { e } B_{2}=((q, \gamma), N, j, p)
$$

pertencem a $A_{B}$. Novamente, è claro que

$$
|\alpha|=\left|\beta_{1}\right|\left|\beta_{2}\right|
$$

Graficamente, se a mäquina de Turing A apresentar o trecho abaixo, com $\left|A_{A}(q)\right|=k$.

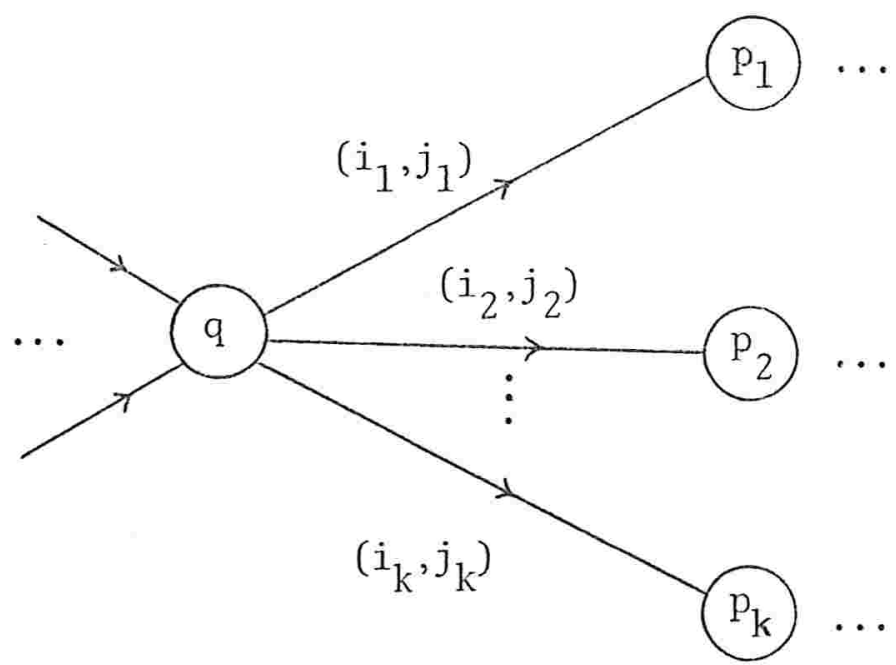


então, o correspondente trecho em B serâ

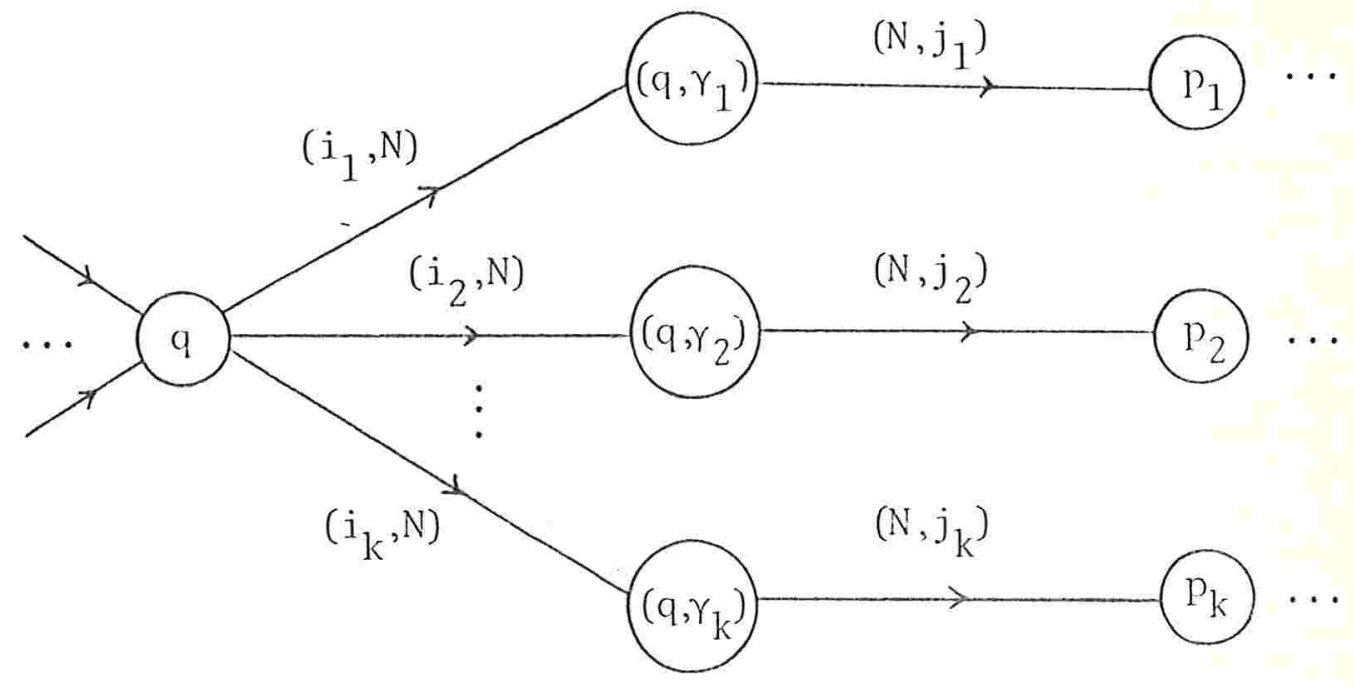

onde, para cada $\ell, 1 \leq \ell \leq k, \gamma_{\ell}=\sigma$ se $i_{\ell}=E_{\sigma}$ para $\sigma \mathrm{em}$ $\mathrm{T}_{\mathrm{A}}$ ou $\gamma_{\ell}=\varepsilon$ se $i_{\ell}=V$.

CASO 2 - Todas as arestas em $A_{A}(q)$ têm as segundas componentes de seus rótulos em $\left\{D_{\sigma} / \sigma \in T_{A}\right\} \cup\{V\}$. Então, para cada aresta $\alpha=(q, i, j, p)$ em $A_{A}(q)$, sendo $\gamma=\varepsilon$ se $j=V$ ou $\gamma=\sigma \mathrm{em}$ $\mathrm{T}_{\mathrm{A}}$ se $\mathrm{j}=\mathrm{D}_{\sigma}$, as arestas

$$
\beta_{1}=(q, N, j,(q, \gamma)) \text { e } \beta_{2}=((q, \gamma), i, N, p)
$$

pertencem a $A_{B}$. Também é claro que

$$
|\alpha|=\left|\beta_{1}\right|\left|\beta_{2}\right| \text {. }
$$

Para vermos graficamente o que acontece, considere mos o seguinte trecho na máquina de Turing $A, \operatorname{com}\left|A_{A}(q)\right|=k$. 


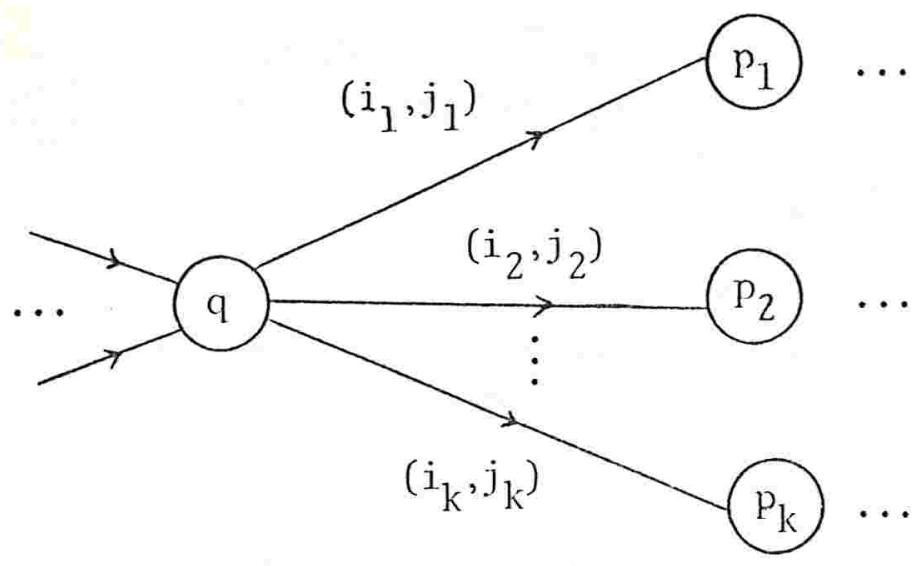

Então, o trecho correspondente em B será

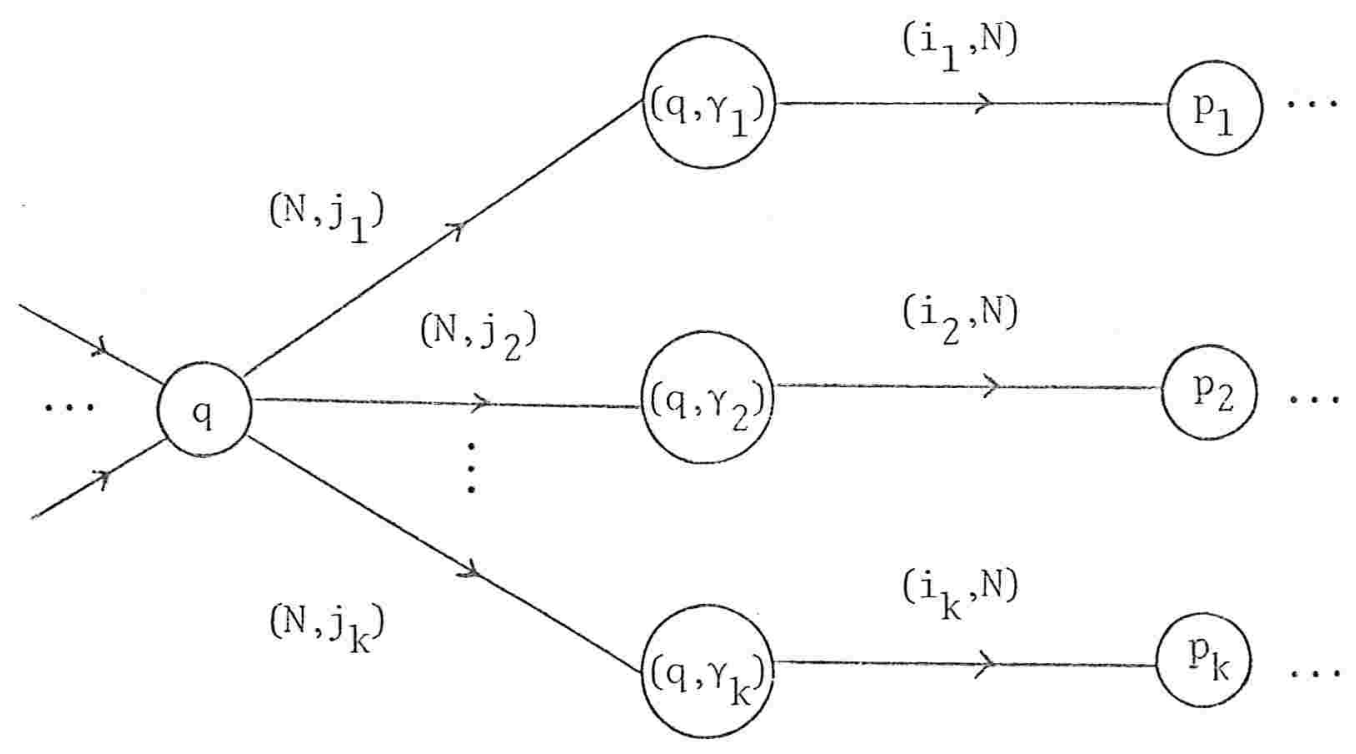

onde, para cada $\ell, 1 \leq \ell \leq k, \gamma_{\ell}=\varepsilon$ se $j_{\ell}=V$ ou $\gamma_{\ell}=\sigma$ se $\mathrm{j}_{\ell}=\mathrm{D}_{\sigma}$ para $\sigma$ em $\mathrm{T}_{A}$.

Assim, de (2), (3) e (4) resulta que $|A|=|B|$ e podemos verificar pelas arestas em $A_{B}$ que $B$ é determinística e semi-normalizada. Finalizamos, portanto, a demonstração do Lema 1 . 
LEMA 2 - Cada máquina de Turing determinística é equivalente a alguma māquina de Turing determinística e normalizada.

DEMONSTRAÇÃO - Seja A uma mäquina de Turing determinística . Pelo Lema 1 existe uma máquina de Turing deterministica e semi-normalizada $C$ tal que $|C|=|A|$.

Definimos, então, uma måquina de Turing B com

$$
\begin{aligned}
& \mathrm{T}_{B}=\mathrm{T}_{C}, \\
& \Sigma_{B}=\Sigma_{C}, \\
& \mathrm{Q}_{B}=Q_{C} \cup\left\{\mathrm{p}_{-}, \mathrm{p}_{+}\right\} \cup \mathrm{Q}_{1} \times \mathrm{T}_{C} \cup \hat{\mathrm{Q}}_{1},
\end{aligned}
$$

onde $\left\{p_{-}, p_{+}\right\} \cap Q_{C}=\emptyset$,

e

$$
\begin{aligned}
\mathrm{Q}_{1}= & \left\{\mathrm{q} \in \mathrm{F}_{C} / \text { existe alguma aresta em } A_{C}\right. \text { com origem } \\
& \text { em } \mathrm{q}\} \\
\hat{\mathrm{Q}}_{1}= & \left\{\hat{\mathrm{q} / q} \in \mathrm{Q}_{1}\right\} ; \\
\mathrm{I}_{B}= & \left\{\mathrm{p}_{-}\right\}, \mathrm{F}_{B}=\left\{\mathrm{p}_{+}\right\}
\end{aligned}
$$

e $\quad A_{B} \quad \bar{e}$ constituído pelas arestas:

$-\left(p_{-}, N, N, q_{-}\right)$, onde $\left\{q_{-}\right\}=I_{C}$

- para cada estado $q$ em $F_{C}$, a aresta $\left(q, N, V, p_{+}\right)$pertence a $A_{B} ;$

- para cada q em $Q_{1}$ e para cada $\sigma$ em ${ }^{T}$, as arestas

$$
\left(q, N, D_{\sigma},(q, \sigma)\right) \text { e }\left((q, \sigma), N, D_{\sigma}^{-1}, \hat{q}\right)
$$


pertencem a $A_{B}$;

- para cada aresta $(q, i, j, p)$ em $A_{C}$, com $q \in Q_{1}$, a aresta

$$
(\hat{q}, i, j, p)
$$

pertence a $A_{B}$;

- cada aresta em $A_{C}$ que não tem origem em $F_{C}$ também pertence a $A_{B}$.

Para um melhor entendimento da construção da máquina de Turing $B$, observemos graficamente o que ocorre.

Consideremos a seguinte máquina de Turing $C$, com $\mathrm{T}_{\mathrm{C}}=\left\{\sigma_{1}, \sigma_{2}, \ldots, \sigma_{n}\right\}$

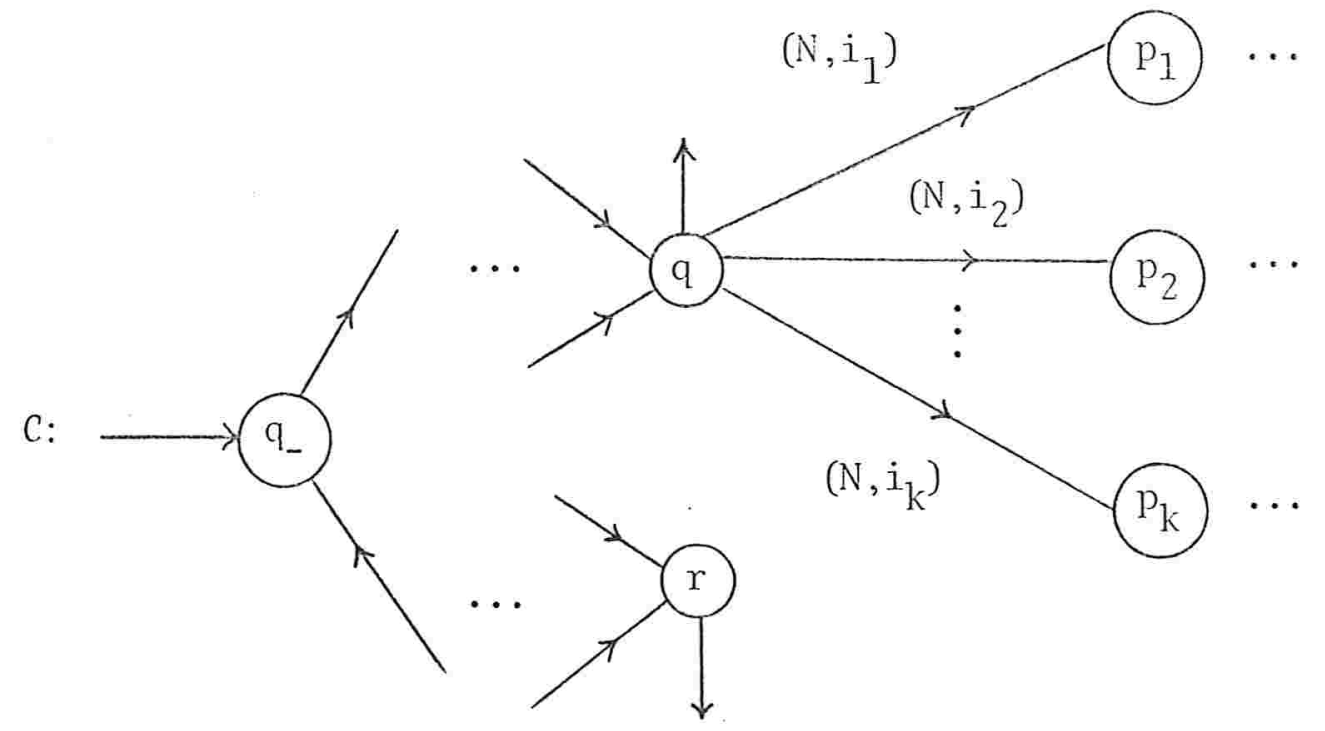


Lembremos que, como $C \vec{e}$ deterministica e semi-normalizada, resulta pelo Corolärio 1 que para cada q cm $Q_{C}$ exis te uma componente (escolhemos a primeira) em que os rótulos de todas as arestas com origem em q apresentam $N$ nessa componente.

Nesse caso, uma mäquina de Turing determinística, normalizada e equivalente a $C$ é dada por:

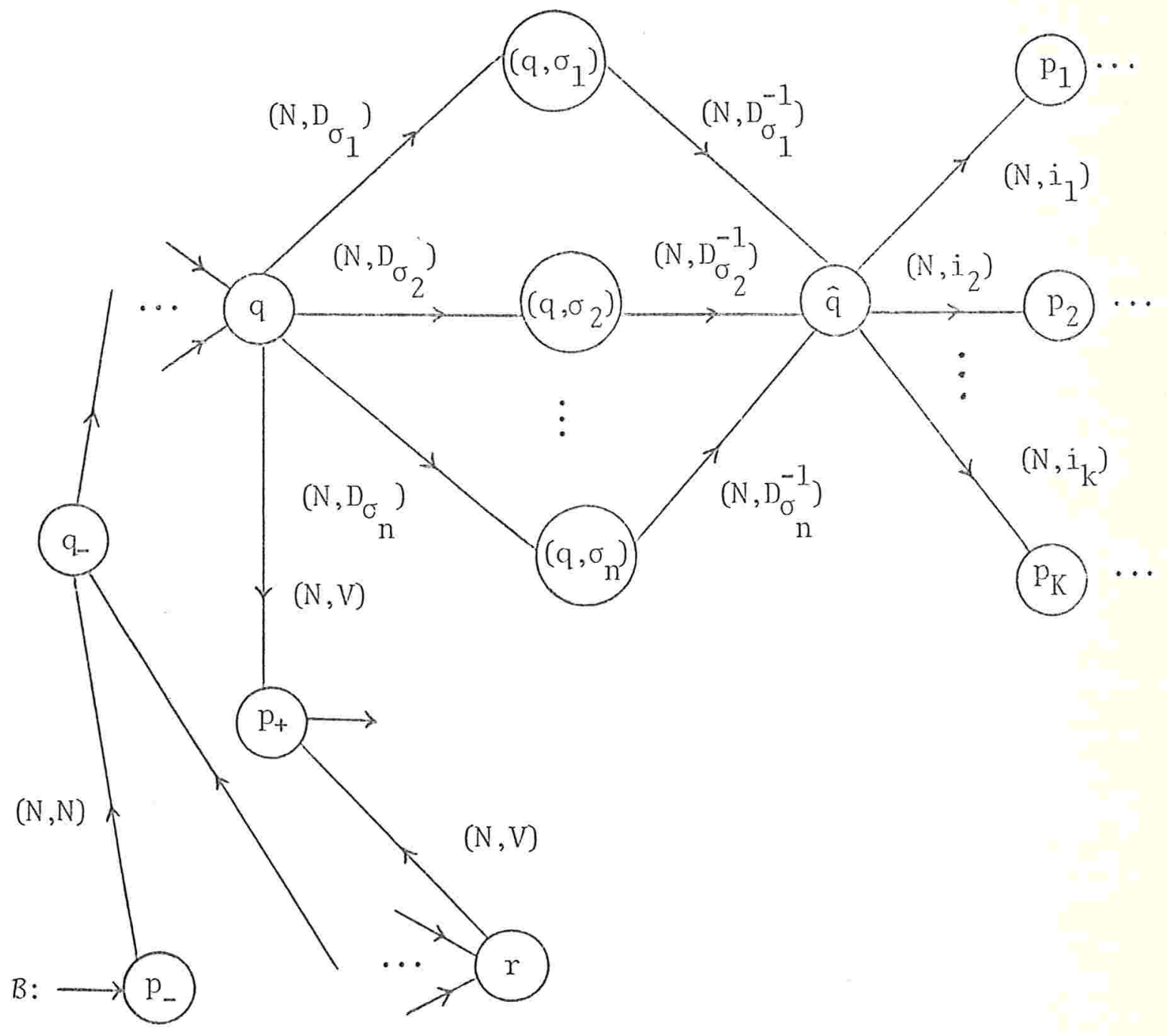


Prosseguindo a demonstração, é fácil ver que $B$ é determinística e normalizada. Mostramos agora que $|B|=|C|$. Seja $s \in|C|$. Então, existe um passeio $c: q_{-} \longrightarrow q_{+}$ em $C$, com $q_{+}$em $F_{C}$ tal que

$$
(1, \mathrm{~s})|\mathrm{c}| \in \mathrm{T}_{\mathrm{C}}^{*} \times 1 .
$$

Se c é um passeio degenerado, então $c=1_{q_{-}}, q_{-}=q_{+}$ está em F $F_{C}$ e $s=1$. Logo,

$$
d=\left(p_{-}, N, N, q_{-}\right)\left(q_{-}, N, V, p_{+}\right)
$$

é um passeio em B de $p_{-}$a $p_{+}$tal que

$$
(1, s)|d| \in T_{B}^{*} \times 1 .
$$

Ou seja, s $\in|B|$.

$$
\begin{aligned}
& \text { Caso contrário, o passeio c tem uma decomposição } \\
& \mathrm{c}=\mathrm{c}_{1} \mathrm{c}_{2} \cdots \mathrm{c}_{\mathrm{k}} \text {, para algum } \mathrm{k}>0 \text {, }
\end{aligned}
$$

tal que $c_{1}$ é o passeio de comprimento minimal com término em $\mathrm{F}_{C}$ e para cada $\ell, 2 \leq \ell \leq k, c_{\ell}$ é um passeio não degenerado minimal com origem e tërmino em $\mathrm{F}_{C}$.

Observemos que no passeio $c_{1}$ não ocorrem arestas com origem em $\mathrm{F}_{C}$. Resulta, então, que $c_{1}$ também é um passeio em $B$.

$$
\begin{aligned}
& \text { Seja } i(1 \leq i \leq k) \text { o menor indice tal que } \\
& (1, s)\left|c_{1} c_{2} \ldots c_{i}\right|=(t, 1) \text {, para algum } t \text { em } T_{C}^{*} .
\end{aligned}
$$


Suponhamos que $i=1$ e seja r o término de $c_{1}$. Como r está em $F_{C}$, a aresta $\left(r, N, V, P_{+}\right)$pertence a $A_{B}$.

$$
\begin{aligned}
& \text { Assim, } B \text { tem o passeio } d: p_{-} \longrightarrow p_{+} \text {, } \\
& \mathrm{d}=\left(\mathrm{p}_{-}, \mathrm{N}, \mathrm{N}, \mathrm{q}_{-}\right) \mathrm{c}_{1}\left(\mathrm{r}, \mathrm{N}, \mathrm{V}, \mathrm{p}_{+}\right) \\
& \text {ta1 que } \quad(1, s)|d|=(1, s)\left|\left(p_{-}, N, N, q_{-}\right)\right|\left|c_{1}\right|\left|\left(r, N, V, p_{+}\right)\right| \\
& =(1, s)\left|c_{1}\right|\left|\left(r, N, V, p_{+}\right)\right| \\
& =(t, 1)\left|\left(r, N, V, p_{+}\right)\right| \\
& =(t, 1) \in T_{B}^{*} \times 1 .
\end{aligned}
$$

Suponhamos agora que $i \geq 2$. De (5) segue que, para cada $j, 2 \leq j \leq i$, existe una aresta $\alpha_{j}=\left(q_{j}, j_{1}, j_{2}, p_{j}\right)$ em $A_{C}$ e um passeio $m_{j}$ em $c$, com origem em $p_{j}$ tal que $c_{j}=\alpha_{j} m_{j}$; alêm disso, $m_{j}$ é um passeio em B, jâa que nele não ocorrem ares tas com origem em Fic.

Logo, correspondendo a cada passeio $c_{j}, 2 \leq j \leq i$, existe algum $\sigma$ em $\mathrm{T}_{B}$ (dependendo de $\mathrm{s}$, ou seja, $\sigma$ è o símbolo na extremidade esquerda da fita à direita quando $C$ for executar a aresta $\alpha_{j}$ ) tal que

$$
\mathrm{d}_{j}=\left(q_{j}, N, D_{\sigma},\left(q_{j}, \sigma\right)\right)\left(\left(q_{j}, \sigma\right), N, D_{\sigma}^{-1}, \hat{q}_{j}\right)\left(\hat{q}_{j}, j_{1}, j_{2}, p_{j}\right) m_{j}
$$

é um passeio em $B$.

Está claro que, para cada configuração das fitas $(x, y)$ de $C$, se $y=\sigma w$, para algum $w$ em $T_{C}^{*}$, então

$$
(x, y)\left|c_{j}\right|=(x, y)\left|d_{j}\right| \text {. }
$$


Assim, existe em $B$ o passeio $d: p_{-} \longrightarrow p_{+}$,

$d=\left(p_{-}, N, N, q_{-}\right) c_{1} d_{2} \cdots d_{i}\left(q, N, V, p_{+}\right)$

onde $q$ é o tërmino de $d_{i}$ e $q \in F_{C}$.

Portanto, utilizando (6) e (7), temos que

$$
\begin{aligned}
(1, \mathrm{~s})|\mathrm{d}| & =(1, \mathrm{~s})\left|\left(\mathrm{p}_{-}, \mathrm{N}, \mathrm{N}, \mathrm{q}_{-}\right)\right|\left|\mathrm{c}_{1}\right|\left|\mathrm{d}_{2}\right| \ldots\left|\mathrm{d}_{\mathrm{i}}\right|\left|\left(\mathrm{q}, \mathrm{N}, \mathrm{V}, \mathrm{p}_{+}\right)\right| \\
& =(1, \mathrm{~s})\left|\mathrm{c}_{1}\right|\left|\mathrm{d}_{2}\right| \ldots\left|\mathrm{d}_{i}\right|\left|\left(\mathrm{q}, \mathrm{N}, \mathrm{V}, \mathrm{p}_{+}\right)\right| \\
& =(t, 1)\left|\left(\mathrm{q}, \mathrm{N}, \mathrm{V}, \mathrm{p}_{+}\right)\right| \\
& =(t, 1) \in \mathrm{T}_{B}^{*} \times 1 .
\end{aligned}
$$

Logo, $s \in|B|$ e, portanto, $|C| \subseteq|B|$. Analogamente, demonstra se que $|B| \subseteq|C|$. E como $|A|=|C|$, concluímos que $|A|=|B|$.

Desse modo, temos provado o Lema 2.

Estamos agora em condições de demonstrar o Teorema 1 que enunciamos novamente a seguir.

TEOREMA 1 - Cada mäquina de Turing deterministica ë equivalen te a alguma máquina de Turing reversível e normalizada.

DEMONSTRACAO - Seja A uma máquina de Turing determinística. Pe1o Lema 2, existe uma máquina de Turing determinística e nor malizada $C$ tal que $|A|=|C|$. 
Definimos então uma mäquina de Turing $B$ com

$$
\mathrm{T}_{B}=\mathrm{T}_{C} \cup\{\$, \xi\} \quad U x
$$

onde $\{\$, \xi\} \cap \mathrm{T}_{\mathrm{C}}=\emptyset$

e $X=\left\{x_{1}, x_{2}, \ldots x_{k}\right\}$ tal que $k=\left|A_{C}\right|$ e $X \cap\left(T_{C} U\{\$, \xi\}\right)=\emptyset$.

Para cada $i, \quad 1 \leq i \leq k$, o i-ésimo símbolo $x_{i}$ está associado à i-ésima aresta de $C$ (supondo-se uma enumeração qualquer das arestas em $\mathrm{A}_{\mathrm{C}}$ ).

Seja $\left(s_{0}, t_{0}\right)$ uma configuração inicial das fitas de $C$, com $s_{0}$ e $t_{0}$ em $\mathrm{T}_{\mathcal{C}}^{*} \cdot \mathrm{A}$ mäquina $B$ será construída de modo que , para cada configuração das fitas $(s, t)$ de $c$, com $s$ e $t$ em $T_{C}^{*}$, atingivel a partir de $\left(s_{0}, t_{0}\right)$, a correspondente configuração das fitas de $B$, partindo de $\left(s_{0}, t_{0}\right)$, será $(s \xi x, t)$, onde $x \in X^{*}$ representa a sequência dos símbolos associados às ares tas utilizadas por $c$ partindo de $\left(s_{0}, t_{0}\right)$ até atingir a configuração $(s, t)$.

$$
\begin{gathered}
\Sigma_{B}=\Sigma_{C}, \\
Q_{B}=Q_{C} \cup\left\{t_{-}\right\} \cup Q_{1} \times H_{1} \cup Q_{2} \times\left(\left\{\sigma_{1}, \sigma_{2}\right\} U x\right) U \\
U Q_{3} \times\left(\left\{\sigma_{3}, \sigma_{4}, \sigma_{5}\right\} \cup x\right) \times H_{3},
\end{gathered}
$$

onde $t_{-} \notin Q_{C}$,

$$
\left\{\sigma_{1}, \sigma_{2}, \sigma_{3}, \sigma_{4}, \sigma_{5}\right\} \cap\left(X \cup \mathrm{T}_{C}\right)=\emptyset
$$

$\mathrm{Q}_{1}=\left\{\mathrm{p} \in \mathrm{Q}_{\mathrm{C}} / \mathrm{C}\right.$ tem alguma aresta com tērmino em $\mathrm{p}$ e rötulo $\mathrm{N} \times \mathrm{i}_{2}$, para algum $\mathrm{i}_{2}$ em $\left.\mathrm{D}_{\mathrm{C}}\right\}$, 
$\mathrm{H}_{1}=\{\mathrm{i} \in \mathrm{IN} / \mathrm{I} \leq \mathrm{i} \leq \mathrm{k}$ e a i-ésima aresta de $\mathrm{c}$ tem rótu$10 \mathrm{~N} \times \mathrm{i}_{2}$, para algum $\left.\mathrm{i}_{2} \mathrm{em}_{\mathrm{C}}\right\}$,

$Q_{2}=\left\{q \in Q_{c} / C\right.$ tem alguma aresta com origem em q e rötulo $i_{1} \times N$, para algum $i_{1}$ em $\left.E_{C}\right\}$,

$\mathrm{Q}_{3}=\left\{\mathrm{p} \in \mathrm{Q}_{\mathrm{C}} / \quad \mathrm{C}\right.$ tem alguma aresta com término em $\mathrm{p}$ e rótu$10 i_{1} \times N$, para algum $i_{1}$ em $\left.E_{C}\right\}$ e

$\mathrm{H}_{3}=\{\mathrm{i} \in \mathbb{I N} / 1 \leq \mathrm{i} \leq \mathrm{k}$ e a $\mathrm{i}$-ésima aresta de $c$ tem rótulo $\mathrm{i}_{1} \times \mathrm{N}$, para algum $\mathrm{i}_{1}$ em $\left.\mathrm{E}_{\mathrm{C}}\right\}$;

$$
\begin{aligned}
& I_{B}=\left\{t_{-}\right\}, \\
& F_{B}=F_{C}
\end{aligned}
$$

e $A_{B}$ consiste

- da aresta $\left(t_{-}, E_{\xi}^{-1}, N, q_{-}\right)$, onde $\left\{q_{-}\right\}=I_{C}$;

- para cada aresta $\alpha$ em $A_{C}$, se $\alpha$ é a i-ésima aresta de $C$ e $\alpha=\left(q, N, i_{2}, p\right), c o m i_{2} \in D_{C}$, então as arestas

$$
\left(q, N, i_{2},(p, i)\right) \quad \text { e }\left((p, i), E_{x_{i}}^{-1}, N, p\right)
$$

pertencem a $A_{B}$.

Graficamente, se

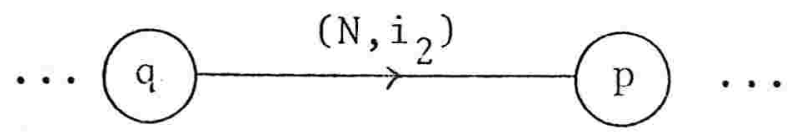

é a i-ēsima aresta de $C$, então em $B$ existirão as arestas 


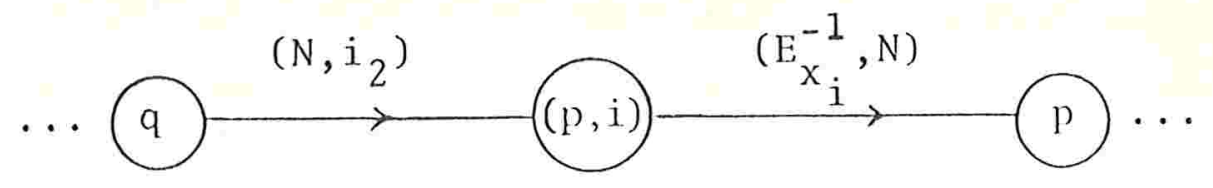

- para cada aresta $\alpha$ em $A_{C}$, se $\alpha$ é a i-ésima aresta de $C$ e $\alpha=\left(q, i_{1}, N, p\right)$, com $i_{1} \in E_{C}$, então as arestas $\left(q, N, D_{\$}^{-1},\left(q, \sigma_{1}\right)\right)$ $\left(\left(q, \sigma_{1}\right), E_{a}, N,(q, a)\right)$ e $\left((q, a), N, D_{a}^{-1},\left(q, \sigma_{1}\right)\right)$, para cada a em $x$, $\left(\left(q, \sigma_{1}\right), E_{\xi}, N,\left(q, \sigma_{2}\right)\right)$ $\left(\left(q, \sigma_{2}\right), i_{1}, N,\left(p, \sigma_{3}, i\right)\right)$, $\left(\left(p, \sigma_{3}, i\right), E_{\xi}^{-1}, N,\left(p, \sigma_{4}, i\right)\right)$ $\left(\left(p, \sigma_{4}, i\right), N, D_{a},(p, a, i)\right)$ e $\left((p, a, i), E_{a}^{-1}, N,\left(p, \sigma_{4}, i\right)\right)$, para cada a em $X$, $\left(\left(p, \sigma_{4}, i\right), N, D_{\$},\left(p, \sigma_{5}, i\right)\right) e$ $\left(\left(p, \sigma_{5}, i\right), E_{x_{i}}^{-1}, N, p\right)$ pertencem a $A_{B}$.

Graficamente, seja o seguinte trecho em $C$,

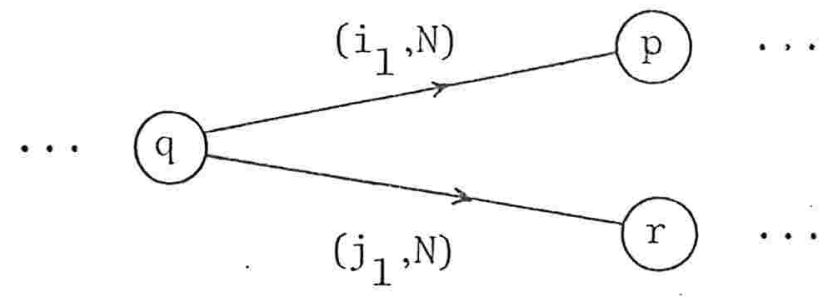

onde $\left(q, i_{1}, N, p\right)$ é a i-ësima aresta de $\mathrm{C}$ e $\quad\left(q, j_{1}, N, r\right)$ è a j-ësima aresta de $C$, para $1 \leq i, j \leq k, i \neq j$ e $i_{1}$ e $j_{1}$ em $E_{C}$. 
Então $B$ terá as arestas que apresentamos na Figura 1, onde utilizamos um $\square$ ao invés de representar todos os $\mathrm{k}$ estados que correspondem a cada um dos $x_{\ell}$ em $x, 1 \leq \ell \leq k$.

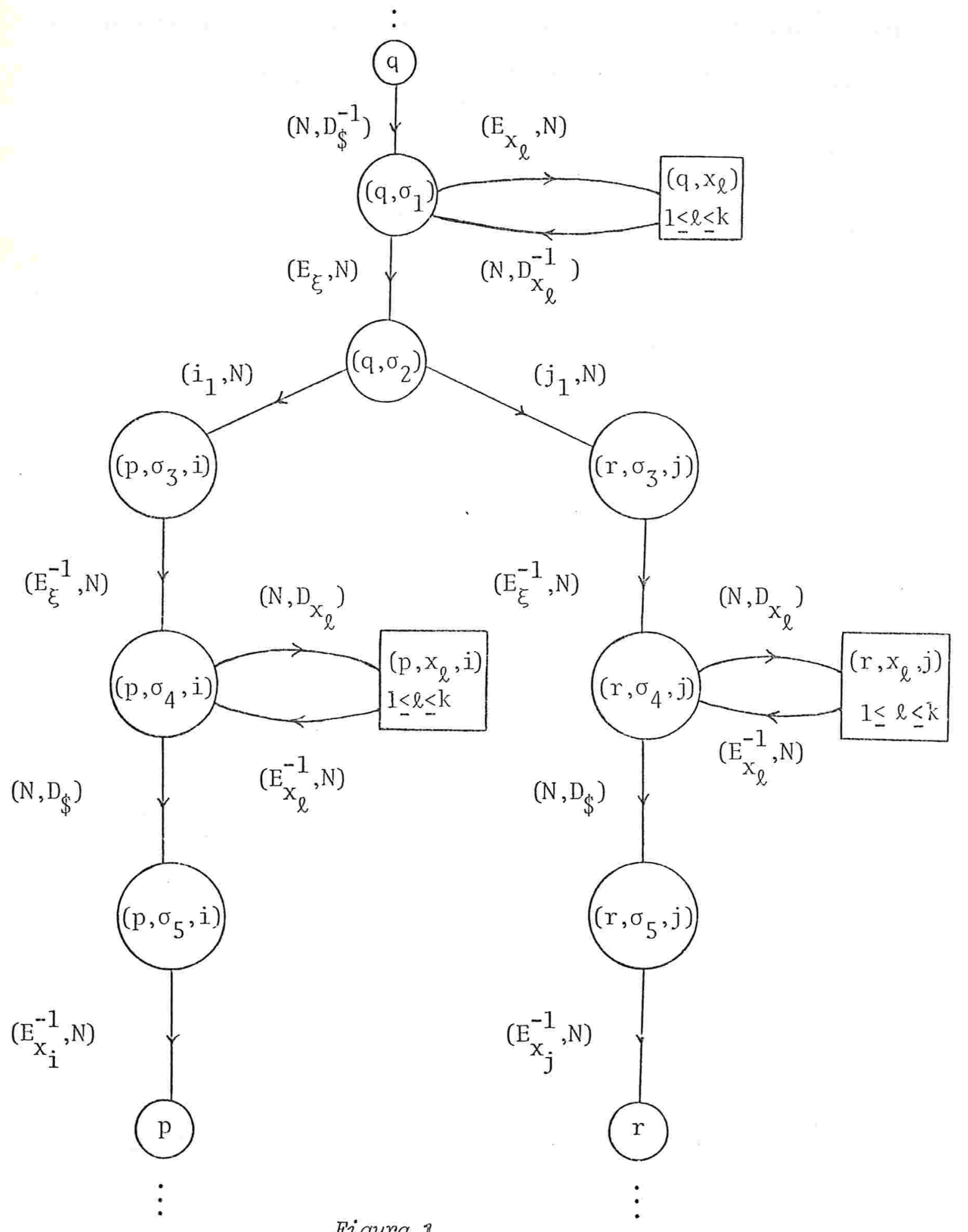

Figura ? 
Pode-se verificar que $B$ é uma mâquina de Turing re versíve1, bastando para isso examinar que $B$ e a sua reversa são ambas determinísticas.

Resta-nos provar que $|A|=|B|$. Inicialmente, vamos mostrar que $|C| \subseteq|B|$. Com esta finalidade, demonstramos an tes o seguinte resultado.

$\underline{\text { PROPOSICÃO } 3}-$ Seja $q$ em $Q_{C}$ e $\left\{q_{-}\right\}=I_{C}$. Para cada passeio $\mathrm{c}: \mathrm{q}_{-} \longrightarrow \mathrm{q}$ em $\mathrm{C}$ e para cada $\mathrm{s}$ em $\Sigma_{\mathrm{C}}^{*}$, se $(1, \mathrm{~s})|\mathrm{c}|=\left(\mathrm{y}_{1}, \mathrm{y}_{2}\right)$, para $y_{1}$ e $y_{2}$ em $\mathrm{T}_{C}^{*}$, então existe um passeio $\mathrm{d}: t_{-} \longrightarrow \mathrm{q}$ em $B$, on de $\left\{t_{-}\right\}=I_{B}$, ta1 que $(1, s)|d|=\left(y_{1} \xi x, y_{2}\right)$, onde $x \in x^{*}$.

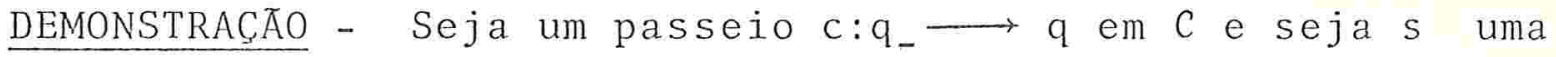
palavra em $\Sigma_{C}^{*}$ tal que

$$
(1, s)|c|=\left(y_{1}, y_{2}\right) \text {, para } y_{1} \text { e } y_{2} \text { em } T_{C}^{*} .
$$

Queremos mostrar que existe um passeio d:t_ $\rightarrow$ q em $B \quad$ tal que $(1, s)|d|=\left(y_{1} \xi x, y_{2}\right)$, onde $x \in x^{*}$.

Procedemos por indução no comprimento do passeio c.

$$
\begin{aligned}
& \text { Se } c \text { é o passeio trivial } c=1_{q_{-}} \text {, então } \\
(1, s)|c|= & (1, s), \text { ou seja, } y_{1}=1 \text { e } y_{2}=s . \\
& \text { Logo, } d=\left(t_{-}, E_{\xi}^{-1}, N, q_{-}\right) \text {è um passeio em } B \text { de } t_{-} \text {a }
\end{aligned}
$$

q_ tal que

$$
(1, s)|d|=(\xi, s), \text { com } x=1 \text {. }
$$


Suponhamos agora que $\mathrm{c}: \mathrm{q}_{-} \longrightarrow \mathrm{q}$ è um passeio não degenerado. Neste caso, existem arestas $\alpha_{1}, \alpha_{2}, \ldots, \alpha_{m}$ em $A_{c}$, para algum $m \geq 1$, tais que $c=\alpha_{1} \alpha_{2} \ldots \alpha_{m}$ e existem $u_{1}$ e $u_{2}$ em $\mathrm{T}_{\mathrm{C}}^{*}$ tais que

$$
(1, s)\left|c_{1}\right|=\left(u_{1}, u_{2}\right),
$$

onde $c_{1}=\alpha_{1} \ldots \alpha_{m-1}$ tem término em algum $p \in Q_{C}$ e de (8) segue que

$$
\left(u_{1}, u_{2}\right)\left|\alpha_{m}\right|=\left(y_{1}, y_{2}\right)
$$

Então pela hipótese da indução aplicada ao passeio $c_{1}$, existe em $B$ um passeio $d_{1}: t_{-} \longrightarrow p$ tal que

$$
(1, s)\left|d_{1}\right|=\left(u_{1} \xi x, u_{2}\right)
$$

onde $x \in x^{*}$.

Analisamos a seguir cada uma das duas possibilidades para $\alpha_{m}$ em $A_{C}$.

- se $\alpha_{m}$ é a i-êsima aresta de $c, \alpha_{m}=\left(p, N, i_{2}, q\right)$, para algum $i_{2}$ em $D_{C}$, então existe em $B$ o passeio $d: t_{-} \longrightarrow q$,

$$
\mathrm{d}=\mathrm{d}_{1} \beta_{1} \beta_{2},
$$

$\operatorname{com} \beta_{1}=\left(p, N, i_{2},(q, i)\right)$ e $\beta_{2}=\left((q, i), E_{x_{i}}^{-1}, N, q\right)$.

Assim, utilizando (10) segue que

$$
\begin{aligned}
(1, s)|d| & =(1, s)\left|d_{1}\right|\left|\beta_{1}\right|\left|\beta_{2}\right| \\
& =\left(u_{1} \xi x, u_{2}\right)\left|\beta_{1}\right|\left|\beta_{2}\right| .
\end{aligned}
$$


Mas, de (9) temos que $u_{1}=y_{1}$ e $u_{2} i_{2}=y_{2}$.

$$
\begin{aligned}
\operatorname{Logo},(1, s)|d| & =\left(y_{1} \xi x, y_{2}\right)\left|\beta_{2}\right| \\
& =\left(y_{1} \xi x x_{i}, y_{2}\right),
\end{aligned}
$$

onde $x x_{i} \in X^{*}$.

- se $\alpha_{m}$ é a i-ésima aresta de $C, \alpha_{m}=\left(p, i_{1}, N, q\right)$, para algum $i_{1}$ em $E_{C}$ e $x=z_{1} z_{2} \cdots z_{m-1}$, com $z_{j}$ em $X(1 \leq j \leq m-1)$, então sendo

$$
\begin{aligned}
& \mathrm{d}_{2}=\left(\mathrm{p}, \mathrm{N}, \mathrm{D}_{\$}^{-1},\left(\mathrm{p}, \sigma_{1}\right)\right) \\
& \mathrm{d}_{3}=\left(\left(\mathrm{p}, \sigma_{1}\right), \mathrm{E}_{\mathrm{z}_{\mathrm{m}-1}}, \mathrm{~N},\left(\mathrm{p}, \mathrm{z}_{\mathrm{m}-1}\right)\right)\left(\left(\mathrm{p}, \mathrm{z}_{\mathrm{m}-1}\right), \mathrm{N}, \mathrm{D}_{\mathrm{z}_{\mathrm{m}-1}}^{-1},\left(\mathrm{p}, \sigma_{1}\right)\right) \ldots \\
& \ldots\left(\left(p, \sigma_{1}\right), E_{z_{1}}, N,\left(p, z_{1}\right)\right)\left(\left(p, z_{1}\right), N, D_{z_{1}}^{-1},\left(p, \sigma_{1}\right)\right) \\
& \mathrm{d}_{4}=\left(\left(p, \sigma_{1}\right), E_{\xi}, N,\left(p, \sigma_{2}\right)\right) \\
& \mathrm{d}_{5}=\left(\left(p, \sigma_{2}\right), \mathrm{i}_{1}, \mathrm{~N},\left(\mathrm{q}, \sigma_{3} \mathrm{i}\right)\right) \\
& d_{6}=\left(\left(q, \sigma_{3}, i\right), E_{\xi}^{-1}, N,\left(q, \sigma_{4}, i\right)\right), \\
& d_{7}=\left(\left(q, \sigma_{4}, i\right), N, D_{z_{1}},\left(q, z_{1}, i\right)\right)\left(\left(q, z_{1}, i\right), E_{z_{1}}^{-1}, N,\left(q, \sigma_{4}, i\right)\right) \ldots \\
& \ldots\left(\left(q, \sigma_{4}, i\right), N, D_{z_{m-1}},\left(q, z_{m-1}, i\right)\right)\left(\left(q, z_{m-1}, i\right), E_{z_{m-1}}^{-1}, N,\left(q, \sigma_{4}, i\right)\right) \text {, } \\
& d_{8}=\left(\left(q, \sigma_{4}, i\right), N, D_{\$},\left(q, \sigma_{5}, i\right)\right) \\
& \text { e } d_{9}=\left(\left(q, \sigma_{5}, i\right), E_{x_{i}}^{-1}, N, q\right) \text {, }
\end{aligned}
$$

resulta que

$$
\mathrm{d}=\mathrm{d}_{1} \mathrm{~d}_{2} \mathrm{~d}_{3} \mathrm{~d}_{4} \mathrm{~d}_{5} \mathrm{~d}_{6} \mathrm{~d}_{7} \mathrm{~d}_{8} \mathrm{~d}_{9}
$$


è um passcio em $B$ de $t_{\text {_ }}$ a q e usando (10) e as definições dos passeios $\mathrm{d}_{2}, \mathrm{~d}_{3}$ e $\mathrm{d}_{4}$, obtemos que

$$
\begin{aligned}
(1, \mathrm{~s})|\mathrm{d}| & =(1, \mathrm{~s})\left|\mathrm{d}_{1}\right| \ldots\left|\mathrm{d}_{9}\right| \\
& =\left(u_{1} \xi x, \mathrm{u}_{2}\right)\left|\mathrm{d}_{2}\right| \ldots\left|\mathrm{d}_{9}\right| \\
& =\left(\mathrm{u}_{1} \xi x, \$ \mathrm{u}_{2}\right)\left|\mathrm{d}_{3}\right| \ldots\left|\mathrm{d}_{9}\right| \\
& =\left(u_{1} \xi, \times \$ \mathrm{u}_{2}\right)\left|\mathrm{d}_{4}\right| \ldots\left|\mathrm{d}_{9}\right| \\
& =\left(u_{1}, \times \mathrm{u}_{2}\right)\left|\mathrm{d}_{5}\right| \ldots\left|\mathrm{d}_{9}\right| .
\end{aligned}
$$

Mas, de (9) segue que $u_{1} i_{1}=y_{1}$ e $u_{2}=y_{2}$.

Então,

$$
\begin{aligned}
(\mathrm{s}, 1)|\mathrm{d}| & =\left(\mathrm{y}_{1}, \mathrm{x} \$ \mathrm{y}_{2}\right)\left|\mathrm{d}_{6}\right| \ldots\left|\mathrm{d}_{9}\right| \\
& =\left(\mathrm{y}_{1} \xi, x \$ y_{2}\right)\left|\mathrm{d}_{7}\right|\left|\mathrm{d}_{8}\right|\left|\mathrm{d}_{9}\right| \\
& =\left(y_{1} \xi x, \$ y_{2}\right)\left|\mathrm{d}_{8}\right|\left|\mathrm{d}_{9}\right| \\
& =\left(y_{1} \xi x, y_{2}\right)\left|\mathrm{d}_{9}\right| \\
& =\left(y_{1} \xi \times x_{i}, y_{2}\right)
\end{aligned}
$$

onde $\mathrm{xx}_{i} \in X^{*}$. Assim, temos demonstrado a Proposição 3.

Retornando à demonstração do Teorema 1 , seja $s \in|C|$. Então, existe em $C$ um passeio $c: q_{-} \longrightarrow q_{+}$, onde $\left\{q_{-}\right\}=I_{C} \quad$ e $q_{+} \in F_{C} \quad$ tal que 


$$
(1, \mathrm{~s})|\mathrm{c}|=(y, 1) \text {, para algum } y \text { em } \mathrm{T}_{\mathrm{C}}^{*} \text {. }
$$

Logo, pela proposição que acabamos de demonstrar scgue que existe em $B$ um passeio $d: t_{-} \longrightarrow q_{+}$, onde $\left\{t_{-}\right\}=I_{B}$ tal que

$$
(1, s)|d|=(y \xi x, 1), \text { com } x \in X^{*} \text {. }
$$

Mas, por construção de $B$, o estado $q_{+}$pertence a $F_{B}$ e $y \xi x \in \mathrm{T}_{B}^{*}$. Portanto, $s \in|B| \mathrm{e}|C| \subseteq|B|$. Analogamente, demonstra-se que $|B| \subseteq|C|$. Assim, $|B|=|C|=|A|$ e completamos a demonstração do Teorema 1 .

EXEMPLO 6 - Consideremos a seguinte māquina de Turing deterministica

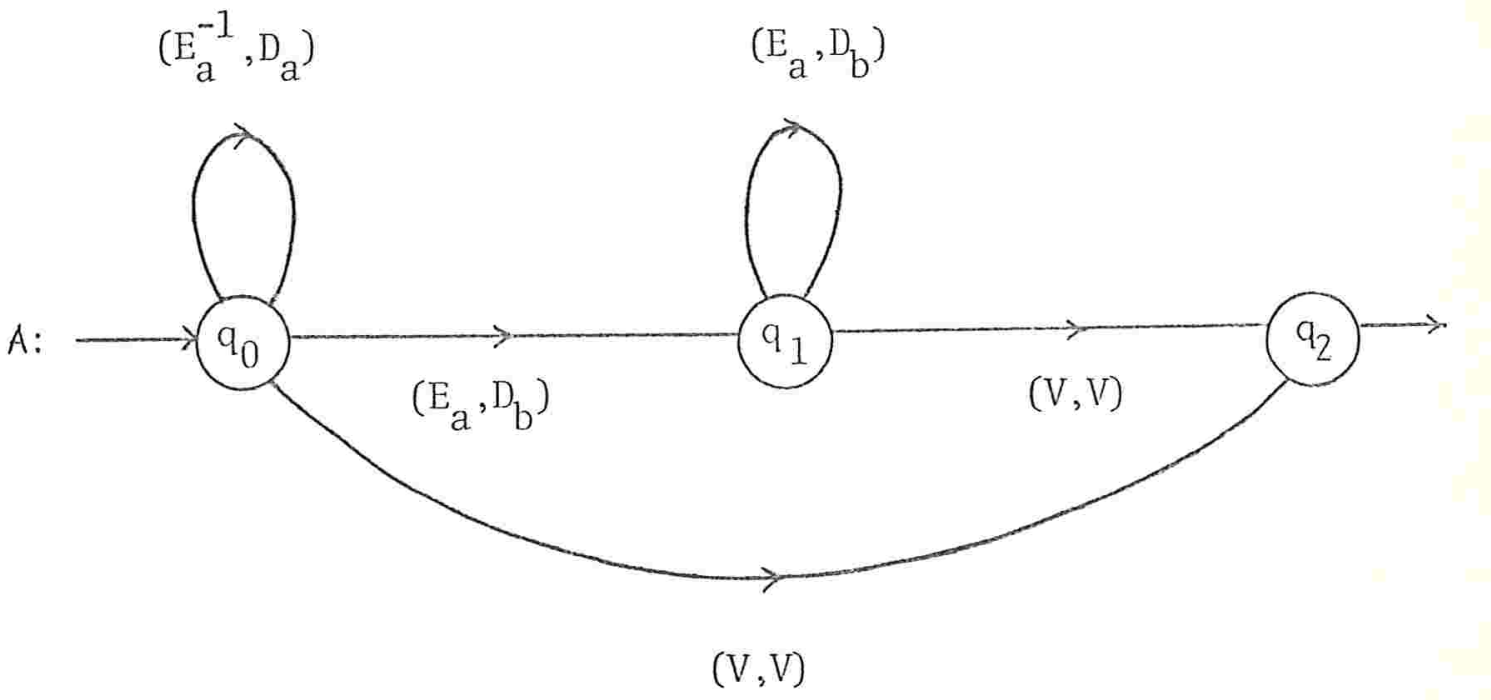

Pelo Lema 1 obtemos uma mâquina de Turing determinística e se 
mi-normalizada $B$ tal que $|A|=|B|$.

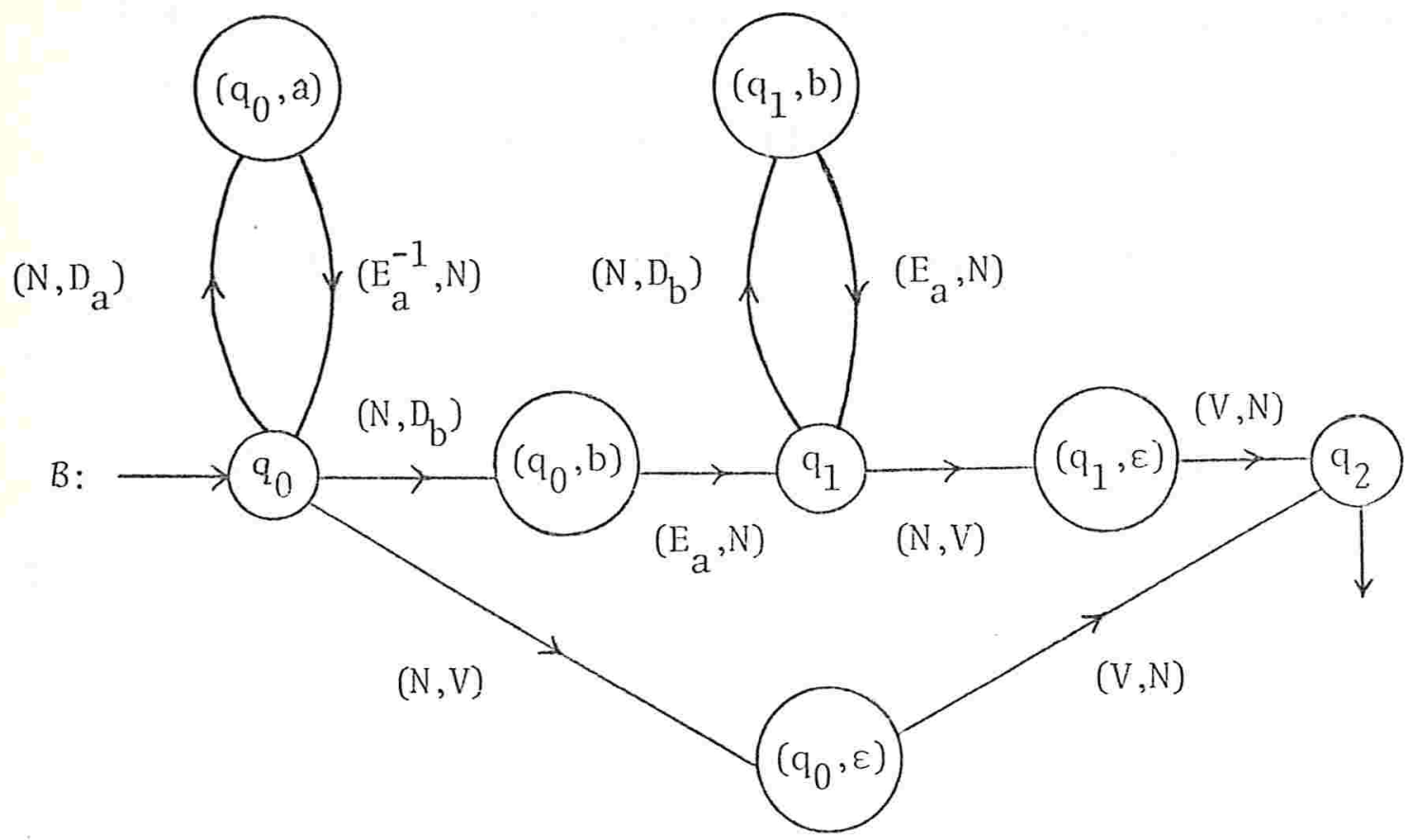

Utilizando o Lema 2 e redenominando os estados $\left(q_{0}, a\right),\left(q_{0}, b\right)$, $\left(q_{0}, \varepsilon\right),\left(q_{1}, b\right)$ e $\left(q_{1}, \varepsilon\right)$ por $q_{3}, q_{4}, q_{5}, q_{6}$ e $q_{7}$, respectivamen te, obtemos uma máquina de Turing determinística e normalizada $C$ tal que $|C|=|B|$.

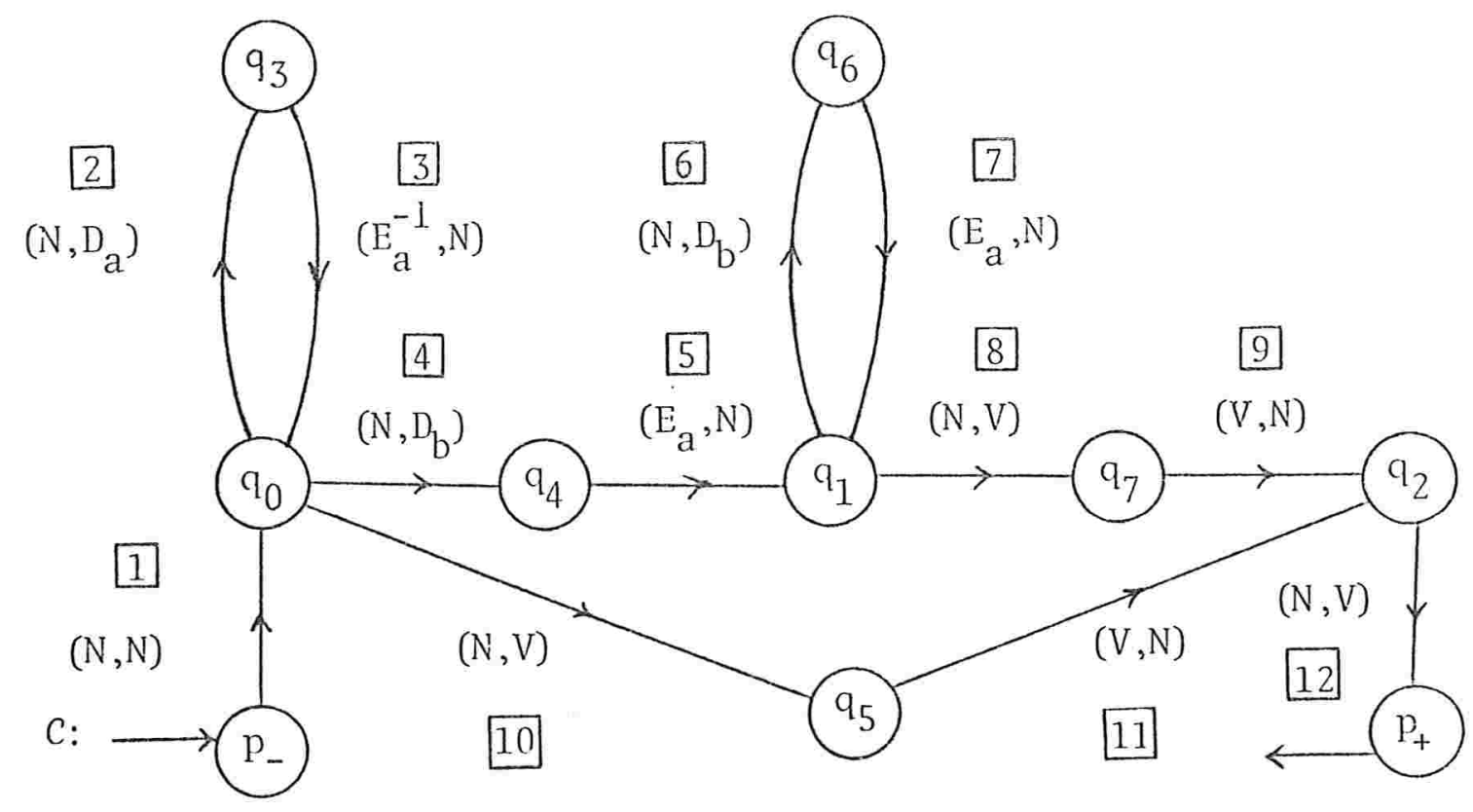


Os nümeros envolvidos por um $\square$ representam uma cnumeração das arestas de $C$.

Aplicando-se o Teorema 1 à máquina de Turing $\mathrm{C}$, ob temos uma máquina de Turing reversível e normalizada $D$ tal que $|C|=|D|$

Na representação gráfica da mãquina de Turing $D$ utiljzamos um $\square$ ao invës de exibir todos os 12 estados, sendo que cada estado corresponde a um dos $x_{i}, 1 \leq i \leq 12$. 


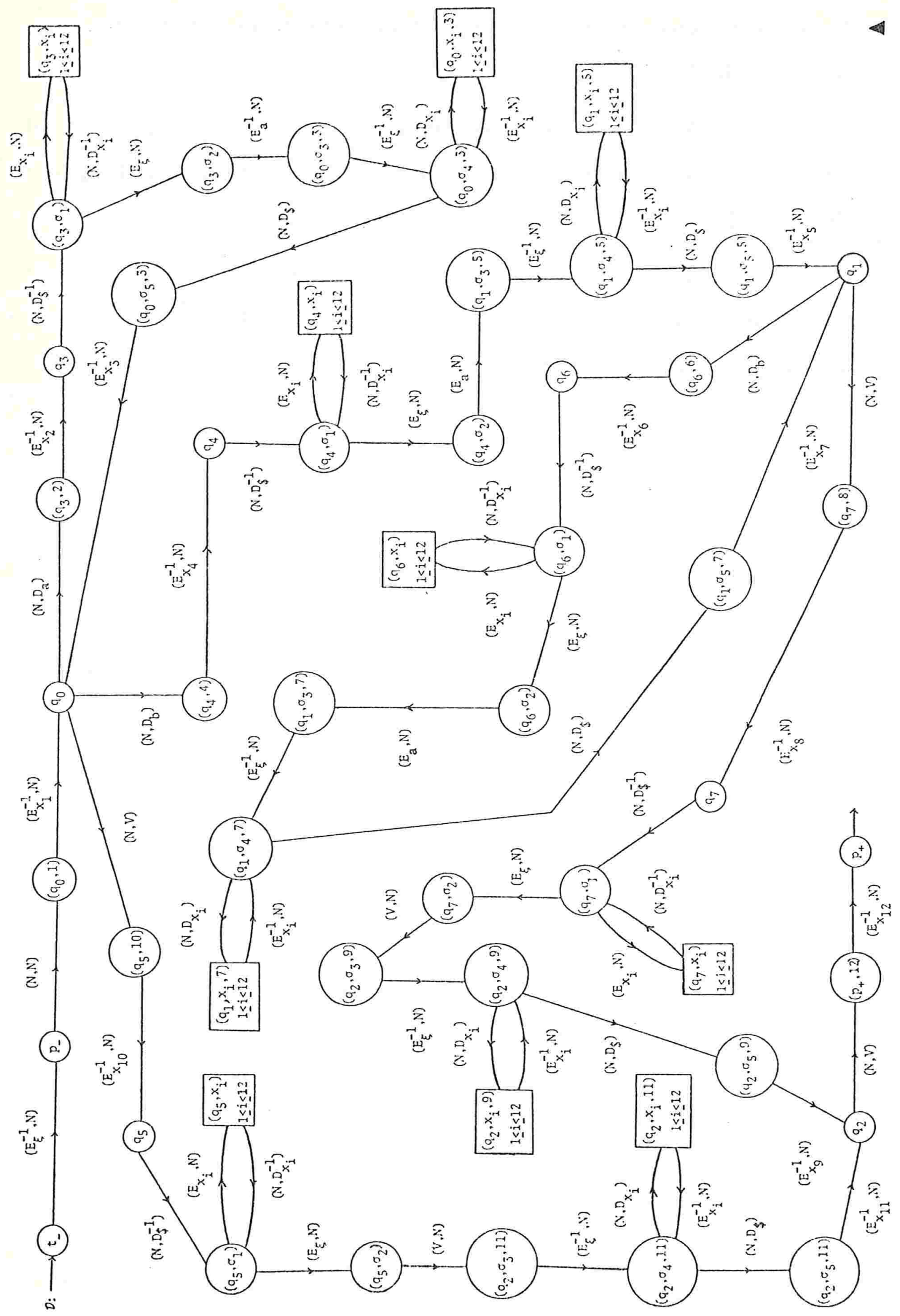


5. ACOPLAMENTO DE UMA MÃQUTNA DE TURING RLEVIRSTVIIL I: NORMALIZADA COM A SUA REVERSA.

A mäquina de Turing que construimos a seguir será essencial na demonstração dos dois resultados principais desta dissertação.

Seja A uma mãquina de Turing reversível e normalizada; seja $A^{\prime}$ a reversa de $A$. Está claro que $A^{\prime}$ também é reversível e normalizada. Denotemos o estado final de $A$ por $q_{+}$. Então, o acoplamento de A com $A^{\prime}$ è a mäquina de Turing $B$ com

$$
\begin{aligned}
\mathrm{T}_{B} & =\mathrm{T}_{A}, \\
\Sigma_{B} & =\Sigma_{A}, \\
Q_{B} & =Q_{A} \cup Q_{A^{\prime}}, \\
I_{B} & =I_{A}, \\
F_{B} & =F_{A}^{\prime} \\
\text { e } & =A_{A} U A_{A}, U\left\{\left(q_{+}, N, V, q_{+}^{\prime}\right)\right\} .
\end{aligned}
$$

E imediato que B também é reversível e normalizada. Esquemati camente, a mãquina de Turing $B$ pode ser representada como segue. 


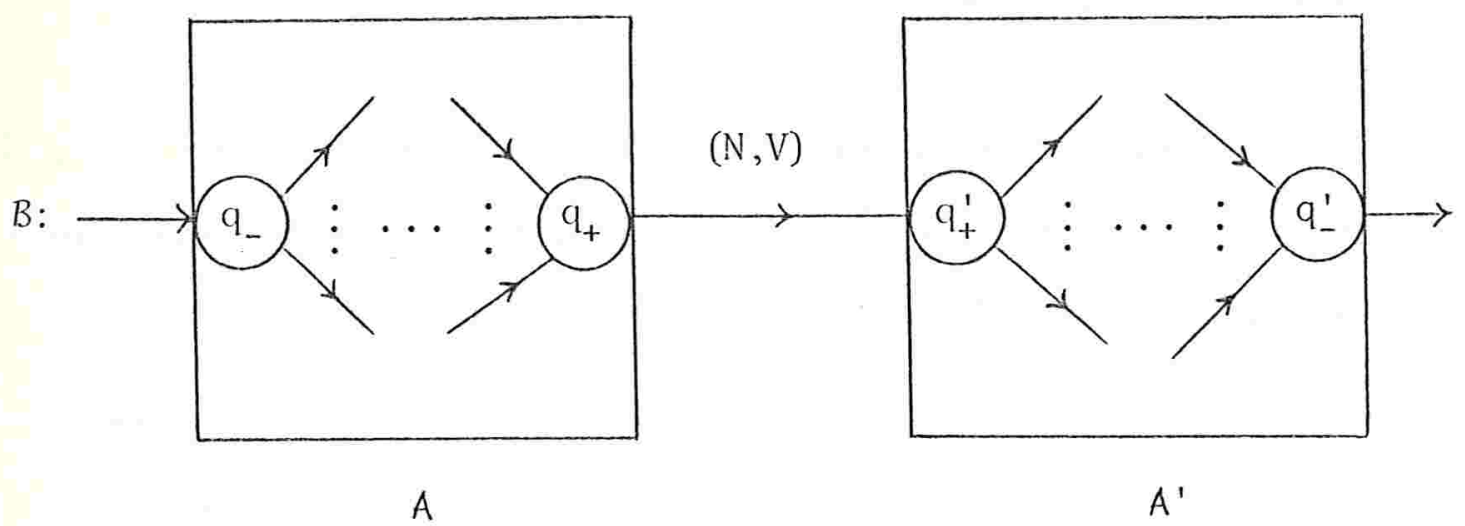

Atravês da próxima proposição descrevemos a relação existente entre as máquinas $A$ e $B$, ou seja, se $\left(s_{0}, t_{0}\right)$, com $s_{0}, t_{0}$ em $\mathrm{T}_{A}^{*}$, é uma configuração inicial para $A$ então a ca da passeio $d: q_{-} \longrightarrow q_{+}$em A estā associado o passeio $c: q_{-} \rightarrow q^{\prime}$ em $B$ tal que $c=d\left(q_{+}, N, V, q_{+}^{\prime}\right) d:$ e $\left(s_{0}, t_{0}\right)|c|=\left(s_{0}, t_{0}\right)$; reciprocamente, para cada passeio $c: q_{-} \longrightarrow q_{-}^{\prime}$ em $B$ existe um passeio $d: q_{-} \longrightarrow q_{+}$em $A$ tal que $c=d\left(q_{+}, N, V, q_{+}^{\prime}\right) d^{\prime}$.

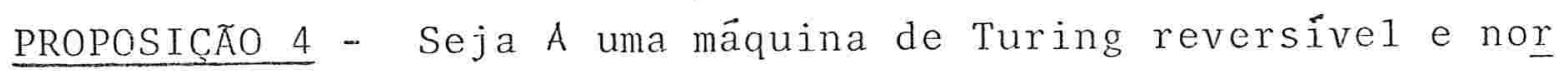
malizada, com $I_{A}=\left\{q_{-}\right\}$. Seja $B$ o acoplamento de $A$ com a sua reversa $A^{\prime}$. Então, para cada palavra $s$ em $\Sigma_{A}^{*}, s \in|A|$ sse $B$ tem um passeio $c: q_{-} \longrightarrow q_{-}^{\prime}$ tal que $\left(\varepsilon q_{-} s \varepsilon\right)|| c||=\varepsilon q_{-}^{\prime} s \varepsilon$.

DEMONSTRAÇÃO - Seja A uma máquina de Turing reversível e nor malizada com $I_{A}=\left\{q_{-}\right\}$e $F_{A}=\left\{q_{+}\right\}$; seja $A^{\prime}$ a reversa de $A$. Consideremos a máquina de Turing $B$ como sendo $O$ acoplamento de $A$ com $A^{\prime}$ e seja s uma palavra em $\Sigma_{A}^{*}$.

Inicialmente, suponhamos que $s \in|A|$. Então, A tem 
un passeio $\mathrm{d}_{\mathrm{a}} \mathrm{q}_{-} \longrightarrow \mathrm{q}_{+}$tal que

$$
\left(\varepsilon q_{-} s \varepsilon\right)|| d||=\varepsilon \operatorname{tq}{ }_{+} \varepsilon \text {, para algum } \mathrm{t} \text { em } \mathrm{T}_{A}^{*} \text {. }
$$

E pela Proposição 2, o passeio $d^{\prime}: q_{+}^{\prime} \longrightarrow q_{-}^{\prime}$ é o reverso de d em $A^{\prime} \quad \mathrm{e}$

$$
\left(\varepsilon \operatorname{tq} q_{+}^{\prime} \varepsilon\right)|| d^{\prime}||=\varepsilon q_{-}^{\prime} s \varepsilon
$$

Mas, os passeios d e d' também são passeios em B.A1ém disso, a aresta $\alpha=\left(q_{+}, N, V, q_{+}^{\prime}\right) \in A_{B}$. Logo, $c=$ dad' $\vec{e}$ um passeio em $B$ com origem em $q_{-}$e término em q! tal que

$$
\begin{aligned}
\left(\varepsilon q_{-} s \varepsilon\right)|| c|| & =\left(\varepsilon q_{-} s \varepsilon\right)|| d|||| \alpha|||| d^{\prime}|| \\
& =\left(\varepsilon t_{+} \varepsilon\right)|| \alpha|||| d^{\prime}|| \\
& =\left(\varepsilon \mathrm{tq}_{+}^{\prime} \varepsilon\right)|| d^{\prime}|| \\
& =\varepsilon q_{-}^{\prime} s \varepsilon .
\end{aligned}
$$

Reciprocamente, seja s em $\Sigma_{A}^{*}$ e suponhamos que $B$ tem um passeio $c: q_{-} \longrightarrow q_{-}^{\prime}$ tal que

$$
\left(\varepsilon q_{-} s \varepsilon\right)|| c||=\varepsilon q_{-}^{\prime} s \varepsilon \text {. }
$$

Mas, por construção de $B$ resulta que existem passejos $d: q_{-} \rightarrow q_{+}$ em $A$ e $d^{\prime}: q_{+}^{\prime} \longrightarrow q_{-}^{\prime}$ em $A^{\prime}$ tais que $c=d \alpha d^{\prime}$, onde $\alpha=\left(q_{+}, N, V, q_{+}^{\prime}\right) \in A_{B}$. Logo, de (11) e da Proposição 2 segue que existem palavras x e y em $\mathrm{T}_{A}^{*}$ tais que

$$
\left(\varepsilon q_{-.} s \varepsilon\right)|| d||=\varepsilon x q_{+} y \varepsilon
$$




$$
\begin{aligned}
& \left(\varepsilon \times q_{+} y \varepsilon\right)|| \alpha||=\varepsilon x q_{+}^{\prime} \varepsilon \text { e } y=1 \\
& \left(\varepsilon \times q_{+}^{\prime} \varepsilon\right)|| d^{\prime}||=\varepsilon q_{-}^{\prime} s \varepsilon .
\end{aligned}
$$

Assim, A tem um passeio $\mathrm{d}_{\mathrm{a}} \mathrm{q}_{-} \longrightarrow \mathrm{q}_{+}$tal que

$$
\left(\varepsilon q_{-} s \varepsilon\right)|| d||=\varepsilon \times q_{+} \varepsilon \text {, para algum } x \text { em } T_{A}^{*} \text {, ou seja, }
$$
$s \in|A|$.

Concluímos então a demonstração da Proposição 4. 


\section{CAPITULO 3}

\section{CASOS RESTRITOS DO PROBLEMA DE CORRESPONDENCIA DE POST}

Inicialmente, construimos dois morfismos associadosa cada mâquina de Turing semi-normalizada e provamos que se a måquina ê reversível então os morfismos associados são côdj-gos de atraso um. En seguida, definimos a codificaçäo de uma computação associada a um passeio não degenerado. Esses con ceitos são de importância vital para o restante deste trabalko. Assim, demonstramos o resultado principal deste capí tulo que é a indecidibilidade do Problema de Correspondência de Post quando restrito a côdigos de atraso um. Alëm disso," provamos que esses cödigos pertencem a PS $\cap S P$.

Podemos observar que as demonstrações de algumas pro posições poderiam ser simplificadas, mas o nosso objetivo ë 
apresentâ-las de modo que possam ser utilizadas também na pro va do teorema principal do capítulo seguinte.

Finalizando, expomos a condição (decidível, indecidí vel ou em aberto) de outros casos restritos do problema de Correspondência de Post.

\section{INTRODUCÃO}

Inicialmente vamos enunciar o

Problema de Correspondência de Post:

"Dados morfismos $h_{1}$ e $h_{2}$ de monöides livres, $h_{1}: h_{2}: \Gamma^{*} \longrightarrow \Delta^{*}$, determinar se existe ou não uma palavra não vazia $w$ em $\Gamma^{*}$ tal que $w h_{1}=w_{2} h^{\prime}$.

Alternativamente, o Problema de Correspondência de post pode ser descrito como segue.

Seja $\Gamma$ um alfabeto com n simbolos, $\Gamma=\left\{\gamma_{1}, \gamma_{2}, \ldots, \gamma_{n}\right\}$. Sejam duas $n$-uplas de palavras não vazias $\left(x_{1}, x_{2}, \ldots, x_{n}\right)$ e $\left(y_{1}, y_{2}, \ldots, y_{n}\right)$ sobre algum alfabeto $\Delta$, de modo que para cada i, $1 \leq i \leq n$, os morfismos $h_{1}$ e $h_{2}$ são tais que $x_{i}=\gamma_{i} h_{1}$ e $y_{i}=\gamma_{i} h_{2}$ Então, para cada $w \in m \Gamma^{+}, w=\gamma_{i_{1}} \gamma_{i_{2}} \cdots \gamma_{i_{k}} \quad, \operatorname{com} k \geq 1 \quad$ e $1 \leq i_{j} \leq n \quad(1 \leq j \leq k)$, temos que

$$
w h_{1}=x_{i_{1}} x_{i_{2}} \cdots x_{i_{k}} \quad \text { e } \quad w h_{2}=y_{i_{1}} y_{i_{2}} \cdots y_{i_{k}} \text {. }
$$


Assim, o Problema de Correspondência de Post consiste em determinar se existe ou não uma sequência de inteiros $i_{1}, i_{2}, \ldots, i_{\ell}(\ell \geq 1)$, com $1 \leq i_{j} \leq n$, para cada $j(1 \leq j \leq \ell)$, tal que

$$
{ }_{i_{1}} x_{i_{2}} \cdots x_{i_{\ell}}=y_{i_{1}} y_{i_{2}} \cdots y_{i_{\ell}} .
$$

Podemos observar que o conjunto de soluções da ins tância do Problema de Correspondência de Post com as n-uplas $\left(x_{1}, x_{2}, \ldots, x_{n}\right)$ e $\left(y_{1}, y_{2}, \ldots, y_{n}\right)$ representa o conjunto $\mathrm{Eq}\left(\mathrm{h}_{1}, \mathrm{~h}_{2}\right) \backslash\{1\}$.

Este problema, quando $h_{1}$ e $h_{2}$ são morfismos quaisquer, foi mostrado ser indecidivel por Post (1946).

Chomsky e Schutzenberger (1963) mostraram que este problema permanece indecidivel quando un dos morfismos $\vec{e}$ inje tor e Schutzenberger conjeturou que, mesmo considerando ambos os morfismos injetores, este problema permaneceria jndecidi vel.

Assim, Lecerf (1963b) provou que a conjetura de Schutzenberger é välida ou seja:

TEOREMA 1 - Lecerf

o Problema de Correspondēncia de Post ẻ indecidí vel mesmo quando restrito a mosfismos injetores.

Com a finalidade de demonsträ-1o, Lecerf introduziu o conceito de mäquinas de Turing reversiveis. Em seguida, pa 
ra uma dada 1 inguagem recursivamente enumerảvel $L \subseteq \Sigma^{*}$ associou uma måquina de Turing reversível A tal que $|A|=L$ e cons truiu uma outra mâquina de Turing reversível $B$ que è a "conca tenação" de A com a sua reversa A'. Então, associou a B dois morfismos injetores $h_{1}$ e $h_{2}$ e reduziu o Problema da Parada pa ra o Problema de Correspondência de Post com esses morfismos, demonstrando que para cada palavra $\mathrm{s}$ em $\Sigma^{*}$, a máquina de $\mathrm{Tu}$ ring A pära com entrada s sse existe uma palavra $x$ na imagem de $h_{1}$ tal que $x=x h$, onde $h=h_{1}^{-1} h_{2} ;$ ou seja, Lecerf determinou uma palavra $x$ que fosse igual a sua imagem por $h$, ao invês de encontrax uma palavra y cujas imagens por $h_{1}$ e $h_{2}$ fossem iguais. - Teorema segue então do fato de que o Problema da parada é indecidjuvel.

Nós estendemos o resultado de Lecerf, mostrando que - Problema de Correspondência de Post permanece indecidivel mesmo quando restringimos ambos os morfismos $h_{1}$ e $h_{2}$ para serem côdigos de atraso um.

Este è o resultado principal deste capĩtulo; vamos apenas enunciā-1o aqui e demonstrá-1o-emos mais tarde.

TEOREMA 2 - O Problema de Correspondência de Post é indecidivel mesmo quando restrito a côdigos de atraso um. 
2. CONSTRUCKO DE MORFISMOS ASSOCIADOS A UMA MX̃OUINA DE TURING SEMI-NORMALIZADA

O objetivo desta seção é associar dois morfismos a uma måquina de Turing semi-normalizada e investigar quais são as propriedades apresentadas por esses morfismos caso a måqui na de Turing considerada seja reversivel.

Seja A uma máquina de Turing semi-normalizada e sejam os alfabetos

$$
\Gamma=\mathrm{T}_{A} \cup\{\varepsilon, \bar{H}\} \cup \mathrm{A}_{A} \cup \overline{\mathrm{T}}_{A} \cup\{\bar{\varepsilon}, \overline{\bar{A}}\} \cup \overline{\mathrm{Q}}_{\mathrm{A}}
$$

$\mathrm{e} \quad \Delta=\mathrm{T}_{\mathrm{A}} \cup\{\varepsilon, A\} \cup \mathrm{Q}_{A} \cup \overline{\mathrm{T}}_{\mathrm{A}} \cup\{\bar{\varepsilon}, \overline{\mathrm{B}}\} \cup \ddot{Q}_{A}$,

onde $\varepsilon e$ são simbolos distintos que não pertencem a $T_{A} \cup Q_{A}$. Consideremos os morfismos $f$ e $g: \Gamma^{*} \rightarrow \Delta^{*}$, definidos pela Tabela 1 , onde o denota um si̊mbolo em $\mathrm{T}_{A}, q$ e $r$ são elementos de $Q_{A}$ e $(q, i, j, r)$, com $i \in E_{A}$ e $j \in D_{A}$, representa uma aresta em $A_{A}$.

Na verdade, a cada māquina de Turing semi-normalizada A basta considerar o morfismo $g$, pois o morfismo $f$ corresponde (a menos de "barras" e "linhas") ao morfismo g' associa do a måquina de Turing $A^{\prime}$, que è a reversa de $A$. E o que vamos verificar a seguir.

Seja. $A^{\prime}$ a máquina de Turing reversa de $A$ e sejam os alfabetos 


\begin{tabular}{|c|c|c|}
\hline$\gamma$ & $\gamma f$ & $\gamma g$ \\
\hline o & $\bar{\sigma}$ & $\sigma$ \\
\hline $\bar{\sigma}$ & $\sigma$ & $\bar{\sigma}$ \\
\hline$\varepsilon$ & $\bar{\varepsilon}$ & $\varepsilon$ \\
\hline $\bar{\varepsilon}$ & $\varepsilon$ & $\bar{\varepsilon}$ \\
\hline 鈢 & $\ddot{\theta}$ & \# \\
\hline 至 & $\Leftrightarrow$ & 蓓 \\
\hline$\left(q, E_{\sigma}, N, r\right)$ & $\vec{x}$ & $\sigma q$ \\
\hline$\left(q, N, D_{\sigma}, r\right)$ & $\bar{r}$ & $q c$ \\
\hline$(q, v, N, r)$ & $\bar{\varepsilon} \bar{r}$ & $\varepsilon q$ \\
\hline$(q, N, V, r)$ & $\bar{r} \bar{\varepsilon}$ & $q \varepsilon$ \\
\hline$\left(\mathrm{q}, \mathrm{E}_{\sigma}^{-1}, N, r\right)$ & $\overline{\sigma r}$ & $q$ \\
\hline$\left(q, N, D_{\sigma}^{-1}, r\right)$ & $\bar{r} \bar{\sigma}$ & $q$ \\
\hline$(q, N, N, r)$ & $\bar{r}$ & 9 \\
\hline $\bar{q}$ & $q$ & $\bar{q}$ \\
\hline
\end{tabular}

I'abela I - Definição dos morfismos associados a uma mäquina de Turing semi-normalizada. 


$$
r^{\prime}=T_{A} \cup\{\varepsilon, A\} \cup A_{A^{\prime}} \cup \bar{T}_{A} \cup\{\bar{\varepsilon}, \bar{H}\} \cup \bar{Q}_{A^{\prime}}
$$

e

$$
\Delta^{\prime}=T_{A} \cup\{\varepsilon, \text { a }\} \cup Q_{A^{\prime}} \cup \bar{T}_{A} \cup\{\bar{\varepsilon}, \bar{A}\} \cup \bar{Q}_{A^{\prime}} \text {. }
$$

Consideremos o morfismo $g^{\prime}:\left(\Gamma^{\prime}\right)^{*} \longrightarrow\left(\Delta^{\prime}\right)^{*}$, definido pela Tabela 2 , onde $\sigma$ denota um símbolo em $\mathrm{T}_{A}, q^{\prime} \mathrm{e} \mathrm{r}^{\prime}$ são elementos de $Q_{A^{\prime}}$ e $\left(r^{\prime}, i, j, q^{\prime}\right)$ com $i \in E_{A}$ e $j \in D_{A}$, represen ta uma aresta em $A_{A^{\prime}}$.

\begin{tabular}{|c|c|}
\hline$\gamma \in \Gamma^{\prime}$ & $r_{g^{\prime}}$ \\
\hline$\sigma$ & $\sigma$ \\
$\bar{\sigma}$ & $\bar{\sigma}$ \\
$\varepsilon$ & $\varepsilon$ \\
$\bar{\varepsilon}$ & $\bar{\varepsilon}$ \\
$\theta$ & $q^{\prime}$ \\
$\bar{q}$ & $\bar{q}$ \\
$\left(r^{\prime}, E_{\sigma}^{-1}, N, q^{\prime}\right)$ & $r^{\prime}$ \\
$\left(r^{\prime}, N, D-q^{\prime}, q^{\prime}\right)$ & $r^{\prime}$ \\
$\left(r^{\prime}, V, N, q^{\prime}\right)$ & $\varepsilon r^{\prime}$ \\
$\left(r^{\prime}, N, V, q^{\prime}\right)$ & $r^{\prime} \varepsilon$ \\
$\left(r^{\prime}, E, N, q^{\prime}\right)$ & $\sigma r^{\prime}$ \\
$\left(r^{\prime}, N, D, q^{\prime}\right)$ & $r^{\prime} \sigma$ \\
$\left(r^{\prime}, N, N, q^{\prime}\right)$ & $r^{\prime}$ \\
$\bar{q}^{\prime}$ & $\bar{q}$ \\
\hline
\end{tabular}

Tabeza 2 - Definição do morfismo g' associado à reversa de uma máquina de Turing semi-normalizada.

Sejam ainda os morfismos:

$$
\phi: \Gamma^{*} \longrightarrow\left(\Gamma^{\prime}\right)^{*},
$$


definido pela Tabela 3 , onde o é um sïmbolo em $\mathrm{T}_{A} \cup \bar{T}_{A} \cup\left\{\varepsilon,{ }^{H}, \bar{\varepsilon}, \bar{\varphi}\right\}$, $q$ è um elemento de $Q_{A} e(q, i, j, r)$, com $i \in E_{A}$ e $j \in D_{A}$, representa uma aresta em $A_{A}$

e $\psi:\left(\Delta^{\prime}\right) * \rightarrow \Delta^{*}$,

dado pela Tabela 4, onde o representa um simbolo em $\mathrm{T}_{\mathrm{A}} \cup\{\varepsilon, \forall\}$ e q' è um elemento de $Q_{A}$.

\begin{tabular}{|l|l|}
\hline$\gamma \in r$ & $\gamma \phi$ \\
\hline$\sigma$ & $\sigma$ \\
\hline $\bar{q}$ & $\bar{q}^{\prime}$ \\
$(q, i, j, r)$ & $\left(r^{\prime}, i^{-1}, j^{-1}, q^{\prime}\right)$ \\
\hline
\end{tabular}

Tabela 3 - Definição do morfismo $\phi$.

\begin{tabular}{|c|c|}
\hline$\delta \in \Delta^{\prime}$ & $\delta \psi$ \\
\hline$\sigma$ & $\bar{\sigma}$ \\
$\bar{\sigma}$ & $\sigma$ \\
$\mathrm{q}^{\prime}$ & $\overline{\mathrm{q}}$ \\
$\overline{\mathrm{q}}^{\prime}$ & $\mathrm{q}$ \\
\hline
\end{tabular}

Tabeza 4 - Definição do morfismo $\psi$

Observemos que $\phi$ e $\psi$ são bijeçëes. 
Assim, temos imediatamente o pröximo resultado.

PROPOSIÇRO $1-\mathrm{f}=\phi \mathrm{g}^{\prime} \psi$.

E importante ressaltarmos a dualidade existente entre os morfismos $f$ e $g$, o que simplificarä algumas demonstraçöes referentes às propriedades de $f$ e $g$.

A seguir, temos duas observações acerca dos morfis mos $f$ e $g$.

OBSERVACÃO 1 - Pode-se verificar que se a mäquina de Turing A não for deterministica, então o morfismo g poderā não ser injetor. Por exemplo, se $A_{A}$ contiver as arestas $a_{1}=\left(q, E_{0}, N, r_{1}\right)$ e $\alpha_{2}=\left(q, E_{\sigma}, N, r_{2}\right)$, com $r_{1} \neq r_{2}$, então $\alpha_{1} g=\alpha_{2} g=\sigma q$ ou se as arestas $\beta_{1}=\left(q, E_{\sigma}, N, r\right)$ e $\beta_{2}=\left(q, E_{\sigma}^{-1}, N, r\right)$ pertencerem a $A_{A}$ então $\beta_{1} g=\left(\sigma \beta_{2}\right) g=\sigma q$.

OBSERVAC̄̃O 2 - Se a máquina de Turing A não for reversĩvel, então $A^{\prime}$ poderả não ser deterministica. Neste caso, o morfismo $g^{\prime}$ poderâ não ser injetor e, consequentemente, o morfismo f poderä não ser injetor.

$\mathrm{Na}$ Proposição 2 mostramos a recíproca, ou seja, se A ê uma mäquina de Turing reversivel (e, portanto, deterministi ca) e semi-normalizada, então os morfismos $f$ e g são injetores.

Uma outra observação é com relação ao formalismo que adotamos para definirmos uma måquina de Turing. Podemos veri- 
ficar que as imagens das arestas por $f$ e por $g$ não precisam in dicar se o movimento da cabeça da fita é para a esquerda ou pa ra a direita ou ainda se permanece inalterado, como seria necessärio caso estivêssemos utilizando o formalismo usual.

A proposição a seguir apresenta-nos as propriedades dos morfismos associados a uma mäquina de Turing reversível e semi-normalizada.

PROPOSIÇAO 2 - Seja A uma mäquina de Turing reversĩvel e semi-normalizada. Então, ambos os morfismos associados a A são cödigos de atraso um.

DEMONSTRACÃO - Seja A uma máquina de Turing reversível e seni -normalizada. Sejam os alfabetos

$$
\Gamma=T_{A} \cup\{\varepsilon, \vec{A}\} \cup A_{A} \cup \overline{\mathrm{T}}_{A} \cup\{\bar{\varepsilon}, \overline{\bar{A}}\} \cup \overline{\mathrm{Q}}_{A}
$$$$
\text { e } \quad \Delta=T_{A} \cup\{\varepsilon, \theta\} \cup Q_{A} \cup \bar{T}_{A} \cup\{\bar{\varepsilon}, \bar{H}\} \cup \bar{Q}_{A} \text {. }
$$

Considerenos os morfismos $f$ e $g: r^{*} \rightarrow \Delta^{*}$ associados a $A$ e de finidos como na Tabela 1.

A demonstração da Proposição 2 segue então das quatro proposições a seguir.

PROPOSICAO $2 \mathrm{a}$ - 0 morfismo g ê injetor sobre $\Gamma$.

PROPOSICÃO 2b - o morfismo g è um código de atraso um. 
PROPOSICKO 2c - 0 morfismo $f \vec{e}$ injetor sobre $\Gamma$.

PROPOSICÃO 2d - 0 morfismo $f$ é um côdigo de atraso um.

\section{Demonstração da Proposição 2a}

$$
\begin{aligned}
& \text { Queremos mostrar que } g \text { ê injetor sobre } \Gamma \text {. } \\
& \text { Sejam } x \text { e } y \text { em } r \text { tais que } x g=y g \text {. }
\end{aligned}
$$

- Se $x g \in \mathrm{T}_{A} \cup \overline{\mathrm{T}}_{A} U\{\varepsilon, \bar{\varepsilon}, A, \bar{A}\} \cup \overline{\mathrm{Q}}_{A}$ segue pela definição de $g$ que $x=y$.

- Se $x g=q, q \in Q_{A}$, entäo $x$ è uma das arestas, $\left(q, E_{\sigma}^{-1}, N, r\right)$ ou $\left(q, N, D_{\sigma}^{-1}, r\right)$ ou $(q, N, N, r)$, e $x$ pertence a $A_{A}$. Supomhamos que $y \neq x$. Nesse caso, $y=\left(q, E_{\tau}^{-1}, N, t\right)$ ou $y=\left(q, N, D_{\tau}^{-1}, t\right)$ ou $y=(q, N, N, t)$, e $y$ pertence a $A_{A}$. Mas, como $A \vec{e}$ deterministica, $x$ e y não $p \underline{O}$ dem ocorrer simultaneamente em $A_{A}$. Logo, $x=y$.

- Seja $x g=\sigma q$, com o em $\mathrm{T}_{A}$ e $\mathrm{q}$ em $\mathrm{Q}_{A}$. Então, $x=\left(q, E_{\sigma}, N, r\right) \in A_{A}$. Se $y \frac{1}{t} x$, segue que $y=\left(q, E_{\sigma}, N, t\right) \in A_{A^{\prime}}$ para algum tłtr. 0 que è um absurdo, pois A ê deterministica. Logo, $x=y$.

- A demonstração é anāloga ’a anterior quando $x g=\varepsilon q$ ou $x g=q \sigma$ ou $x=q \varepsilon$, para $q$ em $Q_{A} e$ o em $T_{A}$.

Assim, $g \vec{e}$ um morfismo injetor sobre $\Gamma$. 


\section{Demonstração da Proposição 2b}

Queremos mostrar que o mosfirmo g é um código de atraso um.

Como pela Proposição $2 a \mathrm{~g} e \overrightarrow{~ i n j e t o r ~ s o b r e ~} \Gamma$, ẻ suficiente demonstrarmos que g é de atraso um.

Vamos, inicialmente, provar que $g$ é de atraso um da direjta para a esquerda.

Sejam u e $v$ em $r g$ e $w$ em $\Delta^{*}$ tais que wvu $G(r g) *$ En tão, existe $k \geq 1$ e $z_{1}, z_{2}, \ldots, z_{k}$ en $r g$ tais que

$$
w v u=z_{k} z_{k-1} \cdots z_{1}
$$

Precisamos mostrar que wV tambëm pertence a ( $\mathrm{rg}$ ) . Com esta fi nalidade, analisamos cada uma das possibilidades para u en $\Gamma$.

- Seja u $\in \overline{\mathrm{T}}_{A} \cup\{\bar{\varepsilon}, \boldsymbol{A}, \overline{\bar{H}}\} \cup \overline{\mathrm{Q}}_{A}$.

Nesse caso, $\vec{e}$ imediato verificar que $z_{1}=u$, pois pela definição de $g$ não existe uma palavra $t$ em $\Delta^{+}$tal que $z_{1}=$ tuerg. Logo, de (1) segue que $w v=z_{k} \ldots z_{2} \in(\Gamma g) *$.

- Seja $u=\sigma \quad$ em $\mathrm{T}_{A}$.

Suponhamos que $z_{1} \neq u$. Então, pela definição de g temos que $z_{1}=q \sigma$, para algum $q$ em $Q_{A}$ e a aresta $\alpha=\left(q, N, D_{\sigma}, r\right) \in A_{A}$, com $\alpha g=z_{1}$. E de (1) segue que pode ocorrex um dos seguintes ca sos:

(i) $\quad \mathrm{v}=\mathrm{q}$.

Nesse caso, uma das arestas $B=\left(q, E_{\delta}^{-1}, N, t\right)$ ou 


$$
\beta=\left(q, N, D_{\delta}^{-1}, t\right) \text { ou } \beta=(q, N, N, t) \text { pertence a } \Lambda_{A} \text { e } \beta g=v \text {. }
$$

(ii) $v=\tau q$, para algum $\tau$ em $\mathrm{T}_{A}$.

Então, a aresta $B=\left(q, E_{\tau}, N, t\right) \in \Lambda_{A}$ e $B g=v$.

(iii) $v=\varepsilon q$.

Então, a aresta $\beta=(q, V, N, t) \in A_{A}$ e $B g=v$.

Mas, qualquer um desses casos contradiz a hipötese de que A ê deterministica. Logo, $z_{1}=u$ e de (1) segue que wV $\in(\Gamma g)$ *.

- Seja $u=\varepsilon$.

A demonstração é obtida de modo análogo a anterior.

- Seja $u=\sigma q$, $\sigma$ em $\mathrm{T}_{\mathrm{A}}$ e $\mathrm{q}$ em $\mathrm{Q}_{\mathrm{A}}$.

Então, a aresta $\alpha=\left(q, E_{\sigma}, N, r\right) \in A_{A} e \alpha g=u$. Se $z_{1} \neq u$, resulta pela definição de g que $z_{1}=q$ e uma das arestas $\beta=\left(q, E_{\tau}^{-1}, N, t\right)$ ou $\beta=\left(q, N, D_{\tau}^{-1}, t\right)$ ou $\beta=(q, N, N, t)$ pertence a $A_{A}, \operatorname{com} B g=z_{1}$. Porêm, como $A$ è determinística, é imposs î.vel que isto ocorra. Logo, $z_{1}=u$ e de (1) segue que wve(rg)*.

- Seja u=eq $\in \varepsilon Q_{A}$.

A demonstação é anảloga a anterior.

- Seja $u=q \in Q_{A}$.

Nesse caso, uma das arestas $\alpha=\left(q, E_{\sigma}^{-1}, N, r\right)$ ou $\alpha=\left(q, N, D_{\sigma}^{-1}, r\right)$ ou $\alpha=(q, N, N, r)$ pertence a $A_{A}$ e $\alpha g=u$. Suponhamos que $z_{1} \neq u$. Então, pela definição de $g$ segue que $z_{1}=\tau q$, para algum $\tau$ em $\mathrm{T}_{\mathrm{A}}$ ou $\mathrm{z}_{1}=\varepsilon \mathrm{q}$. Logo, resulta, respectivamente, que a ares ta $\beta=\left(q, E_{\tau}, N, t\right)$ ou $\beta=(q, V, N, \tau)$ pertence a $A_{A}$ e $\beta g=z_{I} \cdot 0$ 
que contradiz a hipótese de que $A \vec{e}$ deterministica. Portan to, $z_{1}=u$ e de (1) segue que wv $\in(r g)^{*}$.

- Seja $u=q \sigma, q$ em $Q_{A}$ e $\sigma$ em $T_{A}$.

Então, a aresta $\alpha=\left(q, N, D_{\sigma}, r\right) \in A_{A}$ e $\alpha g=u$. Supondo-se que $z_{1} \neq v$, segue pela definição de $g$ e de (1) que $z_{1}=\sigma$ e as pos sibilidades para $z_{2}$ são:

(i) $\quad z_{2}=q$.

Então, uma das arestas $\beta=\left(q, E_{\delta}^{-1}, N, t\right)$ ou $\beta=\left(q, N, D_{\delta}^{-1}, t\right)$ ou $\beta=(q, N, N, t)$ pertence a $A_{A}$ e $B g=z_{2}$.

(ii) $z_{2}=\tau q$, para algum $\tau$ em $\mathrm{T}_{\mathrm{A}}$. Nesse caso, a aresta $B=\left(q, E_{\tau}, N, t\right) \in A_{A}$ e $\beta g=z_{2}$.

(iii) $z_{2}=\varepsilon q$.

Entäo, a aresta $\beta=(q, V, N, t) \in A_{A}$ e $B g=z_{2}$ 。

Mas, como $A \vec{e}$ deterninistica, nenhum dos casos acima pode ocorrer. $\log 0, z_{1}=u$ e wV $\in(\Gamma g) *$.

- Seja $u=q \varepsilon \in Q_{A} \varepsilon$.

A demonstação ë anäioga a anterior.

Assim, provamos que $g \vec{e}$ de atraso um da direita para a esquerda. De modo anälogo, podemos demonstrar que g é de atraso um da esquerda para a direita. Concluimos, então, que o morfismo g ê un côdigo de atraso um.

Demonstração da Proposicão $2 \mathrm{c}$

Queremos mostrar que $f$ é injetor sobre $\Gamma$. 
Como a måquina de Turing $A$ è reversîvel, segue que $A^{\prime}$ tambẻm è reversível; então, pela proposição 2 a o morfismo $g^{\prime}$ è injetor sobre $\Gamma^{\prime}$. Mas, como pela Proposição $1 \mathrm{f}=\phi g^{\prime} \psi$, com $\phi$ e $\psi$ morfismos bijetores, resulta que $f \vec{e}$ injetor sobre $\Gamma$.

Demonstração da Proposição $2 \mathrm{~d}$

Queremos mostrar que o morfismo $f \vec{e}$ um cödigo de atraso um.

Jâ que a måquina de Turing $A$ é reversível, segue que A' também é reversível; logo, pela Proposição $2 b$ o morfismo g' è un côdigo de atraso um.

Assim, pela Proposição 1 e por um resultado que apre sentamos em seguida, concluímos que fè um cỏdigo de atraso um .

$\underline{\text { PROPOSICÄO } 3}-$ Seja $g: \Gamma_{2}^{*} \longrightarrow \Gamma_{3}^{*}$ um cödigo de atraso um. Se jam dois morfismos bijetores $g_{1}: \Gamma_{1}^{*} \longrightarrow \Gamma_{2}^{*}$ e $g_{2}: \Gamma_{3}^{*} \longrightarrow \Gamma_{4}^{*}$. Então, o morfismo $g_{1} g g_{2}$ ê um cỏdigo de atraso um.

DEMONSTRACĨO - E suficiente mostrarmos que $g_{1} g$ e gg $g_{2}$ são de atraso um.

Vamos demonstrar inicialmente que $g_{1} g$ è de atraso um. Sejam $u$ e v em $\Gamma_{1} g_{1} g$ e w em $\Gamma_{3}^{*}$ tais que uvw $\epsilon\left(\Gamma_{1} g_{1} g\right)^{*}$. 
Como $g_{1}$ è sobrejetor segue que $\Gamma_{1} g_{1}=r_{2}$. Então, u e v pertencem a $\Gamma_{2} g$ e uvw $E\left(\Gamma_{2} g\right) *$

Mas, como g ê de atraso um da esquerda para a direita, concluimos que vw $\in\left(\Gamma_{2} g\right)^{*}=\left(r_{1} g g_{1} g\right)^{*}$.

Portanto, $g_{1} g$ è de atraso um da esquerda para a direi ta.

Analogamente, demonstra-se que $g_{1} g$ e de atraso um da direita para a esquerda.

Agora, demonstranos que $g_{2}$ è de atraso um.

Sejam $u$ e $v$ em $\Gamma_{2} g g_{2}$ e $w$ em $r_{4}^{*}$ tais que uviv $\in\left(r_{2} g g_{2}\right)^{*}$. Então, uvw $\in\left(\mathrm{r}_{2} \mathrm{~g}\right){ }^{*} \mathrm{~g}_{2}$.

Como $g_{2}$ è bijetor, segue que (uvw) $g_{2}^{-1} \in\left(r_{2} g\right)^{*} ;$ ou seja, $\left(\operatorname{ug}_{2}^{-1}\right)\left(\mathrm{vg}_{2}^{-1}\right)\left(\mathrm{wg}_{2}^{-1}\right) \in\left(\mathrm{\Gamma}_{2} \mathrm{~g}\right)^{*}$. Alêm disso, ug ${ }_{2}^{-1}$ e $\mathrm{vg}_{2}^{-1}$ pertencem a $\Gamma_{2} g$.

Mas, como g ë de atraso um da esquerda para a direita, concluimos que $\left(\mathrm{vg}_{2}^{-1}\right)\left(\mathrm{wg}_{2}^{-1}\right) \in\left(\mathrm{r}_{2} \mathrm{~g}\right)$, isto $\overrightarrow{\mathrm{e}},(\mathrm{Vw}) \mathrm{g}_{2}^{-1} \in\left(\mathrm{\Gamma}_{2} \mathrm{~g}\right)^{*}$. Logo, VW $E\left(\Gamma_{2} g g_{2}\right) *$

Assim, $\operatorname{gg}_{2}$ ê de atraso um da esquerda para a direita. Analogamente, demonstra-se que $\mathrm{gg}_{2}$ é de atraso um da direita para a esquerda.

3. CODIFICACR̃O DE UMA COMPUTACÃO

Nesta seção formalizamos um dos conceitos que será 
essencial para o restante deste trabalho.

Seja A uma māquina de Turing e sejam os conjuntos $\mathrm{CI}_{A}=\varepsilon \mathrm{T}_{A}^{*} \mathrm{Q}_{A} \mathrm{~T}_{A}^{*} \varepsilon$ e $\mathrm{Y}_{A}=\{1, \varepsilon\} \mathrm{T}_{A}^{*} \mathrm{~A}_{A} \mathrm{~T}_{A}^{*}\{1, \varepsilon\}$. Lembremos que ${ }^{\mathrm{CI}} \mathrm{A}$ $\vec{e}$ o conjunto das configurações instantâneas de $A$.

Para cada aresta $\alpha$ de $A, \alpha=(q, i, j, p)$, com $i \in E_{A}$ e $j \in D_{A}, \operatorname{seja}$

$$
\lceil\alpha\rfloor: \mathrm{CI}_{A} \longrightarrow \mathrm{Y}_{A}
$$

uma função parcial denominada funcão configurą̧̃o intermediäria associada à aresta $\alpha$.

Essa configuração intermediāria consiste em considerar, para cada uma das fitas de $A$, a configuração mais cur ta entre as configurações antes e depois de executar a aresta $\alpha$. Assim, para cada configuração instantânea u de A , $u=\varepsilon s q t \varepsilon, \operatorname{com} q \in Q_{A}$ e $s, t \in T_{A}^{*}$, de modo que a aresta a seja executâveI,

$$
u\lceil\alpha\rfloor=(\varepsilon s) l_{1} \alpha(t \varepsilon) \ell_{2}
$$

onde $\ell_{I}$ e $\iota_{2}$ são funções que dependem de $i$ e de $j$,

$$
\ell_{1}: \varepsilon T_{A}^{*} \longrightarrow\{1, \varepsilon\} T_{A}^{*} \quad \text { e } \quad \ell_{2}: T_{A}^{*} \in \longrightarrow T_{A}^{*}\{1, \varepsilon\}
$$

dadas por 


$$
(\varepsilon S) \ell_{1}=\left\{\begin{array}{l}
\varepsilon(s i), \quad \text { se } i=E_{\sigma}, \text { para algum } \sigma \text { em } T_{A} \\
1, \text { se } i=V \text { e } s=1 ; \\
\varepsilon S, \text { caso conträrio }
\end{array}\right.
$$

e

$$
(\tau \varepsilon) \ell_{2}=\left\{\begin{array}{l}
(\tau j) \varepsilon, \text { se } j=D_{\sigma}, \text { para algum o em } \mathrm{T}_{A} \\
1, \text { se } j=\mathrm{V} \text { e } t=1 \\
\tau \varepsilon, \\
\text { caso conträrio. }
\end{array}\right.
$$

Se a aresta a não for executävel sobre $u$, então $u\lceil\alpha\rfloor=\emptyset$.

A proposição que apresentamos a seguir mostra-nos que, se $f$ e $g$ são os morfismos associados a $A$, então a imagem por $f$ da configuração intermediâria associada a uma aresta a è a configuração instantânea após a execução de $\alpha$, ao passo que, por $g$, é a configuração instantânea antes da execução de $\alpha$.

PROPOSICAO 4 - Seja A uma mâquina de Turing serni-normalizadae sejam $f$ e g os morfismos associados a A. Então, para cada aresta $\alpha$ em $A_{A}$ e para cada configuração instantânea u em CI $I_{A}$ tal que a seja executâvel, são vâlidas as identidades:

(i) $\quad u\lceil a\rfloor g=u$

(ii) $u\lceil\alpha\rfloor f=\overline{u|| \alpha||}$. 
DEMONSTRACKO - Sejam A uma mäquina de Turing semi-normalizada e $f$ e $g$ os morfismos associados a $A$ e definidos como na Tabela 1 (päg.66).

Seja u em CI $A$, u=esqte, com q $\in Q_{A}, \quad s=\sigma_{1} \ldots \sigma_{k}, \sigma_{i} \in T_{A}$ $(1 \leq i \leq k)$ e $t=\tau_{1} \cdots \tau_{\ell}, \tau_{i} \in \mathrm{T}_{A}(I \leq i \leq \ell)$.

Examinamos cada uma das possibilidades para uma ares ta $\alpha$ em $A_{A}$ que seja executävel para $u$, a fim de demonstramos que $u\lceil\alpha\rfloor g=u$.

- Seja $\alpha=\left(q, E_{\gamma}, N, r\right)$, onde $\gamma=\sigma_{k}$.

$$
\text { Então, } \begin{aligned}
u\lceil\alpha . J & =\left(\varepsilon \sigma_{1} \cdots \sigma_{k-1}\left(q, E_{\gamma}, N, r\right) t \varepsilon\right) g \\
& =\varepsilon \sigma_{1} \cdots \sigma_{k-1} \sigma_{k} q t \varepsilon \\
& =\varepsilon s q t \varepsilon=u .
\end{aligned}
$$

- Seja $\alpha=\left(q, N, D_{\gamma}, r\right)$, onde $\gamma=\tau_{1}$.

A demonstração ê anäloga a anterior.

- Seja $\alpha=(q, V, N, r)$.

Nesse caso, $s=1$ e temos que

$$
\begin{aligned}
u\lceil\alpha\rfloor g & =((q, V, N, r) t \varepsilon) g \\
& =\varepsilon q t \varepsilon=u .
\end{aligned}
$$

- Seja $\alpha=(q, N, V, r)$.

Nesse caso, $t=1$ e a demonstração segue de modo anälogo a anterior. 
- Seja $\alpha=\left(q, N, D_{\gamma}^{-1}, r\right)$, para algum $\gamma$ em $T_{A}$. Então, $u\lceil\alpha\rfloor g=\left(\varepsilon s\left(q, N, D_{\gamma}^{-1}, r\right) t \varepsilon\right) g$

$$
=\varepsilon \mathrm{sqt} \varepsilon=\mathrm{u} \text {. }
$$

- Seja $\quad \alpha=\left(q, E_{\gamma}^{-1}, N, r\right)$, para algum $\gamma$ em $T_{A}$.

A demonstração é análoga a anterior.

- Seja $\alpha=(q, N, N, x)$.

Então, $u\lceil\alpha\rfloor g=\left(\varepsilon s(q, N, N, r) t_{\varepsilon}\right) g$

$$
=\varepsilon S q t \varepsilon=u \text {. }
$$

A demonstraçäo de que u[o.f $=\overline{u\|\alpha\|}$ segue pela. Pro posiçäo 1, pelo item (i) aplicado ao morfismo g' e pela Propo sição 2 (Capîtulo 2).

Na sequência, consideramos para cada aresta $\alpha$ em $A_{A}$, $\alpha=(q, i, j, p)$, com $i \in E_{A}$ e $j \in D_{A}$, uma função que a cada configura ção instantânea $u$ de $A$ tal que a aresta o seja executâvel, as socia uma codificação da computação da máquina A a partir da configuração instantânea u e segundo a aresta $\alpha$.

Vamos formalizar essa definição da função codificoşão da computação associada c̀ aresta a,

$$
[\alpha]: \mathrm{CI}_{A} \longrightarrow \mathrm{Y}_{A} \mathrm{~B} \overline{\mathrm{CI}}_{A}
$$

da seguinte forma. Para cada configuração instantânea u de A tal que a aresta a seja executảvel, 


$$
u[\alpha]=u[\alpha]+\overline{u|| \alpha||}
$$

caso contrário, $u[\alpha]=\emptyset$.

Podemos estender essa função para cada passeio não de generado $c$ em $A, c=\alpha_{1} \alpha_{2} \cdots \alpha_{m}, c o m m \geq 2$ e $\alpha_{k}$ em $A_{A}(1 \leq k \leq m)$, obtendo-se assim a função parcial

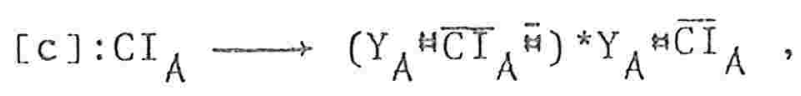

de modo que para cada configuração instantânea u de $A$ tal que - passeio c seja executảvel,

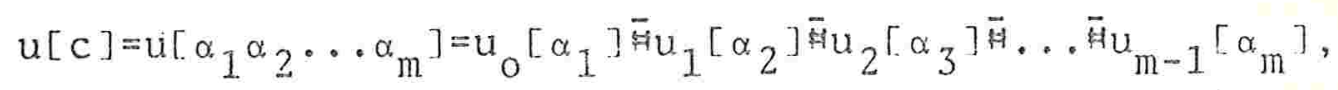

onde $u_{0}=u$ e para cada $k,(1 \leq k \leq m-1), u_{k}=u_{k-1}|| \alpha_{k}||$; caso contrâtio, $u[c]=\emptyset$.

Denominamos [c] de função codificasão da computação associada ao passeio c.

EXEMPLO 1 - Seja a måquina de Turing $A$ do Exemplo 1 (Capítulo 2) e consideremos a configuração instantânea $u=\varepsilon q_{0}$ abe de $A \quad e$ o passeio $c=\alpha_{1} \alpha_{2} \alpha_{3}$ em $A$, com $\alpha_{1}=\left(q_{0}, E_{a}^{-1}, D_{a}, q_{0}\right), \alpha_{2}=\left(q_{0}, E_{a}, D_{b}, q_{1}\right)$ e $\alpha_{3}=\left(q_{1}, v, v, q_{2}\right)$. Então, temos que

$$
u[c]=u\left[\alpha_{1} \alpha_{2} \alpha_{3}\right]=u_{0}\left[\alpha_{1}\right] \bar{s} u_{1}\left[\alpha_{2}\right] \bar{\psi} u_{2}\left[\alpha_{3}\right] .
$$

Mas, $\left.u_{0}\left[\alpha_{1}\right]=u_{0} \Gamma \alpha_{1}\right\rfloor \hat{u_{0}|| \alpha_{1}||}$

$$
=\left(\varepsilon q_{0} a b \varepsilon\right) \Gamma\left(q_{0}, E_{a}^{-1}, D_{a}, q_{0}\right) J \xi \overline{\left(\varepsilon q_{0} a b \varepsilon\right)\left\|\left(q_{0}, E_{a}^{-1}, p_{a}, q_{0}\right)\right\|}
$$




$$
\begin{aligned}
& =\varepsilon\left(q_{0}, E_{a}^{-1}, D_{a}, q_{0}\right) b \varepsilon+\bar{\varepsilon} \bar{a} \bar{q}_{0} \bar{b} \bar{\varepsilon} ; \\
& u_{1}\left[\alpha_{2}\right]=u_{1}\left\lceil\alpha_{2} \int \overline{u_{1}|| \alpha_{2}||}\right. \\
& \left.=\left(\varepsilon \mathrm{aq}_{0} \mathrm{~b} \varepsilon\right) \Gamma\left(\mathrm{q}_{0}, \mathrm{E}_{\mathrm{a}}, \mathrm{D}_{\mathrm{b}}, \mathrm{q}_{1}\right)\right\rfloor \overrightarrow{\left(\varepsilon a \mathrm{q}_{0} \mathrm{~b} \varepsilon\right)\left\|\left(\mathrm{q}_{0}, \mathrm{E}_{\mathrm{a}}, \mathrm{D}_{\mathrm{b}}, \mathrm{q}_{1}\right)\right\|} \\
& =\varepsilon\left(q_{0}, E_{a}, D_{b}, q_{1}\right) \varepsilon * \bar{\varepsilon} \bar{q}_{1} \bar{\varepsilon} \\
& \text { e } \quad u_{2}\left[\alpha_{3}\right]=u_{2}\left\lceil\alpha_{3}\right\rfloor \widehat{H u_{2}|| \alpha_{3}||} \\
& =\left(\varepsilon q_{1} \varepsilon\right) \Gamma\left(q_{1}, v, V, q_{2}\right) \text { 小 } \overline{\left(\varepsilon q_{1} \varepsilon\right)||\left(q_{1}, v, v, q_{2}\right) \|} \\
& =\left(q_{1}, V, V, q_{2}\right) 4 \bar{\varepsilon} \bar{q}_{2} \bar{\varepsilon}
\end{aligned}
$$

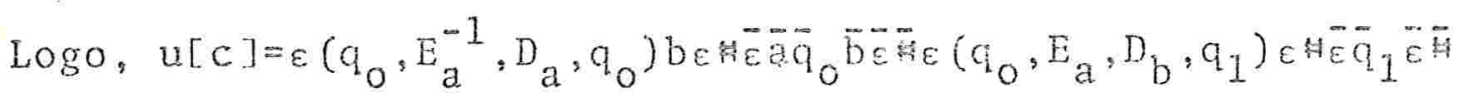

$$
\left(q_{1}, v, v, q_{2}\right)+\bar{\varepsilon} \overline{q_{2}} \bar{\varepsilon} \text {. }
$$

4. A INDECIDIBILIDADE DO PROBLEMA DE CORRESPONDENCIA DE POST PARA MORFISMOS QUE SÃO CODTGOS DE ATRASO UM

Nesta seçäo, o nosso objetivo é demonstrar o Teorema 2 que enunciamos no inicio deste capitulo e o faremos reduzin do o Problema da Pertinência para Linguagens Recursivamente $E$ numeräveis, que $\vec{e}$ conhecido sex indecidivel, para o problema de Correspondência de Post restrito a morfismos que são cödigos de atraso um. Essa redução ë obtida pelo seguinte lema. 
LEMA 1 - Para cada mäquina de Turing determinística $A$ e para cada palavra s em $\Sigma_{A}$, existem efetivamente alfabctos $\Gamma$ e $\Delta$ e morfismos $h_{1}$ e $h_{2}: r^{*} \longrightarrow \Delta^{*}$, ambos sendo cödigos de atraso um tais que

$$
s \in|A| \text { sse } w_{1}=w_{2} \text {, para algum } w \text { em } r^{+} \text {. }
$$

Para demonstrarmos o Lema 1 precisamos antes de alguns resultados auxiliares que apresentamos a seguir através de duas proposições.

PROPOSICÃO 5 - Seja A una mäquina de Turing semi-normalizada e sejam $f$ e g os morfismos associados a $A$. Para cada passeio não degenerado $c: q \rightarrow r$ em $A$ e para cada configuração ins tantânea $z=\varepsilon s q t \varepsilon$ de $A$, se $(\varepsilon s q t \varepsilon)|| c||=\varepsilon u r v \varepsilon$, para $u$ e $v$ em

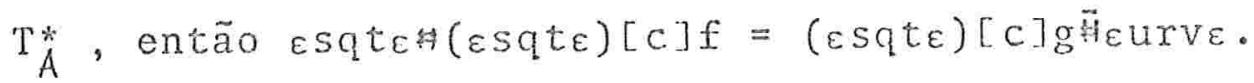

DEMONSTRACÃO - Sejam A uma mäquina de Turing semi-normalizada e $f$ e $g$ os morfismos associados a $A$.

Sejam c: $q \longrightarrow x$ um passeio não degenerado em $A$ e $z=\varepsilon s q t \varepsilon$ uma configuração instantânea de A tal que

$$
(\varepsilon \text { sqte })|| c||=\varepsilon \operatorname{urv} \varepsilon \text {, para } u \text { e } v \text { em } \mathrm{T}_{A}^{*} \text {. }
$$

Vamos provar por indução no comprimento do passeio c que

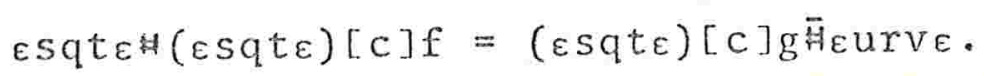


Inicialmente, suponhamos que c pertence a $A_{A}$. Entäo,

$$
(\varepsilon \mathrm{sqt} \varepsilon)[\mathrm{c}]=(\varepsilon \mathrm{Sq} t \varepsilon) \Gamma \mathrm{c} J \overline{(E S q t \varepsilon)|| c||}
$$

e, da Proposição 4 e de (2), obtemos que

$$
\begin{aligned}
& (\varepsilon s q t \varepsilon)[c] f=\overline{(\varepsilon q s t \varepsilon)|| c|| \bar{q}}(\varepsilon q s t \varepsilon)|| c|| \\
& =\bar{\varepsilon} \bar{u} \overline{\mathrm{V}} \bar{\varepsilon} \overline{a_{\varepsilon}} \operatorname{urv} \varepsilon \\
& \mathrm{e} \quad(\varepsilon s q t \varepsilon)[c] g=\varepsilon q S t \varepsilon H \overline{(\varepsilon q S t \varepsilon)\|c\|} \\
& =\varepsilon q \operatorname{qs} \varepsilon \bar{\varepsilon} \bar{u} \vec{T} \vec{v} \text {. }
\end{aligned}
$$

Logo, a igualdade (3) $\ddot{e}$ văjida.

Suponhamos agora que c seja um passeio de comprinento $k \geq 2, \quad c=a_{1} \alpha_{2} \ldots \alpha_{k}, \operatorname{com} \alpha_{i} \in A_{A}(1 \leq i \leq k)$ Seja $d=\alpha_{1} \cdots \alpha_{k-1}$ e $p$ o tëmino do passeio d. Então, de (2) segue que existem pala vras $x$ e $y$ em $T_{A}^{*}$ tais que

$$
\begin{gathered}
(\varepsilon s q t \varepsilon)|| d||=\varepsilon x p y \varepsilon \\
\text { e } \quad(\varepsilon \operatorname{xpy} \varepsilon)|| \alpha_{k}||=\text { eurve. }
\end{gathered}
$$

Logo, pela hipōtese da indução aplicada ao passeio d, obtemos que

$$
\varepsilon \operatorname{sqt\varepsilon } H(\varepsilon \operatorname{sqt} \varepsilon)[\mathrm{d}] f=(\varepsilon s q \tau \varepsilon)[\mathrm{d}] \mathrm{g} \overline{\tilde{H}} \varepsilon \operatorname{xpy} \varepsilon \text {. }
$$

Assim, utilizando (4), (5) e a Proposição 4, obtemos que 


$$
\begin{aligned}
& \text { Esqten(esqte)「c]f= } \\
& =\varepsilon \operatorname{sqt\varepsilon } \varepsilon^{H}\left((\varepsilon \operatorname{sqt} \varepsilon)\lceil\mathrm{d}\rceil \bar{H}(\varepsilon \times p y \varepsilon)\left\lceil\alpha_{k}\right]\right) f
\end{aligned}
$$

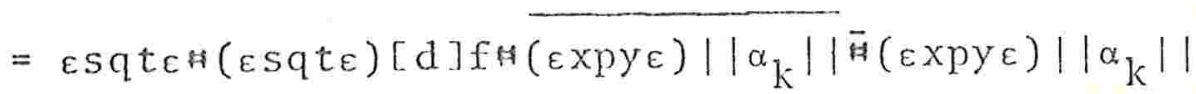

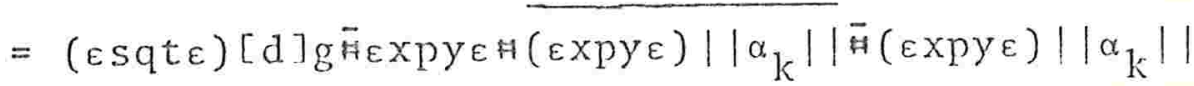

$$
\begin{aligned}
& =\left((\varepsilon \operatorname{sq} t \varepsilon)[d] \bar{q}(\varepsilon \times p y \varepsilon)\left[\alpha_{k}\right]\right) g \bar{F}(\varepsilon x p y \varepsilon)|| \alpha_{k} ! \mid \\
& =(\varepsilon \operatorname{sqt} \varepsilon)[\mathrm{c}] \mathrm{g} \overline{\mathrm{q}} \varepsilon \mathrm{urv} \varepsilon \text {. }
\end{aligned}
$$

Ou seja, a igualdade (3) è väIida.

Desse modo, concluĩmos a demonstração da proposição

5.

PROPOSICÃO 6 - Sejam A uma mäquina de Turing semi-normalizada e $f$ e $g: \Gamma^{*} \longrightarrow \Delta^{*}$ os moxfismos associados a $A$, com $\Delta=X U \bar{X}$. Sejam ainda os morfismos

$$
\underline{b}: \Delta^{*} \longrightarrow X^{*} \text {, definido por } \delta \underline{b}=\bar{\delta} \underline{b}=\delta \text {, para cada } \delta \text { em } x
$$$$
\text { e } \quad h_{A_{A}}: \Gamma^{*} \longrightarrow A_{A}^{*} \text {, que preserva } A_{A} \text {. }
$$

Se existem uma configuração instantânea u de A e palavras w em $\Gamma^{*}$ e $V$ em $X^{+}$tais que

$$
u H(w \underline{b})=(w g \underline{b}) v,
$$

então existem palavras $s$ e $t$ em $(X \backslash\{a\}) *$ tais que

$$
\text { (i) } v=s+4 \text {, }
$$


(ii) ts é uma configuração instantânea de A

e (iii) existe um passejo $c$ em $A$ com $c=w h_{A_{A}}$, se ${ }^{w h} \Lambda_{A} \neq 1$; caso conträrio, $c=1$, onde $q \vec{e}$ o estado do controle finito de A quando na configuração instantânea $u$. Alëm disso, $u|| c||=$ ts.

DEMONSTRACÃO - Seja A uma mãquina de Turing semi-normalizada. Seja $X=T_{A} U\{\varepsilon, H\} \cup Q_{A}$ e sejam os alfabetos $\Gamma=T_{A} \cup\{\varepsilon, H\} \cup A_{A} \cup \bar{X}$ e $\Delta=X U \tilde{X}$.

Consideremos $f$ e $g: \Gamma^{*} \longrightarrow \Delta^{*}$ os morfismos associados a $A$.

Sejam u uma configuração instantânea de $A$ e as palavras $w$ em $\Gamma^{*}$ e $v$ em $X^{*}$ tais que

$$
u f(w f \underline{b})=(w g \underline{b}) v .
$$

Demonstraremos, por indução no comprimento $n$ de $w$, que existem palavras $s$ e $t$ en $(X \backslash\{\dot{q}\})^{*}$ tais que as condiçöes (i), (ii) e (iii) estejam verificadas.

No decorrer desta demonstração, escrevemos simples mente h para o morisisno $h_{A_{A}}$.

Se $n=0$ então $w=1$ e. (6) fica reduzido a uH $=v$. Logo, considerando $s=u$ e $t=1$, as condições (i) (ii) e (iii) ficam trivialmente verificadas.

Suponhamos entäo que a proposição seja vâlida para as palavras $w$ de comprimento $n>0$ e vamos provar para uma pala vra $w$ de comprimento $n+1$. 
Assim, seja $w=\gamma_{1} \gamma_{2} \cdots \gamma_{n+1}$, com $\gamma_{i}$ em $r(1 \leq i \leq n+1)$. De (6) segue que

$$
u *\left(\gamma_{1} \gamma_{2} \ldots \gamma_{n+1}\right) f \underline{b}=\left(\gamma_{1} \gamma_{2} \cdots \gamma_{n+1}\right) g \underline{b v}_{n+1},
$$

com $v_{n+1}=v$

Por outro lado, pelas definiçōes de $f$ e g, também existe alguma palavra $v_{n}$ em $X^{+}$tal que

$$
u H\left(\gamma_{1} \gamma_{2} \cdots \gamma_{n}\right) \underline{f b}=\left(\gamma_{1} \gamma_{2} \cdots \gamma_{n}\right) g \underline{b v}_{n} .
$$

Então, pela hipótese da indução, existem palavras $s_{n}$ e $t_{n}$ em $(X \backslash\{A\}) *$ tais que

(i) $\quad v_{n}=s_{n} n t_{n}$,

(ii) $t_{n} s_{n}$ ê uma configuração instantânea de $A$

e

(iii) existe um passeio $c_{n}$ em $A$, com $c_{n}=\left(\gamma_{1} \gamma_{2} \cdots \gamma_{n}\right) h$, se $\left(\gamma_{1} \gamma_{2} \ldots \gamma_{n}\right) h \neq 1$;

caso concrärio $c_{n}=1_{q}$, onde $q \vec{e}$ o es tado do controle finito de A quando na configuração instantânea $u$. Alẻm disso, $u|| c_{n}||=t_{n} s_{n}$.

Mas, de (7) e (8) resulta que

$$
v_{n}\left(\gamma_{n+1} \underline{f b}\right)=\left(\gamma_{n+1} g \underline{b}\right) v_{n+1}
$$


e, utilizando (9) vem que

$$
s_{n} H t_{n}\left(\gamma_{n+1} f \underline{b}\right)=\left(\gamma_{n+1} g b\right) v_{n+1} \cdot
$$

A seguir, investigamos as värias possibilidades para. $r_{n+1}$ em $\mathrm{r}$

Seja $\gamma_{n+1}$ en $\Gamma \backslash\{H, \overline{\#}\}$. Então, $\gamma_{n+1} g \underline{b}$ e $\gamma_{n+1} f \underline{b} \in$ $(X \backslash\{H\})^{+}$. Logo, de (12) segue que

$$
s_{n}=\left(\gamma_{n+1} g \underline{b}\right) s_{n+1} \text {, para algum } s_{n+1} \text { em }(X \backslash\{a\}) *
$$

e considerando $t_{n+1}=t_{n}\left(\gamma_{n+1} \underline{f b}\right)$, temos que $t_{n+1} \in(X \backslash\{*\}) *$.

Entäo, utilizando (8), (9) e (13), obtemos que

$$
\begin{aligned}
& u a\left(\gamma_{1} \ldots \gamma_{n} \gamma_{n+1}\right) f \underline{b}=u f\left(\gamma_{1} \ldots \gamma_{n}\right) \underline{f b}\left(\gamma_{n+1} \underline{f b}\right) \\
& =\left(\gamma_{1} \ldots \gamma_{n}\right) g b v_{n}\left(\gamma_{n+1} f b\right) \\
& =\left(\gamma_{1} \ldots \gamma_{n}\right) g \underline{b s}_{n}+t_{n}\left(\gamma_{n+1} \underline{E b}\right) \\
& =\left(\gamma_{1} \ldots \gamma_{n}\right) g \underline{b}\left(\gamma_{n+1} g \underline{b}\right) s_{n+1} t_{n+1} \\
& =\left(\gamma_{1} \cdots \gamma_{n+1}\right) g \underline{b} s_{n+1}^{H t} t_{n+1} .
\end{aligned}
$$

Assim, de (7) segue que a condição (i) estä verificada, ou se ja, $v_{n+1}=s_{n+1}+t_{n+1}$.

A seguir, vamos provar que 
(ii) $t_{n+1}^{s} n+1$ è configuração instantânea de $A$ (14)

e (iii) existe um passeio $c_{n+1}$ em $A, \operatorname{com} c_{n+1}=w h$, se whł1; caso conträrio, $c_{n+1}=I_{q}$, onde $q$ é o estado de controle finito de $A$ quando na configuração instantânea u. Alëm disso, $u|| c_{n+1}||=t_{n+1} s_{n+1}$.

Inicialmente, suponhamos que $\gamma_{n+1} \in \Gamma \backslash\left(\Lambda_{A} \cup\{\dot{H}, \bar{H}\}\right)$. Nesse caso, é fácil ver que $\gamma_{n+1} \underline{g} \underline{b}=\gamma_{n+1} \underline{f b}$ e que $\gamma_{n+1} h=1$. Logo, verificamos (14), pois utilizando (10) e (13) obtemos que

$$
t_{n+1} s_{n+1}=t_{n}\left(\gamma_{n+1} f b\right) s_{n+1}=t_{n}\left(\gamma_{n+1} g b\right) s_{I n+1}=t_{n} s_{n}
$$

E, como $\left(\gamma_{1} \ldots \gamma_{n} \gamma_{n+1}\right) h=\left(\gamma_{1} \ldots \gamma_{n}\right) h$, resulta de (11) que o passeio $c_{n+1}=c_{n}$ satisfaz (15). De fato, se whýl então $\left(\gamma_{1} \ldots \gamma_{n}\right) h t 1$ e, consequentemente,

$$
c_{n+1}=c_{n}=\left(\gamma_{1} \ldots \gamma_{n}\right) h=w h
$$

se wh=1 então $\left(\gamma_{1} \ldots \gamma_{n}\right) h=1$ e $c_{n+1}=c_{n}=1$. Alêm disso, temos que

$$
u|| c_{n+1}||=u|| c_{n}||=t_{n} s_{n}=t_{n+1} s_{n+1} .
$$

Agora, vamos examinar cada uma das possibilidades pa ra $\gamma_{n+1}$ em $A_{A}$, onde $q$ e p denotam elementos de $Q_{A}$ e a é um sim bolo em $\mathrm{T}_{A}$. 
- Seja $\gamma_{n+1}=\left(q, E_{\sigma}, N, p\right)$. Então,

$$
\gamma_{n+1} \underline{g b}=\sigma q \quad \text { e } \quad \gamma_{n+1} \underline{f b}=p .
$$

Logo, utilizando (13) obtemos que

$$
\begin{gathered}
t_{n} s_{n}=t_{n}\left(\gamma_{n+1} g \underline{b}\right) s_{n+1}=t_{n} \sigma q s_{n+1} \\
\text { e } \quad t_{n+1} s_{n+1}=t_{n}\left(\gamma_{n+1} \underline{f b} s_{n+1}=t_{n} p s_{n+1} .\right.
\end{gathered}
$$

- Seja $\gamma_{n+1}=\left(q, N, D_{\sigma}, p\right)$.

A demonstração ẻ análoga a anterior.

- Seja $\gamma_{n+1}=(q, V, N, p)$. Então.

$$
\gamma_{n+1} g \underline{b}=\varepsilon q \quad \text { e } \quad \gamma_{n+1} \underline{f b}=\varepsilon p \text {. }
$$

Logo, de (13) temos que

$$
t_{n} s_{n}=t_{n}\left(\gamma_{n+1} g \underline{b}\right) s_{n+1}=t_{n} \varepsilon q s_{n+1}
$$

e $\quad t_{n+1} s_{n+1}=t_{n}\left(\gamma_{n+1} \underline{f b} s_{n+1}=t_{n} \varepsilon p s_{n+1}\right.$.

Mas, pela hipótese da indução, $t_{n} s_{n}$ è uma configuração ins tantânea de $A$; então $t_{n}=1$.

- Seja $\gamma_{n+1}=(q, N, V, p)$.

A demonstração é análoga a anterior.

- Seja $\gamma_{n+1}=\left(q, E_{G}^{-1}, N, p\right)$. Então,

$$
\gamma_{n+1} g \underline{b}=q \quad \text { e } \quad \gamma_{n+1} \underline{f b}=\sigma p .
$$


E, utilizando (13) segue que

$$
\begin{gathered}
t_{n} s_{n}=t_{n}\left(\gamma_{n+1} \underline{g}\right) s_{n+1}=t_{n} q s_{n+1} \\
e \quad t_{n+1} s_{n+1}=t_{n}\left(\gamma_{n+1} \underline{f}\right) s_{n+1}=t_{n} \sigma p s_{n+1} .
\end{gathered}
$$

- Seja $\gamma_{n+1}=\left(q, N, D_{\sigma}^{-1}, p\right)$

A demonstração ê anảloga a anterior.

- Seja $\gamma_{n+1}=(q, N, N, p)$. Então,

$$
\gamma_{n+1} g \underline{b}=q \quad \text { e } \quad \gamma_{n+1} \underline{f b}=p
$$

Logo, de (13) resulta que

$$
\begin{gathered}
t_{n} s_{n}=t_{n}\left(\gamma_{n+1} g \underline{b}\right) s_{n+1}=t_{n} q s_{n+1} \\
e \quad t_{n+1} s_{n+1}=t_{n}\left(\gamma_{n+1} \underline{\underline{b}}\right) s_{n+1}=t_{n} p s_{n+1} .
\end{gathered}
$$

Mas, em cada um dos casos que acabamos de considerar temos que

$$
t_{n+1} s_{n+1}=\left(t_{n} s_{n}\right)|| r_{n+1}|| \text { e } r_{n+1} h=r_{n+1} \text {. }
$$

Assim, segue de (11) que o passeio $c_{n+1}=c_{n}{ }_{n+1}$ è tal que se $\left(\gamma_{1} \ldots \gamma_{n}\right) h=1$ então $c_{n+1}=1 \gamma_{n+1}=\gamma_{n+1}=\left(\gamma_{1} \ldots \gamma_{n+1}\right) h=w h \quad$ e se $\left(\gamma_{1} \ldots \gamma_{n}\right) h \neq 1$ então $c_{n+1}=\left(\gamma_{1} \ldots \gamma_{n}\right) h \gamma_{n+1}=$ wh. $\quad$ Alëm disso, $u|| c_{n+1}||=u|| c_{n}|||| \gamma_{n+1}||=\left(t_{n} s_{n}\right)|| r_{n+1}||=t_{n+1} s_{n+1}$. Portan to, (14) e (15) estão satisfeitas para $s_{n+1}$ e $t_{n+1}$ obitidas em (13). 
Finalmente, suponhamos que $\gamma_{n+1} \in\{H, \bar{H}\}$. Então, $\gamma_{n+1} g \underline{b}=\gamma_{n+1} f \underline{b}=H$.

Logo, resulta de (12) que $\mathrm{s}_{\mathrm{n}}=1$. E, considerando $s_{n+1}=t_{n}$ e $t_{n+1}=I$ segue que $s_{n+1}$ e $t_{n+1} \in(X \backslash\{H\}) *$.

Entāo, utilizando (8), (9) e (16) obtemos que

$$
\begin{aligned}
& u *\left(\gamma_{1} \ldots \gamma_{n+1}\right) f \underline{b}=u *\left(\gamma_{1} \ldots \gamma_{n}\right) f \underline{b}\left(\gamma_{n+1} f \underline{b}\right) \\
& =\left(\gamma_{1} \ldots \gamma_{n}\right) g \underline{b} v_{n}\left(\gamma_{n+1} \underline{f b}\right) \\
& =\left(\gamma_{1} \cdots \gamma_{n}\right) g \underline{b} s_{n}+4 t_{n}\left(\gamma_{n+1} \underline{f b}\right)
\end{aligned}
$$

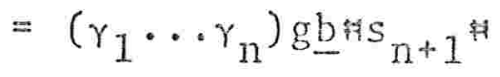

$$
\begin{aligned}
& =\left(\gamma_{1} \ldots \gamma_{n} \gamma_{n+1}\right) g b s_{n+1} * t_{n+1} \text {. }
\end{aligned}
$$

Assim, segue de (7) que $v_{n+1}=s_{n+1} h_{n+1}$ e a condição (i) estä verificada.

$$
\begin{aligned}
& \text { Alëm disso, resulta de (16) que } \\
& t_{n+1} s_{n+1}=s_{n+1}=t_{n}=t_{n} s_{n} \text {, verificando }(14) .
\end{aligned}
$$

E segue de (11) que o passeio $c_{n+1}=c_{n}$ satisfaz (15), ja que wh $=\left(\gamma_{1} \ldots \gamma_{n}\right) h$.

Desse modo, finalizamos a demonstração da Proposição 6.

Estamos agora em condições de demonstrar o Lema 1 , que enunciamos novamente. 
LEMA 1 - Para cada mäquina de Turing determinística $A$ e para cada palavra $s$ em $\Sigma_{A}^{*}$, existem efetivamente alfabetos $\Gamma$ e $\Delta$ e côdigos $h_{1}, h_{2}: \Gamma^{*} \longrightarrow \Delta^{*}$, de atraso um, tais que

$s \in|A|$ sse $w_{1}=w_{2}$, para algum $w$ em $r^{+}$.

DEMONSTRACÃO - Seja A uma mäquina de Turing deterministica e seja s uma palavra em $\Sigma_{A}^{*}$.

Pelo Teorema 1 (Capítulo 2) existe uma mäquina de Tú ring reversivel e normalizada $B$ tal que $|A|=|B|$.

Seja $B^{\prime}$ a reversa de $B$ e seja $C$ o acoplamento de $B$ com $B^{\prime}$. Lembremos que a mäquina de Turing $C$ tambëm ê reversí vel e normalizada.

Então, pela Proposição 2 resulta que os morfismos $f$ e g associados a c são códigos de atraso um.

Sejam $\Gamma_{1}$ e $\Delta_{1}$ os alfabetos tais que $f, g: r_{1}^{*} \rightarrow \Delta *$.

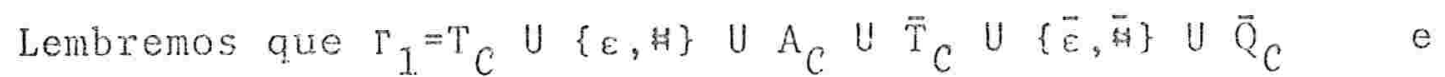
$\Delta_{1}=\mathrm{T}_{\mathrm{C}} \cup\{\varepsilon, H\} \cup \mathrm{Q}_{\mathrm{C}} \cup \overline{\mathrm{T}}_{\mathrm{C}} \cup\{\vec{\varepsilon}, \bar{A}\} \cup \overrightarrow{\mathrm{Q}}_{\mathrm{C}}$.

Consideremos então os alfabetos

$$
\Gamma=\Gamma_{1} U\{r,-1\} \text { e } \Delta=\Delta_{1} U\{r,-1\}
$$

e os morfismos $h_{1}, h_{2}: \Gamma^{*} \longrightarrow \Delta^{*}$ definidos pela tabeta 5 , onde $\left\{q_{-}\right\}=I_{C}=I_{B}$ 


\begin{tabular}{|c|c|c|}
\hline$\gamma \in \Gamma$ & $\gamma h_{1}$ & $\gamma h_{2}$ \\
\hline$r$ & $r \varepsilon q_{-} s \varepsilon q$ & $r$ \\
$r$ & -1 & $\bar{\theta}_{\varepsilon q_{-}^{\prime}} s_{\varepsilon-1}$ \\
$\gamma_{1} \in \Gamma_{1}$ & $\gamma_{1} \mathrm{f}$ & $\gamma_{1} g$ \\
\hline
\end{tabular}

Tabeza 5 - Definição dos morfismos $h_{1}$ e $h_{2}$.

Pelo fato dos morfismos $f$ e g serem cödigos de atraso um e ainda dos simbolos $r$ e $\rightarrow$ näo pertencerem a $\Gamma_{1}$ nem a $\Delta_{1}$, resulta que os morfismos $h_{1}$ e $h_{2}$ são cödigos de atraso um.

Podemos observar que o morfismo $h_{1}$ depende da palava. $s$ que representa a entrada para a mäquina de Turing $B$, mas $h_{2}$ não depende do conteûdo da fita à esquerda no instante en que $B$, eventualmente, pära com a entrada $s$. Ou seja, $h_{1}$ e $h_{2}$ dependem apenas de $s$, porque estamos considerando a máquina de Turing $c$, onde $(1, s)$ ë a configuração injicial e final de suas fitas, caso $s \in|A|=|B|$; daj a importância dessa mâquina $C$, que ê acoplamento de $B$ com $B^{\prime}$.

$$
\text { Resta-nos mostrar então que } s \in|A| \text { sse } w_{1}=w h_{2} \text {, pa }
$$
ra algum em $\mathrm{r}^{+}$.

Inicialmente, suponhamos que $s \in|A|$. Como $|A|=|B|$, então $s \in|B|$. Mas, $C$ è o acoplamento de $B \operatorname{com} B^{\prime}$; então pela Proposição 4 (Capîtulo 2) segue que $C$ tem um passeio $c: q_{-} \longrightarrow q_{-}^{\prime}$, onde $\left\{q_{-}\right\}=I_{C}=I_{B}$, tal que 


$$
(\varepsilon q-s \varepsilon)|| c||=\varepsilon q \leq s \varepsilon .
$$

Logo, pela proposição 5 resulta que

$$
\varepsilon q_{\ldots} s \varepsilon f\left(\varepsilon q_{-} s \varepsilon\right)[c] f=\left(\varepsilon q_{-} s \varepsilon\right)[c] g \overline{H_{1}} \varepsilon q_{-}^{\prime} s \varepsilon \text {. }
$$

Então,

$$
\vdash \varepsilon q_{-} s \varepsilon H\left(\varepsilon q_{-} s \varepsilon\right)[c] f \dashv=\vdash\left(\varepsilon q_{\ldots} s \varepsilon\right)[c] g \bar{H} \varepsilon q_{-}^{\prime} s \varepsilon-1 \text {. }
$$

Mas, pelas definições dos morfismos $h_{1}$ e $h_{2}$ temos que

$$
\begin{aligned}
& r \varepsilon q_{-} s \varepsilon d=r h_{1} \text {, } \\
& \left(\varepsilon q_{.} s \varepsilon\right)[c] f=\left(\varepsilon q_{-} s \varepsilon\right)[c] h_{1} \\
& \text { e } \quad+=-1 h_{1} \text {; } \\
& r=r h_{2}, \\
& \left(\varepsilon q_{-} s \varepsilon\right)[c] g=\left(\varepsilon q_{\ldots} s \varepsilon\right)[c] h_{2} \\
& \text { e मีEq:se-1 }=-h_{2} \text {. }
\end{aligned}
$$

Portanto,

$$
\left(r-\left(\varepsilon q_{-} s \varepsilon\right)[c]-1\right) h_{1}=\left(1-\left(\varepsilon q_{-} s \varepsilon\right)[c]-1\right) h_{2} \cdot
$$

Assim, existe $w=r\left(\varepsilon q_{-} s \varepsilon\right][c] \dashv$ em $r^{+}$tal que wh ${ }_{1}=w_{2}$ *

Reciprocamente, suponhamos que $w_{1}=w_{2}$, para algum $w$ em $\Gamma^{+}$. Observamos inicialmente que para cada $\gamma$ em $\Gamma, \gamma h_{1}{ }^{t} \gamma h_{2}$; então $|w| \geq 2$. 
Alêm disso, $\vdash(-1)$ è o ünico elemento de $\Gamma$ tal que $\vdash h_{1}$ e $t_{2}\left(-h_{1} e+h_{2}\right)$ são segmentos iniciais (finais) de uma mes-

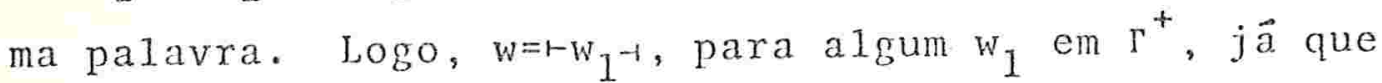
$(1--1) h_{1} \frac{1}{F}(1--1) h_{2}$

Podemos supor ainda, sem perda de generalidade, que nenhum dos dois simbolos, $t$ ou $t$, ocorre em $w_{1}$.

De fato, suponhamos que $t$ ocorra em $w_{1}$; então $w_{1}$ tem uma fatoração $w_{1}=w_{2}+w_{3}$, onde + não ocorre em $w_{2}$. Is to implica que

$$
\left(1-w_{2}-1 w_{3}{ }^{-1}\right) h_{1}=\left(-w_{2}-1 w_{3}^{-1}\right) h_{2}
$$

ou seja,

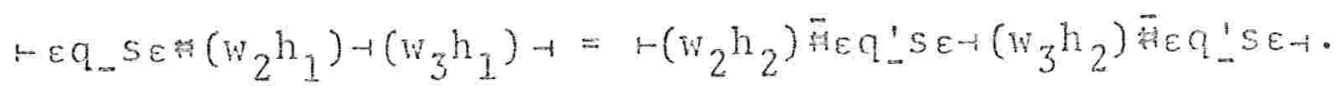

Mas, como t näo ocorre em $\mathrm{w}_{2}$, segue que t não ocorre nem em $w_{2} h_{1}$ nem en $w_{2} h_{2} \cdot \operatorname{Logo}$,

$$
r \varepsilon q_{-} \operatorname{sen}\left(w_{2} h_{1}\right)-t=r\left(w_{2} h_{2}\right) \bar{H} \varepsilon q_{-}^{\prime} s \varepsilon-1
$$

Portanto, $\left(1-w_{2}-1\right) h_{1}=\left(1-w_{2}-1\right) h_{2} e+$ não ocorre em $w_{2}$.

Analogamente, se $r$ ocorre em $w_{2}$, então $w_{2}$ tem um seg mento final $w_{4}$ tal que nem $r$ e nem tocorre em $w_{4}$ e $\left(1-w_{4}^{-1}\right) h_{1}=$ $=\left(1-w_{4}^{-1}\right) h_{2}$.

Assim, podemos supor que nenhum dos dois simbolos, $r$ ou $t$, ocorre em $w_{1}$ (isto é, $w_{1} \in \mathrm{I}_{1}^{+}$). 


$$
\begin{aligned}
& \text { Então, de }\left(1-w_{1}^{-1}\right) h_{1}=\left(1-w_{1}^{-1}\right) h_{2} \text { segue que } \\
& r \varepsilon q_{-} s \varepsilon *\left(w_{1} h_{1}\right)^{-1}=r\left(w_{1} h_{2}\right) \bar{H} \varepsilon q_{-}^{\prime} s \varepsilon-1 .
\end{aligned}
$$

Logo,

$$
\varepsilon q_{-} s \varepsilon H\left(w_{1} f\right)=w_{I} g \bar{f} \in q_{-}^{\prime} s \varepsilon \text {. }
$$

E, pela Proposição 6 resulta que existe um passeio c em c tal que

$$
\left(\varepsilon q_{-} s \varepsilon\right)|| c||=\varepsilon q_{-}^{\prime} s \varepsilon
$$

Como $C \vec{e}$ o acoplamento de $B$ com $B^{\prime}$, obtemos pela proposição 4 (Capitulo 2) que $s \in|B|$. Mas, $|B|=|A|$. Portanto, s $6|A|$. Assim, completamos a demonstraçäo do Lema 1.

Finalmente, vamos demonstrar o Teorema 2 que represen ta o resultado principal deste capitulo.

TEOREMA 2 - o problema de Correspondência de post è indecidí.vel mesmo quando restrito a cödigos de atraso um.

DEMONSTRACAO - Suponhamos que o Problema de Correspondência de Post para co̊digos de atraso um seja decidỉvel.

Sejam A uma mâquina de Turing determinística e s uma palavra em $\Sigma_{A}^{*}$

Pelo Lema 1 , existem alfabetos $\Gamma$ e $\Delta$ e morfismos $\mathrm{h}_{1}$ e $h_{2}: \Gamma^{*} \longrightarrow \Delta^{*}$, ambos sendo cỏdigos de atraso um tais que $s \in$ $|A|$ sse $w_{1}=w_{2}$, para algum w em $r^{+}$. 
Alêm disso, os morfismos $h_{1}$ e $h_{2}$ podem ser obtidos $\underline{e}$ fetivamente a partir de $A$ e de $s$.

Então, existe um algoritmo para determinar se uma pa lavra pertence ou não a uma linguagem reconhecida por uma mäquina de Turing determinística dada. O que è um absurdo, pois - Problema da Pertinência para Linguagens Recursivamente Enumerâveis è indecidjuel.

Assim, concluimos que o problema de Correspondência de Post restrito a côdigos de atraso um é indecidível.

5. A INDECTDTBILIDADE DO PROBLEMA DE CORRESPONDENCTA DE POST PARA CODIGOS DE ATRASO UM PERTENCENTES A PS I SP

Nesta seção fortalecemos o Teorema 2 , obtendo o resul tado que enunciamos a seguir.

TEOREMA 3 - O Problema de Correspondência de Post permanece in decidivel mesmo quando restrito a cödigos de atraso um perten centes a PSNSP.

Para demonsträ-10 precisamos fortalecer também outros resultados jä vistos.

Inicialmente, vamos mostrar que os morfismos construi dos na Proposição 2 pertencem a PSISP, ou seja: 


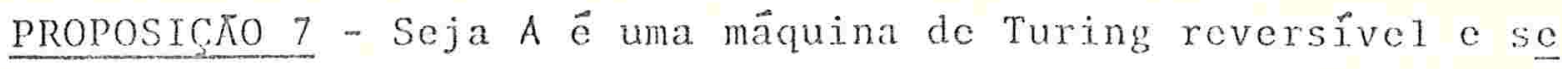
mi-normalizada. Entäo, ambos os morfismos associados a $A$ são cödigos de atraso um e pertencem a PSASP.

DEMONSTRACATO - E suficiente mostraxmos que os morfismos $f \mathrm{c} g$ construídos na demonstração da Proposição 2 pertencem a PSnSP.

Os morfismos $f, g: \Gamma^{*} \longrightarrow \Delta^{*}$,

onde $\quad \Gamma=T_{A} \cup\{\varepsilon, n\} \cup A_{A} \cup \bar{T}_{A} \cup\{\bar{\varepsilon}, \bar{i}\} \cup \bar{Q}_{A}$

e $\Delta=T_{A} \cup\{\varepsilon, \beta\} \cup Q_{A} \cup \bar{T}_{A} \cup\{\bar{\varepsilon}, \bar{B}\} \cup \bar{Q}_{A}$,

estão definidos pela Tabela 1 (päg.66).

Com a finalidade de mostrarmos que $g$ E PS, considere mos o alfabeto

$$
\theta_{1}=I_{A} \cup\{\varepsilon, 4\} \cup Q_{1} \cup K_{I} \cup K_{2} \cup \tilde{T}_{A} \cup\{\bar{\varepsilon}, \bar{a}\} \cup \bar{Q}_{A},
$$

onde $Q_{1}=\left\{q_{Q} \in Q_{A} /\right.$ näo existem en $A$ arestas com origem em q e rön tulo $\mathrm{E}_{\sigma} \times \mathrm{N}$, para algum o em $\mathrm{T}_{\mathrm{A}}$ ou $\left.\mathrm{V} \times \mathrm{N}\right\}$,

$K_{I}=\left\{(\sigma, q) \in T_{A} \times Q_{A} /\right.$ existem em A arestas com origem em $\mathrm{q}$ e rötulo $\left.\mathrm{E}_{\sigma} \times \mathrm{N}\right\}$

e $\quad K_{2}=\left\{(\varepsilon, q) \in \varepsilon \times Q_{A} /\right.$ existem em A arestas com origen em q e rótulo $V \times N\}$.

Sejam os morfismos

$$
p_{I}: \Gamma^{*} \longrightarrow \theta_{1}^{*} \quad \text { e } \quad s_{I}: \theta_{1}^{*} \longrightarrow \Delta^{*},
$$


definidos pela Tabeza 6 , onde o denota um símbolo em $\mathrm{T}_{\mathrm{A}} ; \mathrm{q} \mathrm{e}$ $r$ são elementos de $Q_{A} ;(q, i, j, r)$, com $i \in E_{A}$ e $j \in D_{A}$, representa uma aresta em $A_{A}$ e $(\sigma, q)$ e $(\varepsilon, q)$ pertencem a $K_{1}$ e $K_{2}$, respectivamente.

E fäcil verificar que o morfismo $p_{1}\left(s_{1}\right)$ é um prefixo (sufixo) e que $g=p_{1} s_{1}$.

\begin{tabular}{|c|c|}
\hline$\gamma \in \Gamma$ & $\gamma p_{1}$ \\
\hline$\sigma$ & $\sigma$ \\
$\bar{\sigma}$ & $\bar{\sigma}$ \\
$\varepsilon$ & $\varepsilon$ \\
$\bar{\varepsilon}$ & $\bar{\varepsilon}$ \\
$q$ & 4 \\
$\bar{H}$ & $\bar{q}$ \\
$(q, E, N, r)$ & $(\sigma, q)$ \\
$(q, N, D, r)$ & $q \sigma$ \\
$(q, V, N, r)$ & $(\varepsilon, q)$ \\
$(q, N, V, r)$ & $q \varepsilon$ \\
$\left(q, E_{\sigma}^{-1}, N, r\right)$ & $q$ \\
$\left(q, N, D_{\sigma}^{-1}, r\right)$ & $q$ \\
$(q, N, N, r)$ & $q$ \\
$\bar{q}$ & $\bar{q}$ \\
\hline
\end{tabular}

\begin{tabular}{|c|c|}
\hline$\theta \in \theta_{1}$ & $\theta s_{1}$ \\
\hline$\sigma$ & $\sigma$ \\
$\bar{\sigma}$ & $\bar{\sigma}$ \\
$\varepsilon$ & $\varepsilon$ \\
$\bar{\varepsilon}$ & $\bar{\varepsilon}$ \\
$q$ & $\bar{q}$ \\
$\bar{k}$ & $\bar{q}$ \\
$q \in Q_{1}$ & $q$ \\
$(\sigma, q)$ & $\sigma q$ \\
$(\varepsilon, q)$ & $\varepsilon q$ \\
$\bar{q}$ & $\bar{q}$ \\
\hline
\end{tabular}

Tabela 6 - Definição dos morfismos $\mathrm{p}_{1}$ e $\mathrm{s}_{1}$ associados a g. 
A fim de mostrarmos que g tambêm pertence a $S P$, consideremos o alfabeto

$$
\theta_{2}=\mathrm{T}_{\mathrm{A}} \cup\{\varepsilon, H\} \cup Q_{2} \cup K_{3} \cup K_{4} \cup \overline{\mathrm{T}}_{A} \cup\{\bar{\varepsilon}, \overline{4}\} \cup \bar{Q}_{A} \text {, }
$$

onde $Q_{2}=\left\{q \in Q_{A} /\right.$ näo existem em $A$ arestas com origem em $q$ e rö tulo $N \times \mathrm{D}_{\sigma}$, para algum $\sigma \mathrm{em}_{\mathrm{A}}$ ou $\mathrm{N} \times \mathrm{V}$, $K_{3}=\left\{(q, \sigma) \in Q_{A} \times T_{A} /\right.$ existem en A arestas com origem em q e rötulo $\left.N \times D_{\sigma}\right\}$

e $\quad K_{4}=\left\{(q, \varepsilon) \in Q_{A} x \varepsilon /\right.$ existem em A arestas com origem em q e rótulo $N \times V\}$.

Sejam os morfismos

$$
s_{2}: I^{*} \quad \rightarrow \theta_{2}^{*} \quad \text { e } \quad p_{2}: \theta_{2}^{*} \longrightarrow \Delta^{*}
$$

definidos pela Tabeza 7 , onde o denota un simbolo em $\mathrm{T}_{A}$; $\mathrm{q}$ e $x$ são elementos de $Q_{A} ;(q, i, j, r)$, com $i \in E_{A}$ e $j \in D_{A}$, representa uma aresta en $A_{A}$ e $(q, \sigma)$ e $(q, \varepsilon)$ portencem a $K_{3}$ e $K_{4}$, respectivamente.

Pode-se verificar tambëm que o morfismo $p_{2}\left(s_{2}\right) \ddot{e}$ um prefixo (sufixo) e que $g=\mathrm{s}_{2} \mathrm{p}_{2}$.

Desse modo, concluímos que g pertence a PSחSP.

Precisamos demonstrar ainda que o morfismo f tambëm pertence a PSnSP. Mas, como A é reversivel segue que A' tambëm ë reversivel e o morfismo g' associado a $A^{\prime}$ pertence a PSISP (como acabamos de mostrar). 
Assim, pela proposição 1 e pelas proposições 8 e 9 que apresentamos a seguir, concluimos que f pertence a PSnSP.

\begin{tabular}{|c|c|}
\hline$\gamma \in \Gamma$ & $\gamma s_{2}$ \\
\hline$\sigma$ & $\sigma$ \\
\hline $\bar{\sigma}$ & $\bar{\sigma}$ \\
\hline$\varepsilon$ & $\varepsilon$ \\
\hline $\bar{\varepsilon}$ & $\bar{\varepsilon}$ \\
\hline H & 的 \\
\hline 特 & $\overline{4}$ \\
\hline$\left(q, E_{\sigma}, N, r\right)$ & oq \\
\hline$\left(q, N, D_{\sigma}, r\right)$ & $(q ; \sigma)$ \\
\hline$(q, V, N, r)$ & $\varepsilon q$ \\
\hline$(q, N, v, r)$ & $(q, \varepsilon)$ \\
\hline$\left(q, E_{\sigma}^{-1}, N, r\right)$ & $\mathrm{q}$ \\
\hline$\left(q, N, D_{\sigma}^{-1}, x\right)$ & $\mathrm{q}$ \\
\hline$(q, N, N, r)$ & $q$ \\
\hline $\bar{q}$ & $\bar{q}$ \\
\hline
\end{tabular}

\begin{tabular}{|c|c|}
\hline$\theta \in \theta_{2}$ & $\theta p_{2}$ \\
\hline$\sigma$ & $\sigma$ \\
$\varepsilon$ & $\bar{\sigma}$ \\
$\bar{\varepsilon}$ & $\varepsilon$ \\
$\beta$ & $\bar{\varepsilon}$ \\
$\bar{q}$ & $\dot{q}$ \\
$q \in Q_{2}$ & $q$ \\
$(q, \sigma)$ & $q \sigma$ \\
$(q, \varepsilon)$ & $q \varepsilon$ \\
$\bar{q}$ & $\vec{q}$ \\
\hline
\end{tabular}

Tabeza 7 - Definjção dos morfismos $\mathrm{s}_{2}$ e $\mathrm{p}_{2}$ associados a $g$. 
PROPOSTCKO 8 - Seja $\mathrm{f}: \mathrm{r}_{1}^{*} \longrightarrow \mathrm{r}_{2}^{*}$ um morfismo bijetor e seja $h: \Gamma_{2}^{*} \longrightarrow \Gamma_{3}^{*}$ um prefixo (sufixo). Então, fh è um prefixo (sufixo).

DEMONSTRACRO - Sejam $x$ e y em $r_{1}$ tais que

$$
x f h=(y f h) z \quad(x f h=z(y f h)) \text {, para algum z em } \Gamma_{3}^{*} \text {. }
$$

Entล̃o,

$$
(x f) h=(y f) h z \quad((x f) h=z(y f) h) .
$$

Como h ë um prefixo (sufixo) e paxa cada $\gamma$ em $\Gamma_{1}$, yf $\in \Gamma_{2}$, se gue que $x f=y f$ e $z=1$.

$$
\begin{aligned}
& \text { Mas, } f \vec{e} \text { injetor, logo, } x=y . \\
& \text { Portanto, the è um prefjxo (sufixo). }
\end{aligned}
$$

PROPOSICR̃ 9 - Seja h: $\Gamma_{1}^{*} \rightarrow \Gamma_{2}^{*}$ um prefixo (sufixo) e seja $f: \Gamma_{2}^{*} \longrightarrow \Gamma_{3}^{*}$ um morfismo bijetor. Então, hf è un prefixo (sufi $\mathrm{xo}$ )

DEMONSTRACAO - Sejam $x$ e $y$ em $r_{1}$ tais que

$$
x h f=(y h f) z \quad(x h f=z(y h f)) \text {, para algum z em } \Gamma_{3}^{\circ}
$$

Como f è bijetor, podemos escrever

$$
(x h) f=\left((y h)\left(z f^{-1}\right)\right) f \quad\left((x h) f=\left(\left(z f^{-1}\right)(y h)\right) f\right)
$$


ou seja,

$$
x h=(y h)\left(z f^{-1}\right) \quad\left(x h=\left(z f^{-1}\right)(y h)\right) .
$$

Mas, como h $\vec{e}$ um prefixo (sufixo) segue que $x=y$ e $z f^{-1}=1$. Logo, $x=y$ e $z=1$.

Portanto, hf è um prefixo (sufixo).

Podemos demonstrar tambêm que os morfismos $h_{1}$ e $h_{2}$, dados pelo Lema 1 , pertencem a PSnSP. Obtemos assim o seguin te resuitado.

LEMA 2 - Para cada mäquina de Turing determinística A e para cada palavra $s$ em $\Sigma_{A}^{*}$, existem efetivamente alfabetos $\Gamma$ e $\Delta$ e morfismos $h_{1}, h_{2}: r^{*} \rightarrow \Delta^{*}$, que são cödigos de atraso um e per tencen a PSISP, tais que

$$
\text { sE }|A| \text { sse } \mathrm{Wh}_{1}=\mathrm{wh}_{2} \text {, para algun } \mathrm{w} \text { em } \mathrm{r}^{+} \text {. }
$$

DEMONSTRACAO - Basta mostramos que os morfismos $h_{1}$ e $h_{2}$, cons truỉdos na demonstração do Lema l, pertencem a PSnSP.

Como foi visto, os morfismos $h_{1}$ e $h_{2}$ säo extensōes, respectivamente, dos morfismos $f$ e g associados à mäquina de Turing $C$ que é o acoplamento de $B \operatorname{com} B^{\prime}$, onde $B$ e a mâquin a de Turing reversivel e normalizada equivalente a $A$.

os morfismos $f$ e g estão definidos en $\Gamma_{1}^{*}$ com valores em $\Delta \stackrel{\dot{i}}{\text {, onde }}$

$$
\Gamma_{1}=T_{C} \cup\{\varepsilon, \mu\} \cup A_{C} \cup \bar{T}_{C} \cup\{\bar{\varepsilon}, \bar{A}\} \cup \bar{Q}_{C}
$$


e

$$
\Delta_{1}=T_{C} \cup\{\varepsilon, *\} \cup Q_{C} \cup \vec{T}_{C} \cup\{\bar{E}, \bar{H}\} \cup \bar{Q}_{C} .
$$

Pela Proposição 7 segue que os morfismos $f$ e g perten cem a PSnSP. Nesse caso, existem alfabetos $\theta_{1}, \theta_{2}, \theta_{3}$ e $\theta_{4}$, mor fismos prefixos

$$
\mathrm{p}_{1}: \Gamma_{1}^{*} \longrightarrow \theta_{1}^{*}, \mathrm{p}_{2}: \theta_{2}^{*} \longrightarrow \Delta_{1}^{*}, \mathrm{p}_{3}: \Gamma_{1}^{*} \longrightarrow \theta_{3}^{*} \text { e } \mathrm{p}_{4}: \theta_{4}^{*} \longrightarrow \Delta_{1}^{*}
$$

e morfismos sufixos

$$
s_{1}: \theta_{1}^{*} \longrightarrow \Delta_{1}^{*}, s_{2}: \Gamma_{I}^{*} \longrightarrow \theta_{2}^{*}: s_{3}: \theta_{3}^{*} \longrightarrow \Delta_{1}^{*} \text { e } s_{4}: \Gamma_{1}^{*} \longrightarrow \theta_{4}^{*}
$$

tais que $f=p_{1} s_{1}=s_{2} p_{2}$ e $g=p_{3} s_{3}=s_{4} p_{4}$

Os morfismos $h_{1}$ e $h_{2}$ estäo definidos em $r^{*}$ com valores em $\Delta^{*}$, onde

$$
\Gamma=\Gamma_{1} U\{1,-1\} \quad \text { e } \quad \Delta=\Delta_{1} U\{r,-1\} \text {. }
$$

Consideremos entäo os alfabetos

$$
\Xi_{i}=\theta_{i} U\{1,-1\} \text {, para cada } i, 1 \leq i \leq 4
$$

e os morfismos

$$
\begin{aligned}
& \phi_{1}: \Gamma^{*} \longrightarrow \Xi_{1}^{*}, \psi_{1}: \Xi_{1}^{*} \longrightarrow \Delta^{*}, \\
& \psi_{2}: \Gamma^{*} \longrightarrow \Xi_{2}^{*}, \phi_{2}: \Xi_{2}^{*} \longrightarrow \Delta^{*}, \\
& \phi_{3}: \Gamma^{*} \longrightarrow \Xi_{3}^{*}, \psi_{3}: \Xi_{3}^{*} \longrightarrow \Delta^{*}, \\
& \text { e } \psi_{4}: \Gamma^{*} \longrightarrow \Xi_{4}^{*}, \phi_{4}: \Xi_{4}^{*} \longrightarrow \Delta^{*},
\end{aligned}
$$

definidos pelas Tabelas 8 a 12 . 


\begin{tabular}{|c|c|c|c|c|}
\hline$\gamma \in \Gamma$ & $\gamma^{\phi_{1}}$ & $\gamma \psi_{2}$ & $\gamma \phi_{3}$ & $Y \psi_{4}$ \\
\hline$\vdash$ & $r \varepsilon q_{-} s \varepsilon$ & $\vdash$ & $r$ & r \\
\hline-1 & -1 & $\dashv$ & -1 & $\bar{H} \in q_{-}^{\prime} s \varepsilon \dashv$ \\
\hline$\gamma_{1} \in \Gamma_{1}$ & $r_{1} p_{1}$ & $\gamma_{1} s_{2}$ & $\gamma_{1}{ }^{\rho}{ }_{3}$ & $\gamma_{1} s_{4}$ \\
\hline
\end{tabular}

Tabela 8 - Definição dos morfismos $\phi_{1}, \psi_{2}, \phi_{3}$ e $\psi_{4}$.

\begin{tabular}{|ccc|c|}
\hline$\theta$ & $G$ & $\Xi_{1}$ & $\theta \psi_{1}$ \\
\hline 1 & & - \\
-1 & & -1 \\
$\theta_{1}$ & $\in$ & $\theta_{1}$ & $\theta_{1} s_{1}$ \\
\hline
\end{tabular}

Tabeza 9 - Definiçäo do norfismo 1

\begin{tabular}{|ccc|c|}
\hline$\theta$ & $\in$ & $\Xi_{3}$ & $\theta \psi_{3}$ \\
\hline$r$ & & $r$ \\
-1 & & $\bar{\mu}_{\varepsilon q_{-}^{\prime}} \mathrm{s} \varepsilon-1$ \\
$\theta_{3}$ & $\in$ & $\theta_{3}$ & $\theta_{3} s_{3}$ \\
\hline
\end{tabular}

Tabela 11 - Definição do morfismo $\psi_{3}$.

\begin{tabular}{|ccc|c|}
\hline$\theta$ & $\epsilon$ & $\Xi_{2}$ & $\theta_{2}$ \\
\hline$r$ & & $1-\varepsilon q_{2} s \varepsilon \dot{ }$ \\
$\dashv$ & & -1 \\
$\theta_{2}$ & $\epsilon$ & $\theta_{2}$ & ${ }_{2} p_{2}$ \\
\hline
\end{tabular}

Tabeza 10 - Definição do morfismo $\phi_{2}$.

\begin{tabular}{|ccc|c|}
\hline$\theta$ & $\epsilon$ & $\Xi_{4}$ & $\theta_{4}$ \\
\hline+ & & & 1 \\
-1 & & & -1 \\
$\theta_{4}$ & $\in$ & $\theta_{4}$ & $\theta_{4} p_{4}$ \\
\hline
\end{tabular}

Tabela 12 - Definiçãa do morfismo $\phi_{4}$. 
Pode-se verificar que os morfismos $\phi_{1}, \phi_{2}, \phi_{3}$ e $\phi_{4}$ $\left(\psi_{1}, \psi_{2}, \psi_{3}\right.$ e $\psi_{4}$ ) são prefixos (sufixos) c que $h_{1}=\phi_{1} \psi_{1}=\psi_{2} \phi_{2}$ e $\mathrm{h}_{2}=\phi_{3} \psi_{3}=\psi_{4} \phi_{4}$. Portanto, $\mathrm{h}_{1}$ e $h_{2}$ pertencem a PSISP.

\section{Demonstracão do Teorema 3}

É anäloga à demonstração do Teorema 2, utilizando-se - Lema 2 no lugar do Lema 1.

6. A CONDICAO DE OUTRAS RESTRICOES DO PROBLEMA DE CORRESPONDENCTA DE POST

O problema de Correspondência de post quando un dos morfismos ê um prefixo foi mostrado ser indecidivel por Cousi neau (comunicaçäo pessoa1) equando ambos os norfismos säo pre-fixos tambêm foi provado que $\ddot{e}$ indecidivel por Cousineau e Vi dal-Naquet (comunicação pessoa1). Por dualidade, o resultado segue para morfismos sufixos.

porëm, outxas questöes ainda permanecem em aberto,co mo por exemplo, o Problema de Correspondência de post quando restrito a morfismos biprefixos ou quando os morfismos são cle mentares.

Por outro lado, o Problema de Correspondência de Post foi mostrado ser decidivel em mais ciois casos. 
TEOREMA 4 - Karhumaki e Simon (1979).

O Problema de Correspondência de Post ê decidível quando um dos morfismos ë periódico.

Obs.: Dizemos que um morfismo $h: \Lambda^{*} \longrightarrow B^{*}$ é periödico se $A h \subseteq z^{*}$ para alguma palavra $z \mathrm{em} \mathrm{B*}$.

TEOREMA 5 - Ehrenfeucht, Karhumaki e Rozenberg (1982).

O Problema de Correspondência de Post com listas consistindo de duas palavras $\vec{e}$ decidivel. 


\section{CAPITULO 4}

\section{REPRESENTACÃO DE LINGUAGENS}

\section{RECURSTVAMENTE ENUMERAVEIS}

A finalidade deste capitulo é apresentar un dos resultados principais deste trabalho que $\vec{e}$ a representação de uma linguagem recursivamente enumerável como a imagem homomörfica de um conjunto-igualdade minimal de dois cödigos $h_{1}$ e $h_{2}$, sendo que $h_{1} \vec{e}$ de atraso um da esquerda para a direita e $h_{2}$ é de atraso um da direita para a esquerda. Em seguida, ve-rificamos que $h_{1}$ e $h_{2}$ pertencem a $P S$.

Além disso, apresentamos tambëm outras representações de linguagens recursivamente enumeráveis. 
1. UMA REPRESIENTACXO DE IIINGUAGINS RIECURSTVAMENTE ENUMER VEIS ATRAVTS DE CODTGOS DI: ATRASO UM IEM SENTIDOS OPOSTOS

Recentemente, várias representações de linguagens recursivamente enumeráveis, utilizando conjuntos-igualdade de morfismos de monóides livres, têm sido apresentadas. Um primeiro resultado neste sentido é o de Salomaa (1978).

\section{TEOREMA 1 - Salomaa}

Para cada linguagem recursivamente enumerável $L \subseteq \Sigma^{*}$, existem efetivamente morfismos $h_{1}, h_{2}: \Gamma^{*} \longrightarrow \Delta^{*}$ e uma função $g: \Gamma^{*} \rightarrow \Sigma^{*}$ computada por uma đgsm (mäquina sequencial generalizada deter ministica) tajs que $L=\left(E_{q}\left(h_{1}, h_{2}\right)\right) 8$.

Culik (1979b) fortaleceu essa representaçäo, mostran do que a dgsm g pode ser restrita a um morfismo $h_{\Sigma}$ que preser va $\Sigma$ se o conjunto-igualdade de $h_{1}$ e $h_{2}$ for substituido pelo conjunto-igualdade minimal de $h_{1}$ e $h_{2}$.

\section{TEOREMA 2 - Culik}

Para cada linguagem recursivamente enumerävel $\mathrm{L} \subseteq \Sigma^{*}$, existem efetivamente um morfismo $h_{\Sigma}: \Gamma^{*} \longrightarrow \Sigma^{*}$, que preserva $\Sigma$ e dois morfismos $h_{1}, h_{2}: \Gamma^{*} \longrightarrow \Delta *$ tais que $L=\left(\right.$ eq $\left.\left(h_{1}, h_{2}\right)\right) h_{\Sigma}$.

Apresentamos a scguir uma idéia geral da demonstração feita por Culik.

São construídos dois morfismos $h_{1}$ e $h_{2}: \Gamma^{*} \rightarrow \Delta^{*}$ tais 
que uma palavra w em $\Gamma^{*}$ pertence a eq $\left(h_{1}, h_{2}\right)$ sse w è uma codi ficação de uma derivação de alguma palavra $\mathrm{s}$ em $\mathrm{L} \subseteq \sum^{*}$. Então, aplica-sc o morfismo $h_{\Sigma} a w$, obtendo-sc s.

Podemos notar a diferença fundamental de $\mathrm{h}_{1} \mathrm{e} \mathrm{h}_{2}$ com relação aos morfismos construídos para a demonstração da inde cidibilidade do Problema de Correspondência de Post (Lema 1 Capítulo 3):

$h_{1}$ e $h_{2}$ são independentes de qualquer palavra $s$ em $L$.

Seja $G=(N, \Sigma, S, P)$ uma gramätica do tipo 0 tal que $L=L(G)$, onde $N$ è o conjunto de não-ierminais, $\Sigma$ é o conjunto de terminais, $P \subseteq(N \cup \Sigma){ }^{*} N(N \cup \Sigma) * \times(N \cup \Sigma) * \bar{e}$ o conjunto de produções e $S \in N$ é o símbolo inicial.

Para que toda palavra em L(G) tenha uma derivação de comprimento impar, supomos que $\mathrm{P}$ tenha a produção $(S, S)$.

$$
\begin{aligned}
\text { Sejam } & =\{H\} \cup N \cup \sum U\{\langle\alpha, \beta\rangle /(\alpha, \beta) \in P\}, \\
\bar{E} & =\{\bar{\sigma} / \sigma \in \Xi\} \text { e } \hat{\Sigma}=\{\hat{\sigma} / \sigma \in \Sigma\} .
\end{aligned}
$$

Considere os alfabetos

$$
\Gamma=\Xi U \equiv U \hat{\Sigma} U\{r, \$, a, c, d\}
$$

e

$$
\Delta=\Xi U \bar{\Xi} U \quad\{\mathrm{a}, \mathrm{b}, \mathrm{c}, \mathrm{d}, \vdash, \dashv\}
$$

e dois morfismos $h_{1}, h_{2}: \Gamma^{*} \longrightarrow \Delta^{*}$ definidos pela Tabela 1. 


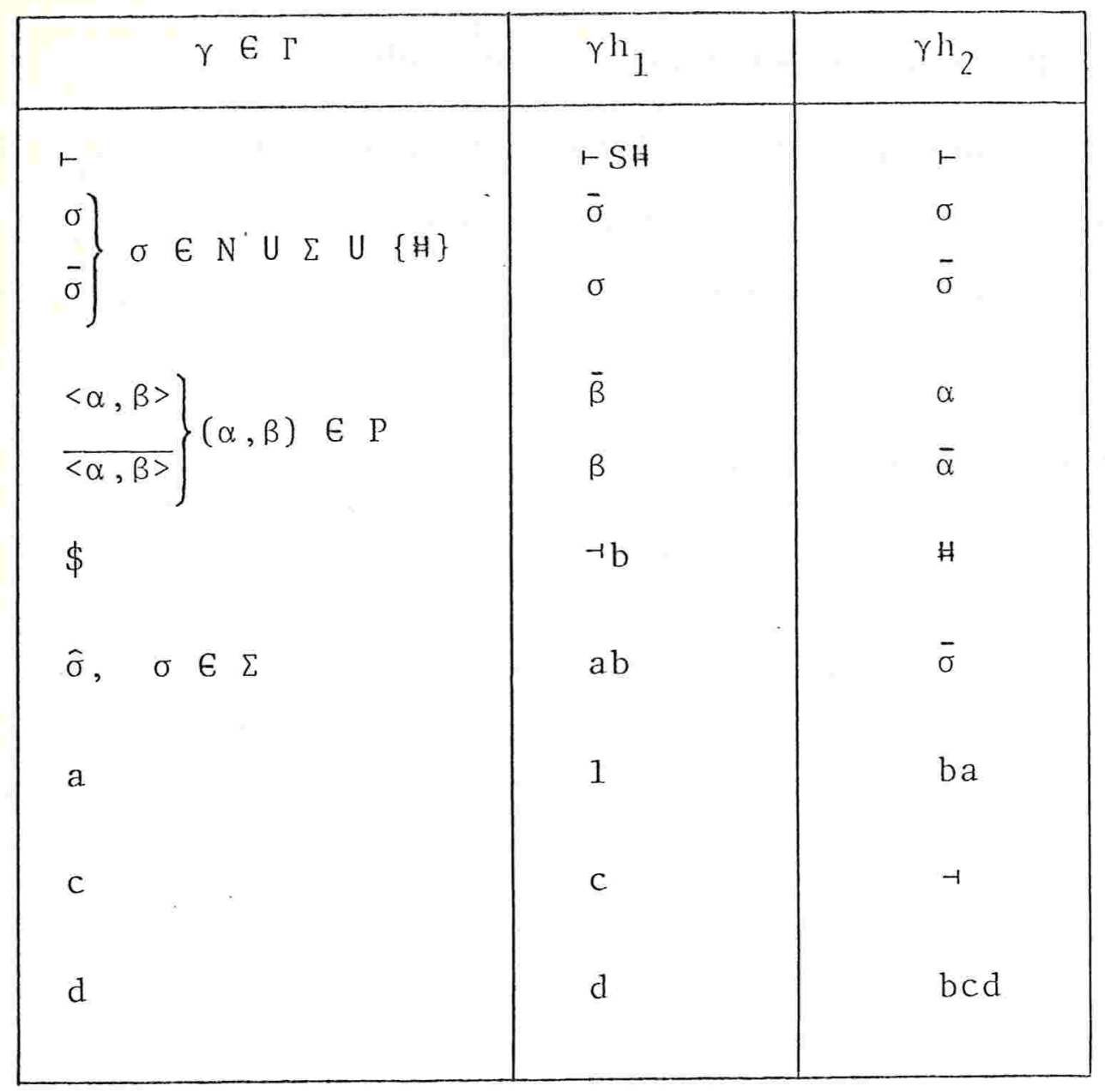

Tabela 1 - Definição dos morfismos $\mathrm{h}_{1}$ e $\mathrm{h}_{2}$

Seja o morfismo $h_{0}: \Gamma^{*} \longrightarrow \Sigma *$ definido por $\hat{\sigma} \mathrm{h}_{0}=\sigma$, para cada $\sigma$ em $\Sigma$

e $\quad \sigma h_{0}=1$, para cada $\sigma$ em $\Gamma \backslash \hat{\Sigma}$.

Se s $€ L$, a gramática $G$ tem uma derivação de comprimento $\mathrm{n}$ para $\mathrm{s}$, com $\mathrm{n}$ ímpar,

$$
\mathrm{S}=\mathrm{s}_{0} \underset{\mathrm{G}}{\Longrightarrow} \mathrm{s}_{1} \underset{\mathrm{G}}{\Longrightarrow} \mathrm{s}_{2} \underset{\mathrm{G}}{\Longrightarrow} \ldots \mathrm{s}_{\mathrm{n}}=\mathrm{s}
$$


onde, para cada $i(0 \leq i \leq n-1), s_{i} \Longrightarrow s_{i+1}$ é obtida usando uma produção $\mathrm{p}_{\mathrm{k}_{i}}=\left(\alpha_{i}, \beta_{i}\right)$ de $\mathrm{p}=\left\{\mathrm{p}_{1}, \mathrm{p}_{2}, \ldots, \mathrm{p}_{\mathrm{m}}\right\}$.

$$
\begin{aligned}
& \text { Então, existem } u_{i} \text { e } v_{i} \text { tais que } \\
& s_{i}=u_{i} \alpha_{i} v_{i} \quad(0 \leq i \leq n-1) \\
& \text { e } \quad s_{i+1}=u_{i} \beta_{i} v_{i}(0 \leq i \leq n-1) \text {. } \\
& w=r<S, \beta_{0}>A \bar{u}_{1}<\overline{\alpha_{1}, \beta_{1}}>\bar{v}_{1} \bar{H}_{2}<\alpha \alpha_{2}, \beta_{2}>v_{2} H_{\bar{u}} \bar{u}_{3}<\overline{\alpha_{3}}, \beta_{3}>\bar{v}_{3} \bar{H} \ldots \\
& \ldots \nexists u_{n-1}<\alpha_{n-1}, \beta_{n-1}>v_{n-1} \$ \hat{s}_{n} c a^{\left|s_{n}\right|} d \text {. }
\end{aligned}
$$

Portanto, como mostrado na Figura 1 temos que $w_{1}=w_{2}$ e para cada $u$ em $\Gamma^{+}$, se $w=u v$, para $v$ em $\Gamma^{+}$, então uh $_{1} \neq$ uh. $_{2}$.

Alëm disso, wh ${ }_{0}=s$. Assim, $L \subseteq\left(\mathrm{eq}\left(\mathrm{h}_{1}, \mathrm{~h}_{2}\right)\right) \mathrm{h}_{0}$.

Reciprocamente, se $w \in$ eq $\left(h_{1}, h_{2}\right)$ então w deve ser da forma $w=r z \hat{s} c a|s| d$, para algum $z$ em $(\Xi U \bar{\Xi}){ }^{*}$ e $s$ em $\Sigma^{*}$ tal que $\mathrm{S} \underset{\mathrm{G}}{\Longrightarrow} \mathrm{s} . \quad$ Logo, $\left(\mathrm{eq}\left(\mathrm{h}_{1}, \mathrm{~h}_{2}\right)\right) \mathrm{h}_{0} \subseteq \mathrm{L}_{\text {. }}$.

$\mathrm{Na}$ demonstração acima, trocando-se $\Sigma$ por $\hat{\Sigma}$ e vice-versa, podemos utilizar o morfismo $h_{\Sigma}$ do enunciado, ao invés do morfismo $\mathrm{h}_{0}$.

Dado o resultado de Lecerf quanto à indecidibilidade do Problema de Correspondência de Post para morfismos inje tores, era natural investigar se o Teorema 2 continuaria vảlí. 


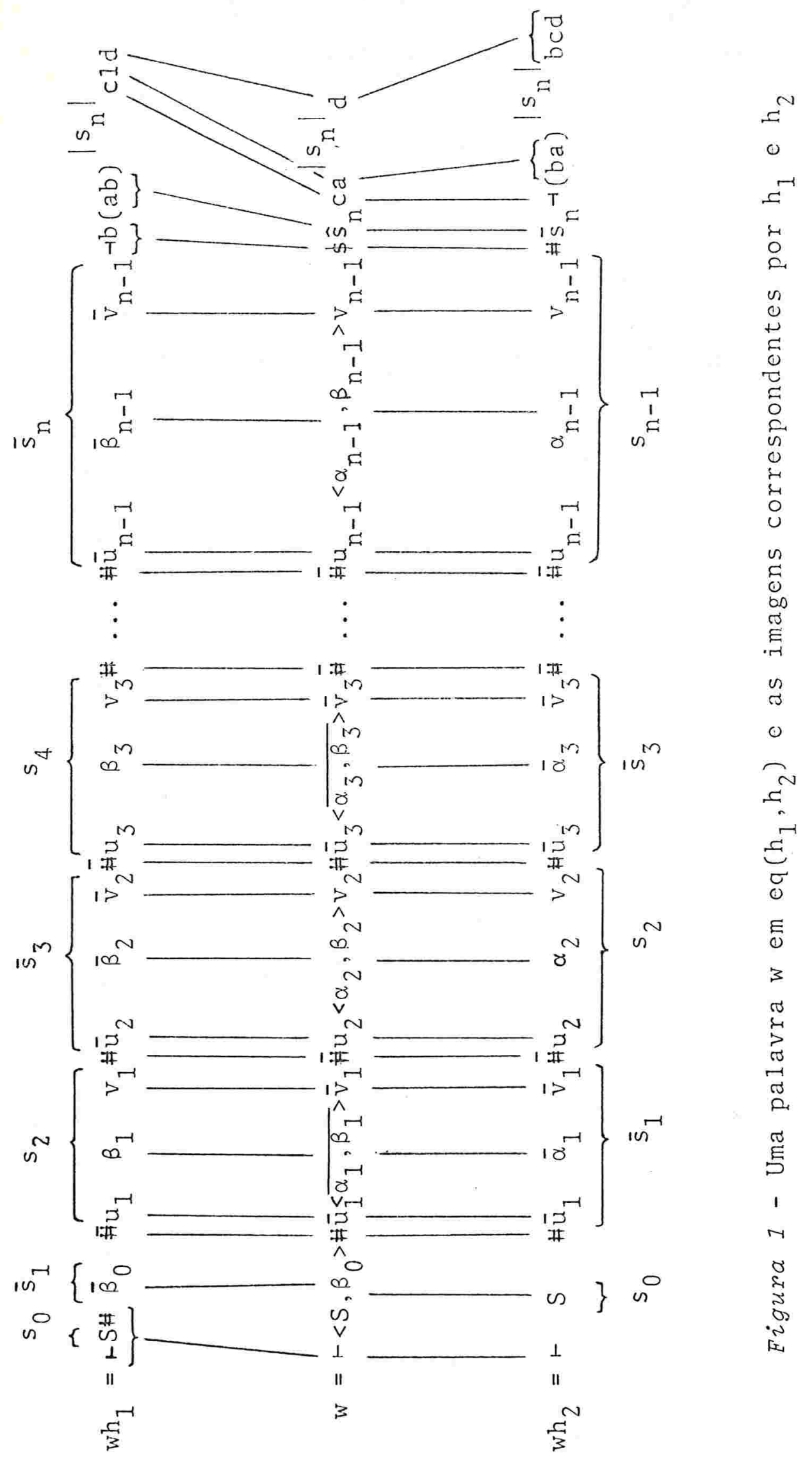


do mesmo quando $h_{1}$ e $h_{2}$ fossem injetores. Ou seja,

"para cada linguagem recursivamente cnumerāvel L existem efetivamente um morfismo $\mathrm{h}_{0}$ e dois morfismos injetores $h_{1}$ e $h_{2}$ tais que $L=\left(e q\left(h_{1}, h_{2}\right)\right) h_{0}$ ?"

Uma resposta afirmativa para esta pergunta constituj um dos resultados principais desta dissertação e o objetivo deste capítulo è demonsträ-1o. Porẻm, faremos antes breves considerações sobre algumas tentativas que realizamos e as di ficuldades encontradas que, de certa forma, justificam a solu ção que adotamos na demonstração desse problema.

Inicialmente, utilizamos uma idéia semelhante ao acoplamento de uma máquina de Turing com a sua reversa, considerando para a gramātica $G=(N, \Sigma, S, P)$, que gera $L$, a gramática anälitica correspondente $G^{\prime}=\left(N, \Sigma, S, P^{\prime}\right)$ tal que

$L=L\left(G^{\prime}\right)=\left\{x \in \Sigma^{*} / x \Longrightarrow{ }^{*} S\right\}$. Porêm, os morfismos que obtivemos $\mathrm{G}^{\prime}$ ajnda não eram injetores.

Quando utilizamos gramáticas o ponto crucial é que podem existir ambas as produções $(\alpha, \beta)$ e $(\alpha, \gamma), \operatorname{com} \beta \neq \gamma$ ou $(\alpha, \beta)$ e $(\gamma, \beta)$, com $\alpha \neq \gamma$, fazendo com que os morfismos $h_{2}$ e $h_{1}$, respectivamente, não sejam injetores.

Assim, sem o emprego de gramäticas, obtivemos uma” demonstração que apresentamos a seguir.

Seja L uma 1inguagem recursivamente enumerävel e sc ja A uma mäquina de Turing deterministica que reconhece $L$, is 
to $\vec{e},|A|=L$. Então, pelo Tcorcma 1 (Capítulo 2) existe uma mäquina de Turing reversivel e normalizada $B$, equivalente a $A, \operatorname{com} I_{B}=\left\{z_{-}\right\}$.

Seja $C$ o acoplamento de $B$ com a sua reversa $B^{\prime}$.

Lembremos que C tambēm é reversível e normalizada, e observemos que $\Sigma_{A}=\Sigma_{B}=\Sigma_{C}=\Sigma$.

Podemos, então, associar à máquina de Turing $C$ dois cōdigos $f$ e g. Com isto, para cada palavra $s$ em $\Sigma^{*}$, existem dois cödigos $h_{1}$ e $h_{2}$ que são extensões de $f$ e $g$, respectivamente (como no Lema 1 do Capítulo 3). Mas, estamos interessados em morfismos $h_{0}, h_{1}$ e $h_{2}$ tais que qualquer que seja a palavra $s$ em $\Sigma^{*}$ (isto é, a entrada para a mäquina $C$ ),

$s \in L$ sse $s=w_{0}$, para algum $w$ em eq $\left(h_{1}, h_{2}\right)$.

ou seja, $h_{1}$ e $h_{2}$ não devem depender de nenhuma palavra s em $\Sigma^{*}$. Para isso devemos construir uma mäquina de Turing que seja ca paz de gerar cada uma das palavras em $\Sigma^{*}$, a partir da palavra vazia como entrada.

Consideremos então uma máquina de Turing reversível e semi-normalizada $D, \operatorname{com} I_{D}=\left\{q_{-}\right\}, F_{D}=\left\{q_{+}\right\}$e que enumera as palavras de $\Sigma^{*}$ de modo que, partindo com a configuração inicial de suas fitas igual a $(1,1)$, sempre que o seu controle finito se encontrar no estado $q_{+}$, a configuração corresponden te de suas fitas serā $(1, s)$, para alguma palavra s em $\Sigma^{*}$.Além disso, $D$ pode ser construida de modo a apresentar apenas uma aresta com origem em $q_{+}$e cujo rótulo seja $N \times N$. As palavras 
enumeradas por $D$ säo as reversas daquelas obtidas em ordem le xicográsica.

Denotando-se os elenientos do alfabeto $\Sigma$ por $\sigma_{1}, \sigma_{2}, \ldots, \sigma_{n}$, para algum $n \geq 1$, podemos representar graficamen te a mäquina de Turing $D$ e a sua reversa $D^{\prime}$ através da Figura 2 .
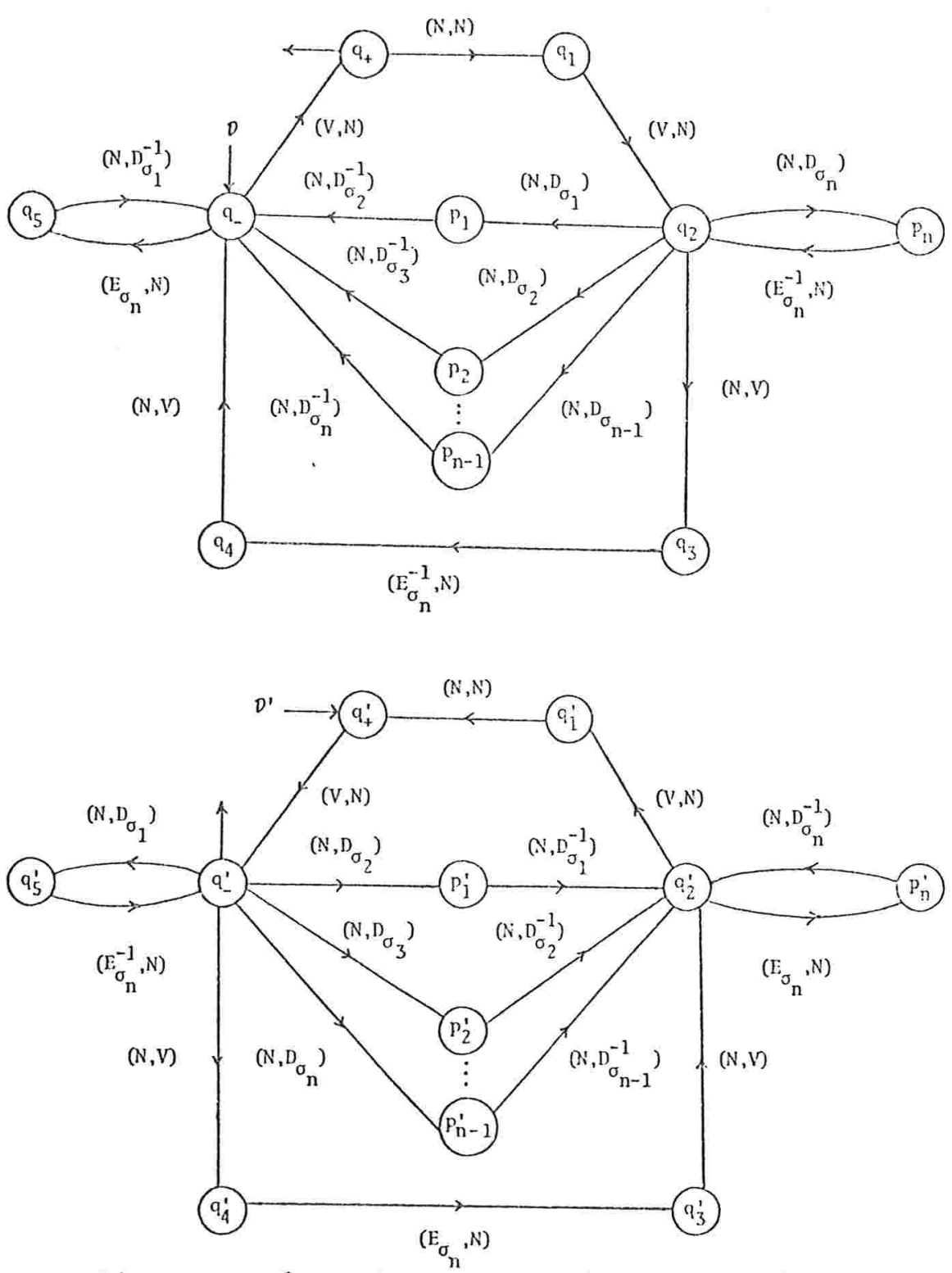

Figura 2 - A máquina de Turing reversível e semi-normalizada $D$ que enumera $\Sigma^{*} \mathrm{c} z$ sua reversa $D^{\prime}$ 
E imediato verificar que $D$ apresenta as propriedades descritas

Desse modo, uma computação cuja codificação tenha como imagem por $h_{0}$ uma palavra $\mathrm{s}$ em $\mathrm{L}$, consistirá em, inicial mente, processar a máquina $D$ com a configuração inicial de suas fitas igual a $(1,1)$ atē gerar alguma palavra $s$ em $\Sigma^{*}$, is to é, até que a configuração de suas fitas seja $(1, s)$. Então, executa-se a mãquina $C, \operatorname{com}(1, s)$ como a configuração inicial de suas fitas. C atingirá o seu estado final com a confi guração de suas fitas igual a inicial sses pertencer a L. Se isto ocorrer, executa-se a mâquina $D^{\prime}$ partindo-se com a confi guração de suas fitas igual a $(1, s)$. D' efetuará a computação reversa daquela executada por $D$, atingindo o seu estado final tendo $(1,1)$ como a configuração de suas fitas.

Assim, com esse objetivo, construimos uma mäquina de Turing $S$, "concatenando-se" as māquinas $D, C$ e $D$ ' através de três novas arestas. Então, a extensão dos morfismos associados a $S$ não mais dependerão de nenhuma palavra $s$ em $\Sigma^{*}$, já que a entrada para $S$ é a palavra vazia.

Descrevemos a seguir a māquina de Turing $S$ :

$$
\begin{aligned}
& \mathrm{T}_{S}=\mathrm{T}_{D} \cup \mathrm{T}_{C}, \\
& \Sigma_{S}=\Sigma, \\
& \mathrm{Q}_{S}=\mathrm{Q}_{D} \cup \mathrm{Q}_{C} \cup \mathrm{Q}_{D}, \mathrm{U}\{\lambda\},
\end{aligned}
$$

onde $Q_{D} \cap Q_{C}=\emptyset$ e $\lambda$ é um novo estado; 


$$
\begin{array}{ll} 
& I_{S}=I_{D}=\left\{q_{-}\right\}, \\
& F_{S}=F_{D^{\prime}}=\left\{q_{-}^{\prime}\right\} \\
\text { e } & E_{S}=E_{D} U E_{C} U E_{D^{\prime}}, U\{\eta, \mu, \nu\}, \\
\text { com } \quad \eta=\left(q_{+}, N, N, z_{-}\right), \text {onde }\left\{q_{+}\right\}=F_{D} \text { e }\left\{z_{-}\right\}=I_{C}, \\
\quad \mu=\left(z_{-}^{\prime}, N, N, \lambda\right), \text { onde }\left\{z_{-}^{\prime}\right\}=F_{C}, \\
\text { e } \quad v=\left(\lambda, N, N, q_{+}^{\prime}\right), \text { onde }\left\{q_{+}^{\prime}\right\}=I_{D} .
\end{array}
$$

Esquematicamente, podemos representar a máquina de Turing $S$ como segue (Figura 3).

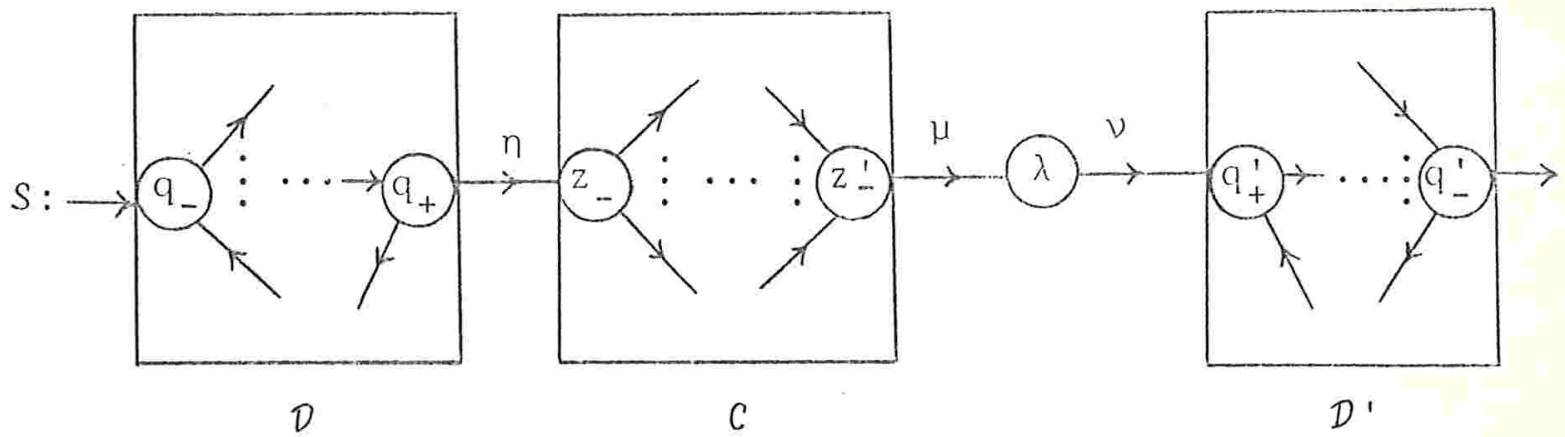

Figura 3 - Um esquema da mäquina de Turing $S$.

Podemos observar que $S$ é uma mäquina de Turing semi-normalizada, mas não é determinística e nem reversível. o não determinismo de $S$ resulta da existência em $A_{S}$ das arestas $\eta=\left(q_{+}, N, N, z_{-}\right)$e $\left(q_{+}, N, N, p\right)$, que é a ünica aresta de $D$.com or $\underline{i}$ gem em $q_{+}$, para algum $p$ em $Q_{D}$. $S$ não $\vec{e}$ reversível, pois existem em $A_{S}$ as arestas $\nu=\left(\lambda, N, N, q_{+}^{\prime}\right)$ e $\left(p^{\prime}, N, N, q_{+}^{\prime}\right)$. 
Agora, vamos associar a $S$ dois morfismos e cxaminar quais são as propriedades que eles apresentam.

Sejam os alfabetos

$$
\begin{aligned}
& \Gamma_{1}=\mathrm{T}_{S} U\{\varepsilon, \#\} \cup \mathrm{A}_{S} \cup \overline{\mathrm{T}}_{S} \cup\{\bar{\varepsilon}, \bar{H}\} \cup \overline{\mathrm{Q}}_{S} \\
& \Delta_{1}=\mathrm{T}_{S} \cup\{\varepsilon, \#\} \cup \mathrm{Q}_{S} \cup \overline{\mathrm{T}}_{S} \cup\{\bar{\varepsilon}, \overline{\bar{H}}\} \cup \overline{\mathrm{Q}}_{S},
\end{aligned}
$$

onde $\{\varepsilon, \#\} \cap \mathrm{T}_{S}=\emptyset$.

Consideremos os morfismos $f$ e $g: \Gamma_{1} \longrightarrow \Delta_{1}^{*}$, associa dos a $S$ e definidos como no Capítulo 3.

Devemos ressaltar que as restrições de $f$ e g a cada uma das mäquinas $D, C$ ou $D^{\prime}$ correspondem exatamente aos morfis mos associados a cada uma delas. Portanto, embora $f$ e g não sejam injetores, resulta pela Proposição 2 (Capitulo 3) que f e g restritos a $D$, a $C$ ou a $D$ ' são côdigos de atraso um.

Sejam os alfabetos $\Gamma_{2}=\Gamma_{1} U\{\vdash,-1\}$ e $\Delta_{2}=\Delta_{1} U\{\vdash,-1\}$ e os morfismos $f_{1}$ e $g_{1}: \Gamma_{2}^{*} \longrightarrow \Delta_{2}^{*}$, extensões dos morfismos $f$ e $g$, respectivamente. Definimos $f_{1}$ e $g_{1}$ pela Tabela 2 , na qual o denota um símbolo em $\mathrm{T}_{S}$, q e r são elementos de $Q_{S}$ e $(q, i, j, r)$ representa uma aresta em $A_{S}$.

Observemos tambẻm a importância fundamental da máquina de Turing $D$, fazendo com que os morfismos $f_{1}$ e $g_{1}$ independam de qualquer palavra em $\Sigma^{*}$; o que não ocorria com os morfismos $h_{1}$ e $h_{2}$ na demonstração do Lema 1 (Capítulo 3).

Como jả vimos, a máquina de Turing $S$ não é determi- 


\begin{tabular}{|c|c|c|}
\hline$\gamma \in \Gamma_{2}$ & $\gamma f_{1}$ & $r g_{1}$ \\
\hline$\sigma$ & $\bar{\sigma}$ & $\sigma$ \\
\hline $\bar{\sigma}$ & $\sigma$ & $\bar{\sigma}$ \\
\hline$\varepsilon$ & $\bar{\varepsilon}$ & $\varepsilon$ \\
\hline $\bar{\varepsilon}$ & $\varepsilon$ & $\bar{\varepsilon}$ \\
\hline H & $\bar{H}$ & H \\
\hline $\bar{H}$ & H & $\bar{H}$ \\
\hline$\left(q, E_{\sigma}, N, r\right)$ & $\overline{\mathrm{r}}$ & $\sigma \mathrm{q}$ \\
\hline$\left(q, N, D_{\sigma}, r\right)$ & $\bar{r}$ & $\mathrm{q}^{\sigma}$ \\
\hline$(q, V, N, r)$ & $\bar{\varepsilon} \bar{r}$ & $\varepsilon q$ \\
\hline$(q, N, V, r)$ & $\bar{r} \bar{\varepsilon}$ & $q \varepsilon$ \\
\hline$\left(q, E_{\sigma}^{-1}, N, r\right)$ & $\bar{\sigma} \bar{r}$ & $q$ \\
\hline$\left(q, N, D_{\sigma}^{-1}, r\right)$ & $\bar{r} \bar{\sigma}$ & $q$ \\
\hline$(q, N, N, r)$ & $\bar{r}$ & $q$ \\
\hline $\bar{q}$ & $q$ & $\bar{q}$ \\
\hline$\vdash$ & $\vdash \varepsilon q_{-} \varepsilon H$ & $\vdash$ \\
\hline-1 & -1 & $\bar{H} \varepsilon q_{-}^{\prime} \varepsilon^{-1}$ \\
\hline
\end{tabular}

Tabela 2 - Definição dos morfismos $f_{1}$ e $g_{1}$, extensões, respectivamente, dos morfismos.f e g associa dos a $S$. 
nística e nem reversível, e, de fato, os morfismos $f_{1}$ e $g_{1}$ não são injetores. Então, sem efetuar alterações em $S$, vamos construir, a partir dos morfismos $f_{1}$ e $g_{1}$, dois novos morfismos $h_{1}$ e $h_{2}$, respectivamente, que sejam injetores.

Sejam os alfabetos

$$
\Gamma=\Gamma_{2} U \vec{\Sigma} U \overline{\bar{\Sigma}} U\{\dot{\varepsilon}, \vec{H}, \overline{\bar{\varepsilon}}, \bar{\varepsilon}, \overline{\bar{\varepsilon}}, \overline{\bar{H}}\}
$$

e

$$
\Delta=\Delta_{2} U\{\phi, \phi\},
$$

onde $\oint$ e $\$$ são símbolos distintos entre si e não pertencem a $\triangle_{2}$

Sejam os morfismos $h_{1}$ e $h_{2}: \Gamma^{*} \longrightarrow \Delta^{*}$, definidos pela Tabela 3, onde o denota um símbolo em $\mathrm{T}_{S} ; \mathrm{q}, \mathrm{r}, \lambda_{,}, z_{-}, \mathrm{z}_{-}^{\prime}, \mathrm{q}_{+}$e $q_{+}^{\prime}$ são elementos de $Q_{S}$ e $(q, i, j, r)$, com $i \in E_{S}$ e $j \in D_{S}$ representa uma aresta em $\mathrm{A}_{S}$.

Precisamos mostrar então que os morfismos $h_{1}$ e $h_{2}$ são injetores, sendo que $h_{1} \vec{e}$ de atraso um da esquerda para a direita e $h_{2}$ da direita para a esquerda.

Além disso, devemos provar que $L=\left(\right.$ eq $\left.\left(h_{1}, h_{2}\right)\right) h_{0}$, onde $\mathrm{h}_{0}: \Gamma^{*} \longrightarrow \Sigma^{*} \overline{\mathrm{e}}$ um morfismo definido como segue: se $\gamma=\bar{\sigma}, \operatorname{com} \sigma$ em $\Sigma$, então $\gamma h_{0}=\sigma$; caso contrário, $\gamma h_{0}=1$. Obtemos assim o seguinte resultado.

TEOREMA 3 - Para cada 1inguagem recursivamente. enumeráve1 $\mathrm{L} \subseteq \Sigma^{*}$, existem efetivamente alfabetos $\Gamma$ e $\Delta$, um morfismo $\mathrm{h}_{0}: \Gamma^{*} \longrightarrow \Sigma^{*}$ e códigos $\mathrm{h}_{1}, \mathrm{~h}_{2}: \Gamma^{*} \longrightarrow \Delta^{*}, \operatorname{com}_{1}\left(\mathrm{~h}_{2}\right)$ de a- 


\begin{tabular}{|c|c|c|}
\hline rel' & $\mathrm{\gamma h}_{1}$ & $\gamma \mathrm{h}_{2}$ \\
\hline$\vdash$ & $1-\phi \phi \varepsilon \phi \$ q_{-} \phi \phi \varepsilon \phi \$ H \phi \phi$ & 1 \\
\hline$\dashv$ & -1 & 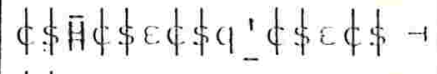 \\
\hline$\sigma$ & $\bar{\sigma} \phi \phi$ & $\phi \$ 0$ \\
\hline $\bar{\sigma}$ & $\sigma \phi \$$ & $\phi \$ \bar{\sigma}$ \\
\hline$\varepsilon$ & $\bar{\varepsilon} \phi \phi$ & $\phi \phi \varepsilon$ \\
\hline $\bar{\varepsilon}$ & $\varepsilon \notin \$$ & $\phi \$ \bar{\varepsilon}$ \\
\hline$H$ & $\overline{\mathrm{H}} \phi \phi$ & $\phi \$ H$ \\
\hline$\overline{\mathrm{H}}$ & $H \phi \phi$ & $\phi \$ \bar{H}$ \\
\hline$\left(q, E_{\sigma}, N, r\right)$ & $\bar{r} \phi \$$ & $\phi \$ \sigma \phi \$ q$ \\
\hline$\left(q, N, D_{\sigma}, r\right)$ & $\bar{r} \phi \$$ & $\phi \$ q \phi \$ \sigma$ \\
\hline$(q, V, N, r)$ & $\bar{\varepsilon} \phi \$ \bar{r} \phi \phi$ & $\$ \$ \varepsilon c \$ q$ \\
\hline$(q, N, v, r)$ & $\bar{r} \phi \$ \bar{\varepsilon} \phi \$$ & $\phi \$ q \phi \$ \varepsilon$ \\
\hline$\left(q, E_{\sigma}^{-1}, N, r\right)$ & $\bar{\sigma} \phi \$ \bar{r} \phi \$$ & $\phi \$ q$ \\
\hline$\left(q, N, D_{\sigma}^{-1}, r\right)$ & $\bar{r} \phi \$ \bar{\sigma} \phi \$$ & $\phi \$ q$ \\
\hline$(q, N, N, r) \in A_{S} \backslash\{n, \mu, \nu\}$ & $\bar{r} \phi \$$ & $\$ \$ q$ \\
\hline$\eta=\left(q_{+}, N, N, z_{-}\right)$ & $\bar{z} \&$ & $\phi \$ q_{+} \phi$ \\
\hline$\mu=(z !, N, N, \lambda)$ & $\bar{\lambda} \phi \$$ & $\$ \$ z !$ \\
\hline$\nu=\left(\lambda, N, N, q_{+}^{1}\right)$ & $\phi \$ \bar{q}_{+}^{\prime} \phi \$$ & $\lambda$ \\
\hline $\bar{q}, q \in Q_{S} \backslash\left\{z_{-}, \lambda\right\}$ & $q \notin \$$ & $\phi \$ \bar{q}$ \\
\hline $\bar{z}$ & $\$ z \_\$$ & $\$ \bar{z}=1$ \\
\hline & $\lambda$ & $\phi \$ \bar{\lambda} \phi \$$ \\
\hline $\bar{\sigma}, \quad \sigma \in \Sigma$ & $\$ \bar{\sigma} \phi$ & $\$ \sigma \phi$ \\
\hline & $\$ \bar{\varepsilon} \phi$ & $\$ \varepsilon \notin$ \\
\hline$\underline{B}$ & $\$ \bar{H} \phi$ & $\$ H \phi$ \\
\hline$\overline{\bar{\varepsilon}}$ & $\$ \varepsilon \phi$ & $\phi \bar{\varepsilon} \phi$ \\
\hline $\bar{\varepsilon}$ & $\$ \$ \bar{\varepsilon}$ & $\varepsilon \phi \$$ \\
\hline $\bar{\sigma}, \quad \sigma \in \Sigma$ & $\phi \$ \sigma$ & $\bar{\sigma} \phi \$$ \\
\hline $\bar{\varepsilon}$ & $\phi \$ \varepsilon$ & $\bar{\varepsilon} \phi \$$ \\
\hline $\bar{H}$ & $\notin \$ H$ & $\bar{H} \phi \$$ \\
\hline
\end{tabular}

Tabela 3 - Definição dos morfismos $h_{1}$ e $h_{2}$, obtidos a par tir de $f_{1}$ e $g_{1}$, respectivamente. 
traso um da esquerda para a direita (da dircita para a esquer da) tais que $L=\left(\mathrm{cq}_{1}\left(\mathrm{~h}_{1}, \mathrm{~h}_{2}\right)\right) \mathrm{h}_{0}$.

A demonstração do Teorema 3 fica completa com os seis lemas apresentados a seguir.

$\underline{\text { LEMA } 1}$ - 0 morfismo $h_{2}$ é injetor sobre $\Gamma$.

LEMA 2 - 0 morfismo $h_{2}$ é um código de atraso um da direita para a esquerda.

$\underline{\text { LEMA } 3}$ - O morfismo $\mathrm{h}_{1} \overline{\mathrm{e}}$ injetor sobre $\Gamma$.

LEMA 4 - O morfismo $h_{1}$ è um cỏdigo de atraso um da esquerda para a direita.

$\underline{\text { LEMA } 5}-\mathrm{L} \subseteq\left(\mathrm{eq}\left(\mathrm{h}_{1}, \mathrm{~h}_{2}\right)\right) \mathrm{h}_{0}$.

$\underline{\text { LEMA } 6}-\left(\mathrm{eq}\left(\mathrm{h}_{1}, \mathrm{~h}_{2}\right)\right) \mathrm{h}_{0} \subseteq \mathrm{L}$.

監

Vamos demonstrar então cada um dos 1 emas.

Para isso definimos inicialmente dois morfismos

$\mathrm{h}_{\mathrm{e}}: \Delta_{1}^{*} \longrightarrow\left(\phi \$ \Delta_{1}\right)^{*}$ e $\mathrm{h}_{\mathrm{d}}: \Delta_{1}^{*} \longrightarrow\left(\Delta_{1} \phi \$\right)^{*}$, tais que, para ca. da $\delta$ em $\Delta_{1}$

$$
\delta h_{\mathrm{e}}=\phi \$ \delta \quad \text { e } \delta \mathrm{h}_{\mathrm{d}}=\delta \phi \$ \text {. }
$$

Desse modo, se $\delta \in \Delta_{1}^{*}, \operatorname{com} \delta=\delta_{1} \delta_{2} \ldots \delta_{n}, \delta_{i}$ em $\Delta_{1}(1 \leq i \leq n)$ 
cntão,

$$
\delta h_{e}=\phi \$ \delta_{1} \phi \$ \delta_{2} \ldots \phi \$ \delta_{n} \quad \text { e } \delta h_{d}=\delta_{1} \phi \$ \delta, \phi \$ \ldots \delta_{n} \phi \$ .
$$

Demonstração do Lema 1

Queremos mostrar que $h_{2} \vec{e}$ injetor sobre $\Gamma$.

Sejam $x$ e y em $\Gamma$ tais que $x h_{2}=y h_{2}$.

- Se $x h_{2}$ pertence a $\left\{r,\left(\bar{H} \varepsilon q_{-}^{\prime} \varepsilon-1\right) h_{e}\right\} U\left(T_{S} U^{-}{ }^{-} U\{\varepsilon, \bar{\varepsilon}, H, \bar{H}\} \quad U\right.$

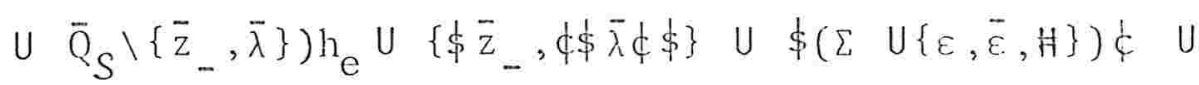

$$
\begin{aligned}
& \mathrm{U}(\bar{\Sigma} \cup\{\varepsilon, \bar{\varepsilon}, \bar{H}\}) \mathrm{h}_{\mathrm{d}} \mathrm{U}\left\{\not \$ \mathrm{q}_{+} \phi, \lambda\right\}
\end{aligned}
$$

segue pela definição de $h_{2}$ que $x=y$.

- Se $x h_{2}=\$ \$ z_{-}^{\prime}$, então $x=\mu=\left(z_{-}^{\prime}, N, N, \lambda\right)$ que é a ünica aresta de $S$ com origem em $z_{-}^{\prime}$. Logo, pela definição de $h_{2}$ segue que $x=y$.

- Se $x h_{2}$ pertence a $\left(Q_{S} \backslash\left\{z_{-}^{\prime}\right\} U\left(T_{S} \cup\{\varepsilon\}\right) Q_{S} \cup Q_{S}\left(T_{S} U\{\varepsilon\}\right)\right) h_{e}$, então de $x h_{2}=y h_{2}$ segue que $x g h_{e}=y g h_{e} ; 10 g o, x g=y g$. Lembremos que g é um dos morfismos associados a $S$.

Mas, por construção de $S$ e pela definição de $h_{2}$, $x$ é uma aresta que pertence a exatamente uma das máquinas $D$, C ou $D^{\prime}$ e y também è uma aresta que pertence à mesma máquina a qual $x$ pertence. 
Por outro lado, a restrição de g a cada uma das máquinas $D, C$ ou $D^{\prime} \vec{e}$ exatamente o correpondente morfismo associado a cada uma delas e que pela proposição $2 a$ (Capítú 103 ) ê injetor sobre o seu respectivo domínio, jả que $D$, $C$ e $D^{\prime}$ são determinísticas.

Portanto, concluímos que $\mathrm{x}=\mathrm{y}$.

Assim, $h_{2} \vec{e}$ um morfismo injetor sobre $\Gamma$.

Demonstração do Lema 2

Queremos mostrar que o morfismo $\mathrm{h}_{2} \overline{\mathrm{e}}$ um côdigo de $\underline{\text { a }}$ traso um da direita para a esquerda.

Como pelo Lema 1 o morfismo $h_{2} \bar{e}$ injetor sobre $\Gamma$, é suficiente demonstramos que $h_{2}$ é de atraso um da direita pa ra a. esquerda.

Sejam u e v em $\Gamma h_{2}$ e w em $\Delta^{*}$ tais que wvu $\epsilon\left(\Gamma h_{2}\right)$ * Então, existe $m \geq 1$ e $z_{1}, z_{2}, \ldots, z_{m}$ em $\Gamma h_{2}$ tais que

$$
\text { wvu }=z_{m} z_{m-1} \cdots z_{1} \text {. }
$$

Precisamos mostrar que wV tambëm pertence a $\left(\Gamma h_{2}\right) *$. Com esta finalidade, vamos examinar cada uma das possibilidades para $u$ em $\Gamma h_{2}$.

- Se u pertence a $\left\{1,\left(\bar{H} \varepsilon q_{-}^{\prime} \varepsilon-1\right) h_{e}\right\} \cup\left(\bar{T}_{S} \cup\left\{\bar{\varepsilon}, \#, \bar{F}_{,}\right\} \cup \overline{\mathrm{Q}}_{S} \backslash\left\{\bar{z}_{-}, \bar{\lambda}\right\}\right) h_{e} U$ 


$$
\begin{aligned}
& U\left\{\phi \$ q_{+} \phi\right\} \cup\left\{\$ \bar{z}_{-}, \phi \phi \bar{\lambda} \phi \phi\right\} \cup \quad \$\left(\sum \cup\{\varepsilon, \bar{\varepsilon}, H\}\right) \phi U \\
& U(\bar{\Sigma} \cup\{\varepsilon, \bar{\varepsilon}, \bar{H}\}) h_{(l},
\end{aligned}
$$

então, pela definição de $h_{2}$, não existe uma palavra $t$ cm $\Delta^{+}$tal que $z_{1}=$ tu ou que $u=t z_{1}$. Logo, $z_{1}=u$. Portanto, de (1) temos que $w V=z_{m} z_{m-1} \cdots z_{2} \in\left(\Gamma h_{2}\right) *$.

- Se u=\$\$z:- então, pela definição de $h_{2}$ e pelo fato de $\mu=\left(z_{-}^{\prime}, N, N, \lambda\right)$ ser a ünica aresta de $S$ com origem cm $z_{-}^{\prime}$, segue que $z_{1}=u$. Logo, de (1) temos que wv $\in\left(\Gamma h_{2}\right) *$.

- Se $u=\lambda$ resulta pela definição de $h_{2}$ e pelo fato de $\nu=\left(\lambda, N, N, q_{+}^{\prime}\right)$ ser a única aresta de $S$ com origem em $\lambda$ que $z_{1}=u$. Logo, de (1) $w v=z_{m} z_{m-1} \cdots z_{2} \in\left(\Gamma h_{2}\right) *$.

- Se $u=\$ \$ \sigma, \sigma$ em $T_{S}$ e $z_{1} \neq u$, segue pela definição de $h_{2}$ e de (1) que $z_{1}=\$ \$ q \phi \$ \sigma$, para algum $q$ em $Q_{S}\left(q \neq \lambda\right.$, pois $v=\left(\lambda, N, N, q_{+}^{\prime}\right)$ é a ünica aresta de $S$ com origem em $\lambda)$, e a aresta $\alpha=\left(q, N, D_{\sigma}, r\right)$ ta1 que $\alpha h_{2}=z_{1}$, pertence a exatamente uma das máquinas $D$, C ou $D^{\prime}$. Então, de (1) resulta que ocorre um dos seguintes casos:

(i) $\quad v=\phi \$$.

o que implica que uma das arestas $\beta=\left(q, E_{\delta}^{-1}, N, r\right)$ ou $\beta=\left(q, N, D_{\delta}^{-1}, r\right)$ ou $B=(q, N, N, r)$ pertence a $A_{S}$, com $B h_{2}=v \quad e$ $\beta \notin\{\eta, \mu, \nu\}$. 
(ii) $v=\$ \$ \varepsilon \phi \$$.

Nesse caso, a aresta $\beta=(q, V, N, r)$ pertence a $\Lambda_{S}$ e $B h_{2}=v$.

(iii) $v=\$ \$ \tau \phi \$ q$, para algum $\tau$ em $\mathrm{T}_{S}$.

Então, a aresta $\beta=\left(q, E_{\tau}, N, r\right)$ pertence a $A_{S}$ e $B h_{2}=v$. Mas, em qualquer um desses casos, $\beta$ deve pertencer à mesma mäquina a qual a pertence. Isto contradiz o fato de que $D, C$ ou $D^{\prime}$ é determinística. Portanto, $z_{1}=u$ e de (1) segue que wr $\in\left(\Gamma h_{2}\right) *$.

- Se $u=\$ \$ \varepsilon$, a demonstração ê anâloga a anterior.

- Se $u=\$ \$ q$, com $q$ em $Q_{S} \backslash\left\{z_{-}^{\prime}\right\}$, então uma das arestas $\alpha=\left(q, E_{\sigma}^{-1}, N, r\right)$ ou $\alpha=\left(q, N, D_{\sigma}^{-1}, r\right)$ ou $\alpha=(q, N, N, r)$, tal que $\alpha \notin\{n, \mu, \nu\}$ e $\alpha h_{2}=u$, pertence a exatamente uma das máquinas $D, C$ ou $D^{\prime}$.

Suponhamos que $z_{1} \neq u$. Então, pela definição de $h_{2}$ e de (1) segue que $z_{1}=\phi \$ \varepsilon \phi \$ q$ ou $z_{1}=\phi \$ \tau \phi \$ q$, para algum $\tau$ em $\mathrm{T}_{S}$. Logo, resulta, respectivamente, que a aresta $\beta=(q, V, N, t)$ ou $\beta=\left(q, E_{\tau}, N, t\right)$ pertence à mesma máquina a qual $\alpha$ pertence e $B h_{2}=z_{1}$. Mas, isto é um absurdo, pois $D, C$ e $D^{\prime}$ são deter ministicas. Portanto, $z_{1}=u$ e wV $\in\left(\Gamma h_{2}\right)^{*}$.

- Seja $u=\$ \$ q \phi \$ \sigma$, com q em $Q_{S}$ e $\sigma$ em $T_{S}$. (Observemos que q $\neq \lambda$.) 
Então, a aresta $\alpha=\left(q, N, I_{\sigma}, r\right)$ pertence a cxatamente uma das máquinas $D, C$ ou $D^{\prime}$ e $\alpha h_{2}=u$.

Se $z_{1} \neq u$, segue pela definição de $h_{2}$ e de (1) que $z_{1}=\$ \$ \sigma$ e ocorre para $z_{2}$ uma das possibilidades abaixo:

(i) $\quad z_{2}=\$ \$$.

Então, uma das arestas $\beta=\left(q, E_{\gamma}^{-1}, N, t\right)$ ou $\beta=\left(q, N, D_{\gamma}^{-1}, t\right)$ ou $\beta=(q, N, N, t)$ pertence a $A_{S}, \operatorname{com} B h_{2}=z_{2}$ e $\beta \notin\{\eta, \mu, v\}$.

(ii) $z_{2}=\phi \$ \varepsilon \phi \$ q$.

Nesse caso, a aresta $B=(q, V, N, t)$ pertence a $A_{S}$ e $\mathrm{Bh}_{2}=\mathrm{z}_{2} \cdot$

(iii) $z_{2}=\phi \$ \tau \phi q$, para algum $\tau$ em $\mathrm{T}_{S}$.

Então, a aresta $\beta=\left(q, E_{\tau}, N, t\right)$ pertence a $A_{S}$ e $B h_{2}=z_{2} \cdot$

Porëm, em qualquer un dos casos acima, $\beta$ deve pertencer à mesma máquina a qual a pertence. o que contradiz - fato de $D, C$ ou $D^{\prime}$ ser determinística. Portanto, $z_{1}=u$ e de (1) wv $\in\left(\Gamma h_{2}\right) *$.

- Se $u=\not \$ q \not \$ \varepsilon$, com q em $Q_{S}$, a demonstração é análoga a anterior. 
- Seja $u=\downarrow \$ \sigma \phi \$ q$, com a cm Q $Q_{S}$ o $\mathrm{cm} \mathrm{T}_{S}$. (Observemos que q $\neq \lambda$. ) Então, a aresta $\alpha=\left(\mathrm{q}, \mathrm{E}_{\sigma}, \mathrm{N}, \mathrm{r}\right)$ pertence a exatamente uma das mäquinas $D, C$ ou $D^{\prime}$ e $\alpha h_{2}=u$.

Se $z_{1} \neq u$, resulta pela definição de $h_{2}$ e de (1) que $z_{1}=\$ \$ q$. Consequentemente, uma das arestas $B=\left(q, E_{\tau}^{-1}, N, t\right)$ ou $\beta=\left(q, N, D_{\tau}^{-1}, t\right)$ ou $\beta=(q, N, N, t)$ pertence à mesma māquina a qual a pertence e $B h_{2}=z_{1}$. Porém, é impossível que isto aconteça, jä que $D, C$ e $D^{\prime}$ são determinísticas. Logo, $z_{1}=u$ e de (1) segue que wV $\in\left(\Gamma h_{2}\right) *$.

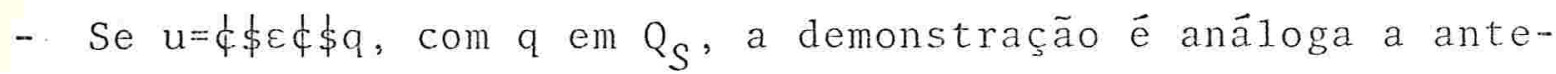
rior.

Assim, concluímos que o morfismo $h_{2}$ é un côdigo de atraso um da direita para a esquerda.

圈

Observação - 0 morfismo $h_{2}$ não è de atraso 1imitado da esquerda para a direita.

Suponhamos, por absurdo, que exista um inteiro $n \geq 0$ ta1 que $h_{2}$ seja de atraso $n$ da esquerda para a direita. Ou se ja, para quaisquer palavras u em $\Gamma h_{2}, v$ em $\left(\Gamma h_{2}\right)^{n}$, com $v=v_{1} v_{2} \cdots v_{n}, v_{i}$ em $\Gamma h_{2}(1 \leq i \leq n)$ e w em $\Delta^{*}$, se uvw $\in\left(\Gamma h_{2}\right) *$ então vw $\in\left(\Gamma h_{2}\right) *$.

Sejam $u=\$ \$ q_{+}$e para cada $i(1 \leq i \leq n), \quad v_{i}=\phi \$ \sigma_{i}, \quad$ com $\sigma_{i}$ em $T_{S}$. Então, $v=v_{1} v_{2} \ldots v_{n} \epsilon\left(\Gamma h_{2}\right)^{n}$. Seja $w=\phi$. Logo, 


$$
u v w=\phi \$ q_{+} \phi \$ \sigma_{1} \phi \$ \sigma_{2} \ldots \phi \$ \sigma_{n} \phi \in\left(\Gamma h_{2}\right)^{*} .
$$

Basta observarmos que $u v w=w_{0} w_{1} \cdots w_{n}$, onde

$w_{0}=\$ \$ q_{+} \phi \in \Gamma h_{2}$ e para cada cada i $(1 \leq i \leq n), w_{i}=\$ \sigma_{i} \phi \in \Gamma h_{2}$. Mas, $v w=\phi \$ \sigma_{1} \phi \$ \sigma_{2} \ldots \phi \phi \sigma_{n} \phi \notin\left(\Gamma h_{2}\right)^{*}$.

Portanto, concluímos de (2) que $\mathrm{h}_{2}$ não é de atraso limitado da esquerda para a direita.

\section{Demonstração do Lema 3}

$$
\text { Queremos mostrar que o morfismo } h_{1} \text { é injetor sobre }
$$

$\Gamma$.

$$
\text { Sejam } x \text { e } y \text { em } \Gamma \text { tais que } x h_{1}=y h_{1} \text {. }
$$

- Se $x h_{1}$ pertence a $\left\{\left(1-\varepsilon q_{-} \varepsilon H\right) h_{d},-1\right\} \cup\left(T_{S} \cup \bar{T}_{S} \cup\{\varepsilon, \bar{\varepsilon}, H, \bar{H}\} \quad U\right.$

$$
\begin{aligned}
& \left.U Q_{S} \backslash\left\{z_{-}, \lambda\right\}\right) h_{d} U\left\{\$ \phi_{-} \phi \$, \lambda\right\} \cup \$(\bar{\Sigma} \cup\{\varepsilon, \bar{\varepsilon}, \bar{H}\}) \phi U \\
& U(\Sigma \cup\{\varepsilon, \bar{\varepsilon}, H\}) h_{\mathrm{e}} U\left\{\bar{z}_{-} \phi, \phi \$ \bar{q}_{+}^{\prime} \phi \$\right\}
\end{aligned}
$$

segue pela definição de $h_{1}$ que $x=y$.

- Se $x h_{1}=\bar{\lambda} \phi \$$, então $x=\mu=\left(z_{-}^{\prime}, N, N, \lambda\right)$ que é a unica aresta de $S$ com término em $\lambda$. Logo, pela definição de $h_{1}$ resul ta que $x=y$.

- Se xh ${ }_{1}$ pertence a $\left(\bar{Q}_{S} \backslash\{\bar{\lambda}\} \cup\left(\bar{T}_{S} \cup\{\bar{\varepsilon}\}\right) \bar{Q}_{S} \cup \bar{Q}_{S}\left(\bar{T}_{S} \cup\{\bar{\varepsilon}\}\right)\right) h_{d}$, 
então de $x h_{1}=y h_{1}$ segue que $x f h_{d}=y f h_{d} ; 10 g o, x f=y f$.

Mas, por construção de $S$ e pela definição de $h_{1}, x$ $\vec{e}$ uma aresta pertencente a exatamente uma das máquinas $D$, $C$ ou $D^{\prime}$ e y também é uma aresta que pertence à mesma máquj. na a qual $x$ pertence.

Por outro lado, a restrição de $f$ a cada uma das má quinas $D, C$ ou $D^{\prime}$ é exatamente o correspondente morfismo as sociado a cada uma delas e que pela preposição 2c (Capítu103 ) è injetor sobre o seu respectivo domínio, já que $D$, $C$ e $D^{\prime}$ são reversíveis.

$$
\begin{aligned}
& \text { Portanto, resulta que } x=y \text {. } \\
& \text { Assim, o morfismo } h_{1} \bar{e} \text { injetor sobre } \Gamma \text {. }
\end{aligned}
$$

\section{Demonstração do Lema 4}

Queremos mostrar que o morfismo $h_{1}$ é um código de atraso um da esquerda para a direita.

Como pelo Lema 3 o morfismo $h_{1}$ é injetor sobre $\Gamma$, é suficiente demonstrarmos que $h_{1}$ é de atraso um da esquerda para a direita.

Sejam u e vem $\Gamma h_{1}$ è w em $\Delta^{*}$ tais que uvw $\in\left(\Gamma h_{1}\right)^{*}$. Então, existe $m \geq 1$ e $z_{1}, z_{2}, \cdots, z_{m}$ em $\Gamma h_{1}$ tais que

$$
u v w=z_{1} z_{2} \cdots z_{m} \cdot
$$

Precisamos mostrar que vw também pertence a $\left(\Gamma h_{1}\right)$ *. 
Com esta finalidade, vamos analisar cada uma das possibiljaldes para $u \mathrm{em} \mathrm{Th}_{1}$.

- Se u pertence a $\left\{\left(1-\varepsilon q_{-} \varepsilon \beta^{\prime}\right) h_{d},-1\right\} \cup\left(\mathrm{T}_{S} U\{\varepsilon, H, \vec{\not}\} \cup Q_{S} \backslash\left\{z_{-}, \lambda\right\}\right) h_{d} U$ $U\{\phi \$ \bar{q}+\phi \$\} \cup\left\{\$ \neq \_\$, \lambda\right\} \cup \$(\bar{\Sigma} \cup\{\varepsilon, \bar{\varepsilon}, \bar{H}\}) \phi U(\Sigma \cup\{\varepsilon, \bar{\varepsilon}, H\}) h_{\mathrm{e}}$,

então, pela definição de $h_{1}$ não existe uma palavra $t$ em $\Delta^{+}$ tal que $z_{1}=u t$ ou que $u=z_{1} t \cdot \operatorname{Logo}, z_{1}=u$.

Portanto, de (3) temos que vw $=z_{2} \cdots z_{m} \in\left(\Gamma h_{1}\right) *$.

- Se u $=\bar{\lambda} \phi \$$ então, pela definição de $\mathrm{h}_{1}$ e mais o fato de $\mu=\left(z_{-}^{\prime}, N, N, \lambda\right)$ ser a ünica aresta de $S$ com término em $\lambda$, se gue que $z_{1}=u$. $\log \odot$, de (3) temos que vw $\in\left(\Gamma h_{1}\right) *$.

- Se $u=\bar{z}_{-} \phi$ resulta pela definição de $h_{1}$ e pelo fato de $\eta=\left(q_{+}, N, N, z_{-}\right)$ser a ünica aresta de $S$ com tërmino em $z_{-}$ que $z_{1}=u$. Logo, $\operatorname{de}(3)$ vw $=z_{2} \cdots z_{m} \in\left(\Gamma h_{1}\right) *$.

- Se $u=\bar{\sigma} \phi \$$, com o em $\mathrm{T}_{S}$ e $z_{1} \neq u$, segue pela definição de $h_{1}$ e de (3) que $z_{1}=\bar{\sigma} \phi \$ \bar{r} \phi \$$, para algum $r$ em $Q_{S}\left(r \neq z_{-}\right.$, pois $\eta=\left(q_{+}, N, N, z_{-}\right)$é a única aresta de $S$ com têrmino em $\left.z_{-}\right)$e a aresta $\alpha=\left(q, E_{\sigma}^{-1}, N, r\right)$ pertence a exatamente uma das mäquinas $D, C$ ou $D^{\prime}, \operatorname{com} \alpha h_{1}=z_{1}$. Então, de (3) segue que ocorre um dos seguintes casos: 
(i) $\quad v=\bar{r} \phi \$$.

Então, uma das arestas $\beta=\left(t, E_{\delta}, N, r\right)$ ou $\beta=\left(t, N, D_{\delta}, r\right)$ ou $B=(t, N, N, r)$ pertence a $A_{S}$ tal que $B h_{1}=v$ e $\beta \notin\{\eta, \mu, \nu\}$.

(ii) $\quad v=\bar{r} \notin \$ \bar{\varepsilon} \phi \$$.

Nesse caso, a aresta $\beta=(t, N, V, r)$ pertence a $A_{S} e$ $\beta h_{1}=v$

(iii) $v=\bar{r} \phi \$ \bar{\tau} \phi \$$, para algum $\tau$ em $\mathrm{T}_{S}$. Então, a aresta $\beta=\left(t, N, D_{\tau}^{-1}, r\right)$ pertence a $A_{S}$ e $\beta \mathrm{h}_{1}=\mathrm{v}$

Mas, em qualquer um desses casos, $\beta$ deve pertencer à mesma mảquina a qual a pertence. Isto contradiz o fato de que $D, C$ ou $D^{\prime}$ é reversível. Portanto, $z_{1}=u$ e de (3) se gue que vw $\in\left(\Gamma h_{1}\right)$ *.

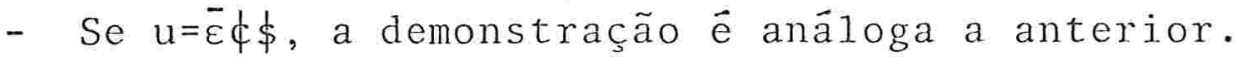

- Se $u=\bar{r} \phi \$$, com $r$ em $Q_{S} \backslash\{\lambda\}$, então uma das arestas $\alpha=\left(q, E_{\sigma}, N, r\right)$ ou $\alpha=\left(q, N, D_{\sigma}, r\right)$ ou $\alpha=(q, N, N, r)$ pertence a exatamente uma das māquinas $D, C$ ou $D^{\prime}, \operatorname{com}_{1} \alpha h_{1}=u$ e $\alpha \notin\{\eta, \mu, \nu\}$.

Suponhamos que $z_{1} \neq u$. Então, pela definição de $h_{1}$ e de (3) segue que $z_{1}=\bar{r} \phi \$ \bar{\tau} \phi \$$, para algum $\tau$ em $\mathrm{T}_{S}$ ou $z_{1}=\bar{r} \phi \$ \bar{\varepsilon} \phi \$$. Logo, resulta, respectivamente, que a aresta $\beta=\left(t, N, D_{\tau}^{-1}, r\right)$ ou $\beta=(t, N, V, r)$ pertence à mesma mäquína a 
qual a pertence e $B h_{1}=z_{1}$. Mas, isto $\check{e}$ um absurdo, pois $D$, C e $D^{\prime}$ são reversíveis. Portanto, $z_{1}=u$ e de (3) vite( $\left.\Gamma h_{1}\right) *$.

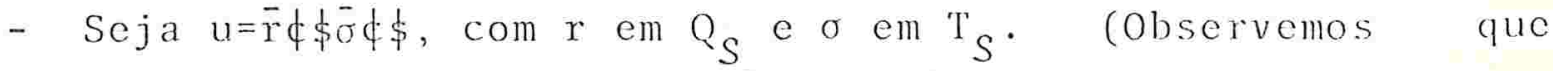
$\left.\mathrm{r} \neq \mathrm{z}_{-}.\right)$Então, a aresta $\alpha=\left(\mathrm{q}, \mathrm{N}, \mathrm{D}_{\mathrm{O}}^{-1}, \mathrm{r}\right)$ pertence a exatamente uma das mäquinas $D, C$ ou $D^{\prime}$ e $\alpha h_{1}=u$.

Se $z_{1} \neq u$, segue pela definição de $h_{1}$ e de ( 3 ) que $z_{1}=\bar{r} \not \phi \quad e$, neste caso, uma das arestas $\beta=\left(t, E_{\tau}, N, r\right)$ ou $\beta=\left(t, N, D_{\tau}, r\right)$ ou $B=(t, N, N, r)$ pertence à mesma mäquina a qual $\alpha$ pertence e $\beta h_{1}=z_{1}$. Porém, è impossível que isto ocorra, já que $D, C$ e $D^{\prime}$ são reversíveis. Logo, $z_{1}=u$ e de (3) resulta que vw $\in\left(\Gamma h_{1}\right) *$

- Se u= $\bar{r} \not \phi \bar{\varepsilon} \not \downarrow$, com r em $Q_{S}$, a demonstração é análoga a anterior.

- Se $u=\bar{\sigma} \phi \$ \bar{r} \phi \$$, com $\sigma$ em $\mathrm{T}_{S}$ e $r$ em $Q_{S}\left(r \neq z_{-}\right)$, a aresta $\alpha=\left(q, E_{\sigma}^{-1}, N, r\right)$ pertence a exatamente uma das mäquinas $D, C$ ou $D^{\prime}$ e $\alpha h_{1}=u$.

Suponhamos que $z_{1} \neq u$. Pela definição de $h_{1}$ e de (3) segue que $z_{1}=\bar{\sigma} \phi \$$ e ocorre uma das possibilidades abaixo pa ra $z_{2}$ :

(i) $\quad z_{2}=\bar{r} \phi \$$.

o que implica que uma das arestas $\beta=\left(t, E_{\delta}, N, r\right)$ ou 
$\beta=\left(t, N, D_{\delta}, r\right)$ ou $\beta=(t, N, N, r)$ pertence a $\Lambda_{S}$, com $\beta h_{1}=z_{2} \quad c$ $\beta \notin\{n, \mu, \nu\}$.

(ii) $\mathrm{z}_{2}=\overline{\mathrm{r}} \phi \$ \bar{\tau} \phi \$$, para algum $\mathrm{T}$ em $\mathrm{T}_{S}$.

Então, a aresta $B=\left(t, N, D_{T}^{-1}, r\right)$ pertence a $\Lambda_{S}$ e $B h_{1}=z_{2}$.

(iii) $z_{2}=\bar{r} \phi \phi \bar{\varepsilon} \phi \phi$.

Nesse caso, a aresta $\beta=(t, N, N, r)$ pertence a $A_{S} e$ $\beta h_{1}=z_{2} \cdot$

Porêm, em qualquer um desses casos, $B$ deve pertencer à mesma máquina a qual $\alpha$ pertence. Isto contradiz o fạ to de que $D, C$ ou $D^{\prime}$ é reversível. Portanto, $z_{1}=u$ e de (j) segue que vw $\in\left(\Gamma h_{1}\right) *$

- Se u= $\bar{\varepsilon} \phi \$ \bar{r} \phi \$$, com $r$ em $Q_{S}$, a demonstração é análoga a anterior.

Assim, concluímos que o morfismo $h_{1}$ é um côdigo de atraso um da esquerda para a direita.

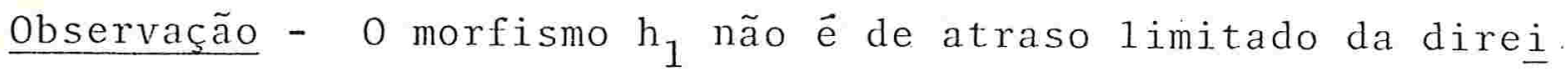
ta para a esquerda. 
Suponhamos, por absurdo, que exista um inteiro $n \geq 0$ tal que $h_{1}$ seja de atraso $n$ da direita para a esquerda. ou seja, para quaisquer palavras $u$ em $\Gamma h_{1}, v$ em $\left(\Gamma h_{1}\right)^{n}$, com $v=v_{n} v_{n-1} \cdots v_{1}, v_{i}$ em $\Gamma h_{1}(1 \leq i \leq n)$ e w em $\Delta *$, se wvu $\in\left(\Gamma h_{1}\right)$ * então wv $\in\left(\Gamma h_{1}\right)$ *.

$$
\text { Sejam } u=\bar{q}_{+}^{\prime} \phi \$ \text { e para cada } i(1 \leq i \leq n), v_{i}=\sigma_{i} \phi \$,
$$

com $\sigma_{i}$ em $T_{S}$. Então, $v=v_{n} v_{n-1} \cdots v_{1} \in\left(\Gamma h_{1}\right)^{n}$. Seja $w=\phi \$$. Logo,

$$
\text { wvu }=\phi \$ \sigma_{n} \phi \$ \sigma_{n-1} \phi \$ \ldots \sigma_{2} \phi \$ \sigma_{1} \phi \$ \bar{q}_{+}^{\prime} \phi \$ \in\left(\Gamma h_{1}\right)^{*} \text {. }
$$

Basta considerarmos $w_{v u}=w_{n} w_{n-1} \cdots w_{1} w_{0}$, onde $w_{0}=\phi \$ \bar{q}_{+}^{\prime} \phi \$ \in \Gamma h_{1}$ e para cada $i \quad(1 \leq i \leq n), w_{i}=\phi \$ \sigma \sigma_{i} \in \Gamma h_{1}$. Mas, wV $=\phi \$ \sigma_{n} \phi \$ \sigma_{n-1} \phi \$ \ldots \sigma_{2} \phi \$ \sigma_{1} \phi \$ \notin\left(\Gamma h_{1}\right) *$.

Portanto, resulta de (4) que $h_{1}$ não é de atraso limitado da direita para a esquerda.

Demonstração do Lema 5

Seja s uma palavra em L.

Queremos provar que existe em eq $\left(h_{1}, h_{2}\right)$ uma palavra w tal que $w_{0}=s$. Essa palavra w será uma codificação da com putação da máquina $S$ para a palavra $s$. 
Como $s \in \Sigma^{*}$ existe em $D$ um passeio $c: q_{-} \longrightarrow q_{+}$ta1

que

$$
\left(\varepsilon q_{-} \varepsilon\right)|| c||=\varepsilon q_{+} s \varepsilon
$$

Mas, D é reversive1; então, pe1a Proposição 2 (Capítulo 2) o passeio $c^{\prime}: q_{+}^{\prime} \longrightarrow q_{-}^{\prime}$ é o reverso de c em $D^{\prime}$ e

$$
\left(\varepsilon q_{+}^{\prime} s \varepsilon\right)|| c^{\prime} \|=\varepsilon q_{-}^{\prime} \varepsilon
$$

Por outro lado, como $s \in L=|B|$ e mais o fato de $B$ ser reversível e normalizada e de $C$ ser o acoplamento de $B$ com $B^{\prime}$, resulta pela Proposição 4 (Capítulo 2) que C tem um passeio $\mathrm{d}: \mathrm{z}_{-} \longrightarrow \mathrm{z}_{-}^{\prime}$ tal que

$$
\left(\varepsilon z_{-} s \varepsilon\right)|| d||=\varepsilon z_{-}^{\prime} s \varepsilon
$$

Utilizando-se da Proposição 5 (Capítulo 3) e pelas definições dos morfismos $f$ e g associados à máquina de Turing $S$, segue de (5), (6) e (7), respectivamente, que

$$
\begin{aligned}
\varepsilon q_{-} \varepsilon \#\left(\varepsilon q_{-} \varepsilon\right)[c] f=\left(\varepsilon q_{-} \varepsilon\right)[c] g \bar{H} \varepsilon q_{+} s \varepsilon, \\
\varepsilon q_{+}^{\prime} s \varepsilon \#\left(\varepsilon q_{+}^{\prime} s \varepsilon\right)\left[c^{\prime}\right] f=\left(\varepsilon q_{+}^{\prime} s \varepsilon\right)\left[c^{\prime}\right] g \bar{H} \varepsilon q_{-}^{\prime} \varepsilon \\
\\
\varepsilon z_{-} s \varepsilon \#\left(\varepsilon z_{-} s \varepsilon\right)[d] f=\left(\varepsilon z_{-} s \varepsilon\right)[d] g \bar{H}\left(\varepsilon z_{-}^{\prime} s \varepsilon\right) .
\end{aligned}
$$

Acs $\vdots$ h, pelas definições de $h_{1}$ e $h_{2}$, segue de (8), :yj e (10), respectivamente, que 


$$
\begin{gathered}
\left(\varepsilon q_{-} \varepsilon\right) h_{e} \phi \$ \# \phi \$\left(\varepsilon q_{-} \varepsilon\right)[c] h_{1}= \\
=\left(\varepsilon q_{-} \varepsilon\right)[c] h_{2} \phi \$ \bar{H} \phi \$\left(\varepsilon q_{+} s \varepsilon\right) h_{d} \\
\left(\varepsilon q_{+}^{\prime} s \varepsilon\right) h_{e} \phi \$ \# \phi \$\left(\varepsilon q_{+}^{\prime} s \varepsilon\right)\left[c^{\prime}\right] h_{1}= \\
=\left(\varepsilon q_{+}^{\prime} s \varepsilon\right)\left[c^{\prime}\right] h_{2} \phi \$ \bar{H} \phi \$\left(\varepsilon q_{-}^{\prime} \varepsilon\right) h_{d} \\
e \quad\left(\varepsilon z_{-} s \varepsilon\right) h_{e} \phi \$ H \phi \$\left(\varepsilon z_{-} s \varepsilon\right)[d] h_{1}= \\
=\left(\varepsilon z_{-} s \varepsilon\right)\left[d^{\prime}\right] h_{2} \phi \$ \bar{H} \phi \$\left(\varepsilon z_{-}^{\prime} s \varepsilon\right) h_{d} .
\end{gathered}
$$

Seja a seguinte palavra $\mathrm{w}^{\mathrm{em}} \mathrm{\Gamma}^{+}$,

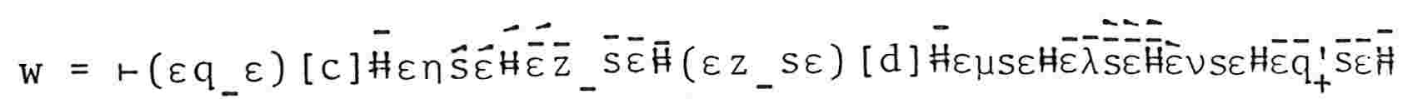

$$
\left(\varepsilon q_{+}^{\prime} s \varepsilon\right)\left[c^{\prime}\right]^{-1}
$$

Logo, resulta de (11), (12), (13) e das definições de $h_{1}$ e $h_{2}$ que $w h_{1}=w_{2}$ e para cada palavra $u$ em $\Gamma^{+}$, se $w=u v$, para algum $v$ em $\Gamma^{+}$, então $u h_{1} \neq u h_{2}$ (Observe a Figura 4. )

Além disso, pela definição de $h_{0}$ temos que $w_{0}=s$. Assim, cada palavra $s$ em $L$ pertence a $\left(e q\left(h_{1}, h_{2}\right)\right) h_{0}$. Portanto, $\mathrm{L} \subseteq\left(\mathrm{eq}\left(\mathrm{h}_{1}, \mathrm{~h}_{2}\right)\right) \mathrm{h}_{0}$. 


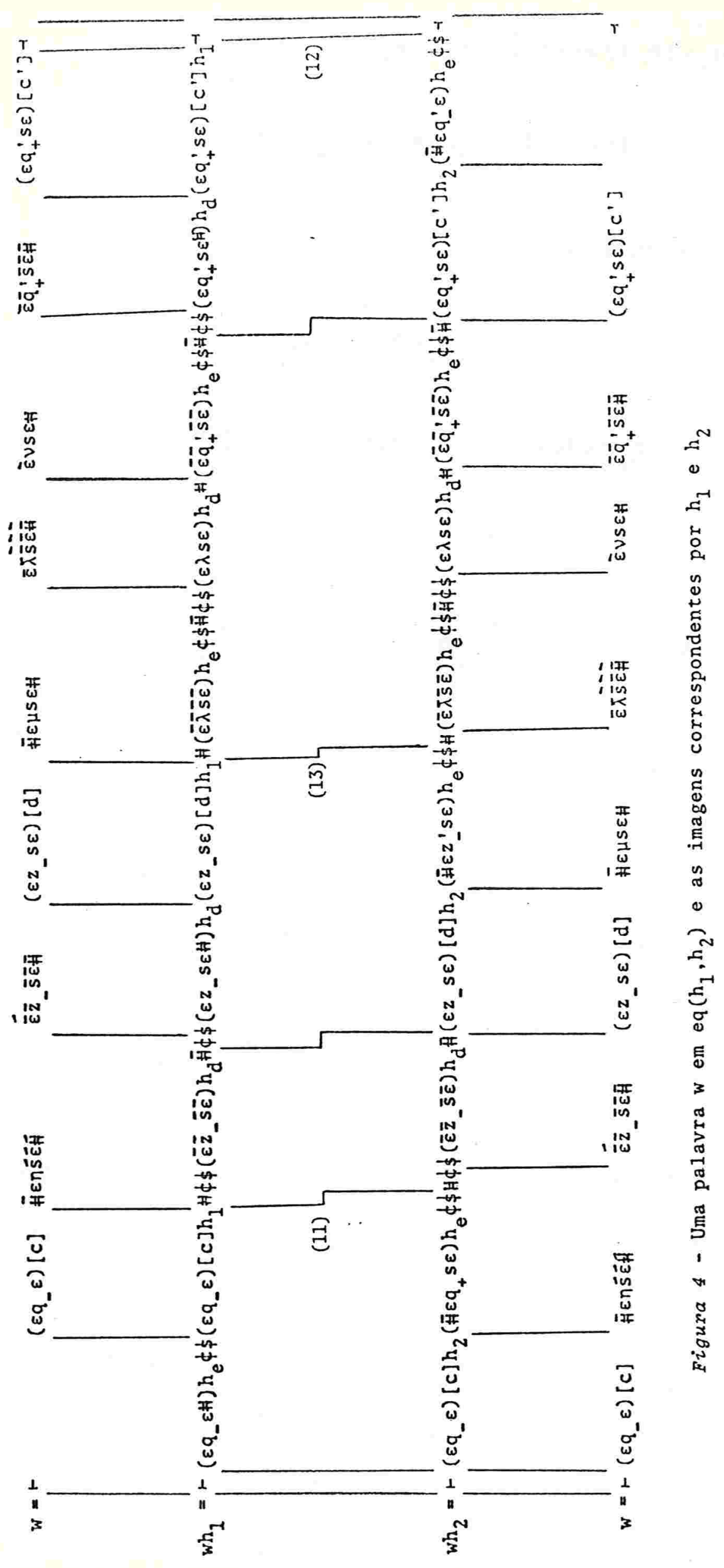


Demonstração do Lema 6

Seja w uma palavra em eq $\left(h_{1}, h_{2}\right)$.

Queremos provar que wh $_{0}$ pertence a L.

Como $w \in$ eq $\left(h_{1}, h_{2}\right)$, wh $h_{1}=w_{2}$ e para cada u em $\Gamma^{+}$, se $w=u v$, para algum $v$ em $\Gamma^{+}$, então $u h_{1} \neq u h_{2}$.

Mas, $|w| \geq 2$, pois, para cada $\gamma$ em $\Gamma, \gamma h_{1} \neq \gamma h_{2}$.

Alêm disso, $\vdash(-1)$ é o ünico elemento de $\Gamma$ tal que $\vdash h_{1}$ e $\vdash h_{2}$ $\left(t_{1}\right.$ e $\left.\dashv h_{2}\right)$ são ambos segmentos iniciais (finais) de uma mesma palavra. Logo, $w=w_{1}^{-1}$, para algum $w_{1}$ em $\Gamma^{+}, \quad j a ́$ que $(\vdash-1) h_{1} \neq(r-1) h_{2}$. Podemos supor ainda que nenhum dos dois sím bolos, rou $t$, ocorre em $w_{1}$, jä que w $\in$ eq $\left(h_{1}, h_{2}\right)$.

A seguir, vamos definir dois morfismos que nos aux $\underline{i}$ liarão a relacionar os morfismos $h_{1}$ e $h_{2}$ com os morfismos $f_{1}$ e $g_{1}$, respectivamente.

Já vimos que os morfismos $f_{1}$ e $g_{1}: \Gamma_{2}^{*} \longrightarrow \Delta_{2}^{*}$ são extensões dos morfismos associados à máquina de Turing $S$ e que os morfismos $h_{1}$ e $h_{2}$ estão definidos sobre $\Gamma^{*}$ com valores em $\Delta^{*}$, sendo que

e

$$
\Gamma=\Gamma_{2} U \overline{\bar{\Sigma}} U \overline{\bar{\Sigma}} U\{\bar{\varepsilon}, \overline{\#}, \overline{\bar{\varepsilon}}, \bar{\varepsilon}, \overline{\bar{\varepsilon}}, \overline{\overline{\#}}\}
$$

$\Delta=\Delta_{2} U\{\phi, \$\}$

Seja $h_{\Delta_{2}}: \Delta^{*} \longrightarrow \Delta_{2}^{*}$ o morfismo que preserva $\Delta_{2}$, isto é, 


$$
\begin{aligned}
& \phi \mathrm{h}_{\Delta_{2}}=\$ \mathrm{~h}_{\Delta_{2}}=1 \\
& \delta \mathrm{h}_{\Delta_{2}}=\delta \text {, para cada } \delta \text { em } \Delta \backslash\{\phi, \$\}=\Delta_{2} .
\end{aligned}
$$

e

Seja a: $\Gamma^{*} \longrightarrow \Gamma_{2}^{*}$ um morfismo dado por

$$
\begin{aligned}
& \gamma a=\gamma \text {, para cada } \gamma \text { em } \Gamma_{2} ; \\
& \text { se } \gamma=\bar{\sigma} \text {, para } \sigma \text { em } \Sigma \cup\{\varepsilon, \#\} \text {, então } \gamma a=\sigma ; \\
& \text { se } \gamma=\bar{\varepsilon} \text {, então } \gamma a=\bar{\varepsilon} ; \\
& \text { se } \gamma=\bar{\varepsilon} \text {, então } \gamma a=\varepsilon \\
& \text { e se } \gamma=\overline{\bar{\sigma}} \text {, para } \sigma \text { em } \Sigma U\{\varepsilon, \#\} \text {, então } \gamma a=\bar{\sigma} .
\end{aligned}
$$

Assim, è fácil verificar que o seguinte diagrama é comutativo, isto é, $h_{1} h_{\Delta_{2}}=a f_{1}$ e $h_{2} h_{\Delta_{2}}=a g_{1}$.

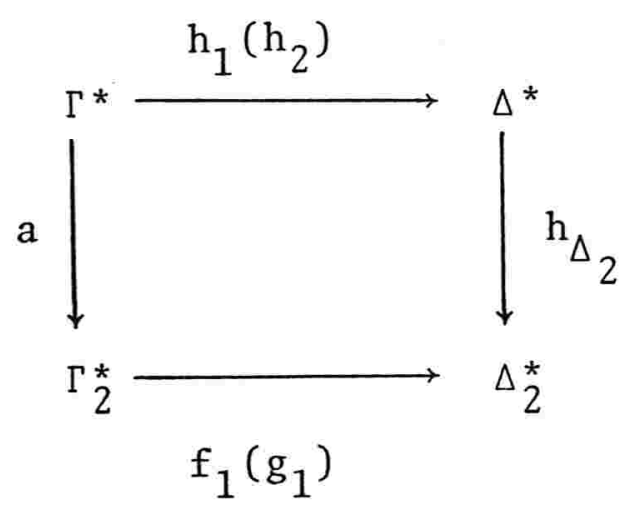

Seja ainda $h_{A_{S}}: \Gamma^{*} \longrightarrow\left(A_{S}\right)^{*}$ o morfismo que preserva $A_{S}$

Retornando à demonstração, de $w h_{1}=w_{2}$ segue que

$$
\left(\vdash w_{1}-1\right) h_{1}=\left(1 w_{1}-1\right) h_{2} \cdot
$$


Então, $\left(1-w_{1}^{-1}\right) h_{1} h_{\Delta_{2}}=\left(1-w_{1}^{-1}\right) h_{2} h_{\Delta_{2}} ;$ o que implical que

$$
\left(1-w_{1}^{-1) a f_{1}}=\left(1-w_{1}-1\right) a g_{1} .\right.
$$

$\log 0$,

$$
\vdash \varepsilon q_{-} \varepsilon \#\left(w_{1} a_{1}\right) \dashv=\vdash\left(w_{1} a g_{1}\right) \bar{H} \varepsilon q_{-}^{\prime} \varepsilon \dashv \text {. }
$$

Portanto,

$$
\varepsilon q_{-} \varepsilon \#\left(w_{1} a\right) f_{1-}=\left(w_{1} a\right) g_{1} b H \varepsilon q_{-}^{\prime} \varepsilon .
$$

(Lembremos que $\underline{b}$ é o morfismo que "elimina" a "barra".)

$$
\text { Assim, pela Proposição } 6 \text { (Capítulo 3) existe um }
$$
passeio c em $S$ tal que.

$$
\left(\varepsilon q_{-} \varepsilon\right)|| c||=\varepsilon q_{-}^{\prime} \varepsilon \quad \text { e } \quad w_{1} h_{A_{S}}=c .
$$

Como o passeio c tem origem em q_ e têrmino em q⿳, segue, por construção da máquina de Turing $S$, que existem passeios não degenerados

$$
\begin{array}{ll}
c_{1}: q_{-} \longrightarrow q_{+} \text {em } & 0, \\
c_{2}: z_{-} \longrightarrow & z_{-}^{\prime} \text { em } c, \\
\text { e } & c_{1}^{\prime}: q_{+}^{\prime} \longrightarrow q_{-}^{\prime} \text { em } D^{\prime},
\end{array}
$$

onde $c_{1}^{\prime}$ é o reverso de $c_{1}$, tais que

$$
c=c_{1} n c_{2} \mu \nu c_{1}^{\prime} .
$$


Mas, como $w_{1} h_{A_{S}}=c$, existem palavras $w_{2}, w_{3}, w_{4}$ e $w_{5}$ em $\Gamma^{*}$ tais que

$$
\begin{aligned}
& w_{1}=w_{2} n w_{3} \mu w_{4} \nu w_{5}, \\
& w_{2} h_{A_{S}}=c_{1}, \\
& w_{3} h_{A_{S}}=c_{2}, \\
& w_{4} h_{A_{S}}=1 \\
& w_{5} h_{A_{S}}=c_{1}^{\prime} .
\end{aligned}
$$

e

Alēm disso, $w_{2} \in(\Gamma a)^{+}=\Gamma_{2}^{+}$.

De fato, suponhamos por absurdo que exista um símbolo $\sigma$ em $\Sigma U\{\varepsilon, H\}$ tal que $w_{2}=x \bar{\sigma} y$, para algum $x$ em $(\Gamma a) *$ e algum $y$ em $\Gamma^{*}$. Então, pelas definições de $h_{1}$ e $h_{2}$ existe uma palavra $z$ em $\Delta^{+}$tal que

$$
\begin{array}{r}
(r x) h_{1}=(1-x) h_{2} z \quad \text { e } \quad z=\$ \$\left(z_{1} h_{d}\right), \\
\text { em } \Delta_{2}^{+} .
\end{array}
$$

Logo, de (14) e (17) segue que

$$
z\left(\bar{\sigma}_{y \eta w_{3}} \mu w_{4} \nu w_{5}^{-1}\right) h_{1}=\left(\tilde{\sigma}_{y n w_{3}} \mu w_{4} \nu w_{5}{ }^{-1}\right) h_{2} .
$$

Então,

$\phi s\left(z_{1} h_{d}\right) \$ \bar{\sigma} \phi\left(y \eta w_{3} \mu w_{4} v w_{5}^{-1}\right) h_{1}=\$ \sigma \phi\left(y n w_{3} \mu w_{4} \nu w_{5}^{-1}\right) h_{2}$, 
o que è um absurdo.

Analogamente, chegamos a um absurdo se $w_{2}=x \sigma y, c o m$ $\mathrm{x}$ em $(\Gamma \mathrm{a})^{*}, \sigma \mathrm{em}\{\overline{\bar{\varepsilon}}, \bar{\varepsilon}, \overline{\bar{\varepsilon}}, \overline{\bar{H}}\} \quad \mathrm{U} \overline{\bar{\Sigma}}$ e y em $\Gamma^{*}$.Portanto, $\mathrm{w}_{2} \in(\Gamma \mathrm{a})^{+}$.

Assim, pelas definições de $h_{1}$ e $h_{2}$ e de (14) e (17), existe uma palavra $v_{1}$ em $\Delta_{2}^{+}$tal que

$$
\begin{aligned}
& \left(\vdash w_{2}\right) h_{1}=\left(\vdash w_{2}\right) h_{2}\left(v_{1} h_{e}\right) \phi \$ \\
\Longrightarrow & \left(\vdash w_{2}\right) h_{1} h_{\Delta_{2}}=\left(\vdash w_{2}\right) h_{2} h_{\Delta_{2}} v_{1} \\
\Longrightarrow & \left(\vdash w_{2}\right) a f_{1}=\left(\vdash w_{2}\right) a g_{1} v_{1} \\
\Longrightarrow & \vdash \varepsilon q_{-} \varepsilon \#\left(w_{2} f_{1}\right)=\vdash\left(w_{2} g_{1}\right) v_{1} \\
\Longrightarrow & \varepsilon q_{-} \varepsilon \#\left(w_{2} f_{1} b\right)=\left(w_{2} g_{1} \underline{b}\right)\left(v_{1} \underline{b}\right) .
\end{aligned}
$$

Então, pela Proposição 6 (Capítulo 3) existem palavras $s_{1}$ e $t_{1}$ em $\left(\Delta_{2} \underline{b} \backslash\{\#\}\right) *$ tais que

$$
\begin{aligned}
& v_{1} \underline{b}=s_{1}^{\# t_{1}}, \\
& t_{1} s_{1} \text { é uma configuração instantânea de } s
\end{aligned}
$$

e existe um passeio $r_{1}$ em $S$ tal que

$$
\left(\varepsilon \mathrm{q}_{-} \varepsilon\right)|| \mathrm{r}_{1}||=\mathrm{t}_{1} \mathrm{~s}_{1} \text { e } \mathrm{r}_{1}=\mathrm{w}_{2} \mathrm{~h}_{\mathrm{A}_{\mathrm{S}}} \text {. }
$$


Mas, de (15) e (18) resulta que $r_{1}=c_{1}$ é um passeio de $q_{-}$a $q_{+} \operatorname{cm} D$

Logo, por construção da māquina $D$, concluímos que

$$
\mathrm{t}_{1} \mathrm{~s}_{1}=\varepsilon \mathrm{q}_{+} \mathrm{s} \varepsilon \text {, para alguma palavra } \mathrm{s} \text { em } \Sigma^{*} \text {. }
$$

Por outro lado, como $\left(\vdash w_{1}^{\dashv}\right) h_{1}=\left(\vdash w_{1} \dashv\right) h_{2}$ segue de (17) e (21) que

$$
\left(v_{1} h_{e} \underline{b}\right) \notin \$\left(n w_{3} \mu w_{4} v w_{5}^{-1}\right) h_{1} \underline{b}=\left(n w_{3} \mu w_{4} v w_{5}^{-1}\right) h_{2} \underline{b} .
$$

E, utilizando (22) e as definições de $h_{1}$ e $h_{2}$ temos que

$$
\phi \$\left(s_{1} h_{d}\right) \# \phi \$\left(t_{1} h_{d}\right) z_{-} \phi\left(w_{3} \mu w_{4} v w_{5}{ }^{-1}\right) h_{1} b=\phi \$ q_{+} \phi\left(w_{3} \mu w_{4} v w_{5}-1\right) h_{2} \underline{b} .
$$

Então, de (23) $s_{1}=q_{+} s \varepsilon$ e $t_{1}=\varepsilon$. Logo,

$$
\$(s \varepsilon) h_{d^{\#} \phi \$ \varepsilon \phi \$ z_{-}} \phi\left(w_{3} \mu w_{4} \nu w_{5}{ }^{-1}\right) h_{1} \underline{b}=\left(w_{3} \mu w_{4} v w_{5}^{-1}\right) h_{2} \underline{b} .
$$

Como $\mu h_{2} \underline{b}=\$ \$ z_{-}^{\prime}$ e $z_{-}^{\prime} \in Q_{S}$, segue que

$$
w_{3} h_{2} \underline{b}=\$(s \varepsilon) h_{d} \# \phi \$ \varepsilon \phi \$ z_{-}\left(y_{3} h_{2}{ }^{b}\right)
$$

para algum $y_{3}$ em $\Gamma^{*}$.

Então, pela definição de $h_{2}$, temos que

$$
\mathrm{w}_{3} \mathrm{~b}_{-}=\overrightarrow{\mathrm{s}} \overrightarrow{\bar{E}} \vec{\varepsilon} \mathrm{z}_{-}\left(\mathrm{y}_{3} \mathrm{~b}_{-}\right)
$$

E, de (24), (25), (26) e da definição de $h_{1}$ segue que 


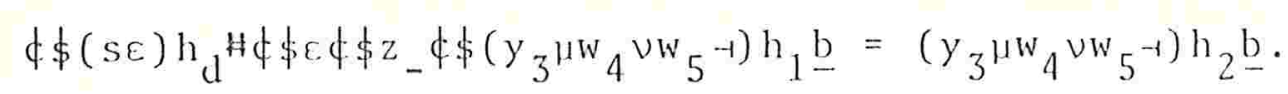

E, analogamente, obtemos que

$$
y_{3} h_{2} \underline{b}=\$ \$(s \varepsilon) h_{d} \#\left(u_{3} h_{2} \underline{b}\right) \text {, para algum } u_{3} \mathrm{em} \Gamma \text { * }
$$

e, conseqüentemente,

$$
\mathrm{y}_{3-} \underline{\mathrm{b}}=\mathrm{sEH}\left(\mathrm{u}_{3} \underline{\mathrm{b}}\right)
$$

Então, de (27), (28), (29) e da definição de $\mathrm{h}_{1}$ segue que

$$
\phi \$ \varepsilon \phi \$ z_{-} \phi \$(s \varepsilon) h_{d} \# \phi \$\left(u_{3} \mu w_{4} v w_{5}^{-1}\right) h_{1} \underline{b}=\left(u_{3} \mu w_{4} \nu w_{5}-1\right) h_{2} \underline{b} .
$$

Além disso, podemos verificar sem dificuldades que $u_{3}$ também pertence a $(\Gamma \mathrm{a}){ }^{*}$.

Assim, pelas definições de $h_{1}$ e $h_{2}$, segue de (30) que existe uma palavra $v_{2}$ em $\Delta_{2}^{+}$tal que

$$
\begin{aligned}
& \phi \$ \varepsilon \phi \$ z_{-} \phi \$(s \varepsilon) h_{d} \# \phi \$\left(u_{3} h_{1} \underline{b}\right)=\left(u_{3} h_{2} \underline{b}\right)\left(v_{2} h_{e} \underline{b}\right) \phi \$ \\
\Longrightarrow & \varepsilon z_{-} s \varepsilon \#\left(u_{3} h_{1} h_{\Delta_{2}}\right) \underline{b}=\left(u_{3} h_{2} h_{\Delta_{2}}\right) \underline{b}\left(v_{2} \underline{b}\right) \\
\Longrightarrow & \varepsilon z_{-} s \varepsilon \#\left(u_{3} a f_{1}\right) \underline{b}=\left(u_{3} a g_{1}\right) \underline{b}\left(v_{2} \underline{b}\right) \\
\Longrightarrow & \varepsilon z_{-} s \varepsilon \#\left(u_{3} f_{1} b\right)=\left(u_{3} g_{1} \underline{b}\right)\left(v_{2} \underline{b}\right) .
\end{aligned}
$$

Então pela Proposição 6 (Capítulo 3) existem pala- 
$\operatorname{vras} s_{2}$ e $t_{2} \operatorname{cm}\left(\Delta_{2}-b \backslash\{\#\}\right) *$ tais que

$$
\begin{aligned}
& v_{2} \underline{b}=\mathrm{s}_{2} \# \mathrm{t}_{2}, \\
& \mathrm{t}_{2} \mathrm{~s}_{2} \text { è uma configuração instantânea de } s
\end{aligned}
$$

e existe um passeio $r_{2}$ em $S$ tal que

$$
\left(\varepsilon z_{-} s \varepsilon\right)|| r_{2}||=t_{2} s_{2} \text { e } r_{2}=u_{3} h_{A_{S}} \text {. }
$$

Mas, de (26) e (29) temos que

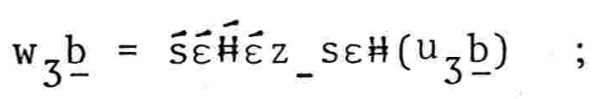

então $w_{3} h_{A_{S}}=u_{3} h_{A_{S}}$.

Logo, de (16) e (19) vem que $\mathrm{r}_{2}=\mathrm{c}_{2}$ é um passeio de $z_{-} z_{\text {_ }}^{\prime}$ em C. Então, por construção da máquina de Turing $C$ existem passeios

$$
\begin{gathered}
\mathrm{d}: z_{-} \longrightarrow z_{+} \text {em } B, \text { onde }\left\{z_{+}\right\}=F_{B} \\
\text { e } \quad d^{\prime}: z_{+}^{\prime} \longrightarrow z_{-}^{\prime} \text { em } B^{\prime} \text {, reverso de } d
\end{gathered}
$$$$
\text { tais que } c_{2}=\mathrm{d} \alpha \mathrm{d}^{\prime}, \operatorname{com} \alpha=\left(\mathrm{z}_{+}, \mathrm{N}, \mathrm{V}, \mathrm{z}_{+}^{\prime}\right)
$$$$
\text { Então, de }\left(\varepsilon z_{-} s \varepsilon\right)|| c_{2}||=t_{2} s_{2} \text { segue que }
$$$$
\left(\varepsilon z_{-} s \varepsilon\right)|| d|||| \alpha|||| d^{\prime}||=t_{2} s_{2} \cdot
$$

Logo, existem palavras $\ell_{1} \mathrm{e} \ell_{2}$ em $\mathrm{T}_{\mathrm{C}}^{*}$ tais que 


$$
\begin{aligned}
& \left(\varepsilon z_{-} \mathrm{S} \varepsilon\right)|| d||=\varepsilon l_{1} z_{+} \ell_{2} \varepsilon, \\
& \left(\varepsilon l_{1} z_{+} \ell_{2} \varepsilon\right)|| \alpha||=\varepsilon \ell_{1} z_{+}^{\prime} \varepsilon \text { c } \quad \ell_{2}=1
\end{aligned}
$$

e, utilizando a Proposição 2 (Capítulo 2) temos que

$$
\left(\varepsilon l_{1} z_{+}^{\prime} \varepsilon\right)|| d^{\prime}||=\varepsilon z_{-}^{\prime} s \varepsilon
$$

Portanto,

$$
\begin{aligned}
& s \in|B|=|A|=L \\
& \text { Além disso, resulta que } t_{2} s_{2}=\varepsilon z_{-}^{\prime} s \varepsilon \\
& \text { Assim, de }(30) \text { e (31) obtemos que } \\
& \left(v_{2} h_{e} \underline{b}^{-} \phi \$\left(\mu w_{4} \nu w_{5}-1\right) h_{1} \underline{b}=\left(\mu w_{4} \nu w_{5}^{-1}\right) h_{2} \underline{b}\right. \text {. } \\
& \text { Então, utilizando (32) e as definições de } h_{1} \text { e } h_{2}
\end{aligned}
$$

vem que

$$
\phi \$\left(s_{2} h_{d}\right) \# \phi \$\left(t_{2} h_{d}\right) \lambda \phi \$\left(w_{4} v w_{5}-1\right) h_{1} b_{-}=\phi \$ z_{-}^{\prime}\left(w_{4} v w_{5}-1\right) h_{2} \underline{b} .
$$

E de (34) segue que $s_{2}=z_{-}^{\prime} s \varepsilon$ e $t_{2}=\varepsilon$. Logo,

$$
\phi \$(s \varepsilon) h_{\mathrm{d}} \# \phi \$ \varepsilon \phi \$ \lambda \phi \$\left(\mathrm{w}_{4} \nu \mathrm{w}_{5}-1\right) \mathrm{h}_{1} \underline{\mathrm{b}}=\left(\mathrm{w}_{4} \nu \mathrm{w}_{5}{ }^{-1}\right) \mathrm{h}_{2} \underline{\mathrm{b}} .
$$

Examinando-se a definição de $h_{2}$ temos que $\mathrm{w}_{4} \mathrm{~h}_{2} \underline{\mathrm{b}}=(\mathrm{s} \varepsilon) \mathrm{h}_{\mathrm{e}} \phi \$ \hbar \phi \$ \varepsilon \phi \$ \lambda \phi \$\left(\mathrm{y}_{4} \mathrm{~h}_{2} \mathrm{~b}\right)$, para algum $\mathrm{y}_{4}$ em $\Gamma^{*}$ 
c $\quad w_{4} \underline{b}=\operatorname{seHE} \lambda\left(y_{4} \underline{b}\right)$.

Então, de (35), (36) e da definição de $h_{1}$ segue que

$$
(\mathrm{s} \varepsilon) \mathrm{h}_{\mathrm{d}} \# \phi \$ \varepsilon \phi \$ \lambda\left(y_{4} \nu \mathrm{w}_{5}{ }^{-1}\right) \mathrm{h}_{1} \underline{\mathrm{b}}=\left(\mathrm{y}_{4} \nu \mathrm{w}_{5}-1\right) \mathrm{h}_{2} \mathrm{~b} .
$$

E, novamente, examinando-se a definição de $\mathrm{h}_{2} \mathrm{e}$

$$
\begin{array}{ll}
(20) \text { e (36) resulta que } \\
\\
\left(y_{4} \nu\right) h_{2} \underline{b}=(s \varepsilon) h_{d} \# \phi \$ \varepsilon \phi \$ \lambda \\
\text { e } & y_{4} \underline{b}=\bar{s} \bar{\varepsilon} \overline{\#} \bar{\varepsilon} .
\end{array}
$$

Assim, usando (37) e a definição de $h_{1}$ temos que

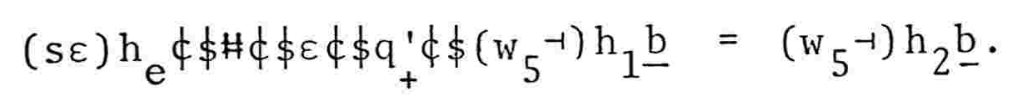

$$
\text { Como } \rightarrow h_{2} \underline{b}=\$ \$ \hbar \phi \$ \varepsilon \phi \$ q_{-}^{\prime} \phi \$ \varepsilon \phi \$ \dashv \text {, segue que, para a1- }
$$
$\operatorname{gum} \mathrm{y}_{5}$ em $\Gamma^{*}$,

$$
w_{5} h_{2} \underline{b}=(s \varepsilon) h_{e} \phi \$ H\left(y_{5} h_{2} \underline{b}\right) .
$$

$E$, pela definição de $h_{2}$ temos que

$$
w_{5} \underline{b}=s \varepsilon \#\left(y_{5} b\right) \text {. }
$$

Logo, de (39) e da definição de $h_{1}$ segue que

$$
\phi \$ \varepsilon \phi \$ q_{+}^{\prime} \phi \$(s \varepsilon) h_{d} \# \phi \$\left(y_{5}-1\right) h_{1} \underline{b}=\left(y_{5}-1\right) h_{2} b .
$$


Além disso, podemos verificar que $y_{5} \in$ ( $\Gamma$ a ${ }^{*}$.

$$
\text { Portanto, de (14), (17), (26), (29), (36), (38) c }
$$

(40), concluímos que

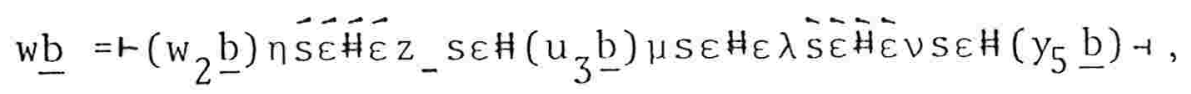

com $w_{2}, u_{3}$ e $y_{5}$ em (Га)*.

Então, pela definição do morfismo $h_{0}$ e de (33) segue que $w_{0}=s \in L$. Logo, $\left(e q\left(h_{1}, h_{2}\right)\right) h_{0} \subseteq L$.

2. UMA REPRESENTACÃO DE LINGUAGENS RECURSIVAMENTE ENUMERĀVEIS ATRAVES DE CODIGOS DE ATRASO UM EM SENTIDOS OPOSTOS E PERTENCENTES A PS.

Podemos demonstrar que os morfismos $h_{1}$ e $h_{2}$ construí dos na demonstração do Teorema 3 pertencem a PS.

Assim, obtemos um resultado mais forte que apresentamos a seguir.

TEOREMA 4 - Para cada linguagem recursivamente enumeráve1 $\mathrm{L} \subseteq \Sigma^{*}$, existem efetivamente alfabetos $\Gamma$ e $\Delta$, um morfismo $\mathrm{h}_{0}: \Gamma^{*} \longrightarrow \Sigma^{*}$ e códigos $\mathrm{h}_{1}, \mathrm{~h}_{2}: \Gamma^{*} \longrightarrow \Delta^{*}$ pertencentes a PS com $h_{1}\left(h_{2}\right)$ de atraso um da esquerda para a direita (da direita para a esquerda) tais que $L=\left(e q\left(h_{1}, h_{2}\right)\right) h_{0}$.

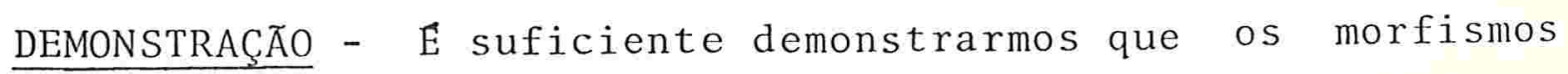


$\mathrm{h}_{1}, \mathrm{~h}_{2}: \mathrm{I}^{*} \longrightarrow \Delta^{*}$, construídos na demonstração do Teorema 3,per tencem a PS.

Inicialmente, para mostrarmos que o morfismo $\mathrm{h}_{1}$ per tence a PS, consideremos o alfabeto

$\Xi_{1}=\mathrm{T}_{S} \cup \mathrm{Q}_{S} \cup \overline{\mathrm{T}}_{S} \cup \bar{Q}_{1} \cup \overline{\mathrm{K}}_{1} \cup \overline{\mathrm{K}}_{2} \cup \overline{\bar{\Sigma}} \cup\{n\} \cup\{*,-,-1\} \cup\{\varepsilon, \#, \bar{\varepsilon}, \overline{\bar{H}}, \overline{\bar{\varepsilon}}, \overline{\bar{H}}, \bar{\varepsilon}\}$, onde $Q_{1}=\left\{r \in Q_{S}\right.$ /nas existem em $S$ arestas com término em $r$ e rótulo $\mathrm{E}_{\sigma}^{-1} \times \mathrm{N}$, para algum $\sigma$ em $\mathrm{T}_{S}$ ou $\left.\mathrm{V} \times \mathrm{N}\right\}$,

$K_{1}=\left\{(\sigma, r) \in T_{S} \times Q_{S} /\right.$ existem em $S$ arestas com tërmino em $\mathrm{r}$ e rótulo $\left.\mathrm{E}_{\sigma}^{-1} \times \mathrm{N}\right\}$,

$K_{2}=\left\{(\varepsilon, r) \in \varepsilon \times Q_{S}\right.$ lexistem em $S$ arestas com término em $\mathrm{r}$ e rótulo $\mathrm{V} \times \mathrm{N}\}$

$e^{*}$ é um novo símbolo.

Sejam os morfismos

$$
\mathrm{P}_{1}: \Gamma^{*} \longrightarrow \mathrm{E}_{1}^{*} \quad \text { e } \quad \mathrm{s}_{1}: \Xi_{1}^{*} \longrightarrow \Delta^{*},
$$

definidos pela Tabela 4 onde $\sigma$ denota um símbolo em $\mathrm{T}_{S} ; \mathrm{q}, \mathrm{r}, \lambda$, $z_{-}, z_{-}^{\prime}, q_{+}$e $q_{+}^{\prime}$ são elementos de $Q_{S} ;(q, i, j, r)$, com $i \in E_{S}$ e $j \in D_{S}$, representa uma aresta em $A_{S}$ e $(\sigma, r)$ e $(\varepsilon, r)$ são ele mentos de $\mathrm{K}_{1}$ e $\mathrm{K}_{2}$, respectivamente.

Pode-se verificar que o morfismo $\mathrm{p}_{1}\left(\mathrm{~s}_{1}\right)$ é um prefixo (sufixo).

Além disso, podemos observar que 


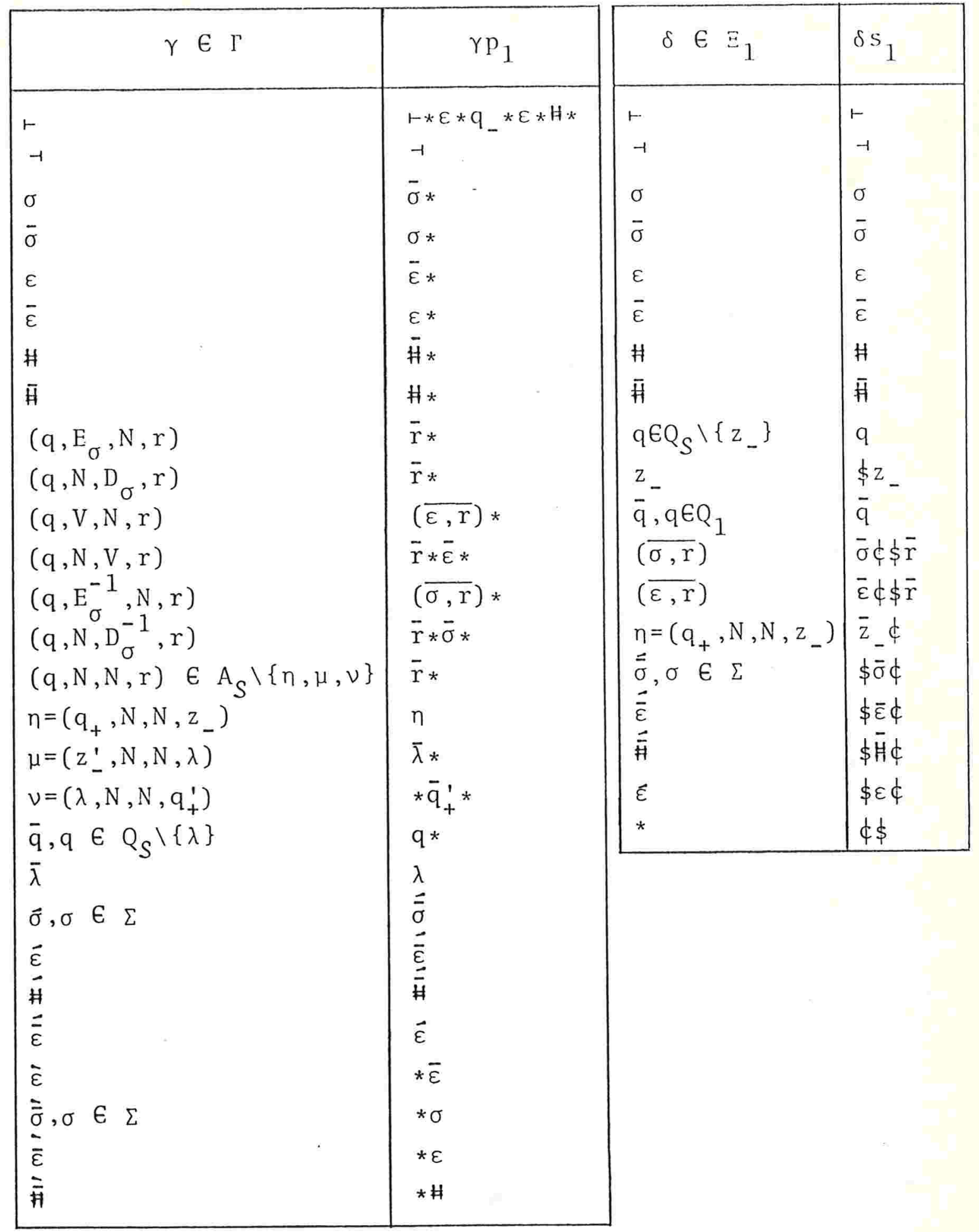

Figura 4-Definição dos morfismos $\mathrm{p}_{1}$ e $\mathrm{s}_{1}$ associados a $\mathrm{h}_{1}$. 
(i) a aresta $\mu=\left(z_{-}^{\prime}, N, N, \lambda\right)$ é a ünica aresta de $S$ com término em $\lambda$ e tem rótulo $N \times N$; então $\lambda \in Q_{1}$;

(ii) construímos a máquina de Turing $S$, mais precisamente $D$, de modo que a única aresta de $D$ com origem em $q_{+}$tivesse rótulo $N \times N$. Então, $D^{\prime}$ tem apenas uma aresta com término em $q_{+}^{\prime}$ e esta aresta tem rótulo $\mathrm{N} \times \mathrm{N}$; isto implica que $q_{+}^{\prime} \in Q_{1}$.

Assim, de (i), (ii) e pelas definições de $h_{1}, p_{1}$ $\mathrm{s}_{1}$, é fácil ver que $\mathrm{h}_{1}=\mathrm{p}_{1} \mathrm{~s}_{1}$. Portanto, $\mathrm{h}_{1}$ pertence a PS.

A fim de mostrarmos que o morfismo $h_{2}$ também perten ce a PS, consideremos o alfabeto

$$
\Xi_{2}=T_{S} \cup \bar{T}_{S} \cup \bar{Q}_{S} \cup Q_{2} \cup K_{3} \cup K_{4} \cup \bar{\Sigma} \cup\{\eta\} \cup\left\{{ }^{*}, \vdash,-1\right\} \cup\{\varepsilon, H, \bar{\varepsilon}, \overline{\#}, \bar{\varepsilon}, \overline{\#}, \bar{\varepsilon}\},
$$

onde $Q_{2}=\left\{q \in Q_{S} /\right.$ não existem em $S$ arestas com origem em $q$ e rótulo $\mathrm{E}_{\sigma} \times \mathrm{N}$, para algum $\sigma \mathrm{em}_{S}$ ou $\left.\mathrm{V} \times \mathrm{N}\right\}$,

$$
\begin{gathered}
K_{3}=\left\{(\sigma, q) \in T_{S} \times Q_{S} / \text { existem em } S\right. \text { arestas com origem em } \\
\left.q \text { e rótulo } E_{\sigma} \times N\right\}
\end{gathered}
$$

e $\quad \mathrm{K}_{4}=\left\{(\varepsilon, q) \in \varepsilon \times Q_{S} /\right.$ existem em $S$ arestas com origem em $\mathrm{q}$ e rótulo $\mathrm{V} \times \mathrm{N}\}$.

Sejam os morfismos

$$
\mathrm{p}_{2}: \Gamma^{*} \longrightarrow \Xi_{2}^{*} \text { e } \quad \mathrm{s}_{2}: \Xi_{2}^{*} \longrightarrow \Delta^{*}
$$


definidos pela rabela 5 , onde o denota um símbolo $\mathrm{cm}_{\mathrm{S}} ; q, \mathrm{q}$, $\lambda, z_{-}, z_{-}^{\prime}, q_{+}$e $q_{+}^{\prime}$ são elementos de $Q_{S} ;(q, i, j, r)$, com i $\mathrm{cm} \mathrm{E}_{S}$ e $\mathrm{j} \mathrm{em} \mathrm{D}_{S}$, representa uma aresta em $\wedge_{S}$ e $(\sigma, q)$ e $(\varepsilon, q)$ são elementos de $K_{3}$ e $K_{4}$, respectivamente.

Pode-se verificar tambëm que o morfismo $\mathrm{p}_{2}\left(\mathrm{~s}_{2}\right)$ é um prefixo (sufixo). Além disso, podemos observar que a aresta $\mu=\left(z_{-}^{\prime}, N, N, \lambda\right)$ è a únjca aresta de $S$ com origem em $z_{-}^{\prime}$ e tem rótulo $N \times N$; então $z_{-}^{\prime} \in Q_{2}$. Analogamente para a aresta $\nu=\left(\lambda, N, N, q_{+}^{\prime}\right)$; então $\lambda \in Q_{2}$.

Portanto, pelas observações acima e pelas definições de $h_{2}, p_{2}$ e $s_{2}$, é fáci.l ver que $h_{2}=p_{2} s_{2}$. Assim, $h_{2}$ pertence a PS.

Logo, complètamos a demonstração do Teorema 4.

3. OUTRAS REPRESENTAÇÕES DE LINGUAGENS RECURSIVAMENTE ENUMERAVEIS

Nesta seção apresentamos algumas outras representações de linguagens recursivamente enumeräveis, representações estas feitas em termos de outras linguagens mais simples.

Inicialmente, consideramos um dos primeiros resultados desse tipo obtido para linguagens livres de contexto. 


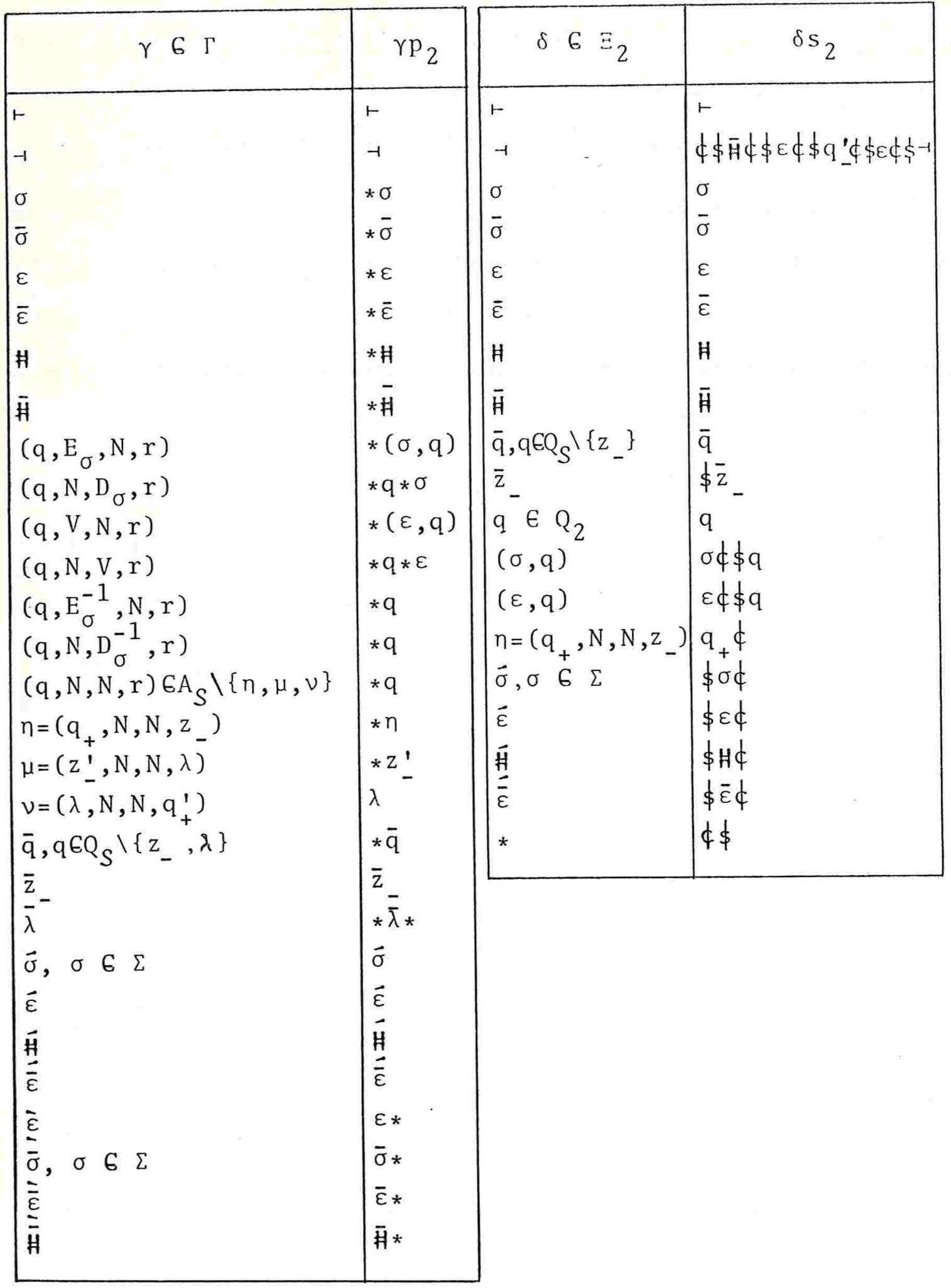

Tabela 5 - Definição dos morfismos $\mathrm{p}_{2}$ e $\mathrm{s}_{2}$ associados a $\mathrm{h}_{2}$ 
TEOREMA 5 - Chomsky e Schutzenberger

Para cada linguagem livre de contexto L existem uma linguagem de Dyck D, uma linguagem regular $R$ e um morfismo h tais que $L=(D \cap R) h$.

$\mathrm{Na}$ demonstração desse teorema a linguagem $\mathrm{D}$ depende de L. Então, obteve-se uma versão mais forte desse teorema, considerando-se uma linguagem de Dyck que dependa apenas do alfabeto $\Sigma$ tal que $L \subseteq \Sigma^{*}$.

\section{TEOREMA 6 -}

Seja $\sum$ um alfabeto com n letras.Existem um alfabeto $\Gamma$ com $2 n+4$ letras, uma linguagem de Dyck D sobre $\Gamma$ e um morfismo $\mathrm{h}$ de $\Gamma^{*}$ em $\Sigma^{*}$ tais que para cada linguagem livre de contexto $L$ sobre $\Gamma$ existe uma linguagem regular $R$ sobre $\Gamma$ com a propriedade $L=(D \cap R) h$.

Com relação às linguagens recursivamente enumeráveis, Salomaa (1973-Capítulo III) estabeleceu alguns resultados ne se sentido, os quais apresentamos a seguir.

\section{TEOREMA 7 - Salomaa}

Para cada linguagem recursivamente enumeräve1 L, existem linguagens livres de contexto $L_{1}$ e $L_{2}$ e um morfismo $h$ tais que $L=\left(L_{1} \cap L_{2}\right) h$. 
Nesse caso, as linguagens $\mathrm{L}_{1}$ e $\mathrm{L}_{2}$ e o morfismo h dependem da linguagem L. Esse resultado foi fortalecido conside rando-se as 1 inguagens $\mathrm{L}_{1}$ e $\mathrm{L}_{2}$ e o morfismo h dependendo apenas do alfabeto $\Sigma$ tal que $L \subseteq \Sigma^{*}$.

TEOREMA $8-$ Salomaa

Para cada 1 inguagem recursivamente enumerável $L \subseteq \Sigma^{*}$, existem linguagens 1 ivres de contexto $L_{1}$ e $L_{2}$ sobre $\bar{\Sigma} U\{a, b\}$ e um morfismo $h:(\bar{\Sigma} \cup\{a, b\}) * \longrightarrow \Sigma^{*}$ tais que $L=\left(L_{1} \cap L_{2}\right) h$.

Outras representações envolvendo linguagens regulares são dadas a seguir.

$\underline{\text { TEOREMA } 9}-$ Salomaa

Seja $\Sigma$ um alfabeto, com $|\Sigma|=n$. Existem alfabetos $\Gamma_{1}$ e $\Gamma_{2}$, $\operatorname{com}\left|\Gamma_{1}\right|=n+2$ e $\left|\Gamma_{2}\right|=2 n+8$, morfismos $h_{1}: \Gamma_{1}^{*} \rightarrow \Sigma^{*}$ e $h_{2}: \Gamma_{2}^{*} \rightarrow \Gamma_{1}^{*}$ e uma linguagem de Dyck $D \subseteq \Gamma_{2}^{*}$ satisfazendo a seguinte condi ção:

para cada linguagem recursivamente enumerável $L \subseteq \Sigma^{*}$, existem linguagens regulares $R_{1}$ e $R_{2} \subseteq \Gamma_{2}^{*}$ tais que

$L=\left(\left(D \cap R_{1}\right) h_{2} \cap\left(D \cap R_{2}\right) h_{2}\right) h_{1}$.

Esse resultado segue dos Teoremas 6 e 7 .

o próximo Teorema é uma versão mais forte do ante- 
rior, onde a 1 inguagem L depende apenas de uma linguagem regular R.

TEOREMA 10 - Sa1oma a

Seja um alfabeto $\sum$ e $\Gamma=\bar{\Sigma} U\{a, b\}$. Existem 1 inguagens 1 ivres de contexto $\mathrm{L}_{1}$ e $\mathrm{L}_{2} \subseteq \Gamma^{*}$ e um morfismo $\mathrm{h}: \Gamma^{*} \longrightarrow \Sigma^{*} \operatorname{com}$ a seguinte propriedade:

para cada 1 inguagem recursivamente enumerāvel $L \subseteq \Sigma^{*}$, existe uma linguagem regular $R \subseteq \Gamma^{*}$ tal que $L=\left(L_{1} \cap L_{2} \cap R\right) h$.

Recentemente, depois dos resultados de Salomaa (1978) e de Culik (1979b) que estabeleceram representações de 1 inguagens recursivamente enumeräveis como imagens homomórficas (ou por dgsm) de conjuntos-igualdade de morfismos de monóides livres, värias outras representações têm sido apresentadas por Culik (1979a), Culik e Maurer (1979) e Engelfriet e Rozenberg $(1980)$.

A seguir, expomos os resultados mais significativos, dando antes algumas definições.

Seja f uma função sobre $\Sigma^{*}$. Então,o conjunto-ponto- $f i x_{0}$ de $f$, denotado por $F(f)$, é constituído pelas palavras que são iguais à sua imagem por f. Ou seja,

$F p(f)=\left\{x \in \Sigma^{*} / x f=x\right\}$.

Dado um alfabeto $\Sigma, L_{\Sigma}$ denota o conjunto de todas 
as palavras wem $(\Sigma \cup \bar{\Sigma}) *$ tais que $\left(\overline{w^{2} h_{\Sigma}}\right)=w h_{\bar{\Sigma}}$, onde $h_{\Sigma}\left(h_{\bar{\Sigma}}\right)$ é o morfismo que preserva $\Sigma(\bar{\Sigma})$.

$$
\text { Ou seja, } L_{\Sigma}=\underset{x \in \Sigma^{*}}{U} x w \bar{x} \text {, onde a operação } w \bar{e} \text { defini }
$$
da da seguinte forma. Sejam s e $t$ em $\Sigma^{*}$, então

$$
s w t=\left\{s_{1} t_{1} s_{2} t_{2} \ldots s_{n} t_{n} / s_{i}, t_{i} \in \Sigma^{*}(1 \leq i \leq n), s_{1} s_{2} \ldots s_{n}=s \quad \text { e } t_{1} t_{2} \ldots t_{n}=t\right\} .
$$

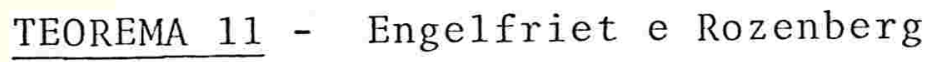

Para cada 1 inguagem recursivamente enumerável $L \subseteq \Sigma^{*}$, existe uma função dgsm g tal que $L=(F p(g)) h_{\Sigma}$, onde $h_{\Sigma}$ é o morfismo que preserva $\Sigma$.

TEOREMA 12 - Engelfriet e Rozenberg

Para cada linguagem recursivamente enumerável L, existem mor fismos $h_{0}, h_{1}$ e $h_{2}$ e uma linguagem regular $R$ tais que $L=\left(E q\left(h_{1}, h_{2}\right) \cap R\right) h_{0}$.

$\underline{\text { TEOREMA } 13}$ - Engelfriet e Rozenberg

Para cada 1 inguagem recursivámente enumerável L, existem uma substituição finita $f$, um morfismo $h$ e uma linguagem regular $R$ tais que $L=(F p(f) \cap R) h$. 
TEOREMA 14 - Engelfrict c Rozenberg

Para cada 1inguagem recursivamente enumerável L, existcm um a 1 fabeto $\Delta$, uma linguagem 1inear determinística $M \subseteq \Delta^{+} \Delta^{+}$e um morfismo tais que.

$$
L=\left(\left\{w(\bar{w} \rho) / w \in \Delta^{+}\right\} \cap M\right) h .
$$

A função reversão $\rho: \Delta^{*} \longrightarrow \Delta^{*}$ è definida por

$$
\begin{aligned}
& 1 \rho=1 \\
& \forall x \in \Delta^{+}, x=x_{1} \ldots x_{n}, \text { com } x_{i} \in \Delta(1 \leq i \leq n), \\
& x \rho=\left(x_{1} \ldots x_{n}\right) \rho=x_{n} \ldots x_{1} .
\end{aligned}
$$$$
\text { e }
$$

TEOREMA 15 - Engelfriet e Rozenberg

Para cada linguagem recursivamente enumerável L, tem-se que:

(i) existem um alfabeto $\Sigma$, um morfismo h e uma linguagem regular $\mathrm{R}$ tal que $\mathrm{L}=\left(\mathrm{L}_{\Sigma} \cap \mathrm{R}\right) \mathrm{h}$;

(ii) existem morfismos $f$ e $h$ e uma linguagem regular $\mathrm{R}$ tais que $L=\left(L_{\{a, b\}} f^{-1} \cap R\right) h$;

(iii) existe uma função dgsm g ta1 que $L=L_{\{a, b\}}$.

E interessante notar que a representação dada em (i) è anảloga ao Teorema de Chomsky e Schutzenberger para 1in- 
guagens livres de contexto.

TEOREMA 16 - Culik e Maurer

Existe uma linguagem recursivamente enumerável $U \subseteq\{a, b\}$ * tal que para cada linguagem recursivamente enumeráve $1 \mathrm{~L} \subseteq \Sigma^{*}$ exi $\underline{s}$ tem um morfismo h e um morfismo $f_{\Sigma}$, que preserva $\Sigma$, tais que $L=\left(U h^{-1}\right) f_{\Sigma}$.

TEOREMA 17 - Cu1ik e Maurer

Existe uma linguagem recursivamente enumerável $U \subseteq\{a, b\}$ * tal que toda linguagem recursivamente enumerável L pode ser escrita como $\mathrm{L}=\mathrm{Uh}^{-1}$, para algum morfismo $\mathrm{h}$. 


\section{CAPITULO 5}

\section{A REGULARIDADE DE CONJUNTOS-IGUALDADE DE MORFISMOS}

0 fato de conjuntos-igualdade de morfismos elementa res serem regulares foi de importância fundamental na solução apresentada por Ehrenfeucht e Rozenberg (1978) da decidibilidade do Problema da Equivalência da Sequência para os Sistemas DOL. E isto motivou a investigação de outras restrições sobre os morfismos para que seus conjuntos-igualdade fossem regulares, o que contribuiu para a solução de novos problemas na Teoria de Sistemas de Lindenmayer, como se pode ver em Enge1friet e Rozenberg (1979) e Rozenberg e Salomaa (1980).

Assim, estudamos inicialmente alguns resultados conhecidos em que o conjunto-igualdade $\mathrm{Eq}(f, g)$ è regular.

Em seguida, como consequência da indecidibilidade do 
Problema de Correspondência de Post para códigos de atraso um, concluímos que alguns dos conjuntos-igualdade regulares não são efetivamente regulares.

Além disso, com relação à representação homomórfica de 1 inguagens recursivamente enumerāveis, que obtivemos no Ca pítulo 4, concluímos que não se pode considerar os morfismos $\mathrm{h}_{1}$ e $\mathrm{h}_{2}$ como sendo ambos códigos de atraso limitado no mesmo sentido ou um deles sendo cödigo de atraso limitado, porque nesses casos $E q\left(h_{1}, h_{2}\right)$ é regular.

\section{RESTRICOEES SOBRE OS MORFISMOS PARA QUE SEUS CONJUNTOS- -IGUALDADE SEJAM REGULARES}

Um tópico importante no estudo dos conjuntos-igualdade de morfismos de monóides livres é encontrar restrições so bre os morfismos $f$ e $g$ de modo que o conjunto-igualdade Eq(f,g) seja regular.

Apresentamos, a seguir, alguns resultados conhecidos nesse sentido e que podem ser encontrados em Courcelle (1978) ou Karhumaki e Simon (1979) ou Rozenberg e Salomaa (1980).

TEOREMA 1 - Sejam $f$ um morfismo periódico e $g$ um morfismo injetor. Então, existe efetivamente uma palavra w (possivelmente vazia) tal que $\mathrm{Eq}(f, g)=w^{*}$.

Lembremos que um morfismo $h: \Gamma^{*} \longrightarrow \Delta^{*}$ é periōdico se existir 
algum $z$ em $\Delta^{*}$ tal que para cada $\gamma$ em $\Gamma, \gamma h$ pertence a $z^{*}$.

TEOREMA 2 - Sejam $f$ e g cỏdigos de atraso limitado no mesmo sen tido. Então, o conjunto-iguaIdade Eq $(f, g)$ è regular.

TEOREMA 3 - Sejam $f$ e g morfismos. Se $f$ è um cỏdigo de atraso limitado então o conjunto-igualdade Eq(f,g) è regular.

o próximo resultado segue do Teorema 2 ou do Teorema 3 .

COROLARIO 1 - Sejam $f$ e g códigos de atraso limitado. Então, o conjunto-igualdade $\mathrm{Eq}_{\mathrm{q}}(\mathrm{f}, \mathrm{g})$ è regular.

\section{늠}

Com a finalidade de demonstrarmos os Teoremas 2 e 3, provamos inicialmente dois lemas.

LEMA 1 - Para cada $k \geq 0$ e morfismos $f$ e $g$, o conjunto $E q_{k}(f, g)=\{w / w f=w g$ e para cada segmento inicial próprio u de $w$, ||$u f|-| u g|| \leq k\}$

é regular e pode ser construído efetivamente.

DEMONSTRAÇAO - Vamos supor que $\mathrm{Eq}_{\mathrm{k}}(\mathrm{f}, \mathrm{g}) \subseteq \Sigma^{*}$.

A afirmação ê verdadeira para $k=0$ porque, neste caso, $\mathrm{Eq}_{\mathrm{k}}(f, g)$ é $\{1\}$ ou $\Sigma_{1}^{*}$, para algum subconjunto $\Sigma_{1}$ de $\Sigma$.

Suponhamos então que $k \geq 1$ e vamos construir um autômato finito deterministico A como segue, utilizando o formalis mo de Simon (1981). 
O nümero de fitas de $A$ è um; os alfabetos de trabaTho e de entrada são iguais a $\Sigma$; o conjunto de estados de $A$ é dado por

$$
Q=\{p, r\} \cup Q_{1},
$$

onde $\quad Q_{1}=\left\{x_{+}, x_{-} / x \in \Sigma^{+}\right.$e $\left.|x| \leq k\right\}$

e $\mathrm{p}$ é o estado inicial e final de $\mathrm{A}$; o conjunto E das arestas de $A$ é a união dos conjuntos $E_{1}$ e $E_{2}$ que descrevemos a seguir. Para cada $\sigma$ em $\Sigma$ e para cada y em $\Sigma^{+}$, com $|y| \leq k$,

$$
\begin{aligned}
& \text { se } \sigma f=\sigma g \text { então }\left(p, L_{\sigma}, p\right) \in E_{1} ; \\
& \text { se } \sigma f=(\sigma g) x \text { e }|x| \leq k \text { então }\left(p, L_{\sigma}, x_{+}\right) \in E_{1} ; \\
& \text { se } \sigma g=(\sigma f) x \text { e }|x| \leq k \text { então }\left(p, L_{\sigma}, x_{-}\right) \in E_{1} ; \\
& \text { se } \sigma g=y(\sigma f) \text { então }\left(y_{+}, L_{\sigma}, p\right) \in E_{1} ; \\
& \text { se } y(\sigma f)=(\sigma g) z \text { e }|z| \leq k \text { então }\left(y_{+}, L_{\sigma}, z_{+}\right) \in E_{1} ; \\
& \text { se } \sigma g=y(\sigma f) z \text { e }|z| \leq k \text { então }\left(y_{+}, L_{\sigma}, z\right) \in E_{1} ; \\
& \text { se } \sigma f=y(\sigma g) \text { então }\left(y_{-}, L_{\sigma}, p\right) \in E_{1} ; \\
& \text { se } \sigma f=y(\sigma g) z \text { e }|z| \leq k \text { então }\left(y_{-}, L_{\sigma}, z_{+}\right) \in E_{1} ; \\
& \text { se } y(\sigma g)=(\sigma f) z \text { e }|z| \leq k \text { então }\left(y_{-}, L_{\sigma}, z z_{-}\right) \in E_{1} .
\end{aligned}
$$$$
0 \text { conjunto } \mathrm{E}_{2} \text { é constituído pelas arestas }\left(\mathrm{q}, \mathrm{L}_{\sigma}, \mathrm{r}\right)
$$
tais que não existem em $E_{1}$ arestas em que a primeira componen te é q e a segunda $\vec{e} L_{\sigma}$, para algum $\sigma$ em $\Sigma$. 
E fäcil verificar que o autônato A reconhece $\mathrm{Eq}_{\mathrm{k}}(\mathrm{f}, \mathrm{g})$, ou seja, que $w \in E_{q_{k}}(f, g)$ sse existe um passeio $c: p \longrightarrow p \mathrm{~cm}$ A tal que $w|c|=1$. Porque, sempre que $A$ estā num estado $x_{+} \in Q_{1}$ $\left(x_{-}\right)$, significa que a palavra w lida até aquele instante satisfaz $w f=(w g) x(w g=(w f) x)$ e quando $A$ se encontra no estado p significa que a palavra w lida até aquele instante satisfaz $w f=w g$.

LEMA 2 - Sejam $\mathrm{h}_{1}$ e $\mathrm{h}_{2}: \mathrm{L}^{*} \longrightarrow \Delta^{*}$ dois códigos de atrasos $\mathrm{k}_{1}$ e $k_{2}$, respectivamente, da esquerda para a direita. Sejam $k=\max \left\{k_{1}, k_{2}\right\}$ e $m=\max \left\{\left|\sigma h_{i}\right| / \sigma \in \Sigma, i=1,2\right\}$. Seja $z$ em $\Delta^{*}$, com $|z| \geq(k+1) m$ tal que $\left(u_{0} h_{1}\right) z=u_{0} h_{2}$, para algum segmento inicial $u_{0}$ de uma palavra em $E_{q}\left(h_{1}, h_{2}\right)$. Então, existe uma palavra w em $\Sigma^{*}$ tal que $\left(w h_{1}\right) y=z\left(w h_{2}\right)$, com $|y|<(k+1) m$ e para cada $u \in \Sigma *$ tal que $\left(u h_{1}\right) z=u h_{2}$, se $u v \in E q\left(h_{1}, h_{2}\right)$ então w é um segmento $\underline{i}$ nicial de $v$.

DEMONSTRACÃO - Seja u em $\Sigma^{*}$ tal que $\left(u_{1}\right) z=u h_{2}$.

Podemos observar que uv $\in \mathrm{Eq}\left(\mathrm{h}_{1}, \mathrm{~h}_{2}\right)$ sse $\mathrm{vh}_{1}=\mathrm{z}\left(\mathrm{vh}_{2}\right)$.

Afirmamos que existe no máximo um $\sigma$ em $\Sigma$ tal que uo è um segmento inicial de uv $\in \mathrm{Eq}\left(\mathrm{h}_{1}, \mathrm{~h}_{2}\right)$.

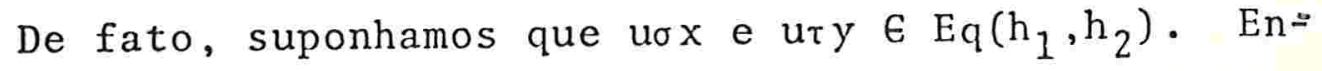
tão, $u h_{2}=\left(u h_{1}\right) z$ è um segmento inicial de $(u \sigma x) h_{1}$ e $\left(u h_{1}\right) z$ tam bém é um segmento inicial de (uty)h $h_{1}$.

Logo, z è um segmento inicial de ambos $(\sigma x) h_{1}$ 
$(\tau y) h_{1}$. Como $|z| \geq(k+1) m$ e uox $\in \mathrm{Eq}\left(\mathrm{h}_{1}, \mathrm{~h}_{2}\right)$ segue que existe um segmento inicial $t$ de $x$ tal que $(\sigma t) h_{1}$ é um segmento inicial. de $z$. e $\left|(\sigma t) h_{1}\right|>m k$.

Por outro 1ado, $(\sigma t) h_{1} \bar{e}$ um segmento inicial de $(\tau y) h_{1}$. Mas, $h_{1}$ é um código de atraso $k_{1}$ da esquerda para a direita; portanto, resulta que $\sigma=\tau$.

Um tal $\sigma$ em $\Sigma$ existe porque $u_{0} \bar{e}$ um segmento inicial de $u_{o} v_{0}$ em $E q\left(h_{1}, h_{2}\right)$ e $\sigma$ não depende de $u$.

Se ||$(u \sigma) h_{1}|-|(u \sigma) h_{2}||<(k+1) m$, podemos considerar $w=\sigma e$, nesse caso, existe uma palavra $y$ em $\Delta^{+}$tal que

$$
(u \sigma) h_{1} y=(u \sigma) h_{2}=\left(u h_{2}\right)\left(\sigma h_{2}\right)=\left(u h_{1}\right) z\left(\sigma h_{2}\right) \text {. }
$$

Logo,

$$
\left(\sigma h_{1}\right) y=z\left(\sigma h_{2}\right) \text { e }|y|<(k+1) m \text {. }
$$

Caso contrârio, consideramos $\sigma$ como a primeira letra de w e determinamos a seguinte de modo análogo com uo pa ra $u$ e $z_{1}$, tal que $(u \sigma) h_{1} z_{1}=(u \sigma) h_{2}$, para $z$.

0 fato de $u_{0}$ ser um segmento inicial de uma palavra $\mathrm{u}_{\mathrm{o}} \mathrm{v}_{\mathrm{o}}$ em $\mathrm{Eq}\left(\mathrm{h}_{1}, \mathrm{~h}_{2}\right)$ garante a existência da palavra w que satis faça as condições do enunciado.

Vamos então demonstrar o Teorema 2 que enunciamos no vamente.

$\underline{\text { TEOREMA } 2}$ - Sejam f e g códigos de atraso limitado no mesmo 
sentido. Então, o conjunto-igualdade $\mathrm{E}_{\mathrm{q}}(\mathrm{f}, \mathrm{g})$ é regular.

DEMONSTRACKO - Suponhamos que $\mathrm{f}$ e $\mathrm{g}: \Sigma^{*} \rightarrow \Delta^{*}$ sejam códigos de atrasos $k_{1}$ e $k_{2}$, respectivamente, da esquerda para a direita. Sejam $k=\max \left\{k_{1}, k_{2}\right\}$ e $m=\max \{|\sigma f|,|\sigma g| / \sigma \in \Sigma\}$.

Para mostrarmos que $\mathrm{E}_{\mathrm{q}}(f, g)$ è regular basta provarmos que existe um $n \geq 0$ tal que para cada $u$ em $\Sigma^{+}$que seja seg mento inicial de alguma palavra em Eq(f,g), ||$u f|-| u g|| \leq n$.

Para cada palavra $u$ em $\Sigma^{*}$, dizemos que $u \beta=|| u f|-| u g||$ è o bazanco de $\mathrm{u}$.

Seja $v \in E_{q}(f, g)$ e $u$ um segmento inicial de $v$ tal que

$$
\text { (uf) } x=u g \quad \text { e } \quad|x| \geq(k+1) m \text {. }
$$

Então, existem duas palavras mais curtas $v_{1}$ e $v_{3}$ tais que

$$
\begin{aligned}
& \mathrm{u}=\mathrm{v}_{1} \mathrm{v}_{2}, \\
& \mathrm{v}=\mathrm{v}_{1} \mathrm{v}_{2} \mathrm{v}_{3} \mathrm{v}_{4}, \\
& \left(\mathrm{v}_{1} \mathrm{~h}_{1}\right) \mathrm{z}=\mathrm{vh}_{2}, \text { com }(\mathrm{k}+1) \mathrm{m} \leq|\mathrm{z}| \leq(\mathrm{k}+2) \mathrm{m} \\
& \left(\mathrm{v}_{1} \mathrm{v}_{2} \mathrm{v}_{3}\right) \mathrm{h}_{1} \mathrm{y}=\left(\mathrm{v}_{1} \mathrm{v}_{2} \mathrm{v}_{3}\right) \mathrm{h}_{2}, \text { para algum y ta1 que } \\
& \text { e } \quad|\mathrm{y}|<(\mathrm{k}+1) \mathrm{m} .
\end{aligned}
$$

Logo, pelo Lema 2 resulta que a palavra w associada a $z$ é $w=v_{2} v_{3}$ e para determinä-la temos calculado todos os $\left(v_{1} s\right)$ B tais que $v_{1} s$ é um segmento inicial de $v_{1} v_{2} v_{3}$. Então, 
temos computado também $\left(v_{1} v_{2}\right) \beta=u \beta$.

Mas, existe um nümero finito de palavras $z$ nas condições acima e portanto, tambẻm das correspondentes palavras w. Logo, existe um número finito de valores para os balanços das palavras $s$ que são segmentos iniciais de palavras em Eq(f,g) tal que sf é um segmento inicial de sg e, portanto, podemos de terminar o seu máximo. Vamos denotá-lo por $n_{1}$. Desse modo, $|x| \leq n_{1}$

Analogamente, se a palavra u é tal que

$$
(u f)=(u g) x \text { e }|x| \geq(k+1) m,
$$

existe uma constante $n_{2}$ tal que $|x| \leq n_{2}$.

Assim, sendo $n=\max \left\{n_{1}, n_{2}\right\}$ temos que

$\max \left\{u_{\beta} / \mathrm{u}\right.$ é um segmento inicial de $\left.v \in E q(f, g)\right\}=n$.

Portanto, como $\mathrm{Eq}(f, g)=\mathrm{Eq}_{\mathrm{n}}(f, g)$, segue pelo Lema 1 que Eq $(f, g)$ è regular.

OBSERVAÇAO - Se $f$ e g são códigos de atraso limitado da dire ta para a esquerda, a demonstração é anāloga, utilizando o dual do Lema 2.

A seguir, demonstramos o Teorema 3 cujo enunciado re produzimos a seguir. 
TEOREM 3 - Sejam f e g morfismos. Se f é um código de atraso limitado então o conjunto-igualdade Eq(f,g) é regular.

DEMONSTRACKO - Sejam os morfismos $f$ e $g: \Sigma^{*} \longrightarrow \Delta^{*}$.

Suponhamós que $f$ é um código de atraso $k$ e se ja $m=\max \{|\sigma f|,|\sigma g| / \sigma \in \Sigma\}$.

Como na demonstração do Teorema 2 é suficiente mostrarmos que, para cada $u$ em $\Sigma^{+}$que ê um segmento inicial de alguma palavra em Eq $(f, g)$ e

$$
u g=(u f) x \text { ou } u f=(u g) x, \quad \text { com }|x| \geq(k+1) m,
$$

existe um ünico o $€ \Sigma$ tal que uoy $€ E q(f, g)$.

Se $u g=(u f) x$ e $|x| \geq(k+1) m$, segue que existe um único $\sigma$ nas condições acima, pois $f$ è de atraso limitado.

Consideremos então uma palavra u tal que u seja um segmento inicial de uv em Eq(f,g) e $u f=(u g) x$, com $|x| \geq(k+1) m$.

Seja $u \sigma y$ em Eq $(f, g)$.

Como $\mid$ uf $|-| u g \mid \geq(k+1) m$ segue que $|y g|-|y f| \geq(k+1) m$, pois $(u \sigma y) g=(u \sigma y) f=(u g) \times(\sigma y) f ;$ o que implica que $(\sigma g)(y g)=$ $=x(\sigma f)(y f)$.

Mas, yf é um segmento final de yg e como $f$ é de atra so limitado, segue que tal o é único. 
2. A NXO EFETIVIDADE DE $\Lambda$ LGUNS CONJUNTOS-IGUALDADE REGULARES

Na seção anterior verificamos a existência de alguns conjuntos-igualdade de morfismos de monóides livres que são regulares.

Agora, vamos estudar a efetividade desses conjuntos. Isto $\bar{e}$, dizemos que um conjunto L é efetivamente reguzar se é possivel construir algoritmicamente um autômato finito que re conheça L.

Assim, como consequência da indecidibilidade do Pro blema de Correspondência de Post para códigos de atraso um, ob temos os resultados que apresentamos a seguir.

TEOREMA 4 - Sejam $f$ e g códigos de atraso um. Então, o conjunto-igualdade $\mathrm{Eq}_{\mathrm{q}}(\mathrm{f}, \mathrm{g})$ è regular, mas não é efetivamente regular. Em particular, não è decidível se $E_{q}(f, g) \backslash\{1\}=\emptyset$.

DEMONSTRAÇAO - Sejam $f$ e g códigos de atraso um.

Vamos considerar uma instância do Problema de Corres pondência de Post com os códigos $f$ e $g$. Então, o seu conjunto de soluções $\vec{e} \operatorname{Eq}(f, g) \backslash\{1\}$.

Por outro lado, segue pelo Corolärio 1 que Eq(f,g)é regular.

Suponhamos, por absurdo, que $\mathrm{E}_{\mathrm{q}}(f, g)$ possa ser cons

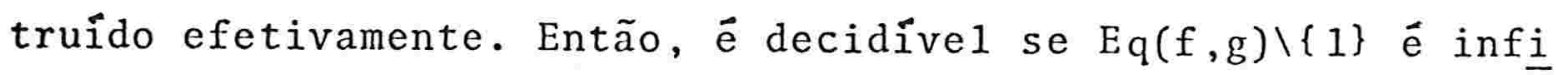


nito ou não (ou se $E_{q}(f, g) \backslash\{1\}$ e vazio ou não).

Assim, ē possível decidir se o Problema de Correspon dência de post com os côdigos $f$ e g tem solução ou não; o que contradiz o Teorema 2 (Capítulo 3), pois os morfismos $f$ e $g$ são côdigos de atraso um.

Portanto, $\mathrm{E}_{\mathrm{q}}(f, g)$ não é efetivamente regular.

COROLARRIO 2 - Sejam f e g cödigos de atraso um no mesmo senti do. Então, o conjunto-igualdade Eq(f,g) não è efetivamente re gular.

DEMONSTRACÃO - E uma consequência imediata dos Teoremas 2 e 4.

COROLÁRIO 3 - Sejam $f$ e g morfismos. Se f é um cödigo de atra so um então o conjunto-igualdade Eq(f,g) não ê efetivamente re gular.

DEMONSTRAÇÃO - Segue imediatamente dos Teoremas 3 e 4.

3. CONJUNTOS-IGUALDADE NAO REGULARES

Analisamos agora os conjuntos-igualdade de morfismos que são códigos de atraso limitado em sentidos opostos.

Vamos considerar o seguinte exemplo de Karhumáki e Simon (1979) . 
$\underline{\text { EXEMPLO }}-$ Sejam $f$ e $g:\{a, b, c, d, e, f\} * \longrightarrow\{0,2,3,4,5\} *$ morfis mos definidos pela tabela abaixo:

\begin{tabular}{|c|llllll|}
\hline$\sigma$ & $\mathrm{a}$ & $\mathrm{b}$ & $\mathrm{c}$ & $\mathrm{d}$ & $\mathrm{e}$ & $\mathrm{f}$ \\
\hline$\sigma f$ & 0234 & 2323 & 4 & 24 & 32 & 5 \\
\hline$\sigma g$ & 0 & 23 & 4 & 42 & 3232 & 4325 \\
\hline
\end{tabular}

A palavra $w=a b c b^{2} d e^{2} \operatorname{cef}$ estä em $E_{q}(f, g)$ pois

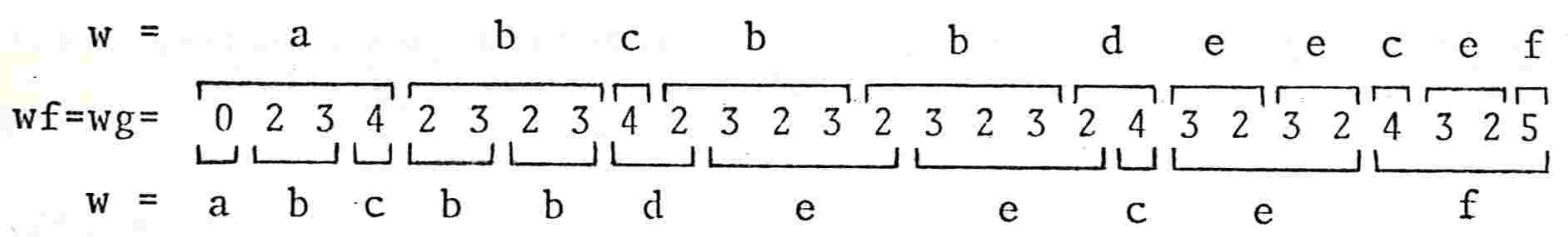

Pode-se verificar que $f$ é um prefixo; então $f$ é um código de atraso zero da esquerda para a direita. Porém, f não é de atraso limitado da direita para a esquerda.

Tambëm, pode-se verificar que g è um sufixo; logo,g è um código de atraso zero da direita para a esquerda. Mas, g não é de atraso limitado da esquerda para a direita.

Além disso, pode-se.ver que

$$
E q(f, g)=\left(\left\{a b c b^{2} c b^{4} c \ldots c b^{2^{n}} d e^{2^{n}} c \ldots c e^{2} \operatorname{cef} / n \geq 0\right\} \quad U\{c\}\right) *
$$

Portanto, Eq(f,g) não ë nem livre de contexto. 
Assim, concluímos que existem morfismos $\mathrm{f} c \mathrm{~g}$, sendo que $f$ è um cỏdigo de atraso zero da esquerda para a direita e g da direita para a esquerda, de modo que o conjunto $E_{q}(f, g)$ não ê regular.

Por outro lado, a existência de códigos $f$ e $g$ de atraso um em sentidos opostos, tal que Eq(f,g) não é regular, segue imediatamente do Teorema 3 (Capítulo 4) e do fato de que a imagem homomórfica de um conjunto regular ê regular.

4. RESTRICOOES SOBRE OS MORFISMOS QUE REPRESENTAM AS LINGUAGENS RECURSIVAMENTE ENUMERÃVEIS ATRAVES DE CONJUNTOS-IGUALDADE

Vamos considerar a representação de linguagens rear sivamente enumeräveis dada pelo Teorema 3 do Capítulo 4; ou seja, se L é uma linguagem recursivamente enumerảvel então $L=\left(\right.$ eq $\left.\left(h_{1}, h_{2}\right)\right) h_{0}$, onde $h_{0}, h_{1}$ e $h_{2}$ são morfismos tais que $h_{1}$ é um código de atraso um da esquerda para a direita e $h_{2}$ da direita para a esquerda.

Essa representação descreve cada linguagem recursivamente enumerāvel como a imagem homomórfica de um conjunto-i gualdade minimal pertencente a uma classe mais restrita de conjuntos do que a considerada por Culik no Teorema 2 (Capítu 104 ) porque, pelo Corolärio 1 (Capitulo 1 ), eq(IHOM) $\underset{\neq}{\subset} \mathrm{eq}(\mathrm{HOM})$. Além disso, com relação aos morfismos $h_{1}$ e $h_{2}$, pode 
mos concluir que:

(19) eles não podem ser simultaneamente cödigos de atraso li mitado no mesmo sentido;

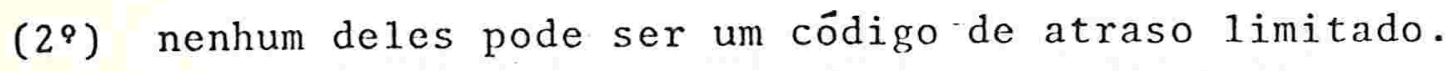

De fato, porque em cada um desses casos, respectiva mente, pelos Teoremas 2 e 3 , o conjunto-igualdade $E_{q}\left(h_{1}, h_{2}\right)$ se ria regular e, consequentemente eq $\left(h_{1}, h_{2}\right)$ também seria regular. Então, como a família das linguagens regulares é fechada sob morfismo, (eq $\left.\left(h_{1}, h_{2}\right)\right) h_{o}=L$ seria regular; o que contradiz o Teorema 3 (Capitulo 4). 


\section{REFERENCIAS BIBLIOGRAFICAS}

CHOMSKY, N. \& SCHUTZENBERGER, M.P. 1963. The algebraic theo ry of context-free languages. In: BRAFFOR', P. \& HIRSCHBERG, D., eds. Computer programming and formal systems. Amsterdam, North-Holland. p.118-161. (Studies in Logic and the Foundations of Mathematics)

COURCELlE, B. 1978. Problemes d'equivalence pour les systemes de Lindenmayer de type DOL. In: SEMINAIRE D'INFORMATIQUE THEORIQUE, 1977-78, S.L.p., s.c.p. p.1-16.

CULIK, K. 1979a. On the homomorphic characterizations of $f \underline{a}$ milies of languages. In: MAURER, H.A., ed. Automata, languages and programming. Berlin, Springer. p.161-170. (Lec ture Notes in Computer Science, 71)

CULIK, K. 1979b. A purely homomorphic characterization of recursively enumerable sets. Journal of the Association for Computing Machinery, 26(2):345-350.

CULIK, K. \& FRIS, I. 1977. The decidability of the equivalence problem for DOL-systems. Information and Control, $35(1): 20-39$.

CULIK, K. \& MAURER, H.A. 1979. On simple representations of language families. R.A.I.R.O. Informatique Thēorique, $13(3): 241-250$. 
EHRENFEUCIT, $\Lambda$. \& ROZENBERG, G. 1978. Elementary homomorphisms and a solution of the DOL sequence equivalence prob1em. Theoretical Computer Science, 7(2):169-183.

EHRENFEUCHT, A.; KARHUMÄKI, J.; ROZENBERG, G. 1982. The (generalized) Post correspondence problem with lists consisting of two words is decidable. Theoretical computer Science, $21(2): 119-144$.

EILENBERG, S. 1974. Automata, languages, and machines. New York, Academic Press. v.l. (Pure and Applied Mathematics, 59)

ENGELFRIET, J. \& ROZENBERG, G. 1979. Equality languages and fixed point languages. Information and Control, 43(1):2049 .

ENGELFRIET, J. \& ROZENBERG, G. 1980. Fixed point languages, equality languages, and representation of recursively enumerable languages. Journal of the Association for Computing Machinery, 27(3):499-518.

HOPCROFT, J.E. \& ULLMAN, J.D. 1969. Formal languages and their relation to automata. Reading, Addison-Wesley. 242p.

KARHUMÄKI, J. \& SIMON, I. 1979. A note on elementary homo morphisms and the regularity of equality sets. Bulletin of the European Association for Theoretical Computer Science, $9: 16-24$.

LECERF, M.Y. 1963a. Machines de Turing réversibles. Rëcursive insolubilité en $n \in N$ de l'equation $u=\theta^{n} u$, où $\theta$ est un <isomorphisme de codes > Comptes Rendus de l'Académie des Sciences, 257:2597-2600. 
LECERF, M.Y. 1963b. Récursive insolubilitê de l'equation gênërale de diagonolisation de deux monomorphismes de monoides livres $\phi x=\psi x$. Comptes Rendus de l'Académie des Sciences, 257:2940-2943.

LUCCHESI, C.L. et a1ii. 1979. Aspectos teōricos da compu tação. Rio de Janeiro, IMPA. 292p. (Projeto Euclides)

PERRIN, D., ed. 1980. Theorie des codes. In: ECOLE DF PRIN TEMPS D'INFORMATIQUE THEORIQUE, $7^{\mathrm{a}} \cdot$, Paris, 1979 . Actes. Paris, Laboratoire D'Informatique Thêorique et Programmation. 280p.

POST, E.L. 1946. A variant of a recursively unsolvable pro blem. Bulletin of the American Mathematical Society, 52: 264-268.

ROZENBERG, G. \& SALOMAA, A. 1980. The mathematical theory of L systems. New York, Academic Press. 352p.

SALOMAA, A. 1973. Formal languages. New York, Academic Press. 322p. (ACM Monograph Series)

SALOMAA, A. 1978. Equality sets for homomorphisms of free monoids. Acta Cybernetica, 4:127-139.

SIMON, I. 1981. Linguagens formais e autômatos. Campinas, IMECC-UNICAMP. 200p. Trab. apres. à 2a Escola de Computa ção, Campinas, 1981. 


\section{INDICE DE SIIMBOLOS}

\begin{tabular}{|c|c|c|c|}
\hline $\begin{array}{l}\mathrm{Eq}(f, g) \cdots \ldots \ldots \\
\mathrm{eq}(f, g) \cdots \cdots\end{array}$ & $\begin{array}{l}7 \\
9\end{array}$ & $A \ldots \ldots \ldots \ldots \ldots \ldots \ldots$ & 18 \\
\hline$E q_{k}(f, g) \cdots \cdots \cdots$ & 165 & $Q_{A}, T_{A}, \Sigma_{A}, I_{A}, A_{A} \cdots \cdots \cdots$ & 18 \\
\hline $\mathrm{Eq}(\mathrm{HOM}) \quad \cdots \cdots \cdots$ & 9 & $E_{A}, D_{A} \cdots \cdots \cdots \cdots \cdots \cdots \cdots$ & 18 \\
\hline $\begin{array}{l}\mathrm{Eq}(\mathrm{IHOM}) \cdots \cdots \\
\mathrm{eq}(\mathrm{HOM}) \cdots \cdots\end{array}$ & $\begin{array}{l}10 \\
16\end{array}$ & $\mathrm{E}_{\sigma}, \mathrm{D}_{\sigma}, \mathrm{E}_{\sigma}^{-1}, \mathrm{D}_{\sigma}^{-1}, \mathrm{~V}, \mathrm{~N} \cdots \ldots$ & 18 \\
\hline eq $($ IHOM $) \ldots \ldots \ldots$ & 16 & $\begin{array}{l}\mathrm{A}_{A}(q) \ldots \ldots \ldots \ldots \ldots \ldots \\
\mathrm{CI}_{A} \cdots \cdots \cdots \cdots \cdots \cdots\end{array}$ & $\begin{array}{l}18 \\
23\end{array}$ \\
\hline $\operatorname{Fp}(f) \ldots \ldots \ldots$ & 159 & $|\alpha|,|c| \ldots \ldots \ldots \ldots \ldots \ldots \ldots \ldots$ & 22,25 \\
\hline$P, S, P S, S P \ldots \ldots \cdots$ & 5 & $\begin{array}{l}|| \alpha||,|| c|| \ldots \ldots \ldots \ldots \ldots \ldots \ldots \ldots \\
\Gamma_{\alpha} \mid \ldots \ldots \ldots \ldots \ldots \ldots \ldots\end{array}$ & $\begin{array}{r}23,25 \\
77\end{array}$ \\
\hline $\begin{array}{l}\underline{b} \cdots \cdots \cdots \cdots \\
\mathrm{h}_{\mathrm{e}}, \mathrm{h}_{\mathrm{d}} \cdots \cdots \cdots \cdots\end{array}$ & $\begin{array}{r}85 \\
124\end{array}$ & {$[\alpha],[c] \ldots \ldots \ldots \ldots \ldots \ldots \ldots \ldots$} & 80,81 \\
\hline$h_{\Sigma} \cdots \cdots \cdots \cdots \cdots$ & 6 & $\begin{array}{l}|A| \ldots \ldots \ldots \ldots \ldots \ldots \\
A^{\prime} \quad \ldots \ldots \ldots \ldots \ldots \ldots\end{array}$ & $\begin{array}{l}27 \\
29\end{array}$ \\
\hline $\begin{array}{l}L_{\Sigma} \ldots \ldots \ldots \ldots \ldots \ldots \\
L \ldots \ldots \ldots \ldots \ldots\end{array}$ & $\begin{array}{l}160 \\
160\end{array}$ & & \\
\hline
\end{tabular}




\section{INDICE DE DEFINICOEES}

acoplamento

a1fabeto

de entrada. . . . . . . . . . . . . . 18

de trabalho................. . 18

aresta . . . . . . . . . . . . . . 18

balanço.................... 169

codificação de uma computação. . . . . . . . . 80

código . . . . . . . . . . . . . . . . . . . . 3

de atraso limitado............... . . 4

de atraso $\mathrm{k}$ da direita para a esquerda. . . . . . . 3

de atraso $\mathrm{k}$ da esquerda para a direita. . . . . . 3

de atraso $k$ (em ambos os sentidos)......... 4

comprimento. ................. . . 24

computação . . . . . . . . . . . . . 24

configuração

das fitas de A. . . . . . . . . . . 20

instantânea de A. . . . . . . . . . 20

intermediāria de A. . . . . . . . . . 77

conjunto-

-igualdade de morfismos de monóides livres . . . . . 7

-igualdade minimal de morfismos de monóides livres. • 9

-ponto-fixo de uma função. . . . . . . . . . . 159 
efetivamente regular . . . . . . . . . . . . . .

função

codificação de uma computação associada a uma aresta

$$
\text { (passeio). } 80,81
$$

configuração instantânea após a execução de uma ares

$$
\text { ta (passeio). } \cdot 23,25
$$

configuração intermediāria associada a uma aresta . . 77 reversão . . . . . . . . . . . . . . 161

instrução . . . . . . . . . . . . . . . . . 18

linguagem reconhecida por A . . • . . . . . . . 27

māquina de Turing . . . . . . . . . . . . . . 18

deterministica. . . . . . . . . . . . . . 28

equivalente. . . . . . . . . . . . . . 27

normalizada. . . . . . . . . . . . . . 28

reversivel . . . . . . . . . . . . . . 30

semi-normalizada . . . . . . . . . . . 28

morfismo

biprefixo... . . . . . . . . . . . . . 5

elementar . . . . . . . . . . . . . 6

periōdico . . . . . . . . . . . . . . . 108

prefixo . . . . . . . . . . . . . . . . . . 4

que preserva um alfabeto. . . . . . . . . . . 6

sufixo . . . . . . . . . . . . . . . . . 4

origem . . . . . . . . . . . . . . . . 18,24

passeio. . . . . . . . . . . . . . . . . 24

Problema de Correspondência de Post. . . . . . . . 62 
reversa(o)

da aresta.. . . . . . . . . . . . . 30

da måquina de Turing. . . . . . . . . . . . . 29

do passeio. . . . . . . . . . . . . . . . . 30

rótulo •. . . . . . . . . . . . . . . . . 22, 25

tërmino. . . . . . . . . . . . . . . 18,24 